Supplementary Information

Synthesis, Antiviral Activity and Induction of Plant Resistance of Indole Analogues

Bearing Dithioacetal Moiety

Chunle Wei, Jian Zhang, Jing Shi, Xiuhai Gan, Deyu Hu,* Baoan Song *

State Key Laboratory Breeding Base of Green Pesticide and Agricultural Bioengineering, Key Laboratory of Green Pesticide and Agricultural Bioengineering, Ministry of Education, Guizhou University, Huaxi District, Guiyang 550025, China

*Corresponding author (Tel.: 86-851-88292170; Fax: 86-851-88292170; E-mail: dyhu@gzu.edu.cn; songbaoan22@yahoo.com) 
Table of Contents

1. Characterizations of products............................................... $3-18$

2. ${ }^{1} \mathrm{H},{ }^{13} \mathrm{C}$ NMR and HRMS data............................................ 


\section{Characterization of products}

\section{(1) Characterization of intermediates, C1-C4}

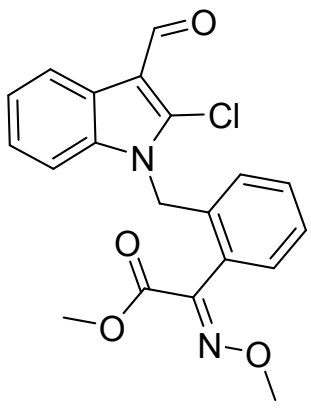

(E)-methyl-2-(2-((2-chloro-3-formyl-1H-indol-1-yl)methyl)phenyl)-

2-(methoxyimino)acetate (C1): Yield: 93 \%; yellow solid; m. p. 83.8 - $85.5{ }^{\circ} \mathrm{C} ;{ }^{1} \mathrm{H}$ NMR (400 MHz, DMSO- $\left.d_{6}\right) \delta 10.06(\mathrm{~s}, 1 \mathrm{H},-\mathrm{CH}=\mathrm{O})$, $8.14(\mathrm{~d}, J=4.4 \mathrm{~Hz}, 1 \mathrm{H}, \mathrm{Ar}-\mathrm{H}), 7.36$ (d, $J=7.5 \mathrm{~Hz}, 1 \mathrm{H}, \mathrm{Ar}-\mathrm{H}), 7.34$ 7.32 (m, 1H, Ar-H), 7.32 - 7.28 (m, 3H, Ar-H), 7.27 (d, J= $7.1 \mathrm{~Hz}, 1 \mathrm{H}$, Ar-H), $6.72(\mathrm{~d}, J=7.5 \mathrm{~Hz}, 1 \mathrm{H}, \mathrm{Ar}-\mathrm{H}), 5.35\left(\mathrm{~s}, 2 \mathrm{H},-\mathrm{NCH}_{2}-\right), 4.04(\mathrm{~s}$, $\left.3 \mathrm{H},=\mathrm{N}-\mathrm{OCH}_{3}\right), 3.74\left(\mathrm{~s}, 3 \mathrm{H},-\mathrm{CO}-\mathrm{OCH}_{3}\right) ;{ }^{13} \mathrm{C}$ NMR (100 MHz, DMSO- $\left.d_{6}\right) \delta 184.10(1 \mathrm{C})$, 162.87 (1C), 148.72 (1C), 137.20 (1C), 135.91 (1C), 134.18 (1C), 130.28 (1C), 129.57 (1C), 129.21 (1C), 128.15 (1C), 126.15 (1C), 124.62 (1C), 124.21 (1C), 123.95 (1C), 120.61 (1C), 112.78 (1C), 111.53 (1C), 64.05 (1C), 53.17 (1C), 45.56 (1C); HRMS (ESI) m/z for $\mathrm{C}_{20} \mathrm{H}_{18} \mathrm{O}_{4} \mathrm{~N}_{2} \mathrm{Cl}[\mathrm{M}+\mathrm{H}]^{+}$calcd 385.09496, found 424.09402.

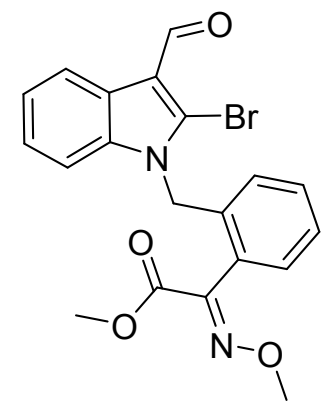

(E)-methyl-2-(2-((2-bromo-3-formyl-1H-indol-1-yl)methyl)phenyl)2-(methoxyimino)acetate (C2): Yield: 90 \%; yellow solid; m. p. 124.3 - $125.8{ }^{\circ} \mathrm{C} ;{ }^{1} \mathrm{H}$ NMR $\left(400 \mathrm{MHz}, \mathrm{DMSO}-d_{6}\right) \delta 9.99(\mathrm{~s}, 1 \mathrm{H},-\mathrm{CH}=\mathrm{O})$, $8.16(\mathrm{~d}, J=5.5 \mathrm{~Hz}, 1 \mathrm{H}, \mathrm{Ar}-\mathrm{H}), 7.35(\mathrm{t}, J=7.3 \mathrm{~Hz}, 1 \mathrm{H}, \mathrm{Ar}-\mathrm{H}), 7.29$ (d, $J=5.1 \mathrm{~Hz}, 2 \mathrm{H}, \mathrm{Ar}-\mathrm{H}), 7.26(\mathrm{t}, J=2.5 \mathrm{~Hz}, 3 \mathrm{H}, \mathrm{Ar}-\mathrm{H}), 6.58$ (d, $J=7.7$ $\mathrm{Hz}, 1 \mathrm{H}, \mathrm{Ar}-\mathrm{H}), 5.38\left(\mathrm{~s}, 2 \mathrm{H},-\mathrm{NCH}_{2}-\right), 4.06\left(\mathrm{~s}, 3 \mathrm{H},=\mathrm{N}-\mathrm{OCH}_{3}\right), 3.77(\mathrm{~s}$, $\left.3 \mathrm{H},-\mathrm{CO}-\mathrm{OCH}_{3}\right) ;{ }^{13} \mathrm{C}$ NMR (100 MHz, DMSO- $\left.d_{6}\right) \delta 185.37$ (1C), 162.93 (1C), 148.79 (1C), 137.23 (1C), 134.24 (1C), 130.25 (1C), 129.48 (1C), 129.16 (1C), 128.04(2C), 125.73 (1C), 125.20 (1C), 124.56 (1C), 123.76 (1C), 120.47 (1C), 115.28 (1C), 111.57 (1C), 64.08 (1C), 53.21 (1C), 46.84 (1C); HRMS (ESI) $\mathrm{m} / \mathrm{z}$ for $\mathrm{C}_{20} \mathrm{H}_{18} \mathrm{O}_{4} \mathrm{~N}_{2} \mathrm{Br}[\mathrm{M}+\mathrm{H}]^{+}$calcd 429.04445, found 429.04340 .<smiles>CO/N=C(/C(=O)OC)c1ccccc1Cn1c(Cl)c(C=O)c2cc(Br)ccc21</smiles>

(E)-methyl-2-(2-((5-bromo-2-chloro-3-formyl-1H-indol-1-yl)me thyl)phenyl)-2-(methoxyimino)acetate (C3): Yield: 85 \%; yellow solid; m. p. 109.3 - $112.1{ }^{\circ} \mathrm{C} ;{ }^{1} \mathrm{H}$ NMR (400 MHz, DMSO- $\left.d_{6}\right) \delta$ 10.03 (s, 1H, -CH=O), 8.26 (d, $J=2.0 \mathrm{~Hz}, 1 \mathrm{H}, \mathrm{Ar}-\mathrm{H}), 7.47$ (d, $J=$ $8.8 \mathrm{~Hz}, 1 \mathrm{H}, \mathrm{Ar}-\mathrm{H}), 7.41$ - 7.36 (m, 1H, Ar-H), 7.36 - 7.32 (m, 1H, 
Ar-H), 7.28 (d, $J=8.9 \mathrm{~Hz}, 1 \mathrm{H}, \mathrm{Ar}-\mathrm{H}), 7.26$ (d, $J=6.1 \mathrm{~Hz}, 1 \mathrm{H}, \mathrm{Ar}-\mathrm{H}), 6.77$ (d, $J=6.8 \mathrm{~Hz}$, $1 \mathrm{H}, \mathrm{Ar}-\mathrm{H}), 5.37$ (s, $\left.2 \mathrm{H},-\mathrm{NCH}_{2}-\right), 4.02\left(\mathrm{~s}, 3 \mathrm{H},=\mathrm{N}-\mathrm{OCH}_{3}\right), 3.72\left(\mathrm{~s}, 3 \mathrm{H},-\mathrm{CO}-\mathrm{OCH}_{3}\right) .{ }^{13} \mathrm{C} \mathrm{NMR}$ $\left(100 \mathrm{MHz}, \mathrm{DMSO}-d_{6}\right) \delta 184.15(1 \mathrm{C}), 162.78$ (1C), 148.66 (1C), 138.04 (1C), $134.78(1 \mathrm{C})$ 133.89 (1C), 130.30 (1C), 129.61 (1C), 129.28 (1C), 128.26 (1C), 127.22 (1C), 126.39 (1C), 125.81 (1C), 122.64 (1C), 116.72 (1C), 113.77 (1C), 112.25 (1C), 64.06 (1C), 53.15 (1C), 45.93 (1C); $\mathrm{HRMS}$ (ESI) $\mathrm{m} / \mathrm{z}$ for $\mathrm{C}_{20} \mathrm{H}_{16} \mathrm{O}_{4} \mathrm{~N}_{2} \mathrm{BrClNa}[\mathrm{M}+\mathrm{Na}]^{+}$calcd 484.98742 , found 484.98654 .

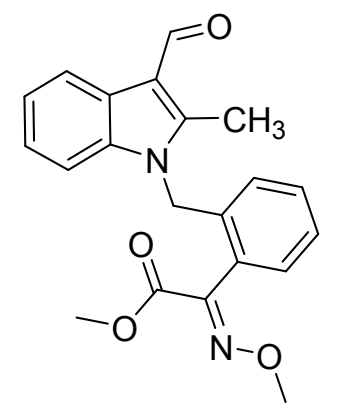

(E)-methyl-2-(2-((3-formyl-2-methyl-1H-indol-1-yl)methyl)phenyl)2-(methoxyimino)acetate (C4): Yield: 87 \%; yellow solid; m. p. 99.6 $101.1{ }^{\circ} \mathrm{C} ;{ }^{1} \mathrm{H}$ NMR $\left(400 \mathrm{MHz}, \mathrm{DMSO}-d_{6}\right) \delta 10.13(\mathrm{~s}, 1 \mathrm{H},-\mathrm{CH}=\mathrm{O}), 8.15$ (d, $J=6.3 \mathrm{~Hz}, 1 \mathrm{H}, \operatorname{Ar}-\mathrm{H}), 7.33$ (d, $J=7.4 \mathrm{~Hz}, 1 \mathrm{H}, \operatorname{Ar}-\mathrm{H}), 7.27$ (t, $J=$ $8.6 \mathrm{~Hz}, 3 \mathrm{H}, \mathrm{Ar}-\mathrm{H}), 7.22$ (d, J=2.3 Hz, 1H, Ar-H), $7.21-7.19$ (m, 1H, $\operatorname{Ar}-\mathrm{H}), 6.50(\mathrm{~d}, J=6.8 \mathrm{~Hz}, 1 \mathrm{H}, \mathrm{Ar}-\mathrm{H}), 5.25\left(\mathrm{~s}, 2 \mathrm{H},-\mathrm{NCH}_{2}-\right), 4.07$ (s, $\left.3 \mathrm{H},=\mathrm{N}-\mathrm{OCH}_{3}\right), 3.76\left(\mathrm{~s}, 3 \mathrm{H},-\mathrm{CO}-\mathrm{OCH}_{3}\right), 2.58\left(\mathrm{~s}, 3 \mathrm{H}\right.$, Indol 2- $\left.\mathrm{CH}_{3}\right) ;{ }^{13} \mathrm{C} \mathrm{NMR}(100 \mathrm{MHz}$, DMSO- $\left.d_{6}\right) \delta 185.13$ (1C), 162.94 (1C), 149.74 (1C), 148.91 (1C), 137.04 (1C), 135.03 (1C), 130.25 (1C), 129.47 (1C), 129.09 (1C), 127.84 (1C), 125.61 (1C), 125.48 (1C), 123.49 (1C), 122.98 (1C), 120.68 (1C), 114.44 (1C), 110.79 (1C), 64.07 (1C), 53.18 (1C), 44.76 (1C), 10.35 (1C); HRMS (ESI) m/z for $\mathrm{C}_{21} \mathrm{H}_{21} \mathrm{O}_{4} \mathrm{~N}_{2}[\mathrm{M}+\mathrm{H}]^{+}$calcd 365.14958, found 365.14874. 
(2) Characterization of products (D1-D28)

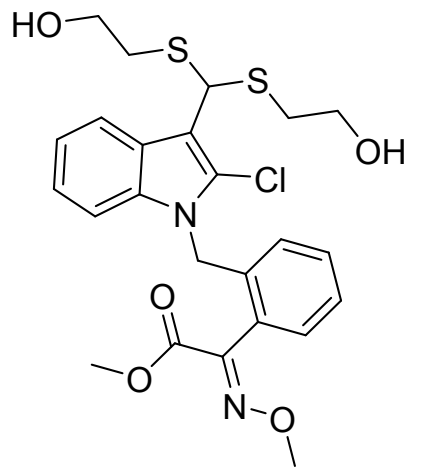

(E)-Methyl-2-(2-((3-(bis((2-hydroxyethyl)thio)methyl)-2-chlo ro-1H-indol-1-yl)methyl)phenyl)-2-(methoxyimino) acetate (D1): Yield: $76 \%$; white solid; m. p. 123.3 - $124.8^{\circ} \mathrm{C}$; ${ }^{1} \mathrm{H}$ NMR $\left(400 \mathrm{MHz}, \mathrm{DMSO}-d_{6}\right) \delta 7.90(\mathrm{~d}, J=7.8 \mathrm{~Hz}, 1 \mathrm{H}, \mathrm{Ar}-\mathrm{H}), 7.31(\mathrm{~d}$, $J=7.6 \mathrm{~Hz}, 1 \mathrm{H}, \mathrm{Ar}-\mathrm{H}), 7.25$ (d, $J=7.4 \mathrm{~Hz}, 2 \mathrm{H}, \mathrm{Ar}-\mathrm{H}), 7.23$ $7.15(\mathrm{~m}, 2 \mathrm{H}, \mathrm{Ar}-\mathrm{H}), 7.13(\mathrm{~d}, J=7.6 \mathrm{~Hz}, 1 \mathrm{H}, \mathrm{Ar}-\mathrm{H}), 6.40(\mathrm{~d}, J=$ 7.6 Hz, 1H, Ar-H), 5.51 (s, 1H, -SCHS-), 5.24 (s, 2H, - $\left.\mathrm{NCH}_{2}-\right), 4.85$ (s, 2H, -OH), 4.06 (s, $\left.3 \mathrm{H},-\mathrm{OCH}_{3}\right), 3.80\left(\mathrm{~s}, 3 \mathrm{H},-\mathrm{OCH}_{3}\right), 3.54\left(\mathrm{~d}, J=6.7 \mathrm{~Hz}, 4 \mathrm{H},-\mathrm{CH}_{2} \mathrm{O}-\right), 2.70-2.65(\mathrm{~m}, 2 \mathrm{H}$, $\left.-\mathrm{SCH}_{2}-\right), 2.63-2.58\left(\mathrm{~m}, 2 \mathrm{H},-\mathrm{SCH}_{2}-\right) .{ }^{13} \mathrm{C} \mathrm{NMR}\left(100 \mathrm{MHz}, \mathrm{DMSO}-d_{6}\right) \delta 163.07(\mathrm{~s}, 1 \mathrm{C})$, 148.86 (s, 1C), 135.91 (s, 1C), 135.52 (s, 1C), 130.19 (s, 1C), 129.24 (s, 1C), 129.00 (s, 1C), 127.73 (s, 1C), 125.35 (s, 1C), 124.79 (s, 1C), 123.85 (s, 1C), 123.06 (s, 1C), 120.67 (s, 1C), $120.55(\mathrm{~s}, 1 \mathrm{C}), 110.77(\mathrm{~s}, 1 \mathrm{C}), 110.65(\mathrm{~s}, 1 \mathrm{C}), 64.04$ (s, 1C), 61.14 (s, 2C), 53.26 (s, 1C), 45.04 (s, 1C), 44.89 (s, 1C), $35.48(\mathrm{~s}, 2 \mathrm{C})$. HRMS (ESI) $\mathrm{m} / \mathrm{z}$ for $\mathrm{C}_{24} \mathrm{H}_{27} \mathrm{O}_{5} \mathrm{~N}_{2} \mathrm{ClNaS}_{2}[\mathrm{M}+\mathrm{Na}]^{+}$ calcd 545.09421, found 545.09235.

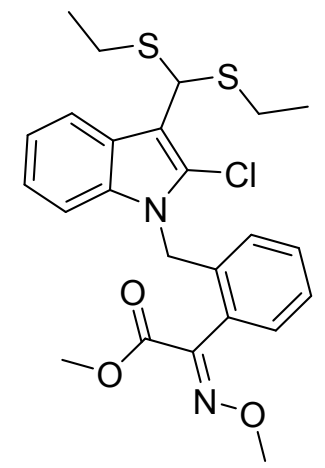

(E)-Methyl-2-(2-((3-(bis(ethylthio)methyl)-2-chloro-1H-indol-1-yl)m ethyl)phenyl)-2-(methoxyimino)acetate (D2): Yield: $78 \%$; white solid; m. p. $124.7-125.5^{\circ} \mathrm{C} ;{ }^{1} \mathrm{H}$ NMR (400 MHz, DMSO- $\left.d_{6}\right) \delta 7.89(\mathrm{~d}$, $J=7.8 \mathrm{~Hz}, 1 \mathrm{H}, \mathrm{Ar}-\mathrm{H}), 7.31(\mathrm{~d}, J=7.3 \mathrm{~Hz}, 1 \mathrm{H}, \mathrm{Ar}-\mathrm{H}), 7.26(\mathrm{~m}, 2 \mathrm{H}$, Ar-H), 7.23 - 7.14 (m, 2H, Ar-H), 7.12 (d, J=7.8 Hz, 1H, Ar-H), 6.41 (d, $J=7.5 \mathrm{~Hz}, 1 \mathrm{H}, \mathrm{Ar}-\mathrm{H}), 5.39$ (s, 1H, -SCHS-), 5.24 (s, 2H, - $\mathrm{NCH}_{2}-$ ), $4.04\left(\mathrm{~s}, 3 \mathrm{H},-\mathrm{OCH}_{3}\right), 3.79\left(\mathrm{~s}, 3 \mathrm{H},-\mathrm{OCH}_{3}\right), 2.59-2.51\left(\mathrm{~m}, J=12.2,7.3 \mathrm{~Hz}, 4 \mathrm{H},-\mathrm{SCH}_{2}-\right), 1.19$ (t, $\left.J=7.4 \mathrm{~Hz}, 6 \mathrm{H},-\mathrm{CH}_{3}\right) .{ }^{13} \mathrm{C} \mathrm{NMR}\left(100 \mathrm{MHz}, \mathrm{DMSO}-d_{6}\right) \delta 163.03(\mathrm{~s}, 1 \mathrm{C}), 148.83(\mathrm{~s}, 1 \mathrm{C})$, 135.98 (s, 1C), 135.56 (s, 1C), 130.09 (s, 1C), 129.32 (s, 1C), 129.05 (s, 1C), 127.76 (s, 1C), 125.37 (s, 1C), $124.78(\mathrm{~s}, 1 \mathrm{C}), 123.75$ (s, 1C), 123.05 (s, 1C), 120.62 (s, 1C), $120.52(\mathrm{~s}, 1 \mathrm{C})$, $110.80(\mathrm{~s}, 1 \mathrm{C}), 110.44$ (s, 1C), 64.01 (s, 1C), 53.22 (s, 1C), 44.98 (s, 1C), 43.49 (s, 1C), 26.70 (s, 2C), 14.97 (s, 2C). HRMS (ESI) $\mathrm{m} / \mathrm{z}$ for $\mathrm{C}_{24} \mathrm{H}_{27} \mathrm{O}_{3} \mathrm{~N}_{2} \mathrm{ClNaS}_{2}[\mathrm{M}+\mathrm{Na}]^{+}$calcd 513.10438, found 513.10291. 


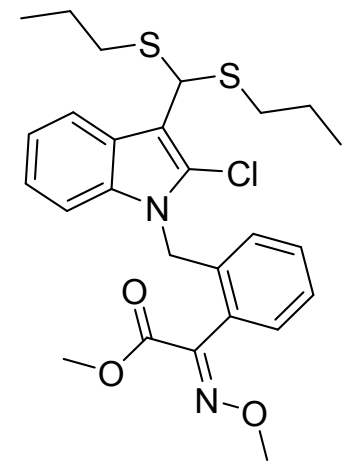

(E)-methyl-2-(2-((3-(bis(propylthio)methyl)-2-chloro-1H-indol-1-y l)methyl)phenyl)-2-(methoxyimino)acetate (D3): Yield: $73 \%$; white solid; m. p. $160.9-161.8{ }^{\circ} \mathrm{C} ;{ }^{1} \mathrm{H}$ NMR (400 MHz, DMSO- $\left.d_{6}\right) \delta$ $7.91(\mathrm{~d}, J=7.8 \mathrm{~Hz}, 1 \mathrm{H}, \mathrm{Ar}-\mathrm{H}), 7.31$ (d, $J=7.7 \mathrm{~Hz}, 1 \mathrm{H}, \mathrm{Ar}-\mathrm{H}), 7.29$ $7.23(\mathrm{~m}, 2 \mathrm{H}, \mathrm{Ar}-\mathrm{H}), 7.22$ - $7.14(\mathrm{~m}, 2 \mathrm{H}, \mathrm{Ar}-\mathrm{H}), 7.12$ (d, $J=7.4 \mathrm{~Hz}$ 1H, Ar-H), 6.37 (d, J=7.5 Hz, 1H, Ar-H), 5.39 (s, 1H, -SCHS-), 5.24 (s, $\left.2 \mathrm{H},-\mathrm{NCH}_{2}-\right), 4.03\left(\mathrm{~s}, 3 \mathrm{H},-\mathrm{OCH}_{3}\right), 3.79\left(\mathrm{~s}, 3 \mathrm{H},-\mathrm{OCH}_{3}\right), 2.90\left(\mathrm{~s}, 2 \mathrm{H},-\mathrm{SCH}_{2}-\right), 1.20-1.29$ $\left(\mathrm{m}, J=34.9,6.7 \mathrm{~Hz}, 12 \mathrm{H},-\mathrm{CH}_{2} \mathrm{CH}_{3}\right) \cdot{ }^{13} \mathrm{C} \mathrm{NMR}\left(100 \mathrm{MHz}, \mathrm{DMSO}-d_{6}\right) \delta 163.03(\mathrm{~s}, 1 \mathrm{C})$, 148.83 (s, 1C), 136.02 (s, 1C), 135.56 (s, 1C), 130.06 (s, 1C), 129.32 (s, 1C), 129.05 (s, 1C), 127.75 (s, 1C), 125.29 (s, 1C), 124.85 (s, 1C), 123.22 (s, 1C), 123.06 (s, 1C), 120.65 (s, 1C), 120.53 (s, 1C), 110.94 (s, 1C), 110.79 (s, 1C), 64.00 (s, 1C), 53.23 (s, 1C), 44.96 (s, 1C), 42.14 (s, 1C), 42.14 (s, 1C), 36.25 (s, 1C), 36.25 (s, 1C), 23.60 (s, 2C), 23.21 (s, 2C), 23.21 (s, 2C). HRMS (ESI) $\mathrm{m} / \mathrm{z}$ for $\mathrm{C}_{26} \mathrm{H}_{31} \mathrm{O}_{3} \mathrm{~N}_{2} \mathrm{ClNaS}_{2}[\mathrm{M}+\mathrm{Na}]^{+}$calcd 541.13568, found 541.13409 .<smiles>CO/N=C(/C(=O)OC)c1ccccc1Cn1c(Cl)c(C(SC(C)C)SC(C)C)c2ccccc21</smiles>

(E)-methyl-2-(2-((3-(bis(isopropylthio)methyl)-2-chloro-1H-indol-1yl)methyl)phenyl)-2-(methoxyimino)acetate (D4): Yield: $75 \%$; white solid; m. p. $134.5-135.7{ }^{\circ} \mathrm{C} ;{ }^{1} \mathrm{H}$ NMR (400 MHz, DMSO- $\left.d_{6}\right) \delta 7.90(\mathrm{~d}$, $J=7.9 \mathrm{~Hz}, 1 \mathrm{H}, \mathrm{Ar}-\mathrm{H}), 7.31(\mathrm{~d}, J=7.1 \mathrm{~Hz}, 1 \mathrm{H}, \mathrm{Ar}-\mathrm{H}), 7.28-7.23(\mathrm{~m}$, 2H, Ar-H), 7.22 - 7.14 (m, 2H, Ar-H), 7.12 (d, J=7.5 Hz, 1H, Ar-H), $6.37(\mathrm{~d}, J=8.1 \mathrm{~Hz}, 1 \mathrm{H}, \mathrm{Ar}-\mathrm{H}), 5.32$ (s, 1H, -SCHS-), 5.25 (s, 2H, $\left.-\mathrm{NCH}_{2}-\right), 4.05\left(\mathrm{~s}, 3 \mathrm{H},-\mathrm{OCH}_{3}\right), 3.79\left(\mathrm{~s}, 3 \mathrm{H},-\mathrm{OCH}_{3}\right), 2.60-2.44(\mathrm{~m}, 6 \mathrm{H}$, $\left.-\mathrm{CH}_{3}\right), 1.54\left(\mathrm{q}, J=7.3 \mathrm{~Hz}, 2 \mathrm{H},-\mathrm{SCH}_{2}-\right), 0.88\left(\mathrm{~m}, 6 \mathrm{H},-\mathrm{CH}_{3}\right) .{ }^{13} \mathrm{C}$ NMR $\left(100 \mathrm{MHz}, \mathrm{DMSO}-d_{6}\right)$ $\delta 163.03(\mathrm{~s}, 1 \mathrm{C}), 148.83(\mathrm{~s}, 1 \mathrm{C}), 135.97$ (s, 1C), $135.58(\mathrm{~s}, 1 \mathrm{C}), 130.03(\mathrm{~s}, 1 \mathrm{C}), 129.32(\mathrm{~s}$, 1C), $129.06(\mathrm{~s}, 1 \mathrm{C}), 127.76(\mathrm{~s}, 1 \mathrm{C}), 125.21(\mathrm{~s}, 1 \mathrm{C}), 124.74(\mathrm{~s}, 1 \mathrm{C}), 123.75(\mathrm{~s}, 1 \mathrm{C}), 123.08(\mathrm{~s}$, 1C), 120.66 (s, 1C), $120.53(\mathrm{~s}, 1 \mathrm{C}), 110.77$ (s, 1C), $110.50(\mathrm{~s}, 1 \mathrm{C}), 64.02$ (s, 1C), 53.23 (s,<smiles>CO/N=C(/C(=O)OC)c1ccccc1Cn1c(Cl)c(C(Sc2ccc(Cl)cc2)Sc2ccc(Cl)cc2)c2ccccc21</smiles>
1C), 44.92 (s, 1C), 44.15 (s, 1C), 34.73 (s, 3C), 22.76 (s, 3C), 13.73 (s, 3C). HRMS (ESI) $\mathrm{m} / \mathrm{z}$ for $\mathrm{C}_{26} \mathrm{H}_{31} \mathrm{O}_{3} \mathrm{~N}_{2} \mathrm{ClNaS}_{2}$ $[\mathrm{M}+\mathrm{Na}]^{+}$calcd 541.13568, found 541.13440.

(E)-methyl-2-(2-((3-(bis((4-chlorophenyl)thio)methyl)-2-c 
hloro-1H-indol-1-yl)methyl)phenyl)-2-(methoxyimino) acetate (D5): Yield: $81 \%$; white solid; m. p. $120.7-121.9^{\circ} \mathrm{C}$; ${ }^{1} \mathrm{H}$ NMR (400 MHz, DMSO- $\left.d_{6}\right) \delta 8.01(\mathrm{~d}, J=7.5 \mathrm{~Hz}, 1 \mathrm{H}$, Ar-H), 7.44 (d, $J=8.6 \mathrm{~Hz}, 4 \mathrm{H}, \mathrm{Ar}-\mathrm{H}), 7.37$ (d, $J=8.6 \mathrm{~Hz}, 4 \mathrm{H}, \mathrm{Ar}-\mathrm{H}), 7.34-7.29$ (m, 1H, Ar-H), $7.23(\mathrm{~d}, J=7.7 \mathrm{~Hz}, 1 \mathrm{H}, \mathrm{Ar}-\mathrm{H}), 7.19$ (d, $J=3.2 \mathrm{~Hz}, 1 \mathrm{H}, \mathrm{Ar}-\mathrm{H}), 7.18-7.14$ (m, 2H, Ar-H), 7.14 - 7.10 (m, 1H, Ar-H), 6.18 (s, 1H, Ar-H), 5.80 (d, J=7.8 Hz, 1H, -SCHS-), 5.13 (s, $\left.2 \mathrm{H},-\mathrm{NCH}_{2}-\right), 4.06\left(\mathrm{~s}, 3 \mathrm{H},-\mathrm{OCH}_{3}\right), 3.82\left(\mathrm{~s}, 3 \mathrm{H},-\mathrm{OCH}_{3}\right) \cdot{ }^{13} \mathrm{C}$ NMR (100 MHz, DMSO- $\left.d_{6}\right)$ $\delta 163.08$ (s, 1C), 148.83 (s, 1C), 135.55 (s, 1C), 135.20 (s, 1C), 134.10 (s, 4C), 133.27 (s, 2C), 133.07 (s, 3C), 129.91 (s, 1C), 129.48 (s, 4C), 129.16 (s, 1C), 128.91 (s, 1C), 127.64 (s, 1C), 125.08 (s, 1C), 124.58 (s, 1C), 123.29 (s, 1C), 120.96 (s, 1C), 120.39 (s, 1C), 110.81 (s, 1C), 108.37 (s, 1C), 64.04 (s, 1C), 53.28 (s, 1C), 51.13 (s, 1C), 44.58 (s, 1C). HRMS (ESI) $\mathrm{m} / \mathrm{z}$ for $\mathrm{C}_{32} \mathrm{H}_{25} \mathrm{O}_{3} \mathrm{~N}_{2} \mathrm{Cl}_{3} \mathrm{NaS}_{2}[\mathrm{M}+\mathrm{Na}]^{+}$calcd 677.02644 , found 677.02582 .

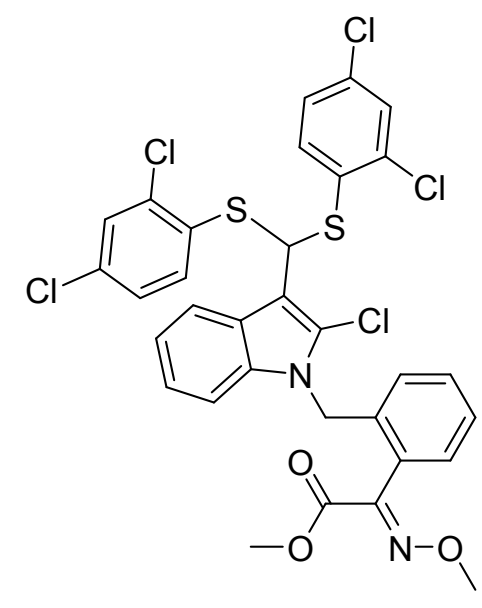

(E)-methyl-2-(2-((3-(bis((2,4-dichlorophenyl)thio)methyl)2-chloro-1H-indol-1-yl)methyl)phenyl)-2-(methoxyimino) acetate (D6): Yield: $73 \%$; white solid; m. p. 127.4 $128.5^{\circ} \mathrm{C}$

${ }^{1} \mathrm{H}$ NMR (400 MHz, DMSO- $\left.d_{6}\right) \delta 8.24(\mathrm{~d}, J=7.6 \mathrm{~Hz}, 1 \mathrm{H}$, Ar-H), 7.71 (s, 1H, Ar-H), 7.68 - 7.57 (m, 3H, Ar-H), 7.55 (d, $J=8.4 \mathrm{~Hz}, 1 \mathrm{H}, \mathrm{Ar}-\mathrm{H}), 7.48$ (d, $J=8.4 \mathrm{~Hz}, 1 \mathrm{H}, \mathrm{Ar}-\mathrm{H})$, $7.42(\mathrm{~d}, J=8.6 \mathrm{~Hz}, 1 \mathrm{H}, \mathrm{Ar}-\mathrm{H}), 7.36(\mathrm{~s}, 1 \mathrm{H}, \mathrm{Ar}-\mathrm{H}), 7.31(\mathrm{~d}, J$ $=3.8 \mathrm{~Hz}, 1 \mathrm{H}, \mathrm{Ar}-\mathrm{H}), 7.19(\mathrm{~d}, J=3.2 \mathrm{~Hz}, 1 \mathrm{H}, \mathrm{Ar}-\mathrm{H}), 7.16(\mathrm{~d}, J=6.9 \mathrm{~Hz}, 1 \mathrm{H}$, Ar-H), 7.10-7.13 (m, 1H, Ar-H), 6.39 (s, 1H, -SCHS-), 6.26 (d, $J=3.6 \mathrm{~Hz}, 1 \mathrm{H}$, Ar-H), 5.10 (s, 2H, $\left.-\mathrm{NCH}_{2}-\right), 3.85\left(\mathrm{~s}, 3 \mathrm{H},-\mathrm{OCH}_{3}\right), 3.75\left(\mathrm{~s}, 3 \mathrm{H},-\mathrm{OCH}_{3}\right) .{ }^{13} \mathrm{C}$ NMR $\left(100 \mathrm{MHz}, \mathrm{DMSO}-d_{6}\right) \delta$ 162.92 (s, 1C), 148.82 (s, 1C), 138.41 (s, 1C), 137.47 (s, 1C), 137.11 (s, 1C), 136.09 (s, 1C), 135.83 (s, 2C), 135.60 (s, 1C), 134.58 (s, 2C), 134.31 (s, 1C), 131.82 (s, 1C), 131.62 (s, 1C), 131.37 (s, 2C), 130.52 (s, 1C), 129.84 (s, 2C), 128.98 (s, 1C), 128.37 (s, 2C), 127.57 (s, 1C), 124.33 (s, 2C), 121.22 (s, 1C), 119.73 (s, 1C), 111.94 (s, 1C), 63.67 (s, 1C), 53.19 (s, 1C),

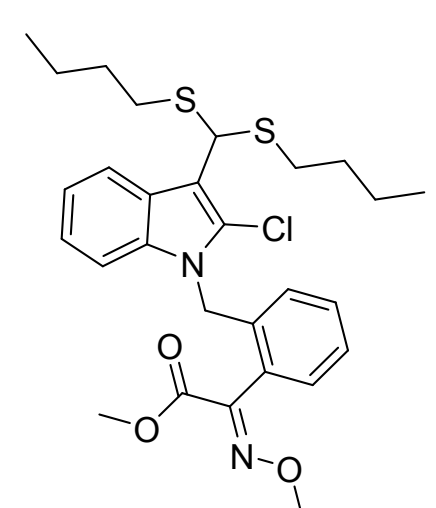
$50.61 \quad(\mathrm{~s}, \quad 1 \mathrm{C}), \quad 14.56 \quad(\mathrm{~s}, \quad 1 \mathrm{C}) . \quad \mathrm{HRMS} \quad(\mathrm{ESI}) \mathrm{m} / \mathrm{z}$ for $\mathrm{C}_{32} \mathrm{H}_{24} \mathrm{O}_{3} \mathrm{~N}_{2} \mathrm{Cl}_{5} \mathrm{~S}_{2}[\mathrm{M}+\mathrm{H}]^{+}$calcd 722.96655 , found 722.96948 . (E)-methyl-2-(2-((3-(bis(butylthio)methyl)-2-chloro-1H-indol 
-1-yl)methyl)phenyl)-2-(methoxyimino)acetate (D7): Yield: 85 \%; white solid; m. p. 76.9 $77.6{ }^{\circ} \mathrm{C} ;{ }^{1} \mathrm{H}$ NMR (400 MHz, DMSO- $\left.d_{6}\right) \delta 7.90(\mathrm{~d}, J=7.6 \mathrm{~Hz}, 1 \mathrm{H}, \mathrm{Ar}-\mathrm{H}), 7.31$ (d, $J=7.6 \mathrm{~Hz}$, 1H, Ar-H), $7.28-7.22(\mathrm{~m}, 2 \mathrm{H}, \mathrm{Ar}-\mathrm{H}), 7.20(\mathrm{~d}, J=8.5 \mathrm{~Hz}, 1 \mathrm{H}, \mathrm{Ar}-\mathrm{H}), 7.19-7.13(\mathrm{~m}, 1 \mathrm{H}$, Ar-H), 7.13 - 7.07 (m, 1H, Ar-H), 6.38 (d, $J=7.6$ Hz, 1H, Ar-H), 5.32 (s, 1H, -SCHS-), 5.25 (s, $\left.2 \mathrm{H},-\mathrm{NCH}_{2}-\right), 4.05\left(\mathrm{~s}, 3 \mathrm{H},-\mathrm{OCH}_{3}\right), 3.79\left(\mathrm{~s}, 3 \mathrm{H},-\mathrm{OCH}_{3}\right), 2.61-2.51\left(\mathrm{~m}, 4 \mathrm{H},-\mathrm{CH}_{2}\right.$ ) , 1.54-1.47 (m, 4H, $\left.-\mathrm{CH}_{2}-\right), 1.36-1.25\left(\mathrm{~m}, 4 \mathrm{H},-\mathrm{CH}_{2}-\right), 0.81$ (t, $\left.J=7.3 \mathrm{~Hz}, 6 \mathrm{H},-\mathrm{CH}_{3}\right) .{ }^{13} \mathrm{C}$ NMR (100 MHz, DMSO- $\left.d_{6}\right) \delta 163.04$ (s, 1C), 148.84 (s, 1C), 135.95 (s, 1C), 135.56 (s, 1C), 129.98 (s, 1C), 129.31 (s, 1C), 129.06 (s, 1C), 127.76 (s, 1C), 125.19 (s, 1C), 124.76 (s, 1C), 123.75 (s, 1C), 123.07 (s, 1C), 120.67 (s, 1C), 120.51 (s, 1C), 110.75 (s, 1C), 110.47 (s, 1C), 64.01 (s, 1C), 53.23 (s, 1C), 44.90 (s, 1C), 44.17 (s, 1C), 32.34 (s, 2C), 31.41 (s, 2C), 21.82 (s, 2C), 13.91 (s, 2C). HRMS (ESI) $\mathrm{m} / \mathrm{z}$ for $\mathrm{C}_{28} \mathrm{H}_{35} \mathrm{O}_{3} \mathrm{~N}_{2} \mathrm{ClNaS}_{2}[\mathrm{M}+\mathrm{Na}]^{+}$cacld 569.16698, found 569.16553 .

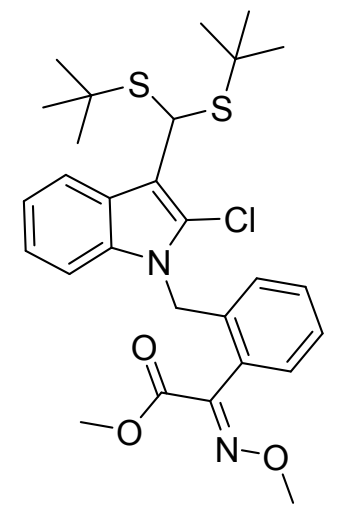

(E)-methyl-2-(2-((3-(bis(tert-butylthio)methyl)-2-chloro-1H-indol-1 -yl)methyl)phenyl)-2-(methoxyimino)acetate (D8): Yield: $79 \%$; white solid; m. p. $116.4-117.3{ }^{\circ} \mathrm{C} ;{ }^{1} \mathrm{H}$ NMR $\left(500 \mathrm{MHz}, \mathrm{CDCl}_{3}\right) \delta 8.14$ (d, $J=6.0 \mathrm{~Hz}, 1 \mathrm{H}, \mathrm{Ar}-\mathrm{H}), 7.29$ (t, $J=7.5 \mathrm{~Hz}, 1 \mathrm{H}, \mathrm{Ar}-\mathrm{H}), 7.19$ (d, $J=$ $7.7 \mathrm{~Hz}, 1 \mathrm{H}, \mathrm{Ar}-\mathrm{H}), 7.17-7.14(\mathrm{~m}, 1 \mathrm{H}, \mathrm{Ar}-\mathrm{H}), 7.13$ (d, $J=4.7 \mathrm{~Hz}, 1 \mathrm{H}$, Ar-H), 7.12 (d, J=2.4 Hz, 1H, Ar-H), 7.09 - 7.04 (m, 1H, Ar-H), 6.46 (d, $J=7.8 \mathrm{~Hz}, 1 \mathrm{H}, \mathrm{Ar}-\mathrm{H}), 5.42$ (s, 1H, -SCHS-), 5.22 (s, 2H, - $\mathrm{NCH}_{2}-$ ), $4.18\left(\mathrm{~s}, 3 \mathrm{H},-\mathrm{OCH}_{3}\right), 3.95\left(\mathrm{~s}, 3 \mathrm{H},-\mathrm{OCH}_{3}\right), 1.33\left(\mathrm{~s}, 18 \mathrm{H},-\mathrm{CH}_{3}\right) .{ }^{13} \mathrm{C} \mathrm{NMR}\left(100 \mathrm{MHz}, \mathrm{CDCl}_{3}\right)$ $\delta 163.42$ (s, 1C), 149.18 (s, 1C), 137.46 (s, 1C), 135.29 (s, 1C), 130.00 (s, 1C), 128.42 (s, 1C), 128.20 (s, 1C), 127.31 (s, 1C), 126.11 (s, 1C), 125.53 (s, 1C), 122.87 (s, 1C), 121.61 (s, 1C), 120.11 (s, 1C), 117.91 (s, 1C), 109.85 (s, 1C), 64.19 (s, 1C), 60.50 (s, 1C), 53.37 (s, 1C), 45.70 (s, 1C), 41.42 (s, 1C), 31.20 (s, 1C), 21.18 (s, 1C), 14.31 (s, 1C). HRMS (ESI) m/z for $\mathrm{C}_{28} \mathrm{H}_{35} \mathrm{O}_{3} \mathrm{~N}_{2} \mathrm{ClNaS}_{2}[\mathrm{M}+\mathrm{Na}]^{+}$cacld 569.16698, found 569.16698.

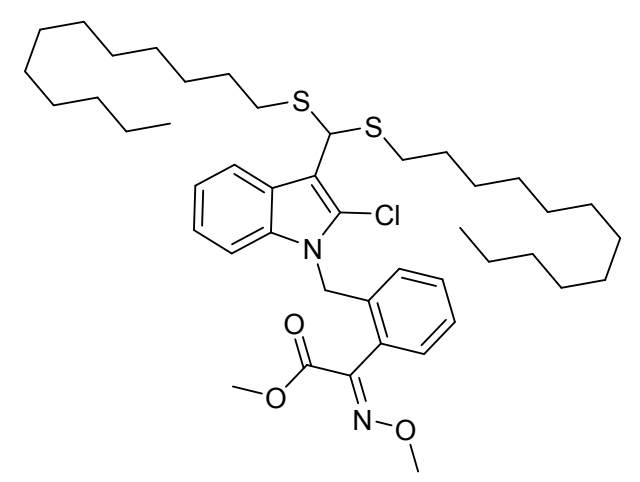

(E)-methyl-2-(2-((3-(bis(dodecylthio)methyl)-2-c hloro-1H-indol-1-yl)methyl)phenyl)-2-(methoxyi mino)acetate (D9): Yield: $83 \%$; white solid; m. p. 48.7 - $49.3{ }^{\circ} \mathrm{C} ;{ }^{1} \mathrm{H}$ NMR (400 MHz, Acetone- $\left.d_{6}\right) \delta$ 
$8.03(\mathrm{~d}, J=8.2 \mathrm{~Hz}, 1 \mathrm{H}, \mathrm{Ar}-\mathrm{H}), 7.37-7.32(\mathrm{~m}, 1 \mathrm{H}, \mathrm{Ar}-\mathrm{H}), 7.28-7.22$ (m, 2H, Ar-H), 7.19 (d, $J=7.3 \mathrm{~Hz}, 1 \mathrm{H}, \mathrm{Ar}-\mathrm{H}), 7.17$ (d, $J=6.7 \mathrm{~Hz}, 1 \mathrm{H}, \mathrm{Ar}-\mathrm{H}), 7.14-7.08$ (m, 1H, Ar-H), 6.52 (d, $J=$ $8.1 \mathrm{~Hz}, 1 \mathrm{H}, \mathrm{Ar}-\mathrm{H}), 5.40$ (s, 1H, -SCHS-), $5.31\left(\mathrm{~s}, 2 \mathrm{H},-\mathrm{NCH}_{2}-\right), 4.10\left(\mathrm{~s}, 3 \mathrm{H},-\mathrm{OCH}_{3}\right), 3.85(\mathrm{~s}$, $\left.3 \mathrm{H},-\mathrm{OCH}_{3}\right), 2.93\left(\mathrm{~s}, 26 \mathrm{H},-\mathrm{CH}_{2^{-}}\right), 2.66-2.54\left(\mathrm{~m}, 4 \mathrm{H},-\mathrm{CH}_{2}-\right), 2.04-2.06\left(\mathrm{~m}, 2 \mathrm{H},-\mathrm{CH}_{2^{-}}\right)$, $1.57-1.64\left(\mathrm{~m}, 4 \mathrm{H},-\mathrm{CH}_{2}-\right), 1.44-1.31\left(\mathrm{~m}, 8 \mathrm{H},-\mathrm{CH}_{2}-\right), 0.91-0.84(\mathrm{~m}, 6 \mathrm{H},-\mathrm{CH} 3) .{ }^{13} \mathrm{C} \mathrm{NMR}$ $\left(100 \mathrm{MHz}\right.$, Acetone- $\left.d_{6}\right) \delta 205.50(\mathrm{~s}, 1 \mathrm{C}), 163.00$ (s, 1C), 148.86 (s, 1C), 136.07 (s, 1C), 135.40 (s, 1C), 129.49 (s, 1C), 129.41 (s, 1C), 128.53 (s, 1C), 127.20 (s, 1C), 125.11 (s, 1C), 124.99 (s, 1C), 123.45 (s, 1C), 122.65 (s, 1C), 120.82 (s, 1C), 119.97 (s, 1C), $110.72(\mathrm{~s}, 1 \mathrm{C})$, 110.11 (s, 1C), 63.20 (s, 1C), 52.19 (s, 1C), 44.75 (s, 1C), 44.27 (s, 1C), 32.55 (s, 2C), 31.75 (s, 2C), 29.48 (s, 2C), 29.46 (s, 2C), 29.42 (s, 2C), 29.29 (s, 2C), 29.17 (s, 2C), 28.98 (s, 4C), 28.60 (s, 2C), 22.44 (s, 2C), 13.48 (s, 2C). HRMS (ESI) m/z for $\mathrm{C}_{44} \mathrm{H}_{68} \mathrm{O}_{3} \mathrm{~N}_{2} \mathrm{ClS}_{2}[\mathrm{M}+\mathrm{H}]^{+}$ cacld 771.43544 , found 771.43311 .

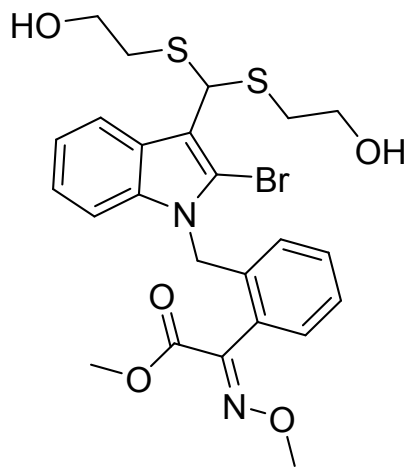

(E)-methyl-2-(2-((3-(bis((2-hydroxyethyl)thio)methyl)-2-bro mo-1H-indol-1-yl)methyl)phenyl)-2-(methoxyimino)acetate (D10): Yield: $87 \%$; white solid; m. p. $125.2-126.9^{\circ} \mathrm{C}$; ${ }^{1} \mathrm{H}$ NMR $\left(400 \mathrm{MHz}, \mathrm{DMSO}-d_{6}\right) \delta 7.92(\mathrm{~d}, J=7.4 \mathrm{~Hz}, 1 \mathrm{H}, \mathrm{Ar}-\mathrm{H}, \mathrm{Ar}-\mathrm{H})$, $7.30(\mathrm{~d}, J=6.9 \mathrm{~Hz}, 1 \mathrm{H}, \mathrm{Ar}-\mathrm{H}), 7.28-7.21(\mathrm{~m}, 2 \mathrm{H}, \mathrm{Ar}-\mathrm{H}), 7.17$ (t, $J=9.2 \mathrm{~Hz}, 2 \mathrm{H}, \mathrm{Ar}-\mathrm{H}), 7.10(\mathrm{~d}, J=7.8 \mathrm{~Hz}, 1 \mathrm{H}, \mathrm{Ar}-\mathrm{H}), 6.30$ (d, $J=8.1 \mathrm{~Hz}, 1 \mathrm{H}, \mathrm{Ar}-\mathrm{H}), 5.45$ (s, 1H, -SCHS-), 5.27 (s, 2H, - $\left.\mathrm{NCH}_{2}-\right), 4.81$ (s, 2H, -OH), $4.08\left(\mathrm{~s}, 3 \mathrm{H},-\mathrm{OCH}_{3}\right), 3.82\left(\mathrm{~s}, 3 \mathrm{H},-\mathrm{OCH}_{3}\right), 3.53\left(\mathrm{t}, J=7.1 \mathrm{~Hz}, 4 \mathrm{H},-\mathrm{SCH}_{2}-\right), 2.61-2.56(\mathrm{~m}$, 4H, - $\left.\mathrm{CH}_{2} \mathrm{O}-\right) .{ }^{13} \mathrm{C}$ NMR (100 MHz, DMSO- $\left.d_{6}\right) \delta 163.10$ (s, 1C), 148.91 (s, 1C), 137.17 (s, 1C), 135.58 (s, 1C), 130.16 (s, 1C), 129.16 (s, 1C), 128.95 (s, 1C), 127.64 (s, 1C), 125.46 (s, 1C), 125.12 (s, 1C), 123.01 (s, 1C), 120.61 (s, 1C), 120.35 (s, 1C), 114.48 (s, 1C), 113.78 (s, 1C), 110.91 (s, 1C), 64.06 (s, 1C), 61.15 (s, 2C), 53.28 (s, 1C), 46.36 (s, 1C), 46.22 (s, 1C), 35.53 (s, 2C). HRMS (ESI) $\mathrm{m} / \mathrm{z}$ for $\mathrm{C}_{24} \mathrm{H}_{27} \mathrm{O}_{5} \mathrm{~N}_{2} \mathrm{BrKS}_{2}[\mathrm{M}+\mathrm{K}]^{+}$calcd 605.01763 , found 605.01697 .

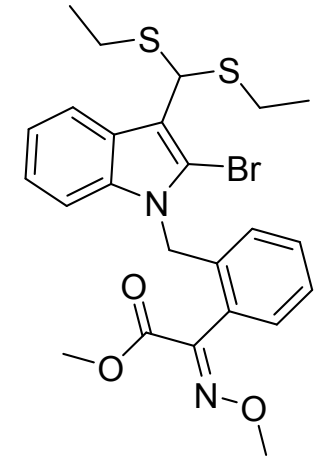

(E)-methyl-2-(2-((3-(bis(ethylthio)methyl)-2-bromo-1H-indol-1-yl)m ethyl)phenyl)-2-(methoxyimino)acetate (D11): Yield: 83 \%; white solid; m. p. $108.7-109.6{ }^{\circ} \mathrm{C} ;{ }^{1} \mathrm{H}$ NMR (400 MHz, DMSO- $\left.d_{6}\right) \delta 7.92(\mathrm{~d}$, 
$J=7.9 \mathrm{~Hz}, 1 \mathrm{H}, \mathrm{Ar}-\mathrm{H}), 7.34-7.28(\mathrm{~m}, 1 \mathrm{H}, \mathrm{Ar}-\mathrm{H}), 7.28-7.25(\mathrm{~m}, 1 \mathrm{H}, \mathrm{Ar}-\mathrm{H}), 7.24(\mathrm{~d}, J=5.8$ Hz, 1H, Ar-H), 7.18 (d, J=7.9 Hz, 1H, Ar-H), $7.17-7.12$ (m, 1H, Ar-H), 7.09 (t, $J=7.3 \mathrm{~Hz}$, 1H, Ar-H), 6.29 (d, $J=8.1 \mathrm{~Hz}, 1 \mathrm{H}, \mathrm{Ar}-\mathrm{H}), 5.36$ (s, 1H, -SCHS-), 5.27 (s, 2H, - $\left.\mathrm{NCH}_{2}-\right), 4.06$ (s, $\left.3 \mathrm{H},-\mathrm{OCH}_{3}\right), 3.81\left(\mathrm{~s}, 3 \mathrm{H},-\mathrm{OCH}_{3}\right), 2.58-2.53\left(\mathrm{~m}, 7.3 \mathrm{~Hz}, 4 \mathrm{H},-\mathrm{SCH}_{2}-\right), 1.20$ (t, $J=7.4 \mathrm{~Hz}$, $\left.6 \mathrm{H},-\mathrm{CH}_{3}\right) .{ }^{13} \mathrm{C}$ NMR (100 MHz, DMSO- $\left.d_{6}\right) \delta 163.06(\mathrm{~s}, 1 \mathrm{C}), 148.86(\mathrm{~s}, 1 \mathrm{C}), 137.22(\mathrm{~s}, 1 \mathrm{C})$, 135.63 (s, 1C), 130.07 (s, 1C), 129.22 (s, 1C), 129.01 (s, 1C), 127.68 (s, 1C), 125.43 (s, 1C), 125.09 (s, 1C), 123.01 (s, 1C), 120.57 (s, 1C), 120.33 (s, 1C), 114.39 (s, 1C), 113.57 (s, 1C), 110.94 (s, 1C), 64.04 (s, 1C), 53.26 (s, 1C), 46.28 (s, 1C), 44.75 (s, 1C), 26.71 (s, 2C), 15.10 (s, 2C). HRMS (ESI) $\mathrm{m} / \mathrm{z}$ for $\mathrm{C}_{24} \mathrm{H}_{27} \mathrm{O}_{3} \mathrm{~N}_{2} \mathrm{BrNaS}_{2}[\mathrm{M}+\mathrm{Na}]^{+}$calcd 557.05387, found 557.05292 .

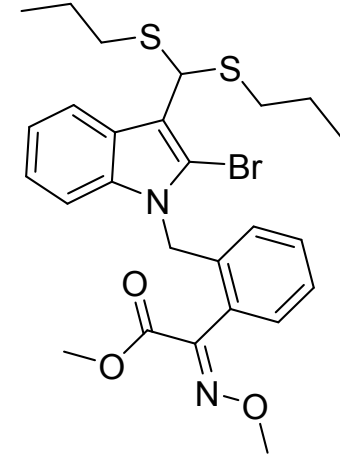

(E)-methyl-2-(2-((3-(bis(propylthio)methyl)-2-bromo-1H-indol-1yl)methyl)phenyl)-2-(methoxyimino)acetate (D12): Yield: $90 \%$; white solid; m. p. $131.5-132.3{ }^{\circ} \mathrm{C} ;{ }^{1} \mathrm{H}$ NMR $\left(400 \mathrm{MHz}, \mathrm{CDCl}_{3}\right) \delta$ $8.11(\mathrm{~d}, J=6.5 \mathrm{~Hz}, 1 \mathrm{H}, \mathrm{Ar}-\mathrm{H}), 7.31(\mathrm{t}, J=7.5 \mathrm{~Hz}, 1 \mathrm{H}, \mathrm{Ar}-\mathrm{H}), 7.24$ 7.17 (m, 2H, Ar-H), 7.13 (d, J=4.6 Hz, 2H, Ar-H), 7.12 - 7.07 (m, 1H, Ar-H), 6.50 (d, $J=7.7 \mathrm{~Hz}, 1 \mathrm{H}, \mathrm{Ar}-\mathrm{H}), 5.30$ (s, 1H, -SCHS-), 5.25 (s, $\left.2 \mathrm{H},-\mathrm{NCH}_{2}-\right), 4.16\left(\mathrm{~s}, 3 \mathrm{H},-\mathrm{OCH}_{3}\right), 3.93\left(\mathrm{~s}, 3 \mathrm{H},-\mathrm{OCH}_{3}\right), 2.61-2.50\left(\mathrm{~m}, 4 \mathrm{H},-\mathrm{SCH}_{2}-\right), 1.67$ $-1.58\left(\mathrm{~m}, 4 \mathrm{H},-\mathrm{CH}_{2}-\right), 0.95\left(\mathrm{t}, J=7.3 \mathrm{~Hz}, 6 \mathrm{H},-\mathrm{CH}_{3}\right) .{ }^{13} \mathrm{C} \mathrm{NMR}\left(100 \mathrm{MHz}, \mathrm{CDCl}_{3}\right) \delta 163.29$ (s, 1C), 149.06 (s, 1C), 137.28 (s, 1C), 135.18 (s, 1C), 129.98 (s, 1C), 128.44 (s, 1C), 128.21 (s, 1C), 127.34 (s, 1C), 125.54 (s, 1C), 125.51 (s, 1C), 122.83 (s, 1C), 120.95 (s, 1C), 120.22 (s, 1C), 114.02 (s, 1C), 113.34 (s, 1C), 110.03 (s, 1C), 64.12 (s, 1C), 53.29 (s, 1C), 46.31 (s, 1C), 45.58 (s, 1C), 35.05 (s, 2C), 22.90 (s, 2C), 13.63 (s, 2C). HRMS (ESI) m/z for $\mathrm{C}_{26} \mathrm{H}_{31} \mathrm{O}_{3} \mathrm{~N}_{2} \mathrm{BrNaS}_{2}[\mathrm{M}+\mathrm{Na}]^{+}$calcd 585.08517, found 585.08337.

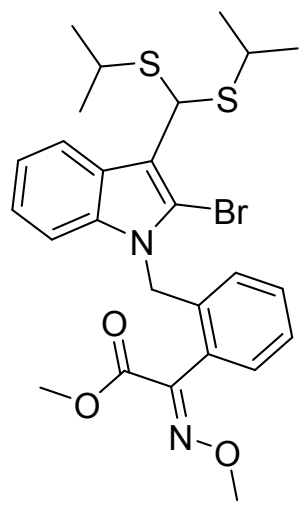

(E)-methyl-2-(2-((3-(bis(isopropylthio)methyl)-2-bromo-1H-indol-1yl)methyl)phenyl)-2-(methoxyimino)acetate (D13): Yield: $70 \%$; white solid; m. p. $94.8-95.9^{\circ} \mathrm{C} ;{ }^{1} \mathrm{H}$ NMR $\left(400 \mathrm{MHz}, \mathrm{CDCl}_{3}\right) \delta 8.19-$ $8.07(\mathrm{~m}, 1 \mathrm{H}, \mathrm{Ar}-\mathrm{H}), 7.31(\mathrm{t}, J=7.5 \mathrm{~Hz}, 1 \mathrm{H}, \mathrm{Ar}-\mathrm{H}), 7.22(\mathrm{~d}, J=6.9 \mathrm{~Hz}$, 1H, Ar-H), 7.19 (d, J=6.7 Hz, 1H, Ar-H), 7.17 - 7.11 (m, 2H, Ar-H), $7.10(\mathrm{~d}, J=3.5 \mathrm{~Hz}, 1 \mathrm{H}, \mathrm{Ar}-\mathrm{H}), 6.52(\mathrm{~d}, J=7.4 \mathrm{~Hz}, 1 \mathrm{H}, \mathrm{Ar}-\mathrm{H}), 5.40$ (s, 
1H, -SCHS-), 5.24 (s, 2H, -NCH2-), 4.15 (s, 3H, -OCH3), 3.92 (s, 3H, -OCH3), 2.97 (p, J= $6.7 \mathrm{~Hz}, 2 \mathrm{H},-\mathrm{SCH}-), 1.32$ (d, $J=6.8 \mathrm{~Hz}, 6 \mathrm{H},-\mathrm{CH} 3), 1.24$ (d, $J=6.8 \mathrm{~Hz}, 6 \mathrm{H},-\mathrm{CH} 3) .{ }^{13} \mathrm{C}$ NMR $\left(100 \mathrm{MHz}, \mathrm{CDCl}_{3}\right) \delta 163.28$ (s, 1C), 149.08 (s, 1C), 137.32 (s, 1C), 135.17 (s, 1C), 129.97 (s, 1C), $128.45(\mathrm{~s}, 1 \mathrm{C}), 128.19(\mathrm{~s}, 1 \mathrm{C}), 127.31(\mathrm{~s}, 1 \mathrm{C}), 125.72(\mathrm{~s}, 1 \mathrm{C}), 125.63(\mathrm{~s}, 1 \mathrm{C}), 122.77(\mathrm{~s}$, 1C), $121.01(\mathrm{~s}, 1 \mathrm{C}), 120.18(\mathrm{~s}, 1 \mathrm{C}), 114.50(\mathrm{~s}, 1 \mathrm{C}), 112.72(\mathrm{~s}, 1 \mathrm{C}), 109.98(\mathrm{~s}, 1 \mathrm{C}), 64.08$ (s, 1C), 53.25 (s, 1C), 46.34 (s, 1C), 43.51 (s, 1C), 36.16 (s, 2C), 23.49 (s, 2C), 23.04 (s, 2C). HRMS (ESI) $\mathrm{m} / \mathrm{z}$ for $\mathrm{C}_{26} \mathrm{H}_{31} \mathrm{O}_{3} \mathrm{~N}_{2} \mathrm{BrNaS}_{2}[\mathrm{M}+\mathrm{Na}]^{+}$calcd 585.08517, found 585.08325.

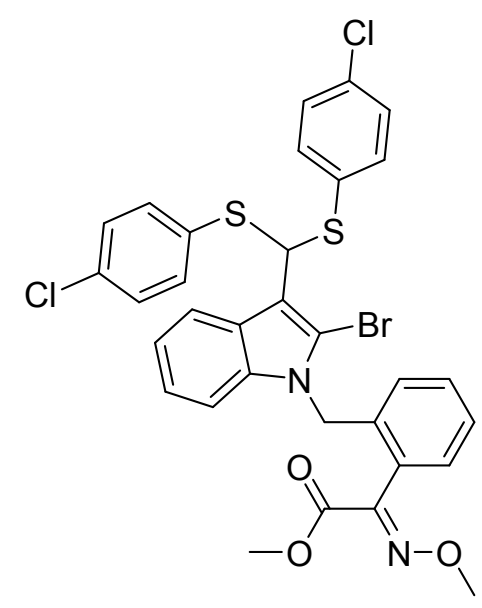

(E)-methyl-2-(2-((3-(bis((4-chlorophenyl)thio)methyl)-2-b romo-1H-indol-1-yl)methyl)phenyl)-2-(methoxyimino)ace tate (D14): Yield: 87 \%; white solid; m. p. 128.4 - $129.1^{\circ} \mathrm{C}$; ${ }^{1} \mathrm{H}$ NMR (400 MHz, DMSO- $\left.d_{6}\right) \delta 8.04(\mathrm{~d}, J=7.4 \mathrm{~Hz}, 1 \mathrm{H}$, Ar-H), 7.43 (d, $J=8.6 \mathrm{~Hz}, 4 \mathrm{H}, \mathrm{Ar}-\mathrm{H}), 7.38$ (d, $J=8.6 \mathrm{~Hz}$, 4H, Ar-H), $7.29-7.33$ (m, 1H, Ar-H), 7.23 (d, $J=7.7 \mathrm{~Hz}$, 1H, Ar-H), $7.19-7.14$ (m, 3H, Ar-H), 7.11 (d, $J=4.2 \mathrm{~Hz}$, 1H, Ar-H), 6.07 (s, 1H, Ar-H), 5.71 (d, $J=7.8 \mathrm{~Hz}, 1 \mathrm{H}$, -SCHS-), $5.16\left(\mathrm{~s}, 2 \mathrm{H},-\mathrm{NCH}_{2}-\right), 4.07\left(\mathrm{~s}, 3 \mathrm{H},-\mathrm{OCH}_{3}\right), 3.82\left(\mathrm{~s}, 3 \mathrm{H},-\mathrm{OCH}_{3}\right) .{ }^{13} \mathrm{C}$ NMR $(100$ MHz, DMSO- $\left.d_{6}\right) \delta 163.09$ (s, 1C), 148.83 (s, 1C), 136.78 (s, 1C), 135.26 (s, 1C), 134.24 (s, 4C), 133.34 (s, 2C), 133.03 (s, 2C), 129.88 (s, 1C), 129.51 (s, 4C), 129.08 (s, 1C), 128.88 (s, 1C), 127.58 (s, 1C), 125.21 (s, 1C), 124.43 (s, 1C), 123.25 (s, 1C), 120.79 (s, 1C), 120.30 (s, 1C), 115.87 (s, 1C), 111.46 (s, 1C), 110.98 (s, 1C), 64.06 (s, 1C), 53.32 (s, 1C), 52.76 (s, 1C), 45.94 (s, 1C). HRMS (ESI) m/z for $\mathrm{C}_{32} \mathrm{H}_{25} \mathrm{O}_{3} \mathrm{~N}_{2} \mathrm{BrCl}_{2} \mathrm{NaS}_{2}[\mathrm{M}+\mathrm{Na}]^{+}$calcd 720.97592, found 720.97418.<smiles>CO/N=C(/C(=O)OC)c1ccccc1Cn1c(Br)c(C(Sc2ccc(Cl)cc2Cl)Sc2ccc(Cl)cc2Cl)c2ccccc21</smiles>
(E)-methyl-2-(2-((3-(bis((2,4-dichlorophenyl)thio)methyl)2-bromo-1H-indol-1-yl)methyl)phenyl)-2-(methoxyimino) acetate (D15): Yield: 77 \%; white solid; m. p. 130.9 $131.7{ }^{\circ} \mathrm{C} ;{ }^{1} \mathrm{H}$ NMR $\left(500 \mathrm{MHz}, \mathrm{CDCl}_{3}\right) \delta 8.23(\mathrm{~d}, J=7.8 \mathrm{~Hz}$, 1H, Ar-H), 7.41 (s, 1H, Ar-H), 7.40 - 7.38 (m, 3H, Ar-H), $7.31(\mathrm{t}, J=7.4 \mathrm{~Hz}, 1 \mathrm{H}, \mathrm{Ar}-\mathrm{H}), 7.21(\mathrm{~d}, J=3.0 \mathrm{~Hz}, 1 \mathrm{H}$, Ar-H), 7.20 (d, $J=3.7 \mathrm{~Hz}, 1 \mathrm{H}, \mathrm{Ar}-\mathrm{H}), 7.18$ (s, 1H, Ar-H), 
$7.17(\mathrm{~d}, J=1.1 \mathrm{~Hz}, 1 \mathrm{H}, \mathrm{Ar}-\mathrm{H}), 7.11(\mathrm{~d}, J=2.3 \mathrm{~Hz}, 1 \mathrm{H}, \mathrm{Ar}-\mathrm{H}), 7.09$ (d, $J=2.3 \mathrm{~Hz}, 1 \mathrm{H}$, Ar-H), 7.06 (d, $J=8.3 \mathrm{~Hz}, 1 \mathrm{H}, \mathrm{Ar}-\mathrm{H}), 6.16$ (s, 1H, -SCHS-), 6.03 (d, $J=8.2 \mathrm{~Hz}, 1 \mathrm{H}, \mathrm{Ar}-\mathrm{H}$, Ar-H), 5.13 (s, 2H, $\left.-\mathrm{NCH}_{2}-\right), 4.16\left(\mathrm{~s}, 3 \mathrm{H},-\mathrm{OCH}_{3}\right), 3.94\left(\mathrm{~s}, 3 \mathrm{H},-\mathrm{OCH}_{3}\right) \cdot{ }^{13} \mathrm{C} \mathrm{NMR}(100 \mathrm{MHz}$, DMSO- $\left.d_{6}\right) \delta 162.91(\mathrm{~s}, 1 \mathrm{C}), 148.83(\mathrm{~s}, 1 \mathrm{C}), 138.42(\mathrm{~s}, 1 \mathrm{C}), 137.48(\mathrm{~s}, 1 \mathrm{C}), 136.10(\mathrm{~s}, 2 \mathrm{C})$, 135.59 (s, 1C), 134.58 (s, 2C), 134.09 (s, 1C), 131.83 (s, 1C), 131.38 (s, 2C), 130.53 (s, 1C), 129.83 (s, 2C), 129.39 (s, 1C), 128.99 (s, 1C), 128.83 (s, 1C), 128.36 (s, 2C), 127.56 (s, 1C), $124.95(\mathrm{~s}, 1 \mathrm{C}), 124.35$ (s, 2C), 121.21 (s, 1C), 119.75 (s, 1C), 111.92 (s, 1C), 63.67 (s, 1C), 56.49 (s, 1C), 53.17 (s, 1C), 19.02 (s, 1C). HRMS (ESI) m/z for $\mathrm{C}_{32} \mathrm{H}_{23} \mathrm{O}_{3} \mathrm{~N}_{2} \mathrm{BrCl}_{4} \mathrm{NaS}_{2}$ $[\mathrm{M}+\mathrm{Na}]^{+}$calcd 788.89798 , found 788.89691 .

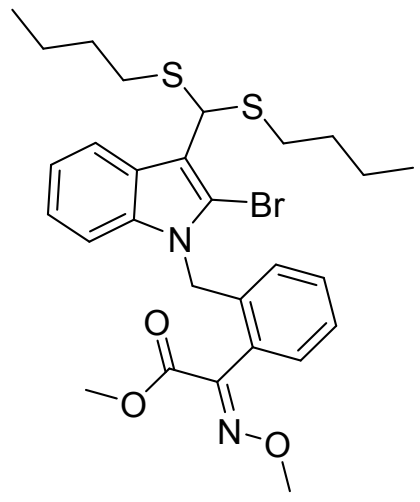

(E)-methyl-2-(2-((3-(bis(butylthio)methyl)-2-bromo-1H-indol -1-yl)methyl)phenyl)-2-(methoxyimino)acetate (D16): Yield: $87 \%$; white solid; m. p. 82.4 - $83.6{ }^{\circ} \mathrm{C}$; ${ }^{1} \mathrm{H}$ NMR $(400 \mathrm{MHz}$, DMSO- $\left.d_{6}\right) \delta 7.93(\mathrm{~d}, J=8.1 \mathrm{~Hz}, 1 \mathrm{H}, \mathrm{Ar}-\mathrm{H}), 7.31(\mathrm{t}, J=7.4 \mathrm{~Hz}$, 1H, Ar-H), $7.25-7.27$ (m, 1H, Ar-H), 7.19 (d, $J=17.6 \mathrm{~Hz}, 2 \mathrm{H}$, Ar-H), $7.16-7.11(\mathrm{~m}, 1 \mathrm{H}, \mathrm{Ar}-\mathrm{H}), 7.09$ (t, $J=7.3 \mathrm{~Hz}, 1 \mathrm{H}$, Ar-H), 6.27 (d, J=7.9 Hz, 1H, Ar-H), 5.30 (s, 1H, -SCHS-),

$5.27\left(\mathrm{~s}, 2 \mathrm{H},-\mathrm{NCH}_{2}-\right), 4.07\left(\mathrm{~s}, 3 \mathrm{H},-\mathrm{OCH}_{3}\right), 3.82\left(\mathrm{~s}, 3 \mathrm{H},-\mathrm{OCH}_{3}\right), 2.66-2.50\left(\mathrm{~m}, 4 \mathrm{H},-\mathrm{SCH}_{2}-\right)$, $1.51\left(\mathrm{q}, \mathrm{J}=7.3 \mathrm{~Hz}, 4 \mathrm{H},-\mathrm{CH}_{2^{-}}\right), 1.37-1.26\left(\mathrm{~m}, 4 \mathrm{H},-\mathrm{CH}_{2}-\right), 0.82\left(\mathrm{t}, \mathrm{J}=7.3 \mathrm{~Hz}, 6 \mathrm{H},-\mathrm{CH}_{3}\right)$. ${ }^{13} \mathrm{C}$ NMR (100 MHz, DMSO- $\left.d_{6}\right) \delta 163.07$ (s, 1C), 148.87 (s, 1C), 137.20 (s, 1C), 135.64 (s, 1C), $129.94(\mathrm{~s}, 1 \mathrm{C}), 129.23(\mathrm{~s}, 1 \mathrm{C}), 129.02(\mathrm{~s}, 1 \mathrm{C}), 127.68(\mathrm{~s}, 1 \mathrm{C}), 125.40(\mathrm{~s}, 1 \mathrm{C}), 124.92(\mathrm{~s}$ 1C), 123.03 (s, 1C), 120.62 (s, 1C), 120.31 (s, 1C), 114.44 (s, 1C), 113.59 (s, 1C), 110.89 (s, 1C), 64.05 (s, 1C), 53.27 (s, 1C), 46.21 (s, 1C), 45.38 (s, 1C), 32.34 (s, 2C), 31.54 (s, 2C), $21.82(\mathrm{~s}, 2 \mathrm{C}), 13.92\left(\mathrm{~s}, 2 \mathrm{C}\right.$ ). HRMS (ESI) $\mathrm{m} / \mathrm{z}$ for $\mathrm{C}_{28} \mathrm{H}_{35} \mathrm{O}_{3} \mathrm{~N}_{2} \mathrm{BrNaS}_{2}[\mathrm{M}+\mathrm{Na}]^{+}$cacld 613.11647, found 613.11505 .

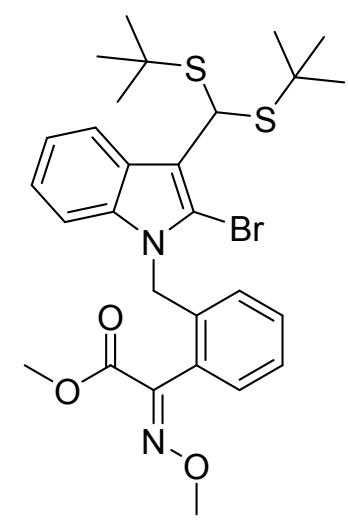

(E)-methyl-2-(2-((3-(bis(tert-butylthio)methyl)-2-bromo-1H-indol -1-yl)methyl)phenyl)-2-(methoxyimino)acetate (D17): Yield: $71 \%$; white solid; m. p. $89.2-90.8{ }^{\circ} \mathrm{C} ;{ }^{1} \mathrm{H}$ NMR $\left(500 \mathrm{MHz}, \mathrm{CDCl}_{3}\right) \delta 8.13$ $(\mathrm{d}, J=6.0 \mathrm{~Hz}, 1 \mathrm{H}, \mathrm{Ar}-\mathrm{H}), 7.29(\mathrm{t}, J=7.5 \mathrm{~Hz}, 1 \mathrm{H}, \mathrm{Ar}-\mathrm{H}), 7.18(\mathrm{~d}, J=$ $7.7 \mathrm{~Hz}, 1 \mathrm{H}, \mathrm{Ar}-\mathrm{H}), 7.16$ (d, J=7.7 Hz, 1H, Ar-H), 7.14 - 7.10 (m, 
2H, Ar-H), 7.06 - 7.08 (d, 1H, Ar-H), 6.45 (d, J= 7.8 Hz, 1H, Ar-H), 5.41 (s, 1H, Ar-H), 5.22 (s, 2H, Ar-H), 4.17 (s, 3H, Ar-H), 3.95 (s, 3H, Ar-H), 1.32 (s, 18H, Ar-H). ${ }^{13} \mathrm{C}$ NMR (100 $\left.\mathrm{MHz}, \mathrm{CDCl}_{3}\right) \delta 163.42$ (s, 1C), 149.18 (s, 1C), 137.46 (s, 1C), 135.29 (s, 1C), 130.00 (s, 1C), 128.42 (s, 1C), 128.20 (s, 1C), 127.31 (s, 1C), 126.11 (s, 1C), 125.53 (s, 1C), 122.87 (s, 1C), $121.61(\mathrm{~s}, 1 \mathrm{C}), 120.11(\mathrm{~s}, 1 \mathrm{C}), 117.91$ (s, 1C), 110.30 (s, 1C), 109.85 (s, 1C), 64.19 (s, 1C), 60.50 (s, 1C), 53.37 (s, 1C), 46.13 (s, 1C), 45.70 (s, 1C), 41.42 (s, 1C), 31.20 (s, 6C). HRMS (ESI) $\mathrm{m} / \mathrm{z}$ for $\mathrm{C}_{28} \mathrm{H}_{35} \mathrm{O}_{3} \mathrm{~N}_{2} \mathrm{BrNaS}_{2}[\mathrm{M}+\mathrm{Na}]^{+}$cacld 613.11647, found 613.11523 .

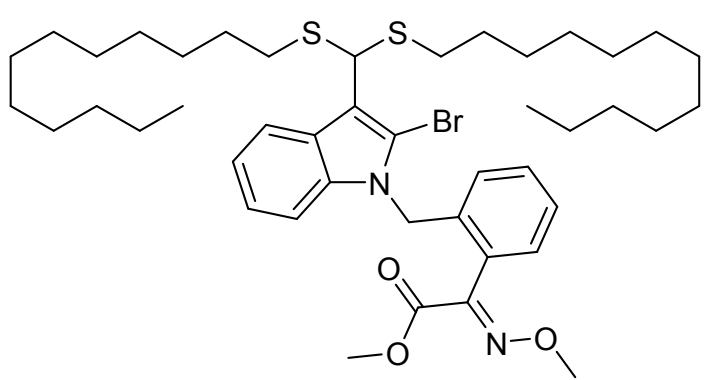

(E)-methyl-2-(2-((3-(bis(dodecylthio)methy l)-2-bromo-1H-indol-1-yl)methyl)phenyl)-2 -(methoxyimino)acetate (D18): Yield: $79 \%$; white solid; m. p. $36.7-37.9^{\circ} \mathrm{C} ;{ }^{1} \mathrm{H}$ NMR $\left(400 \mathrm{MHz}, \mathrm{CDCl}_{3}\right) \delta 8.01(\mathrm{~d}, J=7.4 \mathrm{~Hz}, 1 \mathrm{H}$, Ar-H), 7.28 (t, $J=7.5 \mathrm{~Hz}, 1 \mathrm{H}, \mathrm{Ar}-\mathrm{H}), 7.21$ (d, $J=6.4 \mathrm{~Hz}, 1 \mathrm{H}, \mathrm{Ar}-\mathrm{H}), 7.18(\mathrm{~d}, J=7.6 \mathrm{~Hz}, 1 \mathrm{H}$, Ar-H), 7.16 - 7.12 (m, 1H, Ar-H), 7.12 - 7.07 (m, 1H, Ar-H), 7.07 - 7.00 (m, 1H, Ar-H), 6.38 (d, $J=7.8 \mathrm{~Hz}, 1 \mathrm{H}, \mathrm{Ar}-\mathrm{H}), 5.33$ (s, 1H, -SCHS-), 5.29 (s, 2H, - $\left.\mathrm{NCH}_{2}-\right), 4.06$ (s, 3H, - $\mathrm{OCH}_{3}$ ), $3.81\left(\mathrm{~s}, 3 \mathrm{H},-\mathrm{OCH}_{3}\right), 2.57-2.51\left(\mathrm{~m}, 4 \mathrm{H},-\mathrm{SCH}_{2}-\right), 1.58-1.52(\mathrm{~m}, 4 \mathrm{H}), 1.22(\mathrm{~d}, J=2.7 \mathrm{~Hz}$, $\left.36 \mathrm{H},-\mathrm{CH}_{2}-\right), 0.85-0.79\left(\mathrm{~m}, 6 \mathrm{H},-\mathrm{CH}_{3}\right) .{ }^{13} \mathrm{C} \mathrm{NMR}\left(100 \mathrm{MHz}, \mathrm{CDCl}_{3}\right) \delta 163.30(\mathrm{~s}, 1 \mathrm{C})$, 149.03( s, 1C), 134.72 (s, 1C), 134.67 (s, 1C), 130.10 (s, 1C), 128.70 (s, 1C), 128.45 (s, 1C), $127.66(\mathrm{~s}, 1 \mathrm{C}), 126.50(\mathrm{~s}, 1 \mathrm{C}), 125.86(\mathrm{~s}, 1 \mathrm{C}), 125.65(\mathrm{~s}, 1 \mathrm{C}), 124.73(\mathrm{~s}, 1 \mathrm{C}), 123.56(\mathrm{~s}, 1 \mathrm{C})$, 113.92 (s, 1C), 111.50 (s, 1C), 110.66 (s, 1C), 64.17 (s, 1C), 53.35 (s, 1C), 45.24 (s, 1C), 44.22 (s, 1C), 33.11 (s, 2C), 32.03 (s, 2C), 29.78 (s, 2C), 29.76 (s, 2C), 29.72 (s, 2C), 29.64 (s, 2C), 29.47 (s, 2C), 29.44 (s, 2C), 29.34 (s, 2C), 29.04 (s, 2C), 22.81 (s, 2C), 14.26 (s, 2C). HRMS (ESI) $\mathrm{m} / \mathrm{z}$ for $\mathrm{C}_{44} \mathrm{H}_{66} \mathrm{O}_{3} \mathrm{~N}_{2} \mathrm{BrS}_{2}[\mathrm{M}-\mathrm{H}]^{+}$cacld 813.36927, found 813.37067.

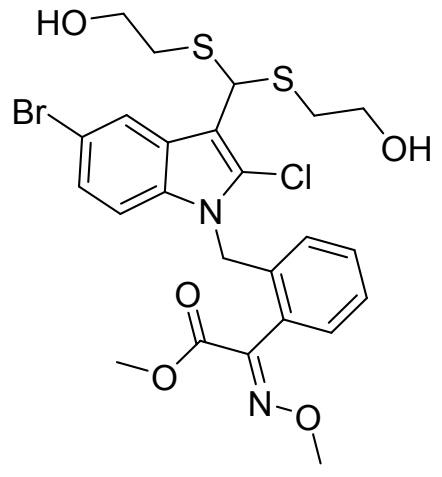

(E)-methyl-2-(2-((3-(bis((2-hydroxyethyl)thio)methyl)-5-bro mo-2-chloro-1H-indol-1-yl)methyl)phenyl)-2-(methoxyimin o)acetate (D19): Yield: 89 \%; white solid; m. p. 93.1 - $94.6^{\circ} \mathrm{C}$; ${ }^{1} \mathrm{H}$ NMR (400 MHz, DMSO- $\left.d_{6}\right) \delta 8.04(\mathrm{~d}, J=1.9 \mathrm{~Hz}, 1 \mathrm{H}$, Ar-H), 7.32 (d, $J=5.9 \mathrm{~Hz}, 2 \mathrm{H}, \mathrm{Ar}-\mathrm{H}), 7.26$ (t, $J=8.0 \mathrm{~Hz}, 2 \mathrm{H}$, Ar-H), 7.20 (d, $J=8.8 \mathrm{~Hz}, 1 \mathrm{H}, \mathrm{Ar}-\mathrm{H}), 6.41$ (d, $J=7.5 \mathrm{~Hz}, 1 \mathrm{H}$, 
Ar-H), 5.50 (s, 1H, -SCHS-), 5.26 (s, 2H, - $\mathrm{NCH}_{2}-$ ), 4.85 (s, 2H, -OH), 4.04 (s, 3H, $-\mathrm{OCH}_{3}$ ), $3.79\left(\mathrm{~s}, 3 \mathrm{H},-\mathrm{OCH}_{3}\right), 3.56-3.52\left(\mathrm{~m}, 4 \mathrm{H},-\mathrm{SCH}_{2}-\right), 2.71-2.66\left(\mathrm{~m}, 2 \mathrm{H},-\mathrm{CH}_{2} \mathrm{O}-\right), 2.63-2.56$ (m, 2H, - $\left.\mathrm{CH}_{2} \mathrm{O}-\right) .{ }^{13} \mathrm{C}$ NMR (100 MHz, DMSO-d 6 ) $\delta 162.99$ (s, 1C), 148.78 (s, 1C), 135.15 (s, 1C), 134.73 (s, 1C), 130.24 (s, 1C), 129.31 (s, 1C), 129.11 (s, 1C), 127.86 (s, 1C), 126.33 (s, 1C), 125.60 (s, 1C), 125.47 (s, 1C), 125.40 (s, 1C), 122.60 (s, 1C), 113.16 (s, 1C), 113.08 (s, 1C), 110.50 (s, 1C), 64.04 (s, 1C), 61.09 (s, 2C), 53.24 (s, 1C), 45.25 (s, 1C), 44.65 (s, 1C), 35.51 (s, 2C). HRMS (ESI) $\mathrm{m} / \mathrm{z}$ for $\mathrm{C}_{24} \mathrm{H}_{26} \mathrm{O}_{5} \mathrm{~N}_{2} \mathrm{BrClNaS}_{2}[\mathrm{M}+\mathrm{Na}]^{+}$calcd 623.00472, found 623.00244 .

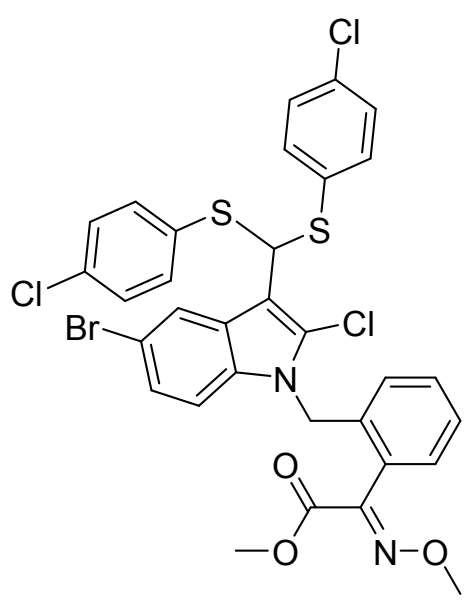

(E)-methyl-2-(2-((3-(bis((4-chlorophenyl)thio)methyl)-5-b romo-2-chloro-1H-indol-1-yl)methyl)phenyl)-2-(methoxyi mino)acetate (D20): Yield: $88 \%$; white solid; m. p. 65.9 $66.5{ }^{\circ} \mathrm{C} ;{ }^{1} \mathrm{H}$ NMR $\left(400 \mathrm{MHz}, \mathrm{CDCl}_{3}\right) \delta 8.20(\mathrm{~d}, J=1.8 \mathrm{~Hz}$, 1H, Ar-H), 7.31 (d, $J=8.6 \mathrm{~Hz}, 5 \mathrm{H}, \mathrm{Ar}-\mathrm{H}), 7.23$ (d, $J=2.0$ $\mathrm{Hz}, 1 \mathrm{H}, \mathrm{Ar}-\mathrm{H}), 7.21$ (d, $J=2.1 \mathrm{~Hz}, 3 \mathrm{H}, \mathrm{Ar}-\mathrm{H}), 7.19$ (t, $J=$ $2.1 \mathrm{~Hz}, 2 \mathrm{H}, \mathrm{Ar}-\mathrm{H}), 7.16$ (d, $J=7.4 \mathrm{~Hz}, 1 \mathrm{H}, \mathrm{Ar}-\mathrm{H}), 6.90$ (d, $J$ $=8.7 \mathrm{~Hz}, 1 \mathrm{H}, \mathrm{Ar}-\mathrm{H}), 6.05(\mathrm{~d}, J=1.2 \mathrm{~Hz}, 1 \mathrm{H}, \mathrm{Ar}-\mathrm{H}), 5.77(\mathrm{~s}$, 1H, -SCHS-), 5.06 (s, 2H, - $\left.\mathrm{NCH}_{2}-\right), 4.13$ (s, 3H, - $\left.\mathrm{OCH}_{3}\right), 3.92\left(\mathrm{~s}, 3 \mathrm{H},-\mathrm{OCH}_{3}\right) .{ }^{13} \mathrm{C}$ NMR $(100$ $\left.\mathrm{MHz}, \mathrm{CDCl}_{3}\right) \delta 163.26$ (s, 1C), 148.91 (s, 1C), 134.67 (s, 4C), 134.49 (s, 2C), $134.22(\mathrm{~s}, 2 \mathrm{C})$, 132.47 (s, 2C), 130.11 (s, 1C), 129.06 (s, 4C), 128.46 (s, 1C), 128.28 (s, 1C), 127.63 (s, 1C), 126.05 (s, 1C), 125.96 (s, 1C), 125.75 (s, 1C), 125.07 (s, 1C), 123.15 (s, 1C), 114.12 (s, 1C), 111.60 (s, 1C), 108.85 (s, 1C), 64.13 (s, 1C), 53.34 (s, 1C), 52.80 (s, 1C), 44.91 (s, 1C). HRMS (ESI) $\mathrm{m} / \mathrm{z}$ for $\mathrm{C}_{32} \mathrm{H}_{24} \mathrm{O}_{3} \mathrm{~N}_{2} \mathrm{BrCl}_{3} \mathrm{NaS}_{2}[\mathrm{M}+\mathrm{Na}]^{+}$calcd 754.93695, found 754.93494.

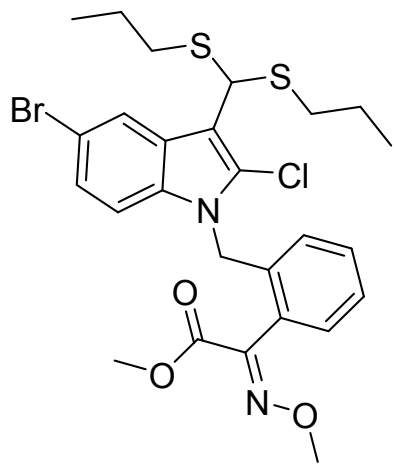

(E)-methyl-2-(2-((3-(bis(propylthio)methyl)-5-bromo-2-chloro -1H-indol-1-yl)methyl)phenyl)-2-(methoxyimino)acetate (D21): Yield: $88 \%$; white solid; m. p. 109.2 - $111.8{ }^{\circ} \mathrm{C} ;{ }^{1} \mathrm{H}$ NMR $\left(400 \mathrm{MHz}, \mathrm{DMSO}-d_{6}\right) \delta 8.05(\mathrm{~d}, J=1.9 \mathrm{~Hz}, 1 \mathrm{H}, \mathrm{Ar}-\mathrm{H}), 7.36-$ 7.33 (m, 1H, Ar-H), 7.32 (d, $J=2.0 \mathrm{~Hz}, 1 \mathrm{H}, \mathrm{Ar}-\mathrm{H}), 7.30-7.26$ (m, 1H, Ar-H), 7.26 (s, 1H, Ar-H), $7.21(\mathrm{~d}, J=8.8 \mathrm{~Hz}, 1 \mathrm{H}$, Ar-H), 6.40 (d, J=7.0 Hz, 1H, Ar-H), 5.31 (s, 1H, -SCHS-), 5.27 (s, 2H, - $\left.\mathrm{NCH}_{2}-\right), 4.03$ (s, 
$\left.3 \mathrm{H},-\mathrm{OCH}_{3}\right), 3.78\left(\mathrm{~s}, 3 \mathrm{H},-\mathrm{OCH}_{3}\right), 2.59-2.45\left(\mathrm{~m}, 4 \mathrm{H},-\mathrm{SCH}_{2}-\right), 1.54(\mathrm{q}, J=7.3 \mathrm{~Hz}, 4 \mathrm{H}$, $\left.-\mathrm{CH}_{2}-\right), 0.89\left(\mathrm{t}, J=7.3 \mathrm{~Hz}, 6 \mathrm{H},-\mathrm{CH}_{3}\right) .{ }^{13} \mathrm{C}$ NMR (100 MHz, DMSO- $\left.d_{6}\right) \delta 162.95$ (s, 1C), 148.73 (s, 1C), 135.20 (s, 1C), 134.78 (s, 1C), 130.09 (s, 1C), 129.36 (s, 1C), 129.17 (s, 1C), 127.88 (s, 1C), 126.26 (s, 1C), 125.62 (s, 1C), 125.37 (s, 1C), 125.29 (s, 1C), 122.61 (s, 1C), 113.13 (s, 1C), 110.31 (s, 1C), 64.01 (s, 1C), 55.38 (s, 1C), 53.21 (s, 1C), 45.28 (s, 1C), 43.69 (s, 1C), 34.71 (s, 2C), 22.71 (s, 2C), 13.71 (s, 2C). HRMS (ESI) m/z for $\mathrm{C}_{26} \mathrm{H}_{30} \mathrm{O}_{3} \mathrm{~N}_{2}$ $\mathrm{BrClNaS}_{2}[\mathrm{M}+\mathrm{Na}]^{+}$calcd 619.04620, found 619.04443.

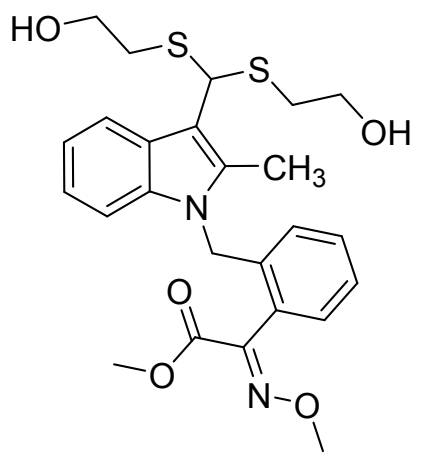

(E)-methyl-2-(2-((3-(bis((2-hydroxyethyl)thio)methyl)-2-met hyl-1H-indol-1-yl)methyl)phenyl)-2-(methoxyimino) acetate (D22): Yield: $86 \%$; yellow solid; m. p. $124-125{ }^{\circ} \mathrm{C}$; ${ }^{1} \mathrm{H}$ NMR $\left(500 \mathrm{MHz}, \mathrm{CDCl}_{3}\right) \delta 7.90(\mathrm{~d}, J=7.8 \mathrm{~Hz}, 1 \mathrm{H}, \mathrm{Ar}-\mathrm{H}), 7.36-7.32$ (m, 1H, Ar-H), 7.29 (d, $J=8.6 \mathrm{~Hz}, 1 \mathrm{H}, \mathrm{Ar}-\mathrm{H}), 7.28$ - 7.24 (m, 1H, Ar-H), 7.21 (d, J=7.9 Hz, 1H, Ar-H), $7.13-7.09$ (m, 1H, Ar-H), 7.09 - 7.05 (m, 1H, Ar-H), 6.23 (d, $J=7.7$ Hz, 1H, Ar-H), 5.66 (s, 1H, -SCHS-), 5.20 (s, $\left.2 \mathrm{H},-\mathrm{NCH}_{2}-\right), 4.85(\mathrm{~s}, 2 \mathrm{H},-\mathrm{OH}), 4.14\left(\mathrm{~s}, 3 \mathrm{H},-\mathrm{OCH}_{3}\right), 3.87\left(\mathrm{~s}, 3 \mathrm{H},-\mathrm{OCH}_{3}\right), 3.60$ - $3.52(\mathrm{~m}$, $\left.4 \mathrm{H},-\mathrm{CH}_{2}-\right), 2.66-2.72\left(\mathrm{~m}, 2 \mathrm{H},-\mathrm{CH}_{2}-\right), 2.57-2.63(\mathrm{~m}, 2 \mathrm{H}), 2.34\left(\mathrm{~s}, 3 \mathrm{H},-\mathrm{CH}_{3}\right) .{ }^{13} \mathrm{C}^{\mathrm{N} M R}$ $\left(100 \mathrm{MHz}, \mathrm{CDCl}_{3}\right) \delta 167.98$ (s, 1C), 153.91 (s, 1C), 141.67 (s, 1C), 141.25 (s, 1C), 139.85 (s, 1C), 134.97 (s, 1C), 133.99 (s, 1C), 133.66 (s, 1C), 132.28 (s, 1C), 131.03 (s, 1C), 129.86 (s, 1C), 126.37 (s, 1C), 125.32 (s, 1C), 124.23 (s, 1C), 115.20 (s, 1C), 114.67 (s, 1C), 68.88 (s, 1C), 66.23 (s, 2C), 58.10 (s, 1C), 50.49 (s, 1C), 49.00 (s, 1C), 40.22 (s, 2C), 15.26 (s, 1C). HRMS (ESI) $\mathrm{m} / \mathrm{z}$ for $\mathrm{C}_{25} \mathrm{H}_{30} \mathrm{O}_{5} \mathrm{~N}_{2} \mathrm{NaS}_{2}[\mathrm{M}+\mathrm{Na}]^{+}$calcd 525.14883, found 525.14795.

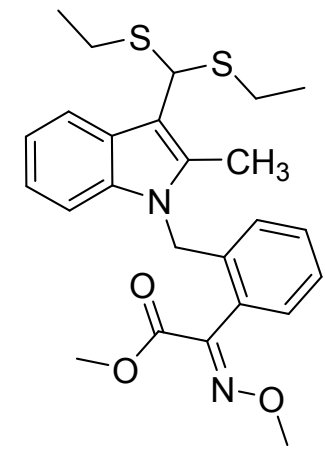

(E)-methyl-2-(2-((3-(bis(ethylthio)methyl)-2-methyl-1H-indol-1-yl) methyl)phenyl)-2-(methoxyimino)acetate (D23): Yield: $95 \%$; white solid; m. p. $116-117^{\circ} \mathrm{C} ;{ }^{1} \mathrm{H}$ NMR $\left(500 \mathrm{MHz}, \mathrm{CDCl}_{3}\right) \delta 7.96(\mathrm{~d}, J=$ $6.7 \mathrm{~Hz}, 1 \mathrm{H}, \mathrm{Ar}-\mathrm{H}), 7.29$ (t, $J=7.5 \mathrm{~Hz}, 1 \mathrm{H}, \mathrm{Ar}-\mathrm{H}), 7.19$ (d, $J=7.7 \mathrm{~Hz}$, 1H, Ar-H), 7.16 (d, $J=6.8 \mathrm{~Hz}, 1 \mathrm{H}, \mathrm{Ar}-\mathrm{H}), 7.16-7.06$ (m, 3H, Ar-H), 6.41 (d, $J=7.8 \mathrm{~Hz}, 1 \mathrm{H}, \mathrm{Ar}-\mathrm{H}), 5.38$ (s, 1H, -SCHS-), 5.12 (s, 2H, $\left.-\mathrm{NCH}_{2}-\right), 4.16\left(\mathrm{~s}, 3 \mathrm{H},-\mathrm{OCH}_{3}\right), 3.92\left(\mathrm{~s}, 3 \mathrm{H},-\mathrm{OCH}_{3}\right), 2.59$ (q, $\left.J=7.1 \mathrm{~Hz}, 4 \mathrm{H},-\mathrm{SCH}_{2}-\right), 2.33$ (s, $\left.3 \mathrm{H},-\mathrm{CH}_{3}\right), 1.23\left(\mathrm{t}, J=7.4 \mathrm{~Hz}, 6 \mathrm{H},-\mathrm{CH}_{3}\right) .{ }^{13} \mathrm{C} \mathrm{NMR}\left(100 \mathrm{MHz}, \mathrm{CDCl}_{3}\right) \delta 163.32(\mathrm{~s}, 1 \mathrm{C})$, 
149.26 (s, 1C), 136.69 (s, 1C), 135.80 (s, 1C), 133.93 (s, 1C), 130.04 (s, 1C), 128.43 (s, 1C), 128.05 (s, 1C), 127.19 (s, 1C), 126.38 (s, 1C), 125.48 (s, 1C), 121.51 (s, 1C), 120.03 (s, 1C), $119.60(\mathrm{~s}, 1 \mathrm{C}), 110.30$ (s, 1C), 109.18 (s, 1C), 64.07 (s, 1C), 53.27 (s, 1C), 44.27 (s, 1C), 44.13 (s, 1C), 26.91 (s, 2C), 14.55 (s, 2C), 10.60 (s, 1C). HRMS (ESI) m/z for $\mathrm{C}_{25} \mathrm{H}_{30} \mathrm{O}_{3} \mathrm{~N}_{2} \mathrm{NaS}_{2}[\mathrm{M}+\mathrm{Na}]^{+}$calcd 493.15901, found 493.15787.

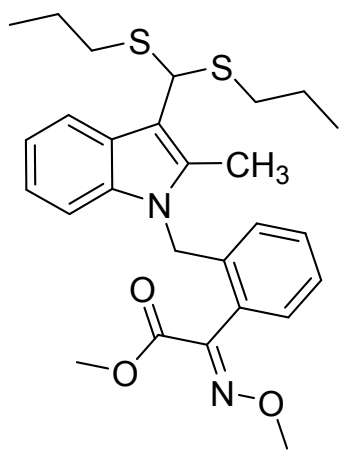

(E)-methyl-2-(2-((3-(bis(propylthio)methyl)-2-methyl-1H-indol-1yl)methyl)phenyl)-2-(methoxyimino)acetate (D24): Yield: $92 \%$; white solid; m. p. $113-114{ }^{\circ} \mathrm{C} ;{ }^{1} \mathrm{H}$ NMR $\left(500 \mathrm{MHz}, \mathrm{CDCl}_{3}\right) \delta 8.00-$ 7.91 (m, 1H, Ar-H), 7.29 (t, $J=7.5 \mathrm{~Hz}, 1 \mathrm{H}, \mathrm{Ar}-\mathrm{H}), 7.17$ (t, $J=8.0$ $\mathrm{Hz}, 2 \mathrm{H}, \mathrm{Ar}-\mathrm{H}), 7.11$ (d, $J=2.9 \mathrm{~Hz}, 3 \mathrm{H}, \mathrm{Ar}-\mathrm{H}), 6.39$ (d, $J=7.4 \mathrm{~Hz}$, 1H, Ar-H), 5.32 (s, 1H, -SCHS-), 5.12 (s, 2H, - $\left.\mathrm{NCH}_{2}-\right), 4.17$ (s, 3H, $\left.\left.-\mathrm{OCH}_{3}\right), 3.93\left(\mathrm{~s}, 3 \mathrm{H},-\mathrm{OCH}_{3}\right)\right), 2.58-2.49\left(\mathrm{~m}, 4 \mathrm{H},-\mathrm{SCH}_{2}-\right), 2.32\left(\mathrm{~s}, 3 \mathrm{H},-\mathrm{CH}_{3}\right), 1.59-1.62$ $\left(\mathrm{m}, 2 \mathrm{H},-\mathrm{CH}_{2}-\right), 1.58-1.55\left(\mathrm{~m}, 2 \mathrm{H},-\mathrm{CH}_{2}-\right), 0.97-0.90\left(\mathrm{~m}, 6 \mathrm{H},-\mathrm{CH}_{3}\right) .{ }^{13} \mathrm{C} \mathrm{NMR}(100 \mathrm{MHz}$, $\left.\mathrm{CDCl}_{3}\right) \delta 163.33(\mathrm{~s}, 1 \mathrm{C}), 149.24(\mathrm{~s}, 1 \mathrm{C}), 136.67(\mathrm{~s}, 1 \mathrm{C}), 135.83(\mathrm{~s}, 1 \mathrm{C}), 133.88(\mathrm{~s}, 1 \mathrm{C})$, 130.03 (s, 1C), 128.40 (s, 1C), 128.05 (s, 1C), 127.19 (s, 1C), 126.33 (s, 1C), 125.41 (s, 1C), 121.51 (s, 1C), 120.11 (s, 1C), 119.58 (s, 1C), 110.41 (s, 1C), 109.13 (s, 1C), 64.09 (s, 1C), $53.30(\mathrm{~s}, 1 \mathrm{C}), 44.91$ (s, 1C), 44.25 (s, 1C), 35.02 (s, 2C), 22.84 (s, 2C), 13.67 (s, 2C), 10.57 (s, 1C). HRMS (ESI) m/z for $\mathrm{C}_{27} \mathrm{H}_{34} \mathrm{O}_{3} \mathrm{~N}_{2} \mathrm{NaS}_{2}[\mathrm{M}+\mathrm{Na}]^{+}$calcd 521.19031, found 521.18896.

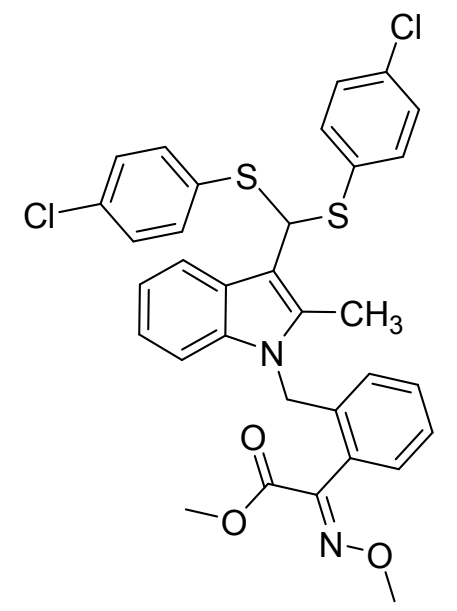

(E)-methyl-2-(2-((3-(bis((4-chlorophenyl)thio)methyl)-2-met hyl-1H-indol-1-yl)methyl)phenyl)-2-(methoxyimino) acetate (D25): Yield: $86 \%$; white solid; m. p. 97 - $99{ }^{\circ} \mathrm{C} ;{ }^{1} \mathrm{H}$ NMR $\left(500 \mathrm{MHz}, \mathrm{CDCl}_{3}\right) \delta 8.05(\mathrm{~d}, J=7.0 \mathrm{~Hz}, 1 \mathrm{H}, \mathrm{Ar}-\mathrm{H}), 7.31(\mathrm{~d}, J$ $=7.5 \mathrm{~Hz}, 1 \mathrm{H}, \mathrm{Ar}-\mathrm{H}), 7.29(\mathrm{~d}, J=1.9 \mathrm{~Hz}, 2 \mathrm{H}, \mathrm{Ar}-\mathrm{H}), 7.28(\mathrm{~d}, J$ $=2.0 \mathrm{~Hz}, 2 \mathrm{H}, \mathrm{Ar}-\mathrm{H}), 7.21(\mathrm{~d}, J=7.1 \mathrm{~Hz}, 1 \mathrm{H}, \mathrm{Ar}-\mathrm{H}), 7.18(\mathrm{t}, J=$ $2.0 \mathrm{~Hz}, 3 \mathrm{H}, \mathrm{Ar}-\mathrm{H}), 7.16$ (d, $J=1.3 \mathrm{~Hz}, 3 \mathrm{H}, \mathrm{Ar}-\mathrm{H}), 7.15$ (t, $J=$ $1.4 \mathrm{~Hz}, 1 \mathrm{H}, \mathrm{Ar}-\mathrm{H}), 7.09$ (d, $J=7.2 \mathrm{~Hz}, 1 \mathrm{H}, \mathrm{Ar}-\mathrm{H}), 6.05$ (d, $J=$ $7.9 \mathrm{~Hz}, 1 \mathrm{H}, \mathrm{Ar}-\mathrm{H}), 5.72$ (s, 1H, -SCHS-), 5.01 (s, 2H, - $\left.\mathrm{NCH}_{2}-\right), 4.15$ (s, 3H, - $\left.\mathrm{OCH}_{3}\right), 3.93(\mathrm{~s}$, $\left.3 \mathrm{H},-\mathrm{OCH}_{3}\right), 1.94\left(\mathrm{~s}, 3 \mathrm{H},-\mathrm{CH}_{3}\right) .{ }^{13} \mathrm{C} \mathrm{NMR}\left(100 \mathrm{MHz}, \mathrm{CDCl}_{3}\right) \delta 163.32(\mathrm{~s}, 1 \mathrm{C}), 149.18(\mathrm{~s}$, 1C), 136.70 (s, 1C), 135.43 (s, 1C), 134.45 (s, 4C), 134.08 (s, 2C), 133.53 (s, 2C), 130.06 (s, 
1C), $128.90(\mathrm{~s}, 4 \mathrm{C}), 128.36(\mathrm{~s}, 1 \mathrm{C}), 128.07$ (s, 1C), 127.27 (s, 1C), 125.76 (s, 1C), 125.07 (s, 1C), 121.87 (s, 1C), 120.32 (s, 1C), 119.89 (s, 1C), 109.32 (s, 1C), 109.07 (s, 1C), 64.08 (s, 1C), 54.21 (s, 1C), 53.30 (s, 1C), 44.16 (s, 1C), 31.63 (s, 1C), 22.70 (s, 1C), 14.18 (s, 1C), 10.06 (s, 1C). HRMS (ESI) $\mathrm{m} / \mathrm{z}$ for $\mathrm{C}_{32} \mathrm{H}_{28} \mathrm{O}_{3} \mathrm{~N}_{2} \mathrm{Cl}_{2} \mathrm{NaS}_{2}[\mathrm{M}+\mathrm{Na}]^{+}$calcd 657.08106, found 657.07947.<smiles>CO/N=C(/C(=O)OC)c1ccccc1Cn1c(Cl)c(C2SCCS2)c2ccccc21</smiles>

(E)-methyl-2-(2-((2-chloro-3-(1,3-dithiolan-2-yl)-1H-indol-1-yl)methyl )phenyl)-2-(methoxyimino)acetate (D26): Yield: $94 \%$; white solid; m. p. $124.1-124.8^{\circ} \mathrm{C} ;{ }^{1} \mathrm{H}$ NMR (400 MHz, DMSO- $\left.d_{6}\right) \delta 7.95(\mathrm{~d}, J=8.1 \mathrm{~Hz}$, 1H, Ar-H), 7.32 (t, $J=6.0 \mathrm{~Hz}, 2 \mathrm{H}, \mathrm{Ar}-\mathrm{H}), 7.24$ (d, $J=6.6 \mathrm{~Hz}, 1 \mathrm{H}, \mathrm{Ar}-\mathrm{H})$, 7.22 - 7.14 (m, 2H, Ar-H), 7.12 (d, J=7.8 Hz, 1H, Ar-H), 6.56 (d, $J=7.4$ $\mathrm{Hz}, 1 \mathrm{H}, \mathrm{Ar}-\mathrm{H}), 6.10$ (s, 1H, -SCHS-), 5.23 (s, 2H, - $\left.\mathrm{NCH}_{2}-\right), 4.04$ (s, 3H, $\left.-\mathrm{OCH}_{3}\right), 3.76\left(\mathrm{~s}, 3 \mathrm{H},-\mathrm{OCH}_{3}\right), 3.70-3.59\left(\mathrm{~m}, 2 \mathrm{H},-\mathrm{SCH}_{2}-\right), 3.47-3.38$ (m, 2H, - $\left.\mathrm{SCH}_{2}-\right) .{ }^{13} \mathrm{C}$ NMR (100 MHz, DMSO- $\left.d_{6}\right) \delta 162.95$ (s, 1C), 148.86 (s, 1C), 136.04 (s, 1C), 135.44 (s, 1C), 130.15 (s, 1C), 129.38 (s, 1C), 129.01 (s, 1C), 127.80 (s, 1C), 125.89 (s, 1C), 124.73 (s, 1C), 124.62 (s, 1C), 122.98 (s, 1C), 120.75 (s, 1C), 120.46 (s, 1C), 110.80 (s, 1C), 107.44 (s, 1C), 64.00 (s, 1C), 53.19 (s, 1C), 48.32 (s, 1C), 45.14 (s, 1C), 40.51 (s, 1C), 40.51 (s, 2C). HRMS (ESI) m/z for $\mathrm{C}_{22} \mathrm{H}_{22} \mathrm{O}_{3} \mathrm{~N}_{2} \mathrm{ClS}_{2}[\mathrm{M}+\mathrm{H}]{ }^{+}$calcd 461.07549, found 461.07425 .<smiles>CO/N=C(/C(=O)OC)c1ccccc1Cn1c(Br)c(C2SCCS2)c2ccccc21</smiles>

(E)-methyl-2-(2-((2-bromo-3-(1,3-dithiolan-2-yl)-1H-indol-1-yl)methy I)phenyl)-2-(methoxyimino)acetate (D27): Yield: $92 \%$; white solid; m. p. $154.1-155.2{ }^{\circ} \mathrm{C} ;{ }^{1} \mathrm{H}$ NMR $\left(400 \mathrm{MHz}\right.$, DMSO- $\left.d_{6}\right) \delta 7.98(\mathrm{~d}, J=7.9 \mathrm{~Hz}$, 1H, Ar-H), 7.36 - 7.27 (m, 2H, Ar-H), .26 - 7.22 (m, 1H, Ar-H), 7.14 (d, J $=6.9 \mathrm{~Hz}, 2 \mathrm{H}, \mathrm{Ar}-\mathrm{H}), 7.11-7.04(\mathrm{~m}, 1 \mathrm{H}, \operatorname{Ar}-\mathrm{H}), 6.42(\mathrm{~d}, \mathrm{~J}=7.5 \mathrm{~Hz}, 1 \mathrm{H}$, Ar-H), 6.07 (s, 1H, -SCHS-), 5.25 (s, 2H, - $\left.\mathrm{NCH}_{2}-\right), 4.06$ (s, 3H, - $\left.\mathrm{OCH}_{3}\right)$, $3.78\left(\mathrm{~s}, 3 \mathrm{H},-\mathrm{OCH}_{3}\right), 3.70-3.62\left(\mathrm{~m}, 2 \mathrm{H},-\mathrm{SCH}_{2}-\right), 3.45(\mathrm{dd}, \mathrm{J}=7.1,3.8$

$\left.\mathrm{Hz}, 2 \mathrm{H},-\mathrm{SCH}_{2}-\right) .{ }^{13} \mathrm{C} \mathrm{NMR}\left(100 \mathrm{MHz}, \mathrm{DMSO}-d_{6}\right) \delta 163.01$ (s, 1C), 148.91 (s, 1C), 137.30 (s, 1C), $135.50(\mathrm{~s}, 1 \mathrm{C}), 130.14$ (s, 1C), 129.26 (s, 1C), 128.96 (s, 1C), 127.71 (s, 1C), 125.53 (s, 1C), 125.38 (s, 1C), 122.93 (s, 1C), 120.73 (s, 1C), 120.28 (s, 1C), 115.26 (s, 1C), 110.93 (s, 
1C), 110.52 (s, 1C), 64.03 (s, 2C), 53.22 (s, 2C), 49.88 (s, 1C), 46.44 (s, 1C). HRMS (ESI) $\mathrm{m} / \mathrm{z}$ for $\mathrm{C}_{22} \mathrm{H}_{21} \mathrm{O}_{3} \mathrm{~N}_{2} \mathrm{BrKS}_{2}[\mathrm{M}+\mathrm{K}]^{+}$calcd 542.98085, found 542.98016 .<smiles>CO/N=C(/C(=O)OC)c1ccccc1Cn1c(Cl)c(C2SCCS2)c2cc(Br)ccc21</smiles>

(E)-methyl-2-(2-((5-bromo-2-chloro-3-(1,3-dithiolan-2-yl)-1H-indol -1-yl)methyl)phenyl)-2-(methoxyimino)acetate (D28): Yield: $91 \%$; white solid; m. p. $171.5-172.3{ }^{\circ} \mathrm{C} ;{ }^{1} \mathrm{H}$ NMR (400 MHz, DMSO- $\left.d_{6}\right) \delta$ $8.07(\mathrm{~d}, J=1.9 \mathrm{~Hz}, 1 \mathrm{H}, \mathrm{Ar}-\mathrm{H}), 7.36-7.33(\mathrm{~m}, 1 \mathrm{H}, \mathrm{Ar}-\mathrm{H}), 7.33$ (d, $J=$ $3.5 \mathrm{~Hz}, 1 \mathrm{H}, \mathrm{Ar}-\mathrm{H}), 7.31$ (d, J=2.5 Hz, 1H, Ar-H), 7.26 - 7.21 (m, 1H, Ar-H), 7.18 (d, $J=8.8 \mathrm{~Hz}, 1 \mathrm{H}, \mathrm{Ar}-\mathrm{H}), 6.61$ (d, $J=7.7 \mathrm{~Hz}, 1 \mathrm{H}, \mathrm{Ar}-\mathrm{H})$, 6.06 (s, 1H, -SCHS-), 5.24 (s, 2H, - $\left.\mathrm{NCH}_{2}-\right), 4.02\left(\mathrm{~s}, 3 \mathrm{H},-\mathrm{OCH}_{3}\right), 3.73$ $\left(\mathrm{s}, 3 \mathrm{H},-\mathrm{OCH}_{3}\right), 3.68$ - $3.62\left(\mathrm{~m}, 2 \mathrm{H},-\mathrm{SCH}_{2}-\right), 3.45-3.41\left(\mathrm{~m}, 2 \mathrm{H},-\mathrm{SCH}_{2}-\right) .{ }^{13} \mathrm{C} \mathrm{NMR}(100$ MHz, DMSO-d $\left.d_{6}\right) \delta 162.84$ (s, 1C), 148.78 (s, 1C), 135.06 (s, 1C), 134.85 (s, 1C), 130.19 (s, 1C), 129.47 (s, 1C), 129.11 (s, 1C), 127.94 (s, 1C), 126.30 (s, 2C), 126.11 (s, 1C), 125.53 (s, 1C), 122.63 (s, 1C), 113.17 (s, 1C), 113.11 (s, 1C), 106.99 (s, 1C), 64.00 (s, 1C), 53.16 (s, 1C), 48.14 (s, 1C), 45.57 (s, 1C), 40.48 (s, 2C). HRMS (ESI) m/z for $\mathrm{C}_{22} \mathrm{H}_{20} \mathrm{O}_{3} \mathrm{~N}_{2} \mathrm{BrClNaS}_{2}$ $[\mathrm{M}+\mathrm{Na}]^{+}$calcd 560.96795 , found 560.96655 . 


\section{2. ${ }^{1} \mathrm{H},{ }^{13} \mathrm{C}$ NMR, HRMS data}

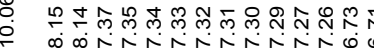

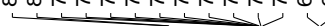

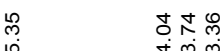<smiles>O=Cc1c(Cl)[nH]c2ccccc12</smiles><smiles>CCc1ccccc1/C(=N\OC)C(=O)OC</smiles>

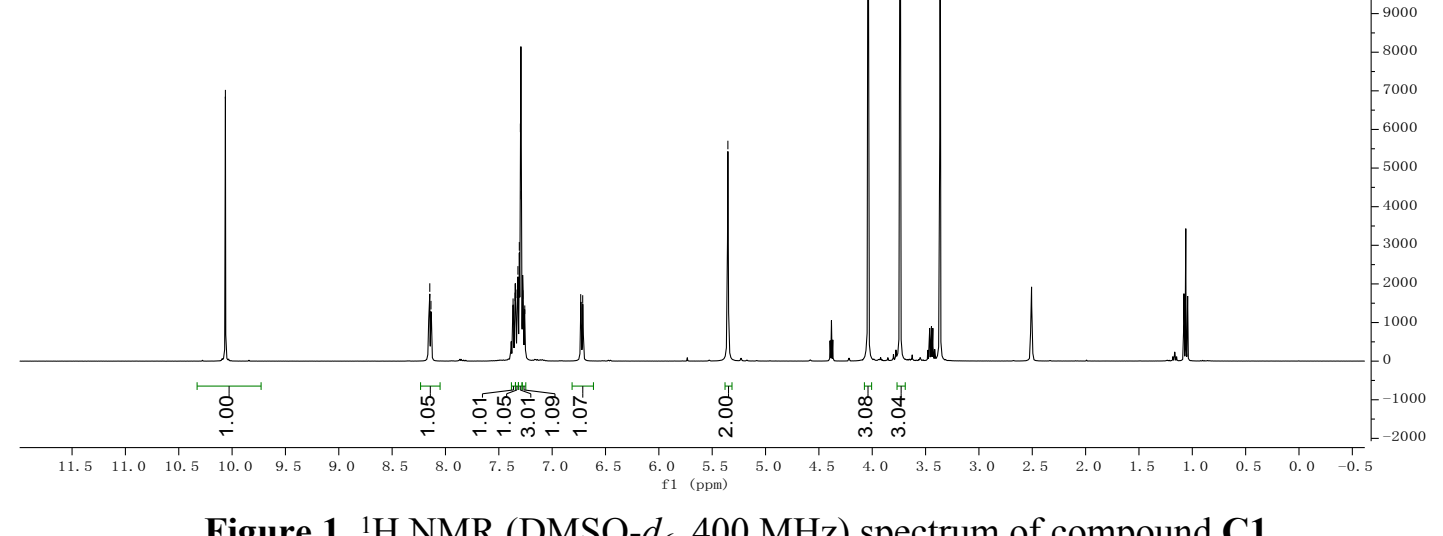

Figure 1. ${ }^{1} \mathrm{H}$ NMR (DMSO- $d_{6}, 400 \mathrm{MHz}$ ) spectrum of compound $\mathbf{C 1}$. 


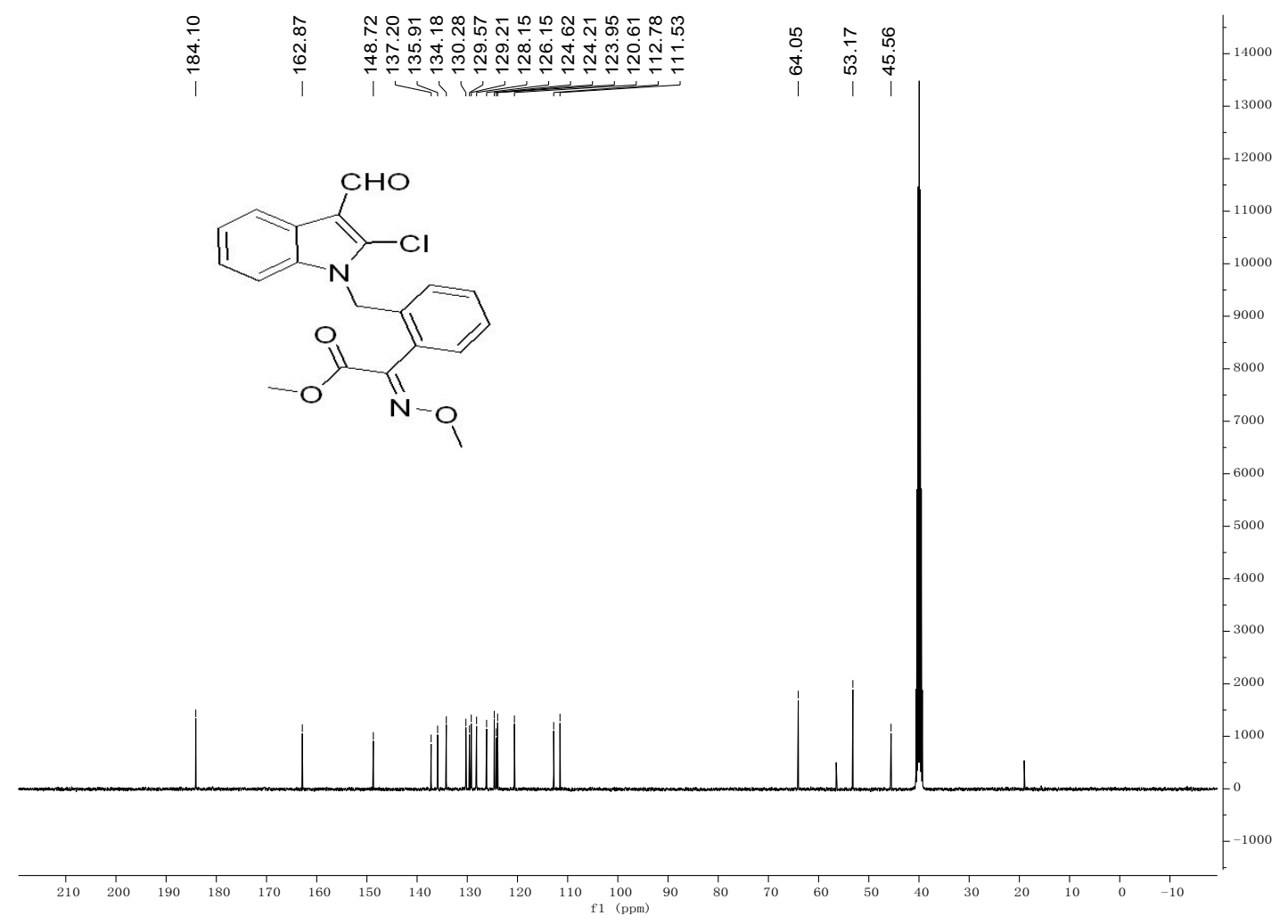

Figure 2. ${ }^{13} \mathrm{C}$ NMR (DMSO- $d_{6}, 400 \mathrm{MHz}$ ) spectrum of compound $\mathbf{C 1}$.

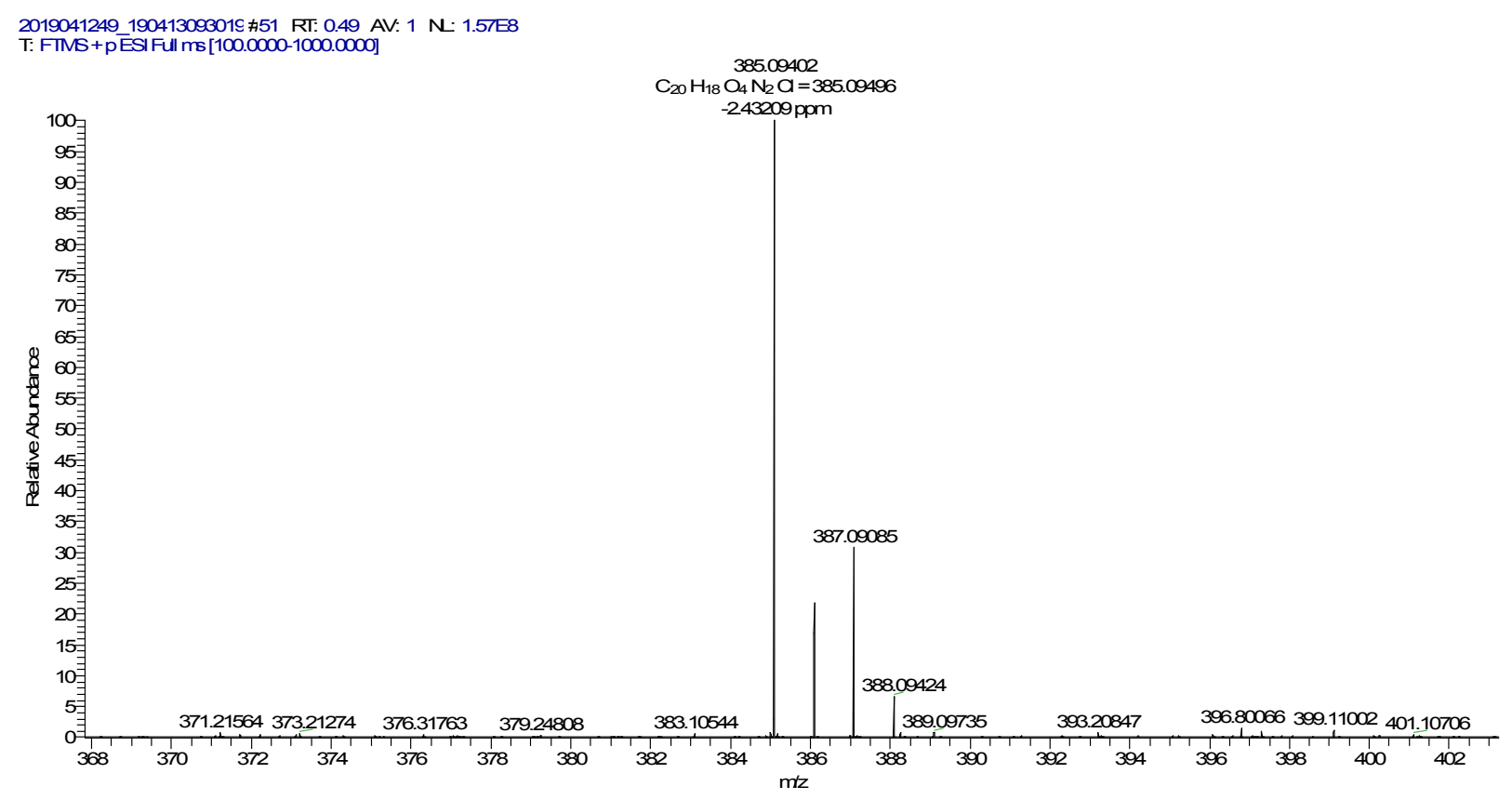

Figure 3. HRMS of compound C1. 


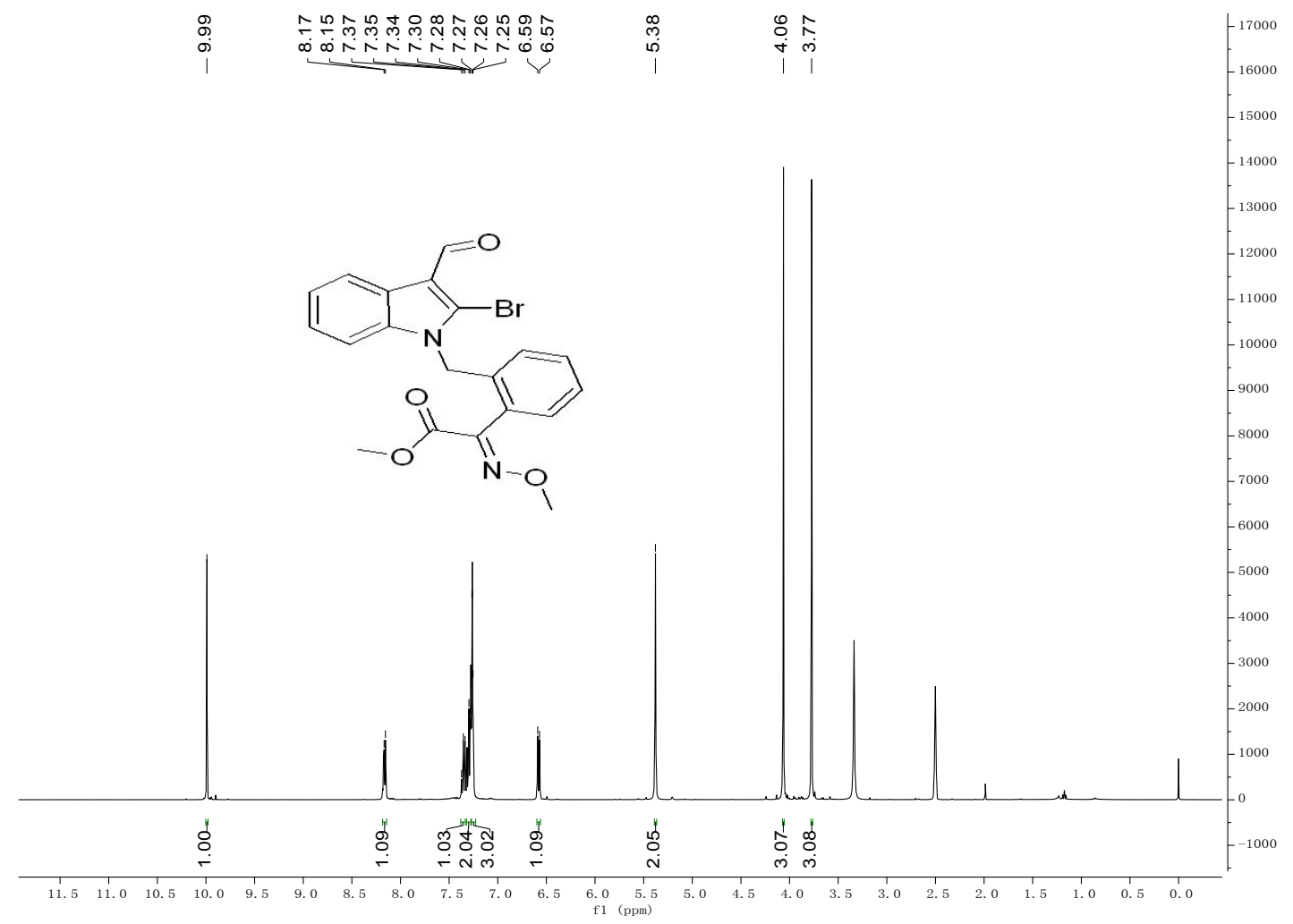

Figure 4. ${ }^{1} \mathrm{H}$ NMR (DMSO- $d_{6}, 400 \mathrm{MHz}$ ) spectrum of compound $\mathbf{C 2}$.

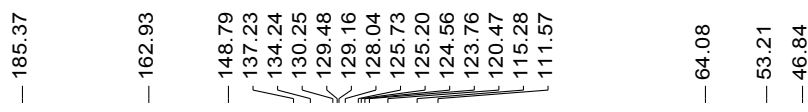<smiles>CO/N=C(/C(=O)OC)c1ccccc1Cn1c(Br)c(C=O)c2ccccc21</smiles>

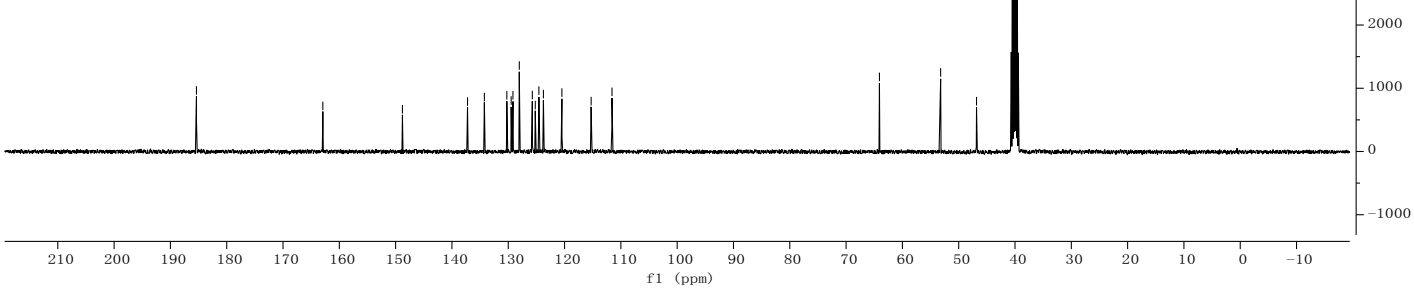

Figure 5. ${ }^{13} \mathrm{C}$ NMR (DMSO- $d_{6}, 400 \mathrm{MHz}$ ) spectrum of compound $\mathbf{C} 2$. 


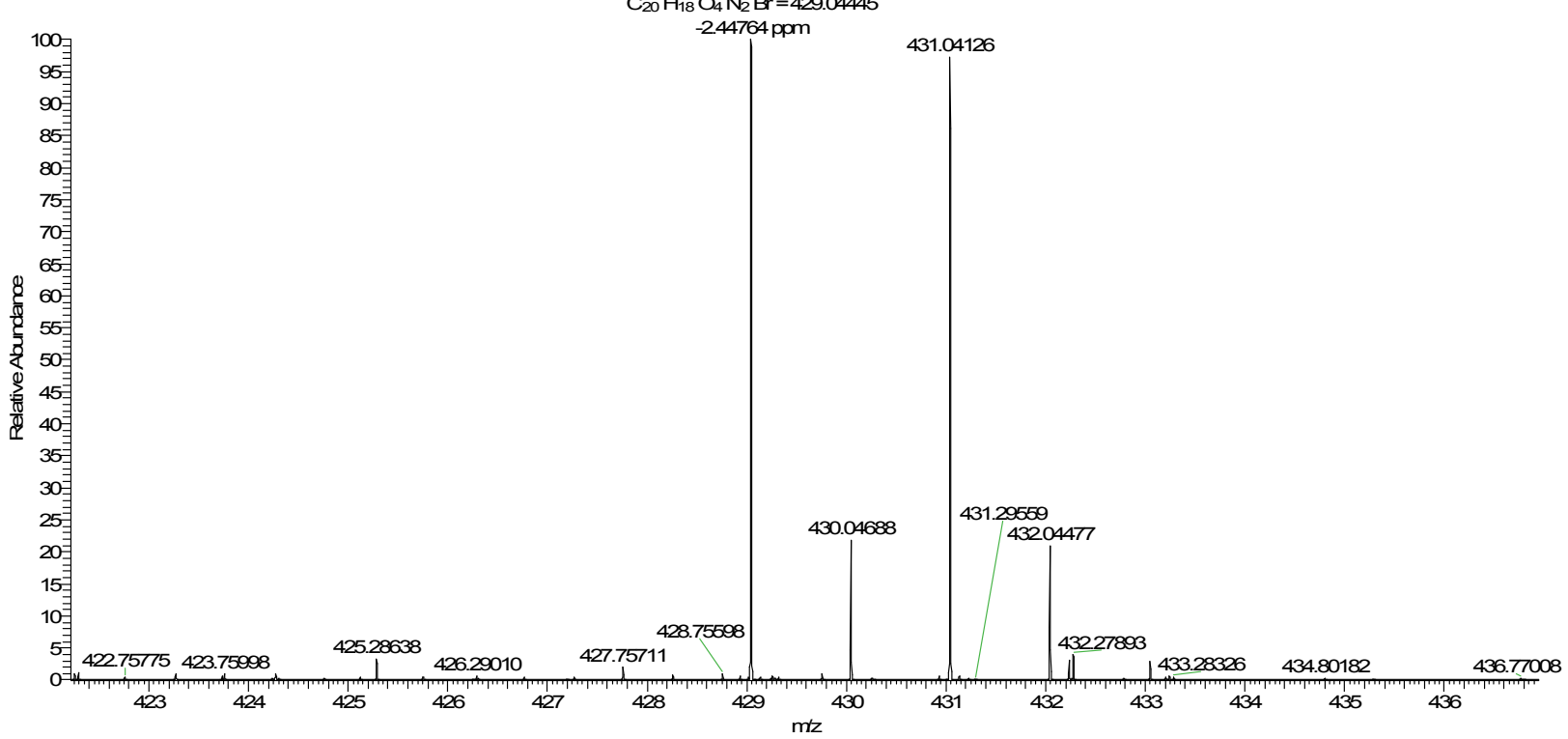

Figure 6. HRMS of compound C2.

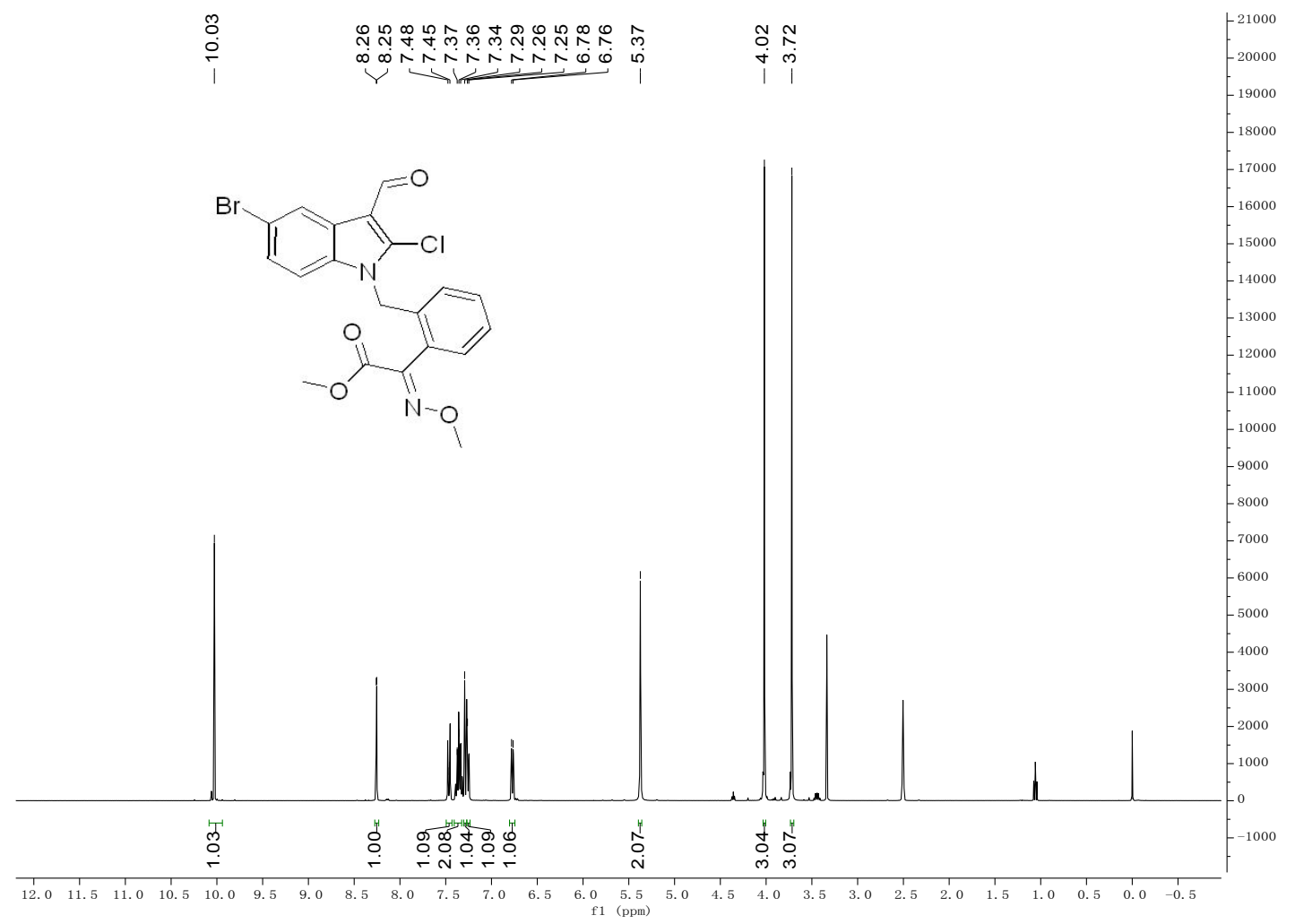

Figure 7. ${ }^{1} \mathrm{H}$ NMR (DMSO- $d_{6}, 400 \mathrm{MHz}$ ) spectrum of compound $\mathbf{C 3}$. 


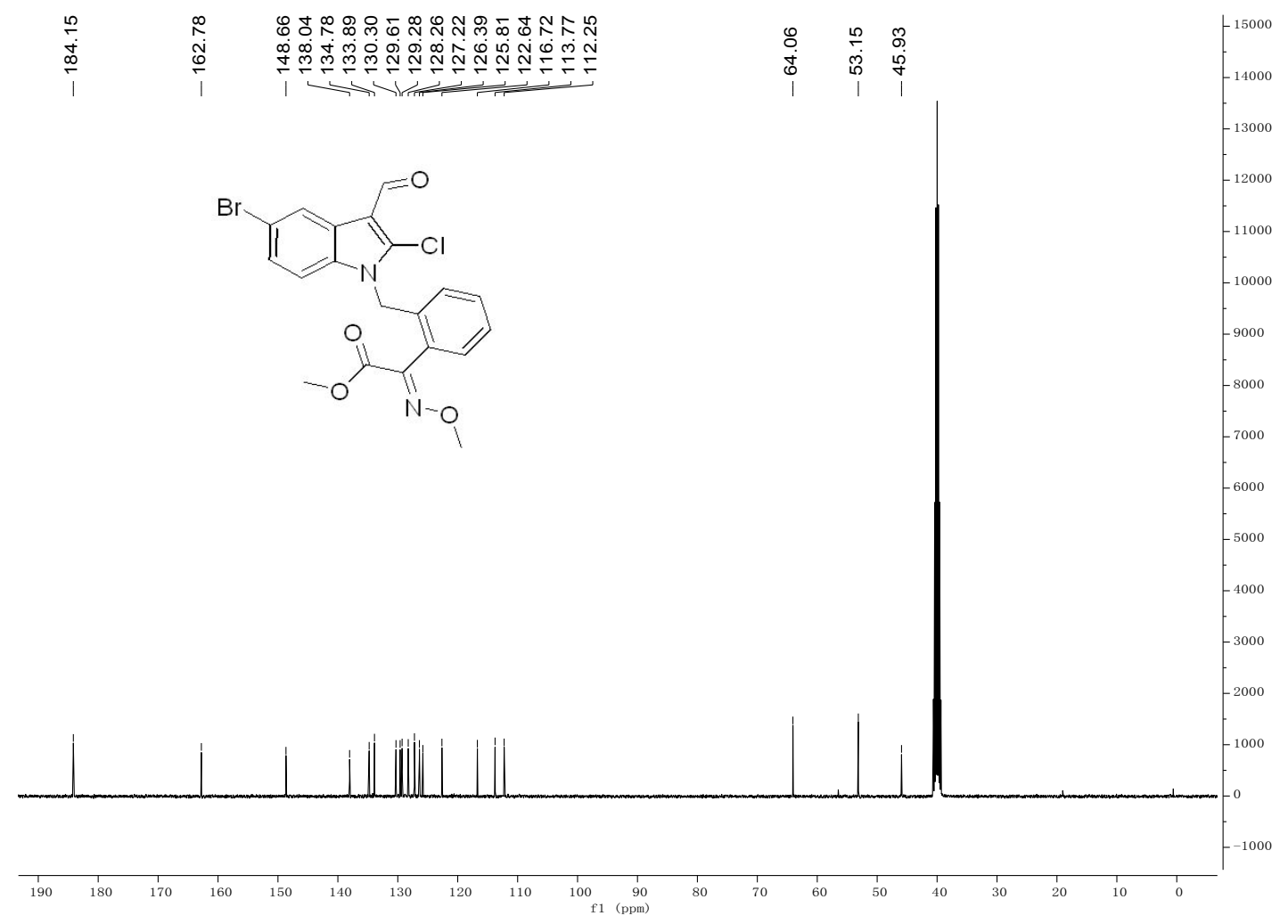

Figure 8. ${ }^{13} \mathrm{C}$ NMR $\left(\mathrm{CDCl}_{3}, 125 \mathrm{MHz}\right)$ spectrum of compound $\mathbf{C 3}$.

\section{4 \#57 Rा: 0.55 AV: 1 N.211E8}

T: FIMS + p ESI Full ms [100.0000-1000.0000]

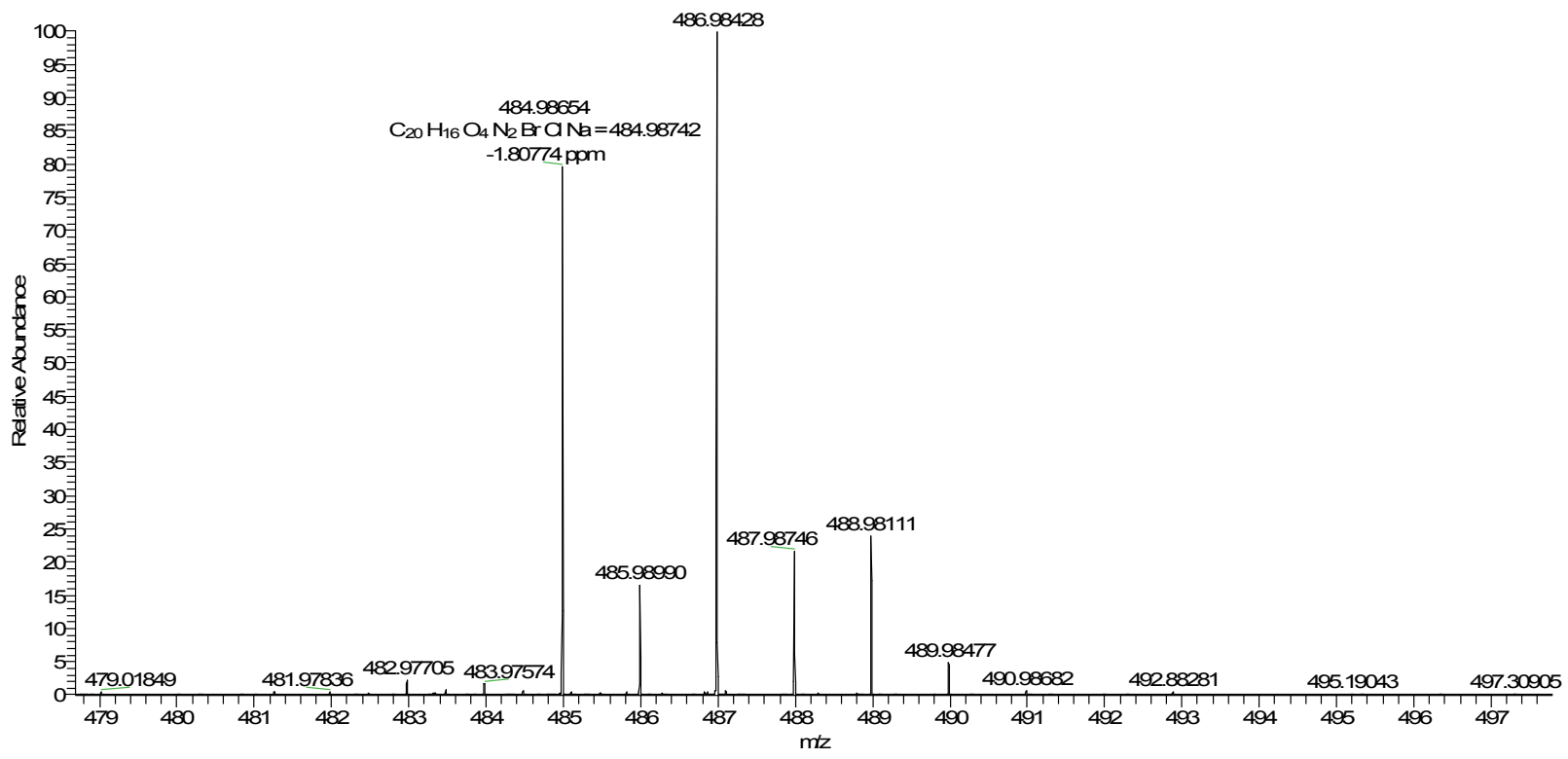

Figure 9. HRMS of compound C3. 


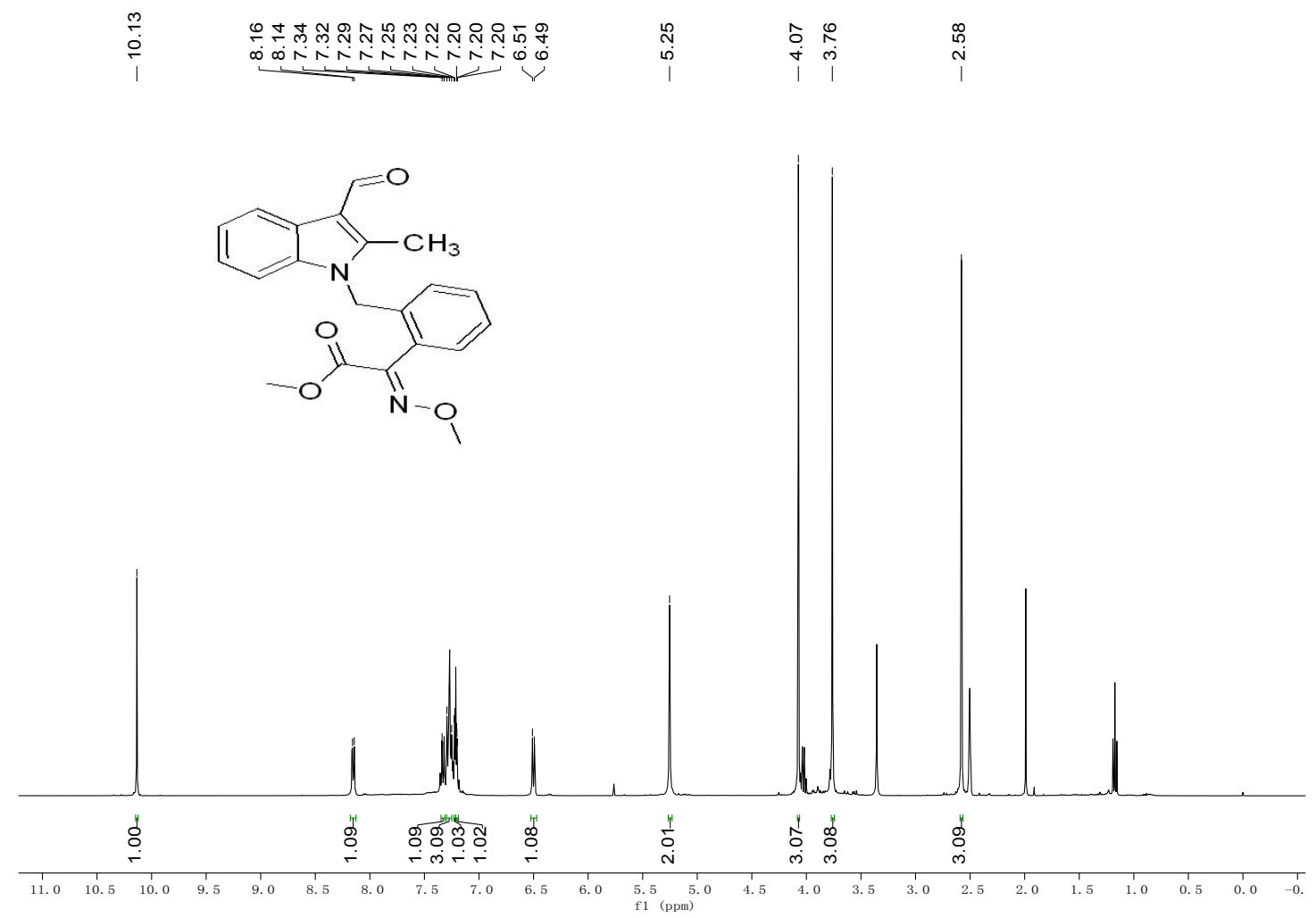

Figure 10. ${ }^{1} \mathrm{H}$ NMR (DMSO- $d_{6}, 400 \mathrm{MHz}$ ) spectrum of compound $\mathbf{C 4}$.
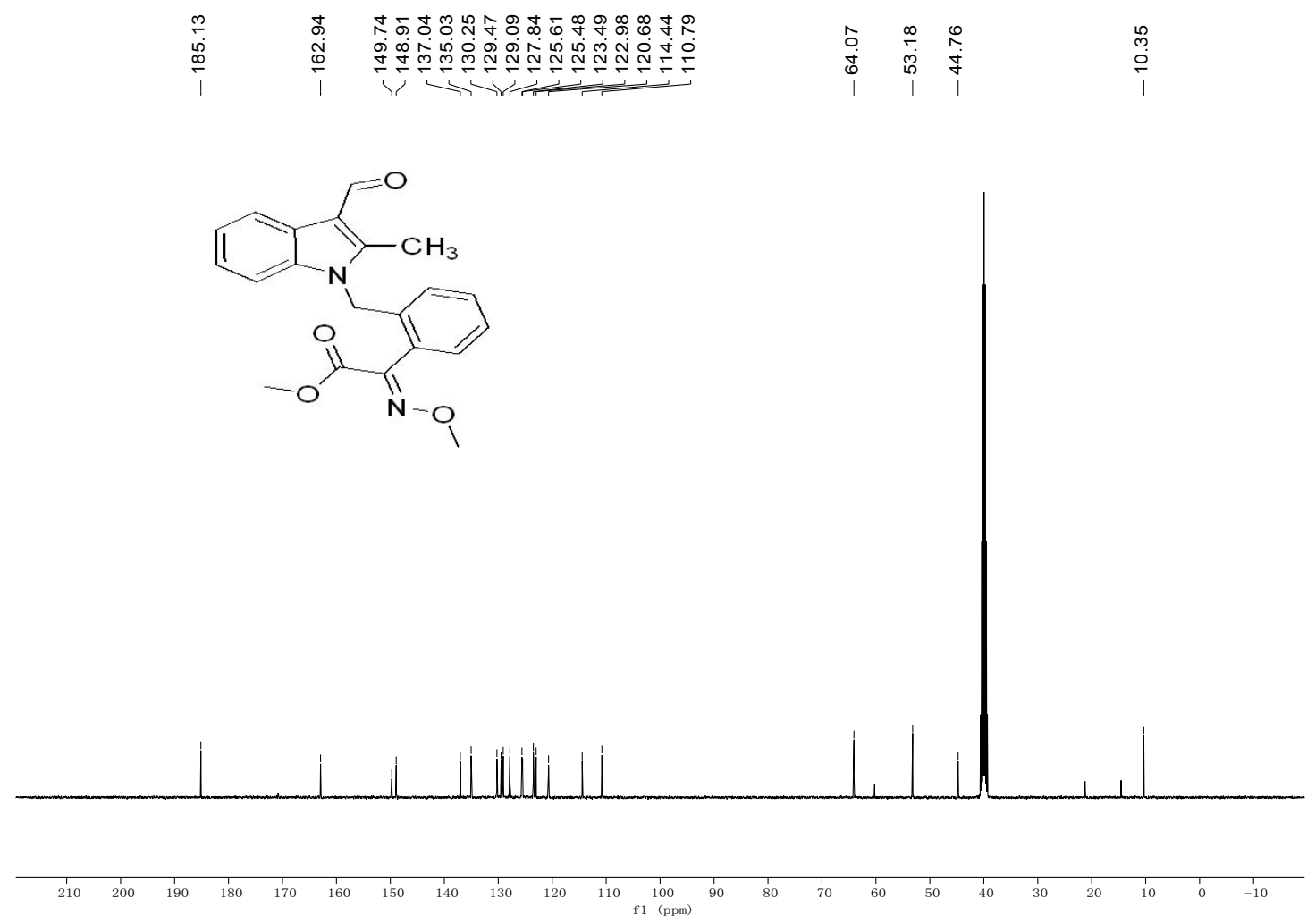

Figure 11. ${ }^{13} \mathrm{C} \mathrm{NMR}\left(\mathrm{CDCl}_{3}, 100 \mathrm{MHz}\right)$ spectrum of compound $\mathbf{C 4}$. 


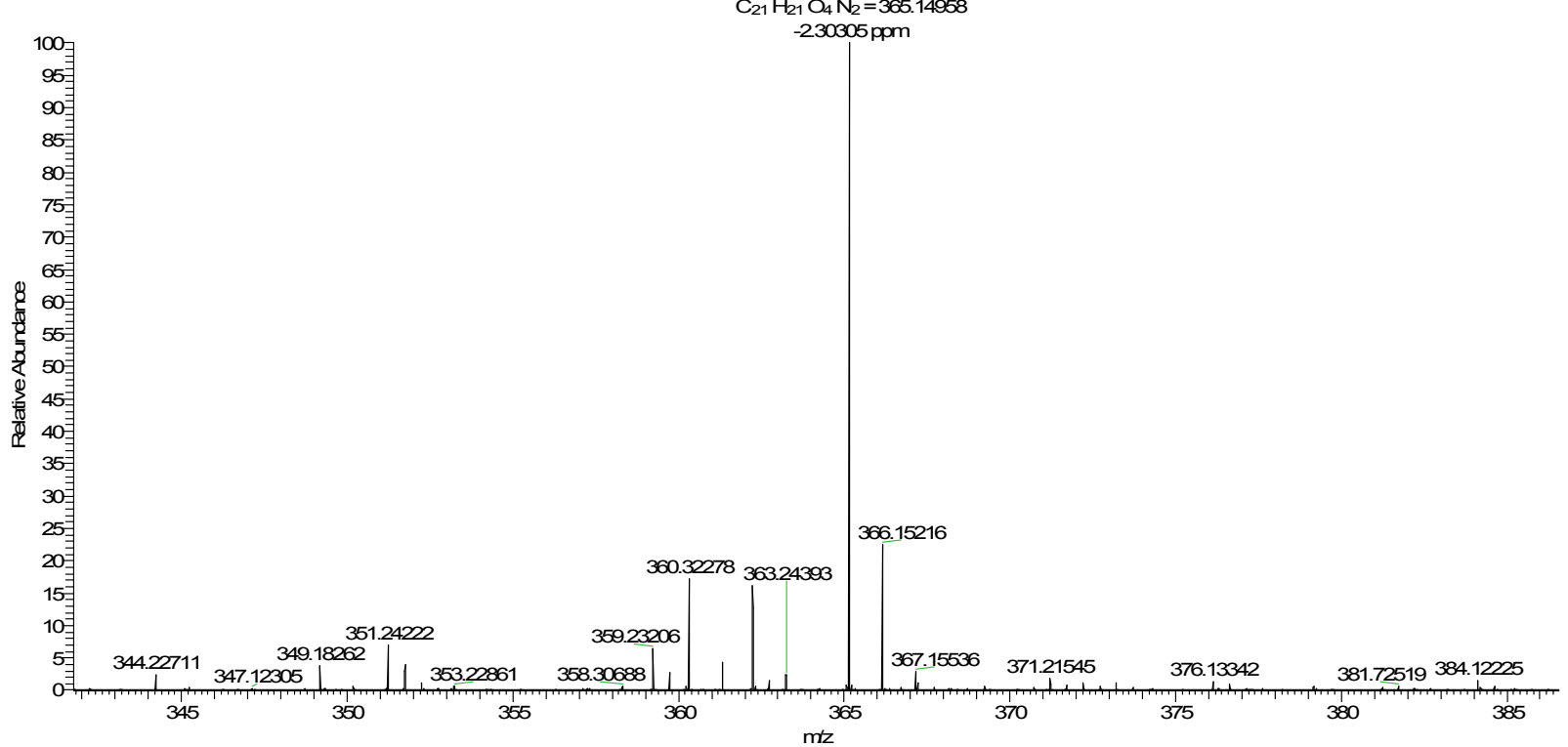

Figure 12. HRMS of compound C4.

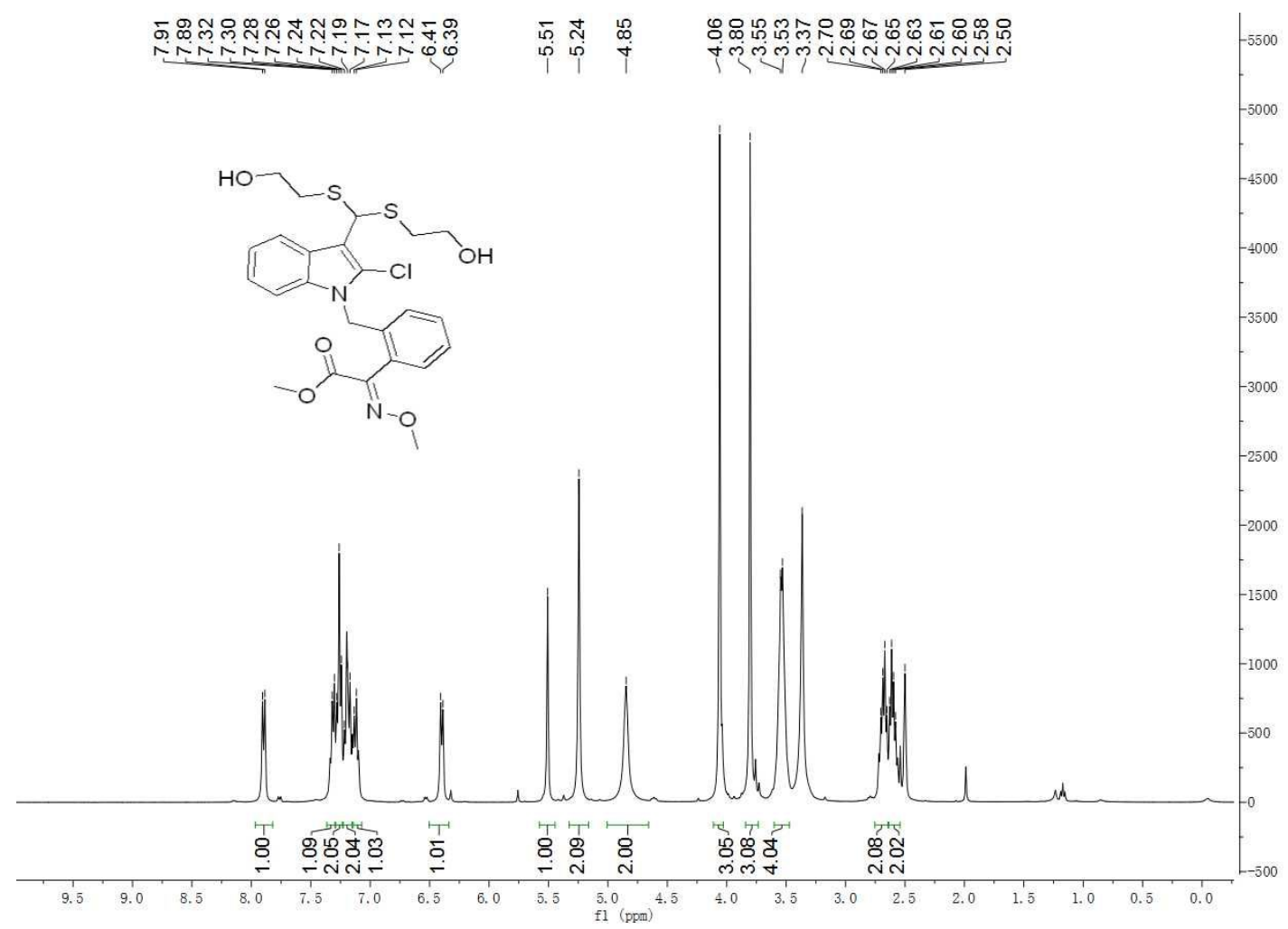

Figure 13. ${ }^{1} \mathrm{H}$ NMR (400 MHz, DMSO $d_{6}$ ) spectrum of compound D1. 


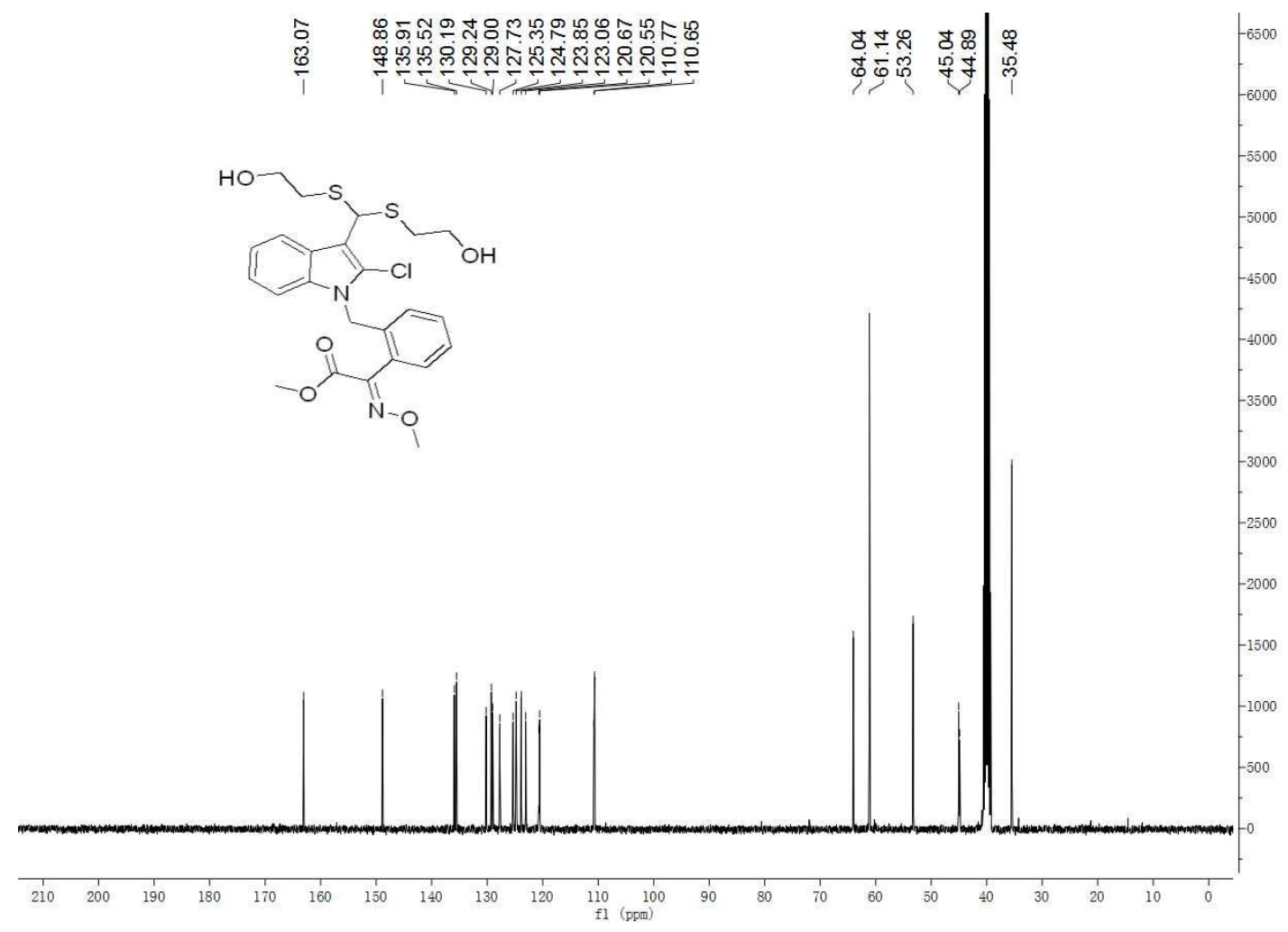

Figure 14. ${ }^{13} \mathrm{C}$ NMR (100 MHz, DMSO- $d_{6}$ ) spectrum of compound D1.

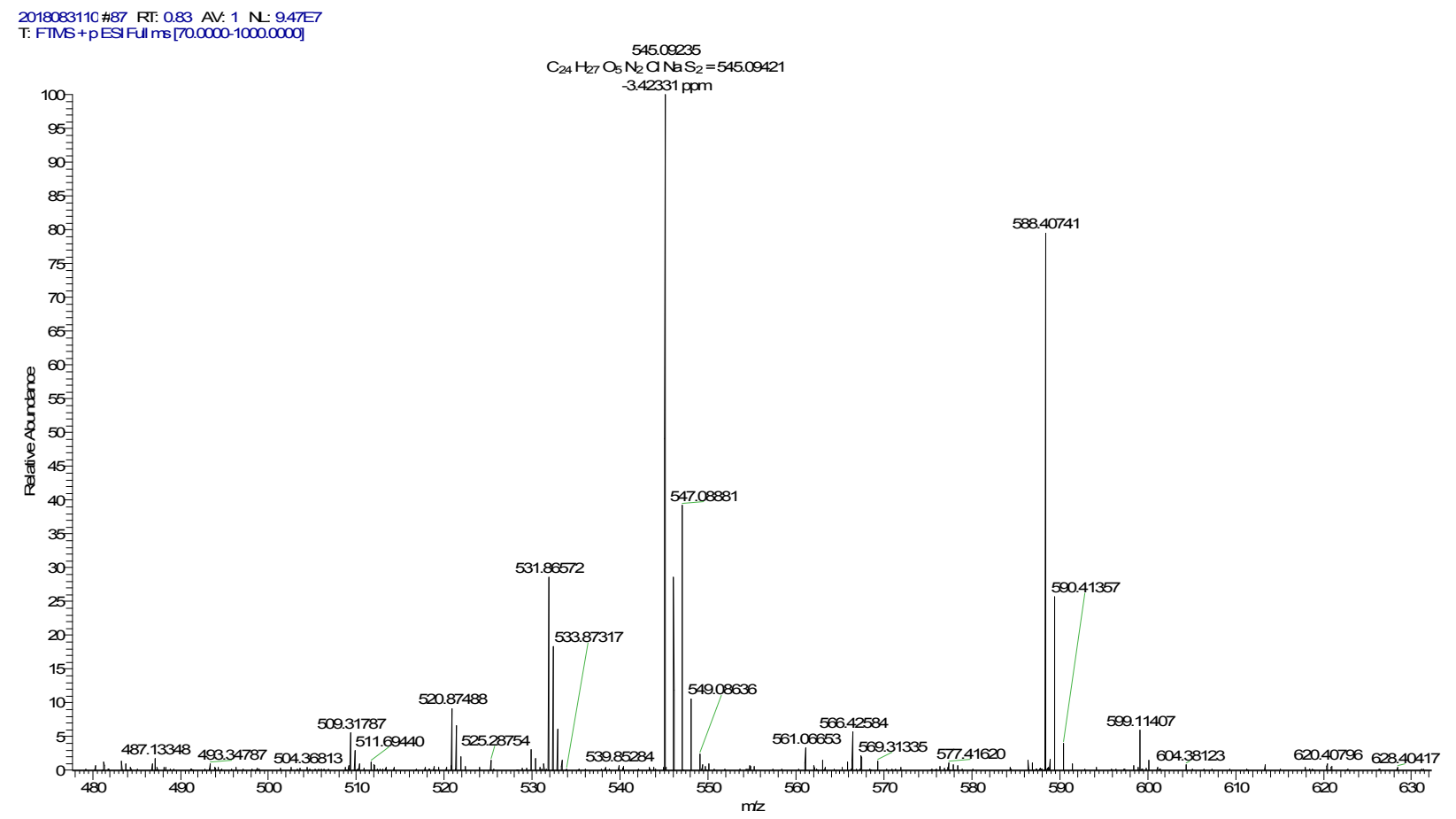

Figure 15. HRMS of compound D1. 


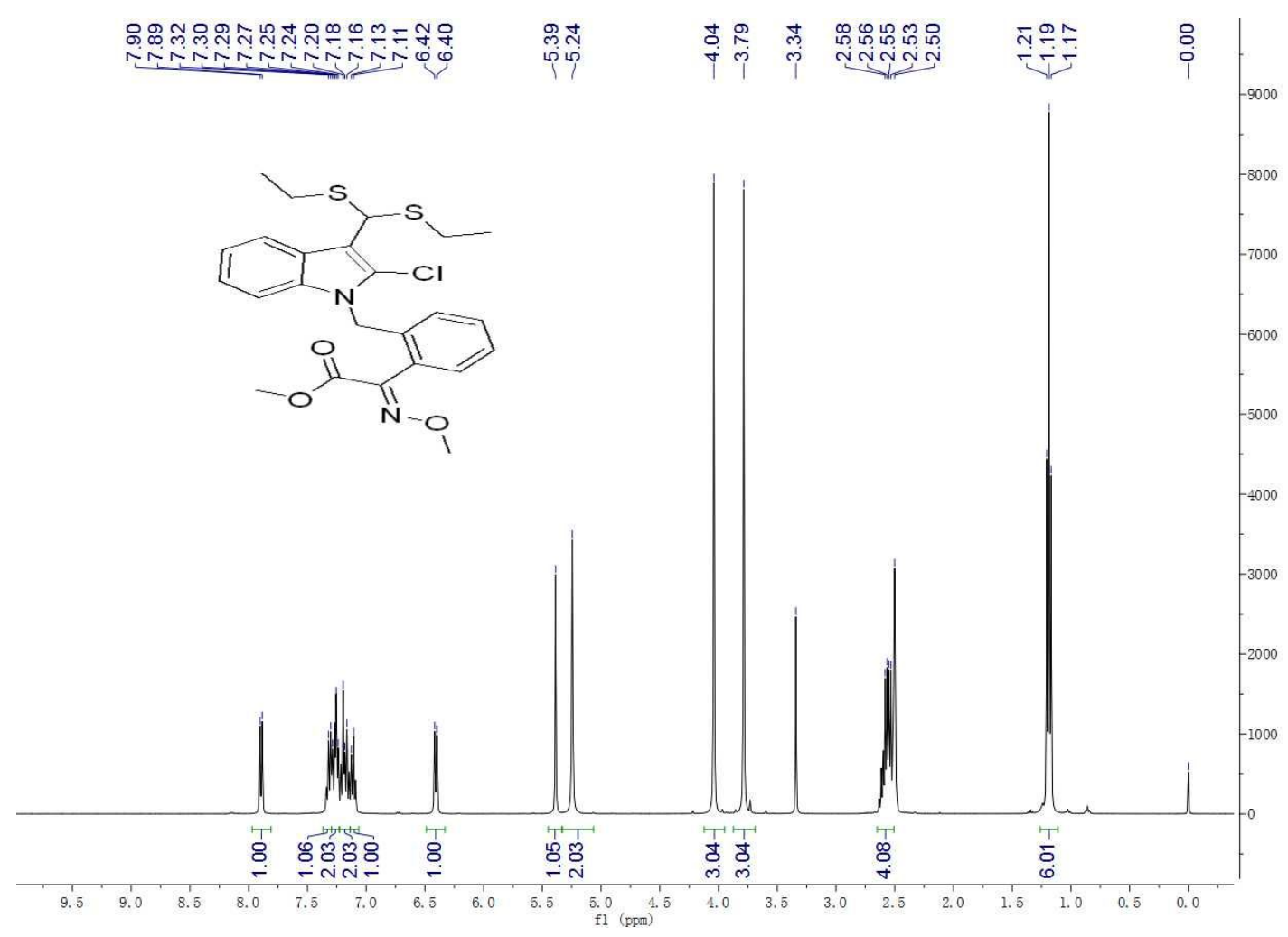

Figure 16. ${ }^{1} \mathrm{H}$ NMR ((400 MHz, DMSO- $\left.d_{6}\right)$ spectrum of compound D2.
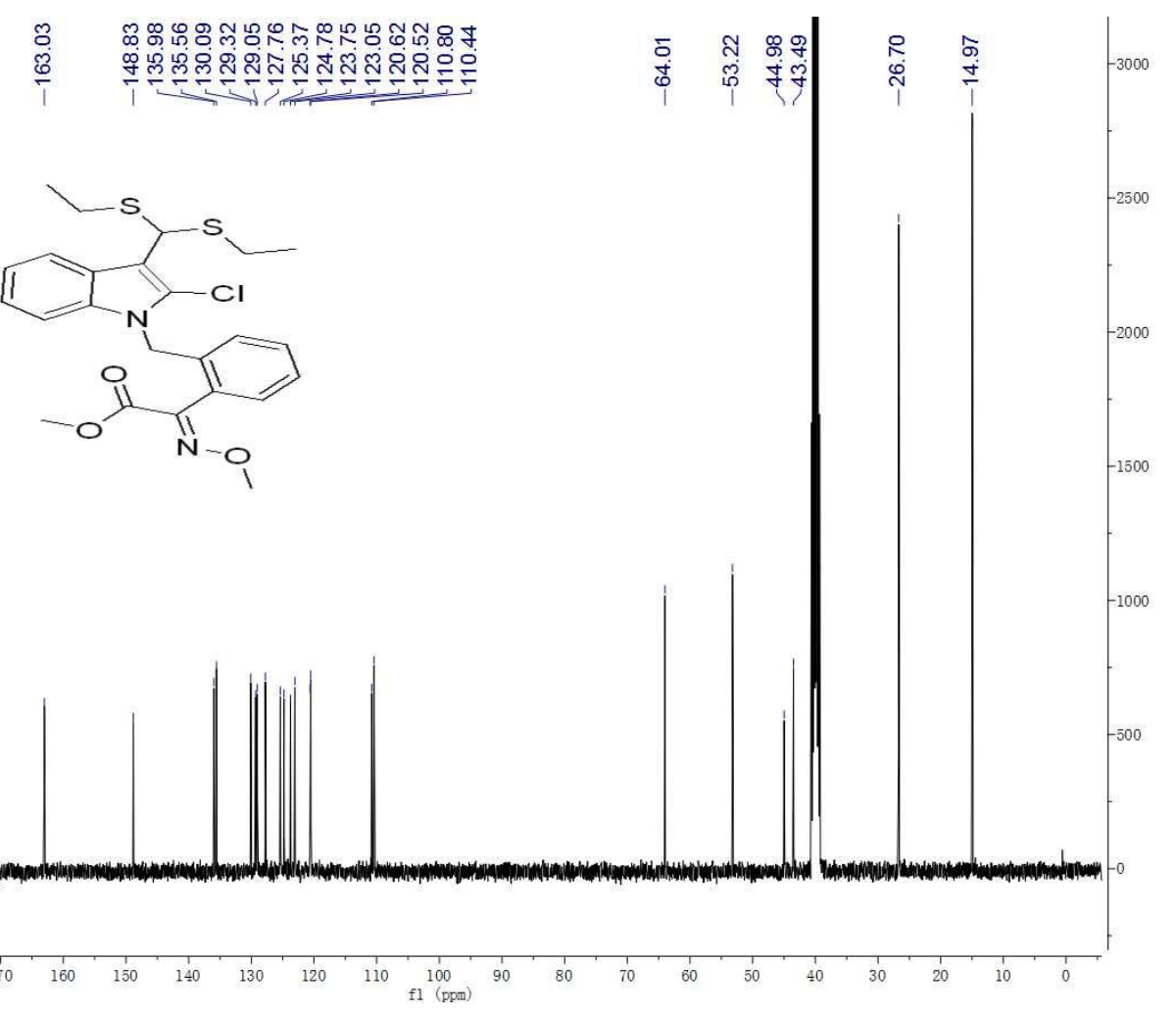

Figure 17. ${ }^{13} \mathrm{C}$ NMR $\left(100 \mathrm{MHz}, \mathrm{DMSO}-d_{6}\right)$ spectrum of compound D2. 
2018083118 \#209 Rा: 1.99 AV: 1 N.2 $240 E$

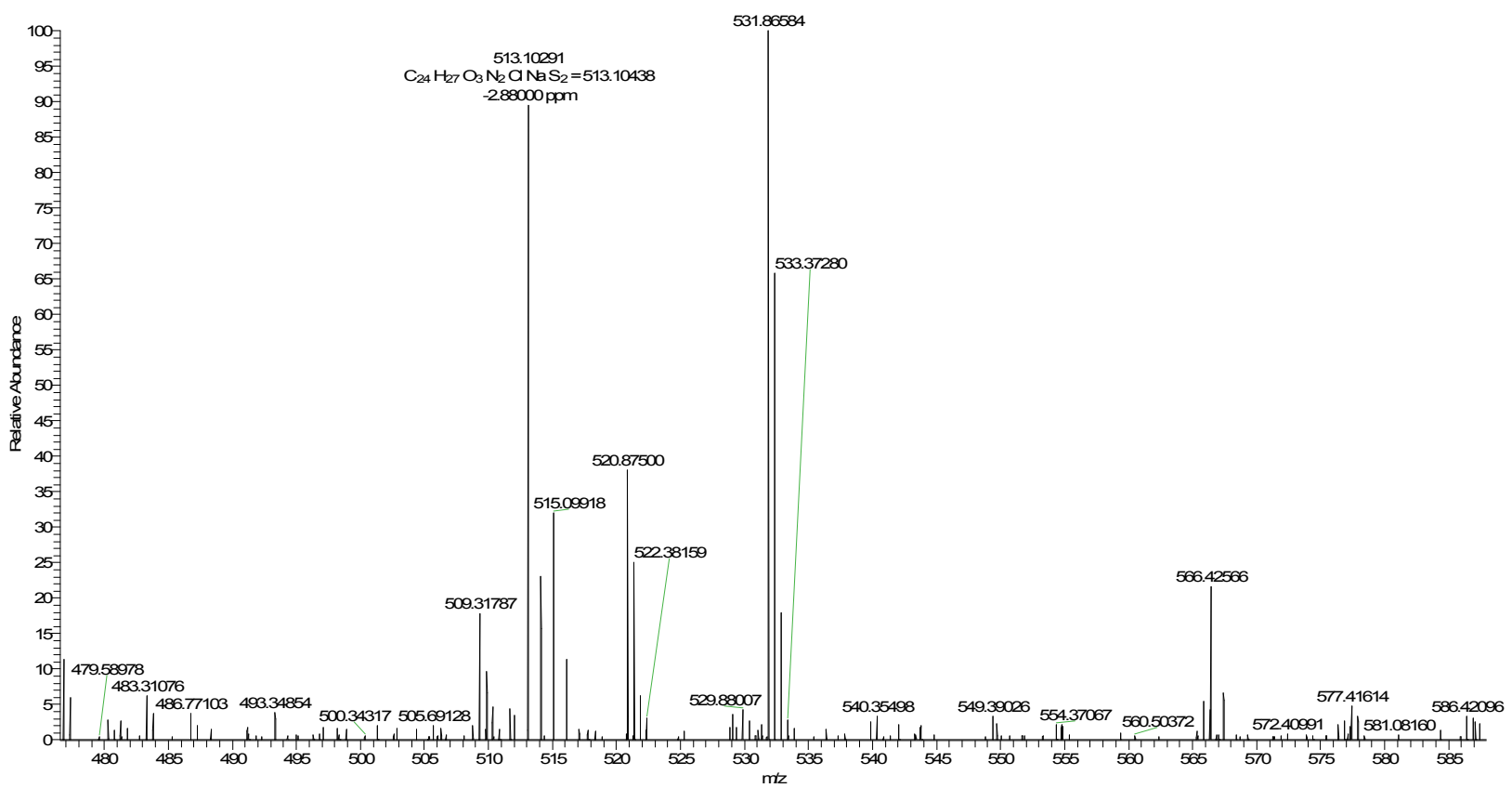

Figure 18. HRMS of compound D2.

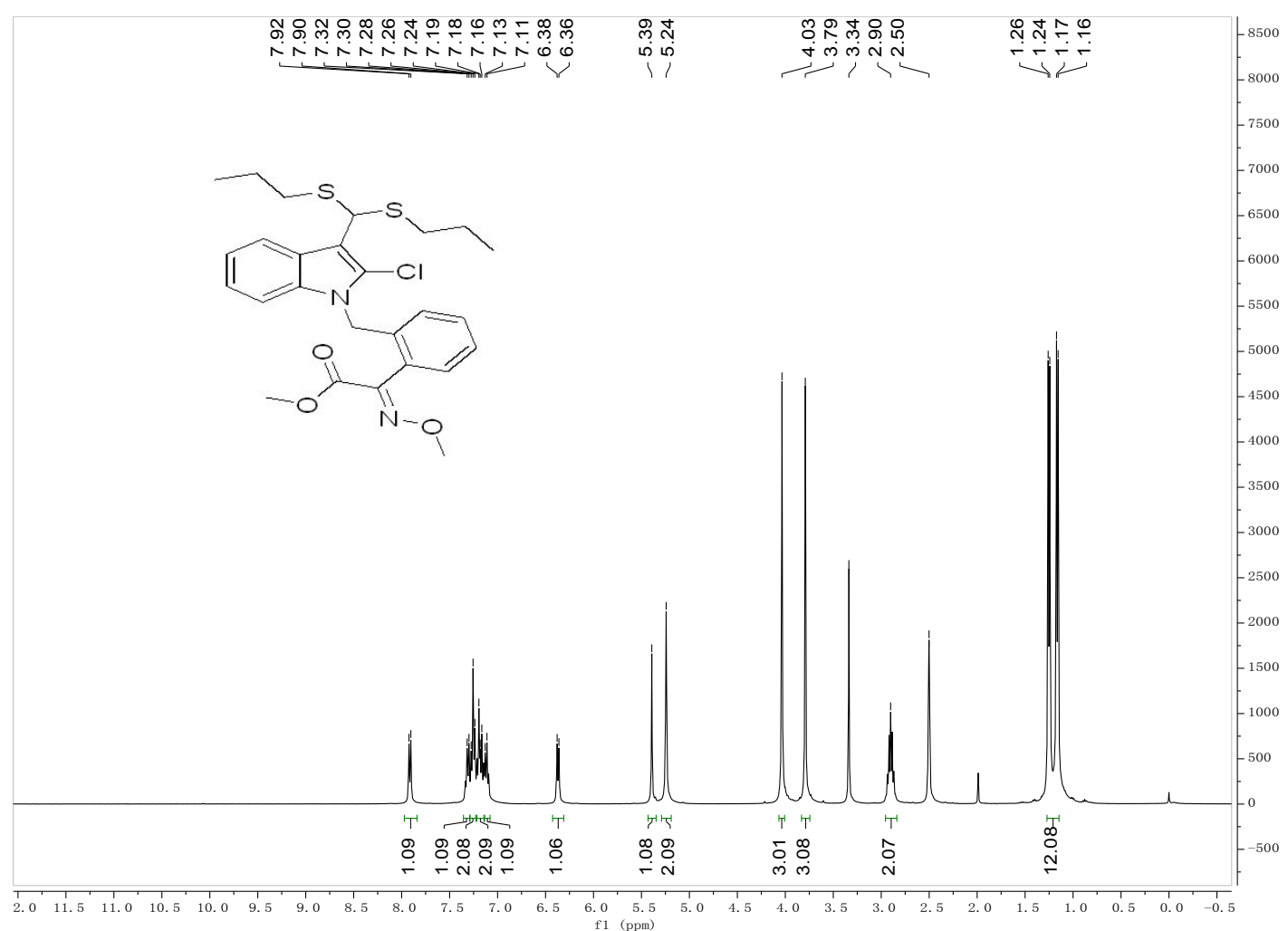

Figure 19. ${ }^{1} \mathrm{H}$ NMR $\left(\left(400 \mathrm{MHz}, \mathrm{DMSO}-d_{6}\right)\right.$ spectrum of compound D3. 


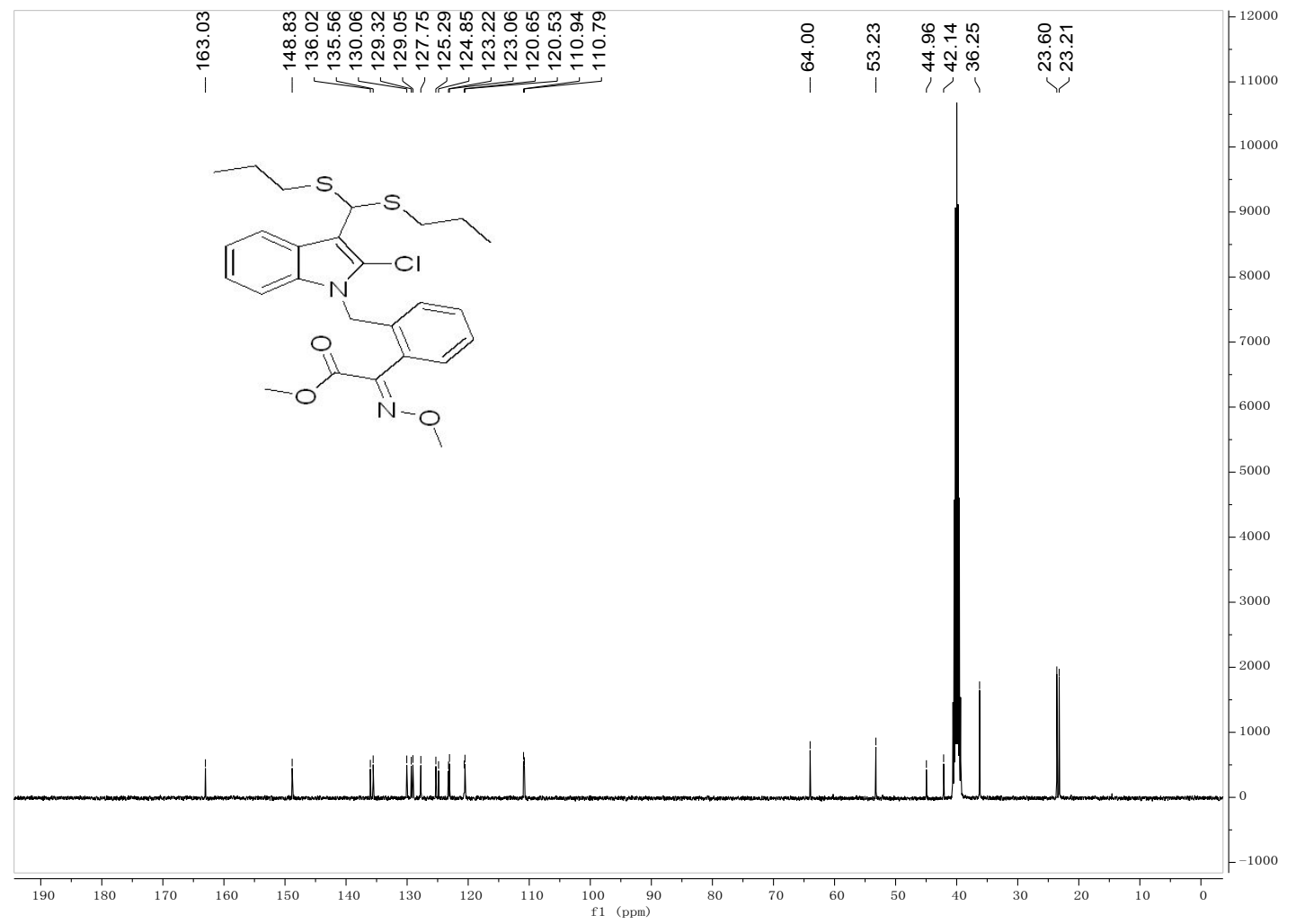

Figure 20. ${ }^{13} \mathrm{C}$ NMR $\left(100 \mathrm{MHz}\right.$, DMSO- $\left.d_{6}\right)$ spectrum of compound D3.

2019041254 \#77 Rा: 0.74 AV: 1 N- 6.75E/ T: FIMS + pESI Full ms [100.0000-1000.0000]

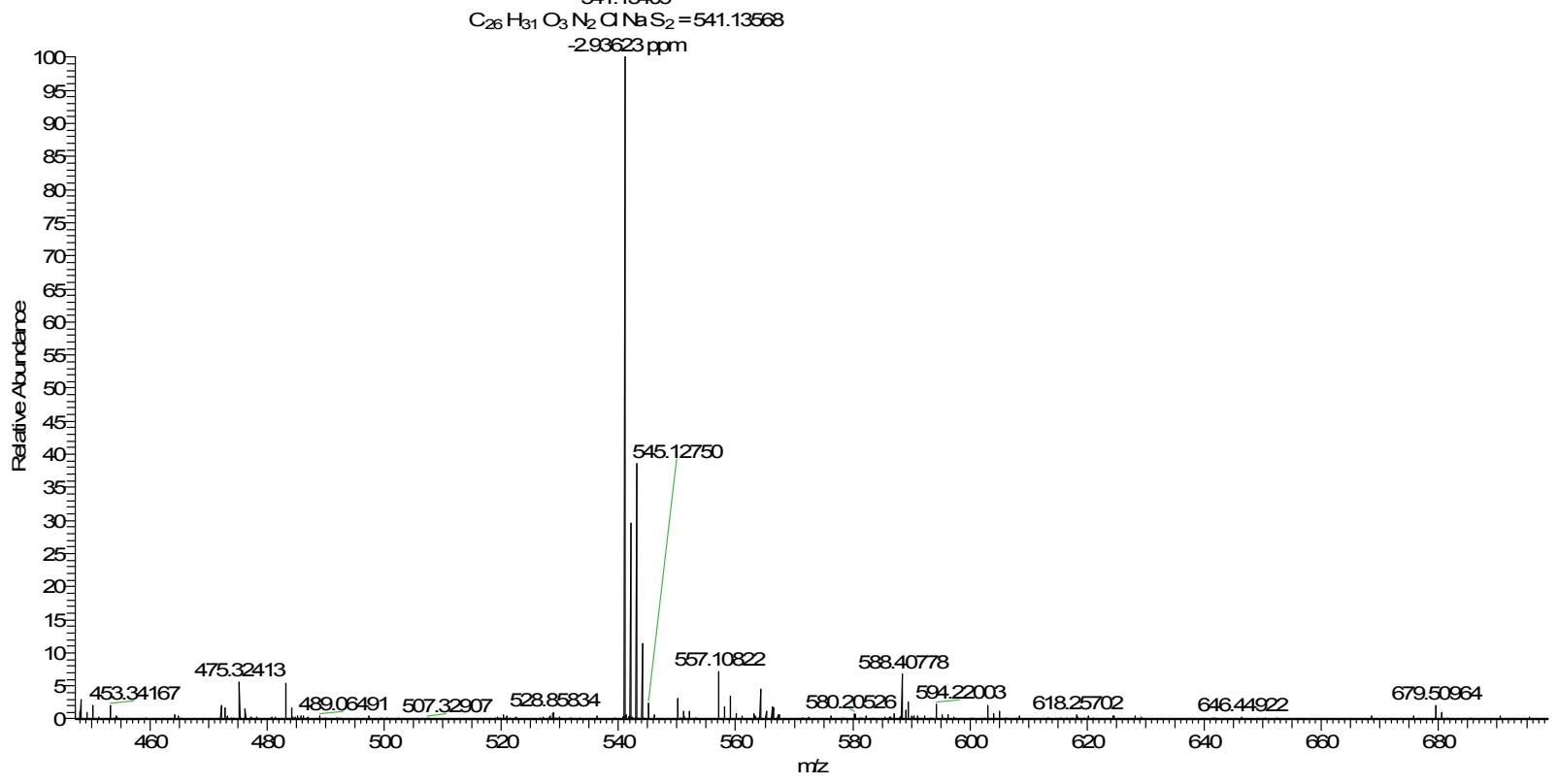

Figure 21. HRMS of compound D3. 


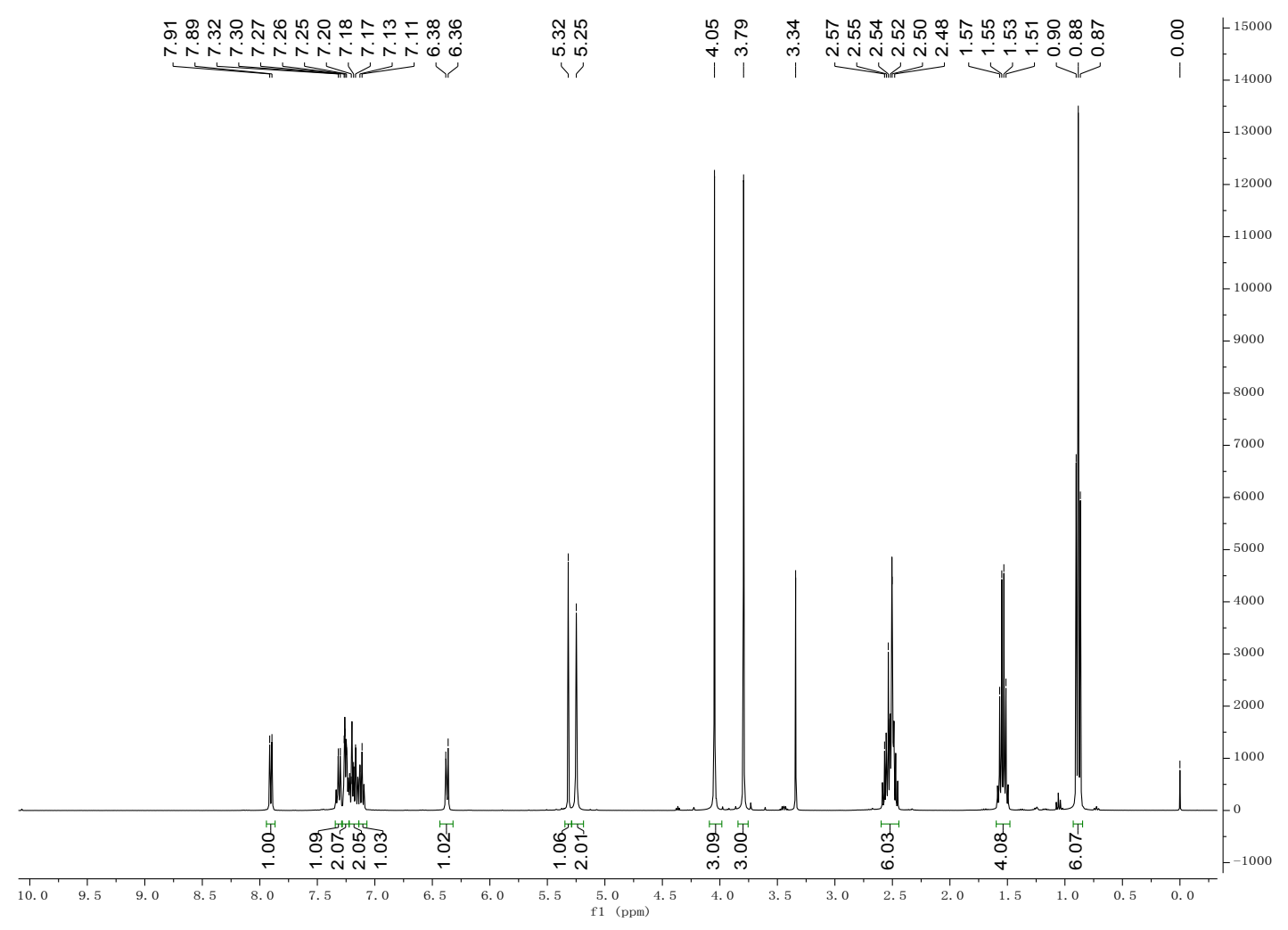

Figure 22. ${ }^{1} \mathrm{H}$ NMR ((400 MHz, DMSO- $\left.d_{6}\right)$ spectrum of compound D4.

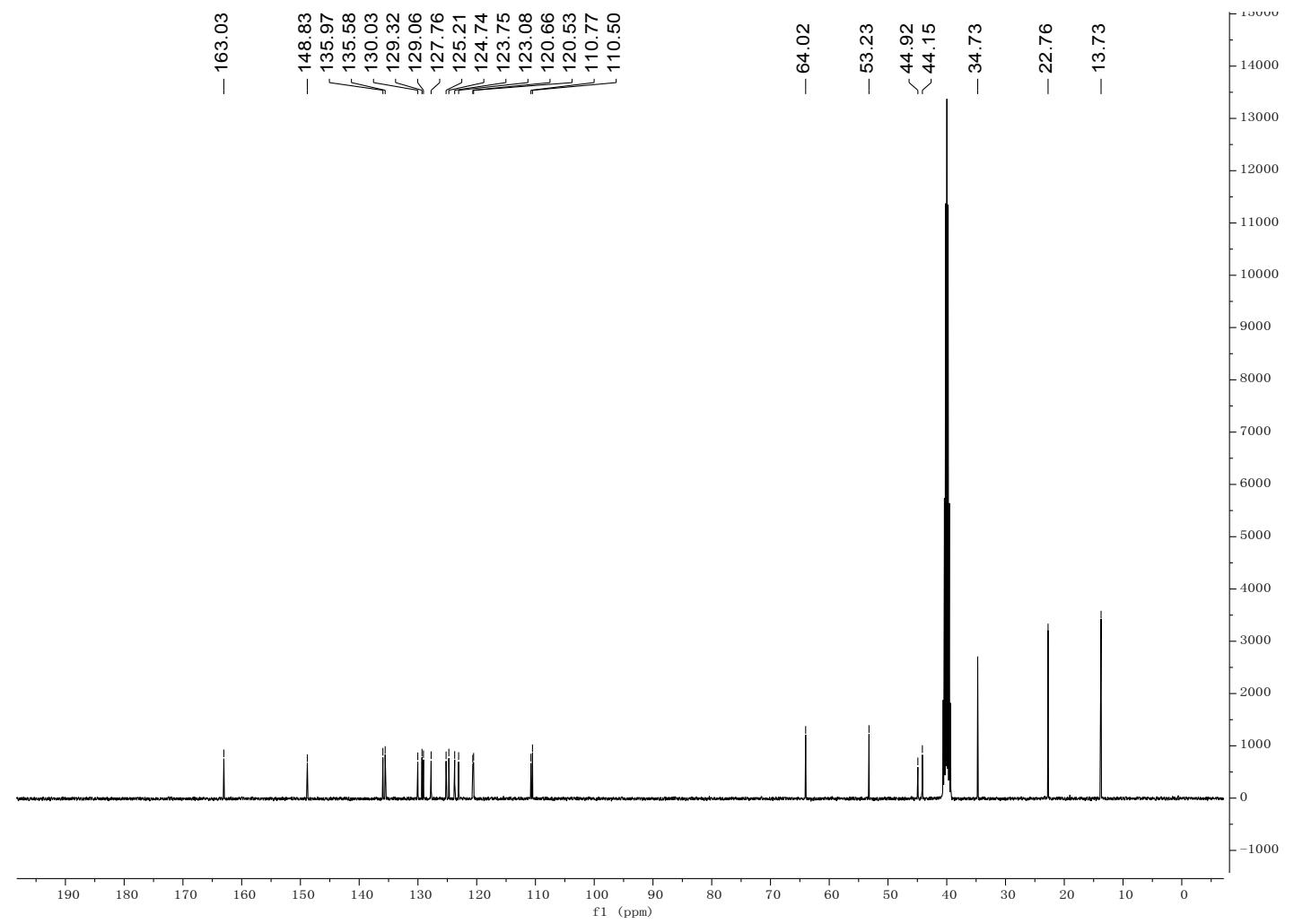

Figure 23. ${ }^{13} \mathrm{C}$ NMR $\left(100 \mathrm{MHz}, \mathrm{DMSO}-d_{6}\right)$ spectrum of compound D4. 
2019041253 \#81 Rा: 0.78 AV: 1 N: 201E8 T: FIMS + p ESI Full ms [100.0000-1000.0000]

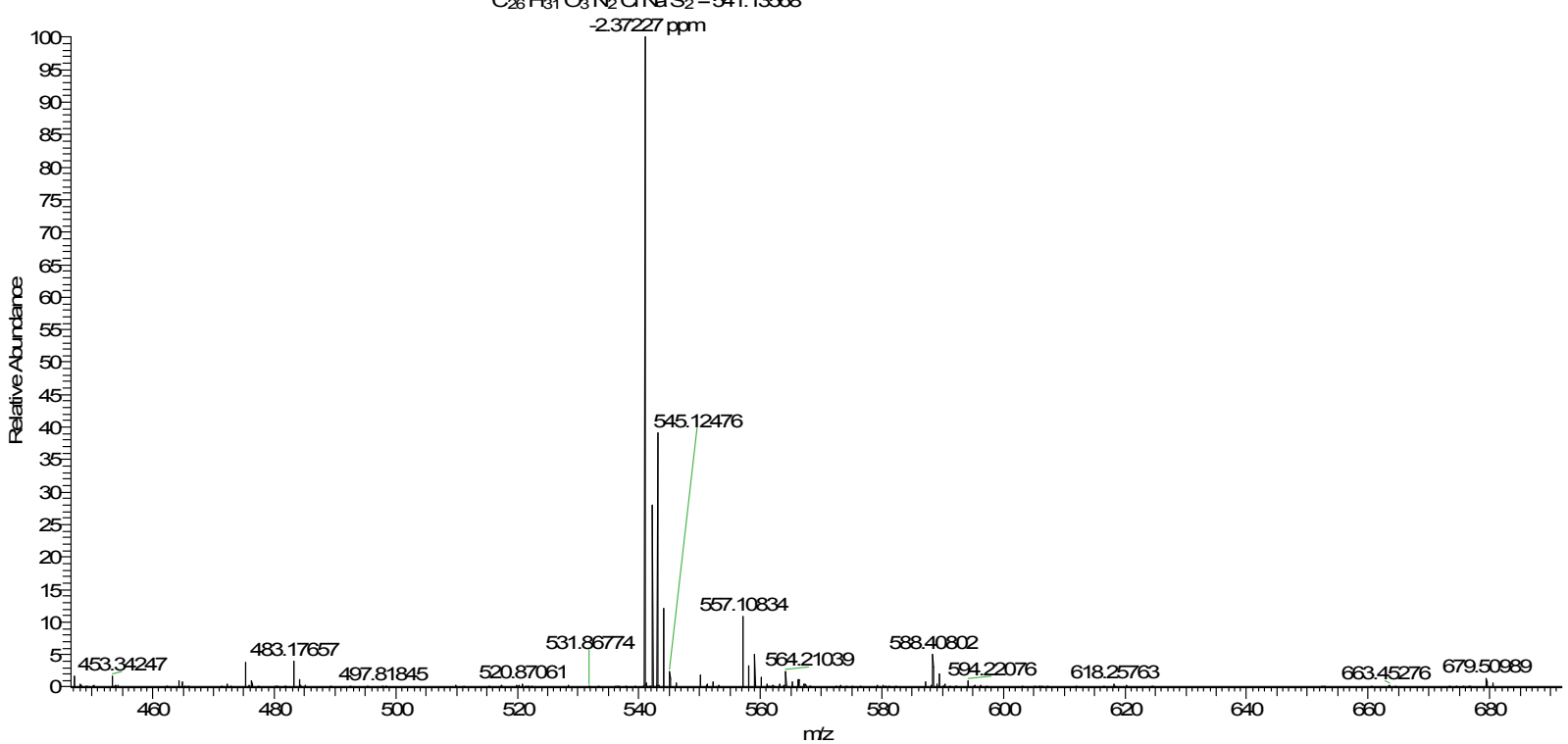

Figure 24. HRMS of compound D4.

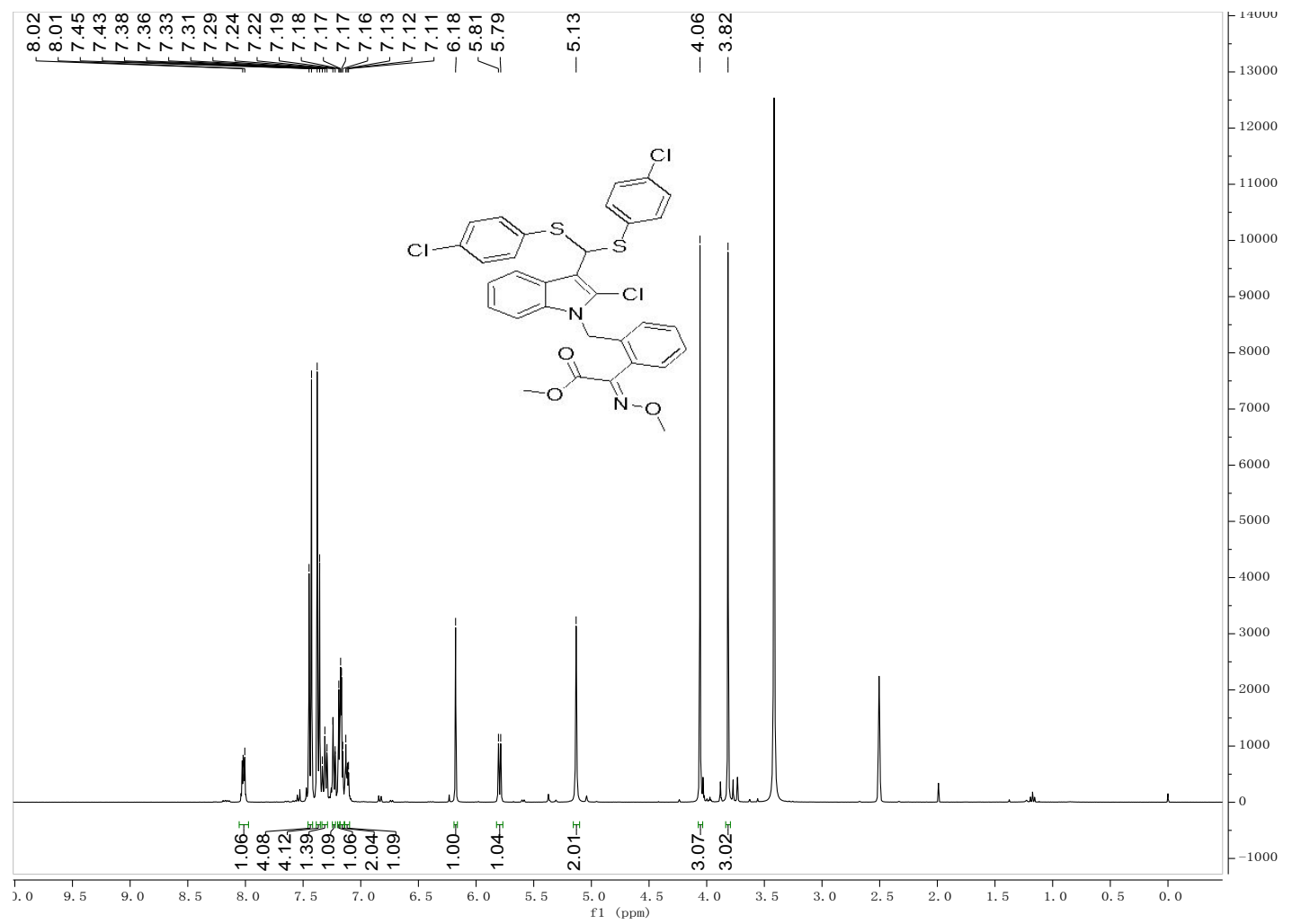

Figure 25. ${ }^{1} \mathrm{H}$ NMR $\left(\left(400 \mathrm{MHz}, \mathrm{DMSO}-d_{6}\right)\right.$ spectrum of compound D5. 


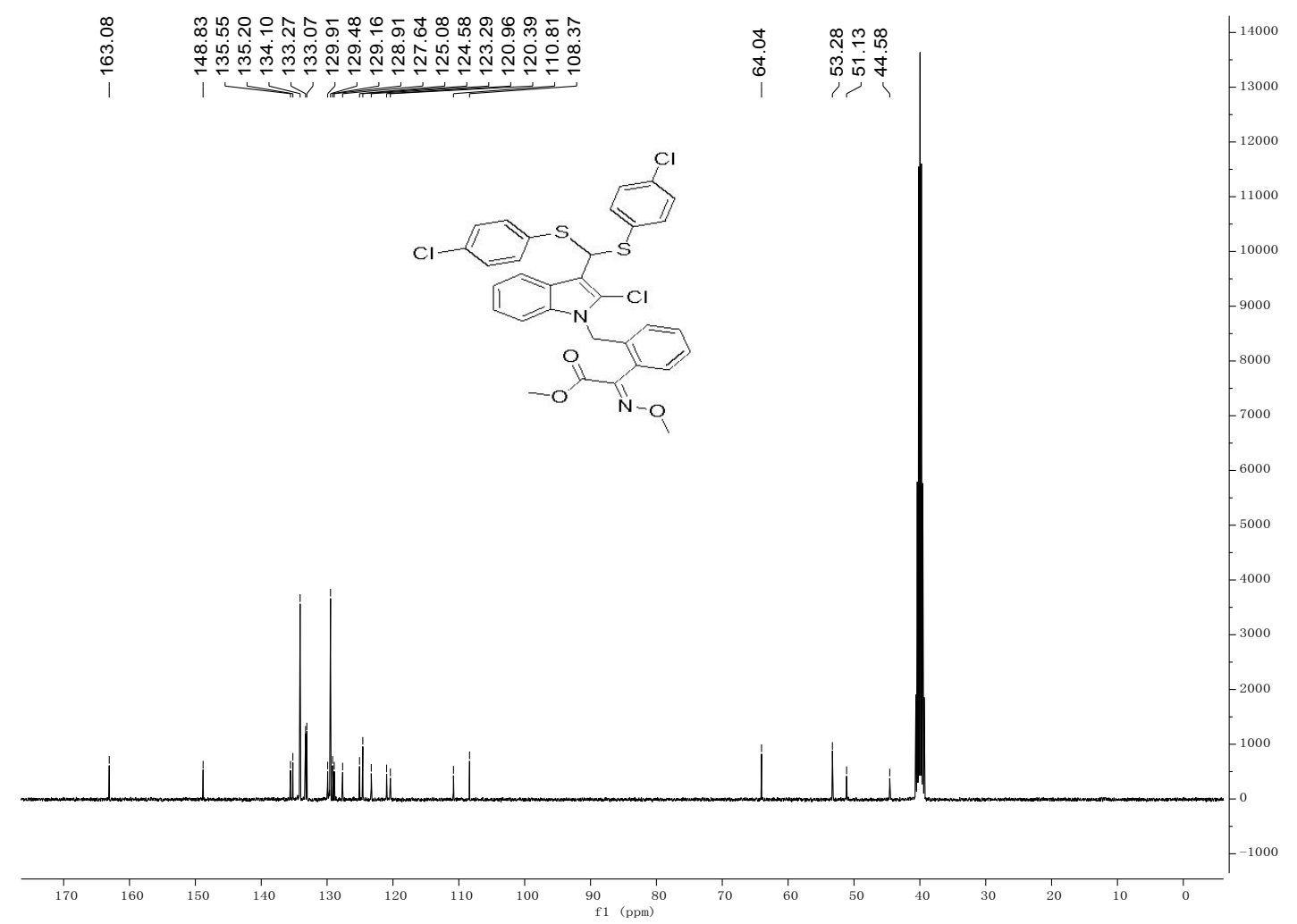

Figure 26. ${ }^{13} \mathrm{C}$ NMR $\left(100 \mathrm{MHz}\right.$, DMSO- $\left.d_{6}\right)$ spectrum of compound D5.

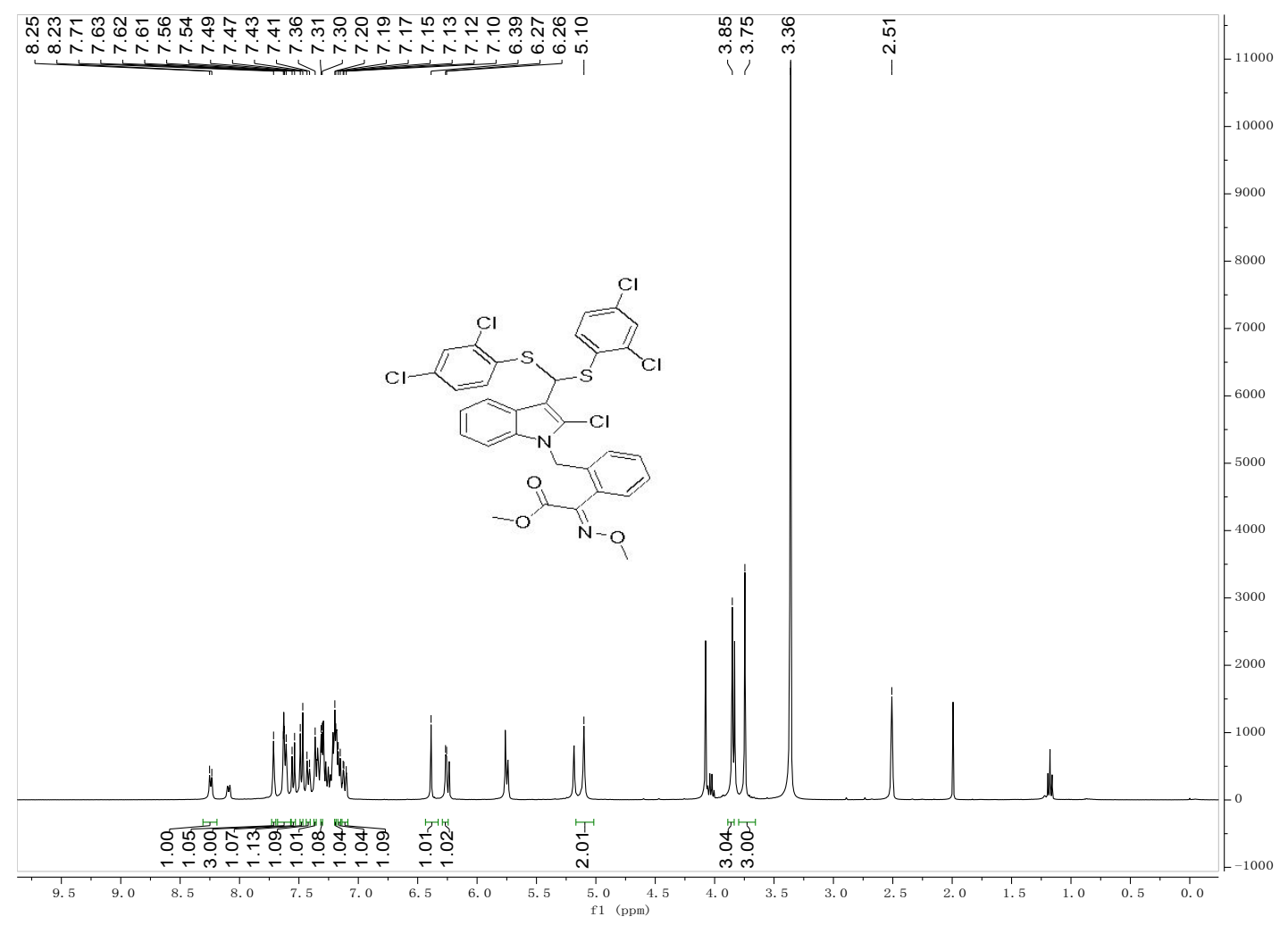

Figure 27. ${ }^{1} \mathrm{H}$ NMR $\left(\left(400 \mathrm{MHz}, \mathrm{DMSO}-d_{6}\right)\right.$ spectrum of compound D6. 


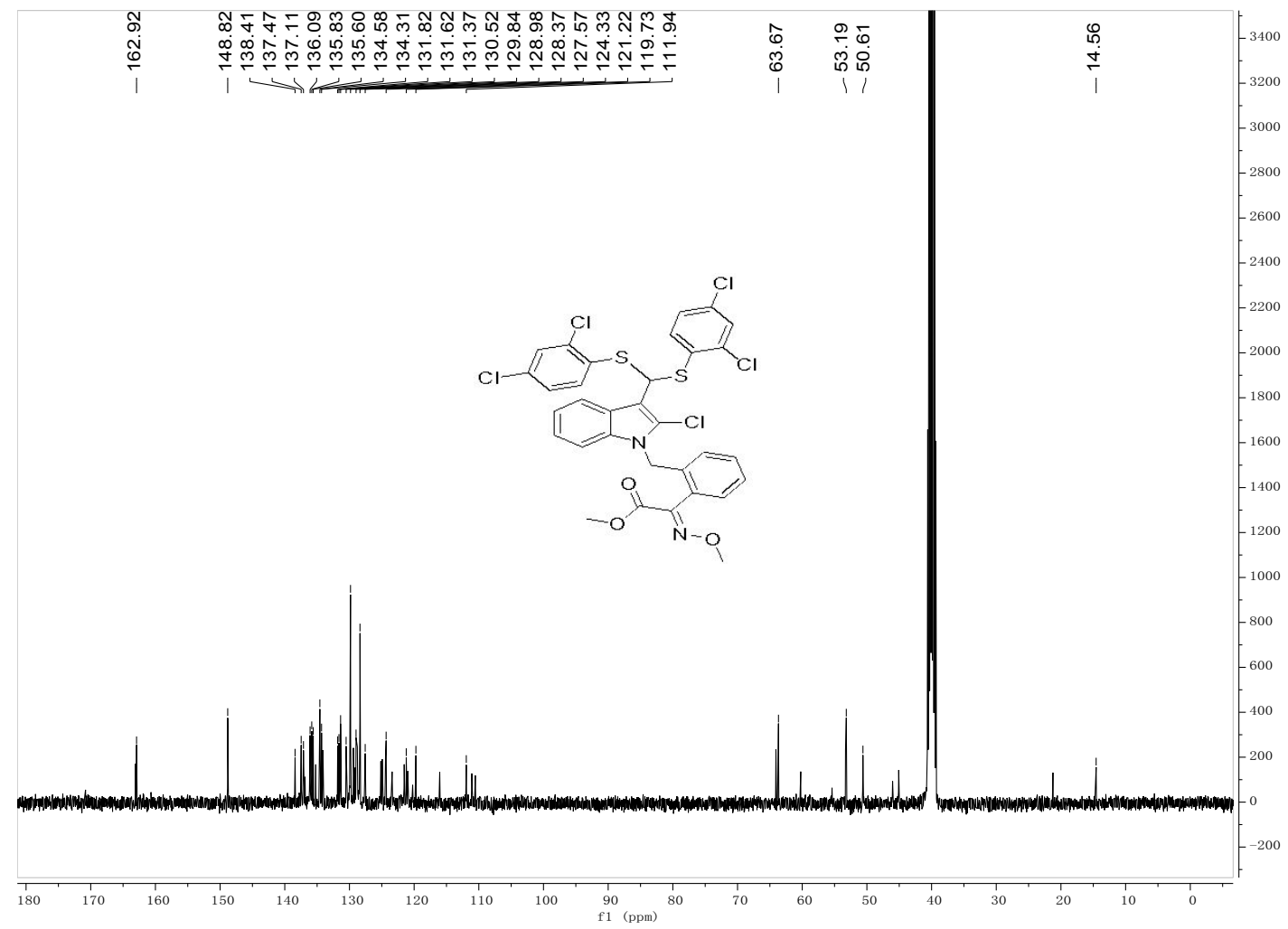

Figure 28. ${ }^{13} \mathrm{C}$ NMR $\left(100 \mathrm{MHz}\right.$, DMSO- $\left.d_{6}\right)$ spectrum of compound D6.

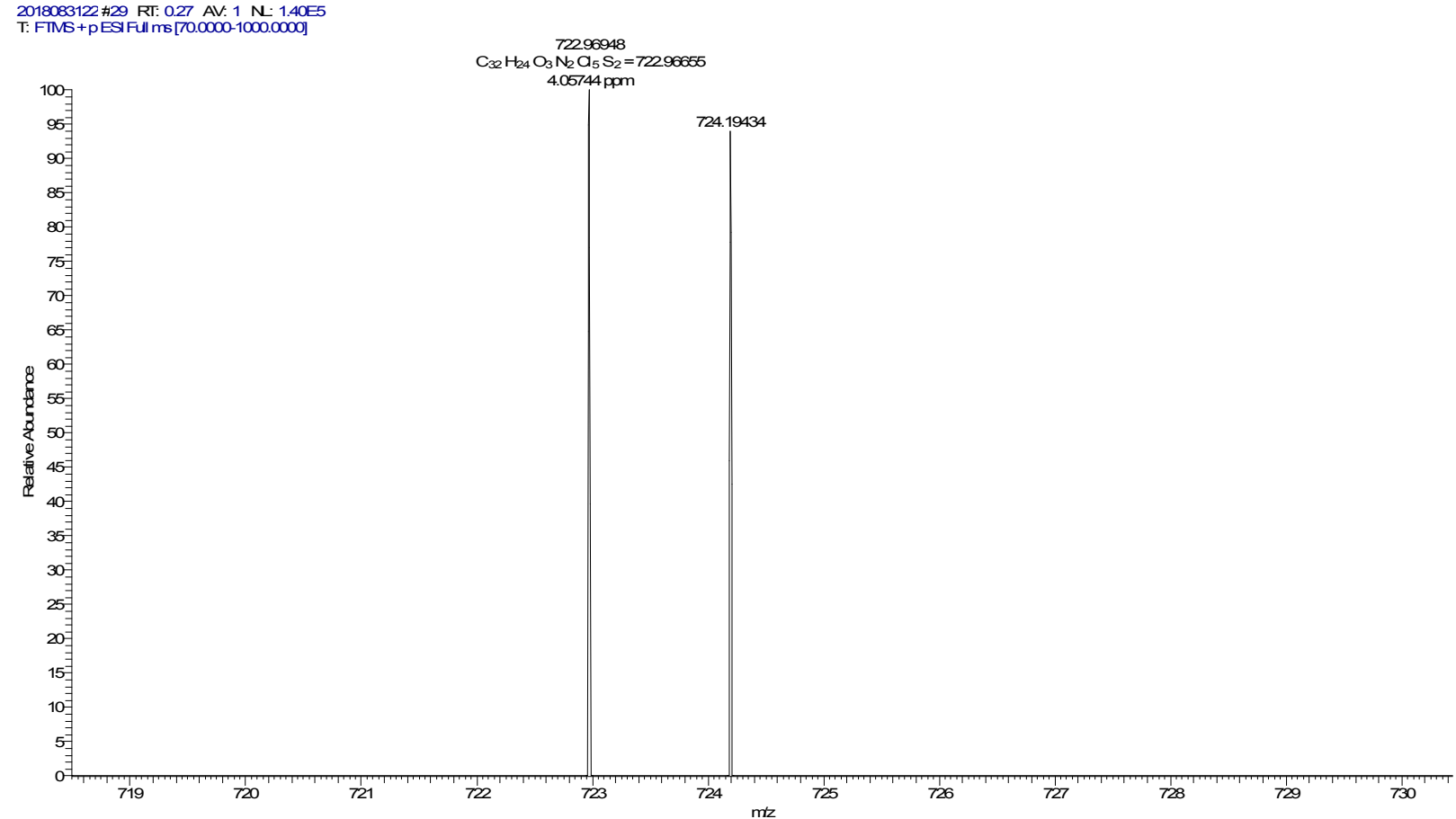

Figure 29. HRMS of compound D6. 


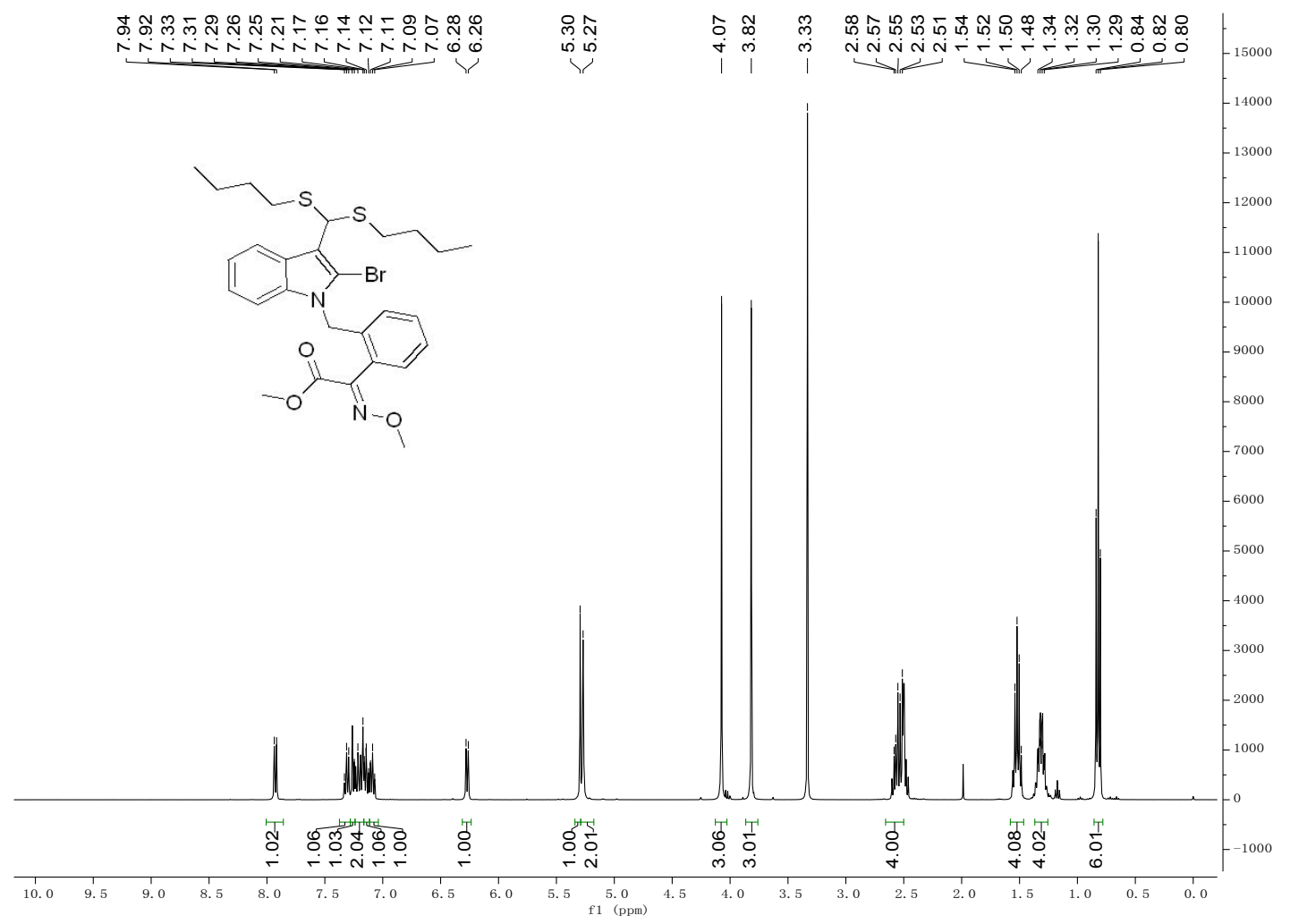

Figure 30. ${ }^{1} \mathrm{H}$ NMR $\left(\left(400 \mathrm{MHz}, \mathrm{DMSO}-d_{6}\right)\right.$ spectrum of compound $\mathbf{D} 7$.

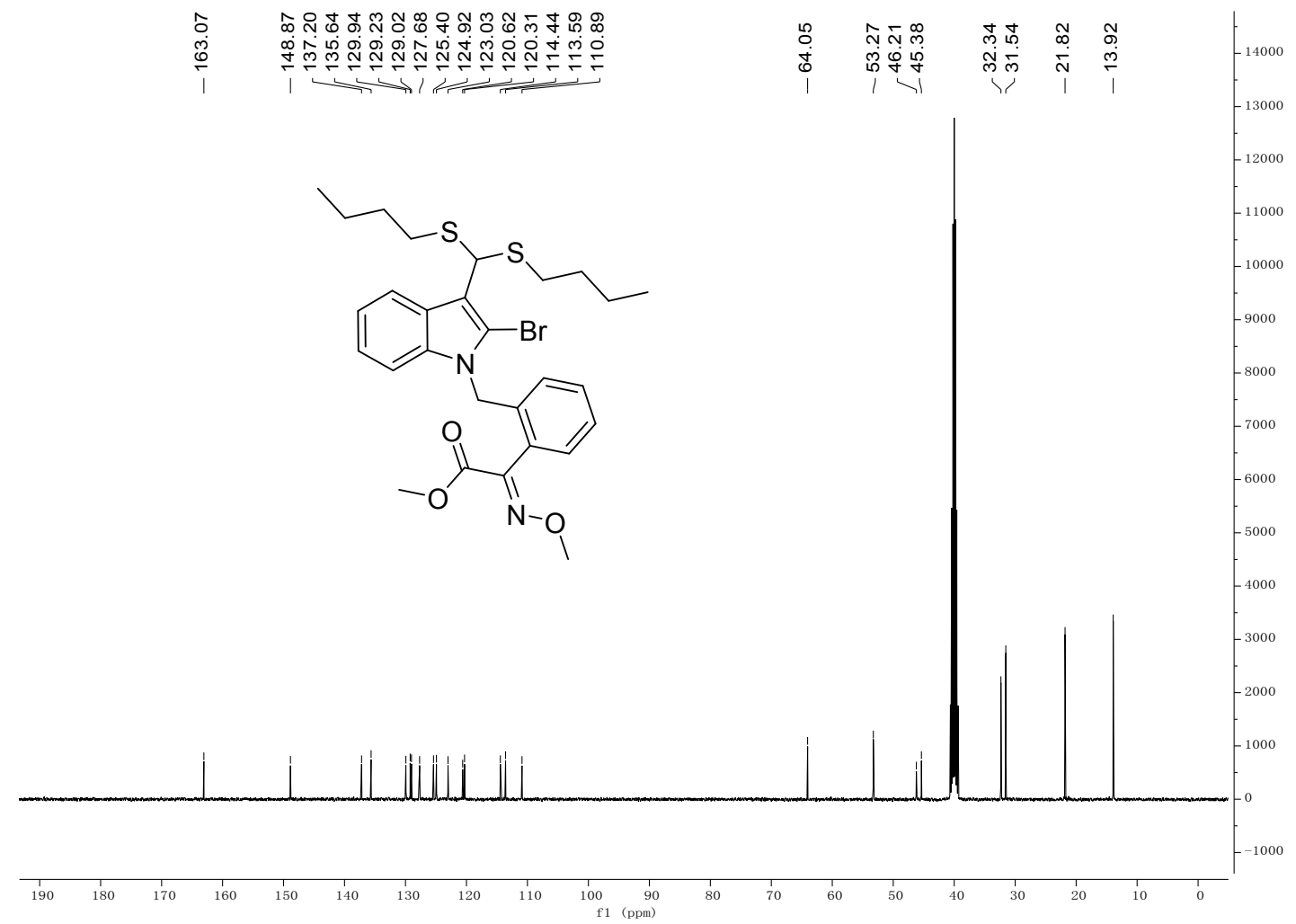

Figure 31. ${ }^{13} \mathrm{C}$ NMR $\left(100 \mathrm{MHz}\right.$, DMSO- $\left.d_{6}\right)$ spectrum of compound D7. 


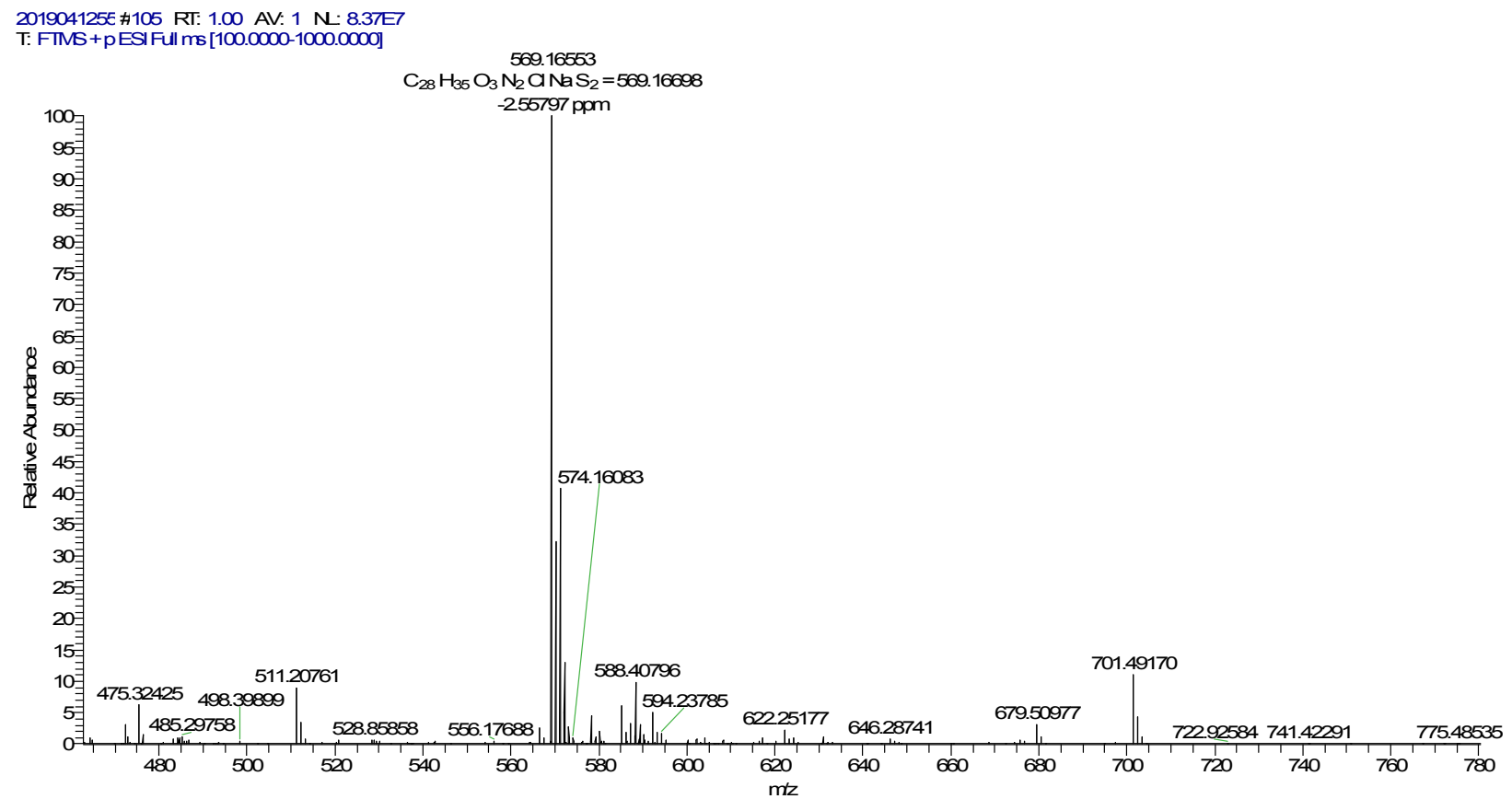

Figure 32. HRMS of compound D7.

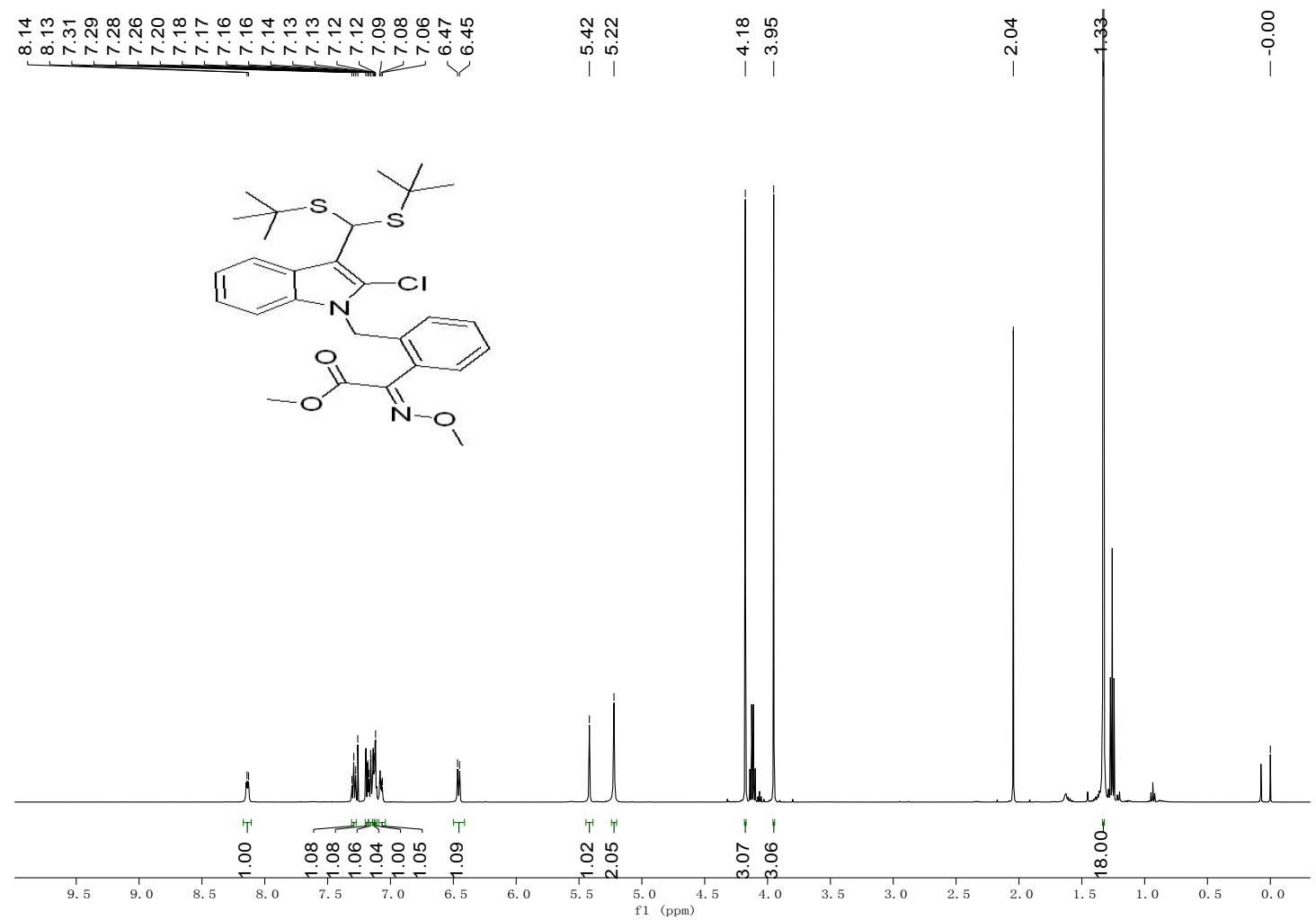

Figure 33. ${ }^{1} \mathrm{H}$ NMR ((400 MHz, DMSO- $\left.d_{6}\right)$ spectrum of compound D8. 

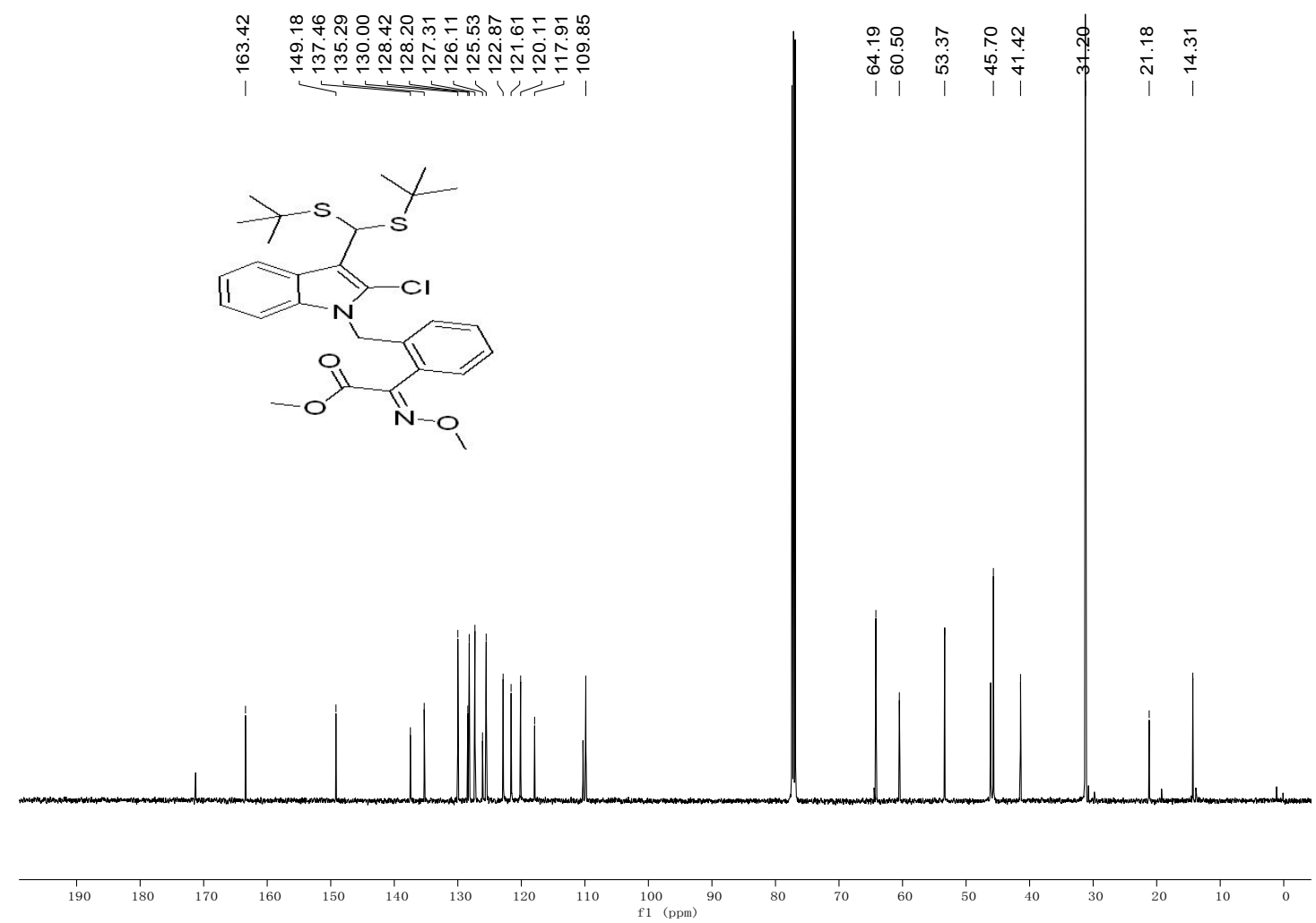

Figure 34. ${ }^{13} \mathrm{C}$ NMR $\left(100 \mathrm{MHz}\right.$, DMSO- $\left.d_{6}\right)$ spectrum of compound D8.

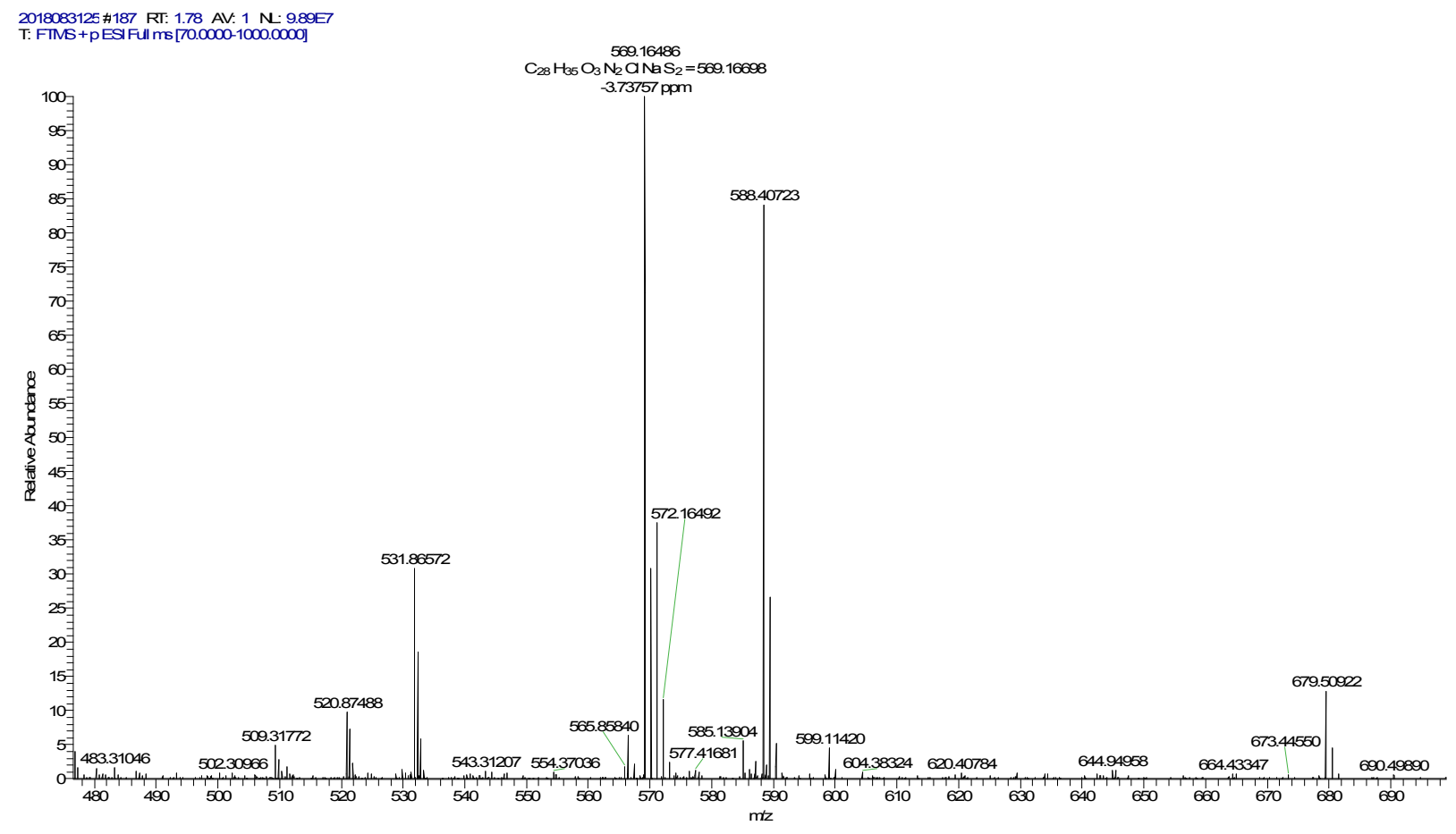

Figure 35. HRMS of compound D8. 


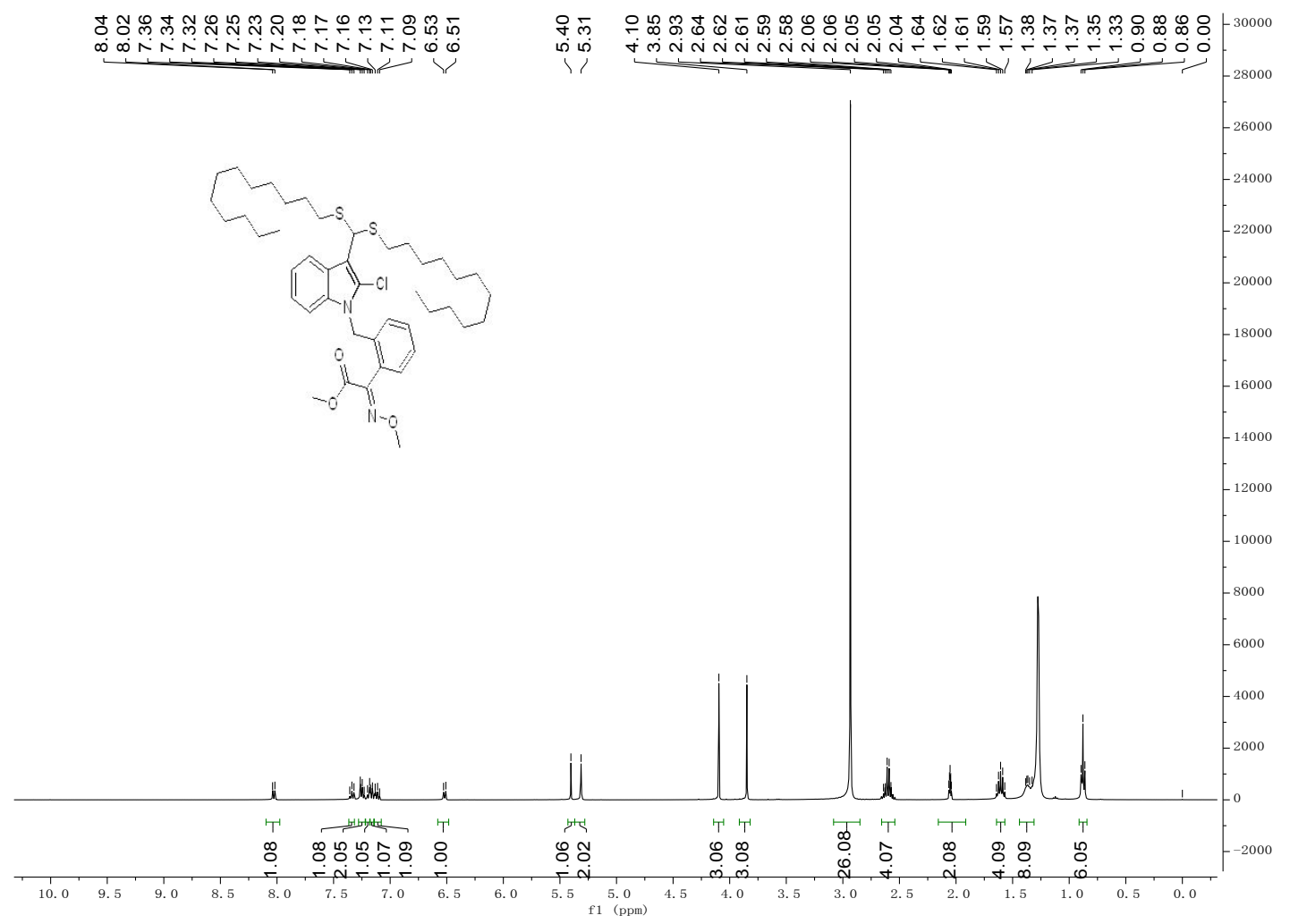

Figure 36. ${ }^{1} \mathrm{H}$ NMR ((400 MHz, DMSO- $\left.d_{6}\right)$ spectrum of compound D9.

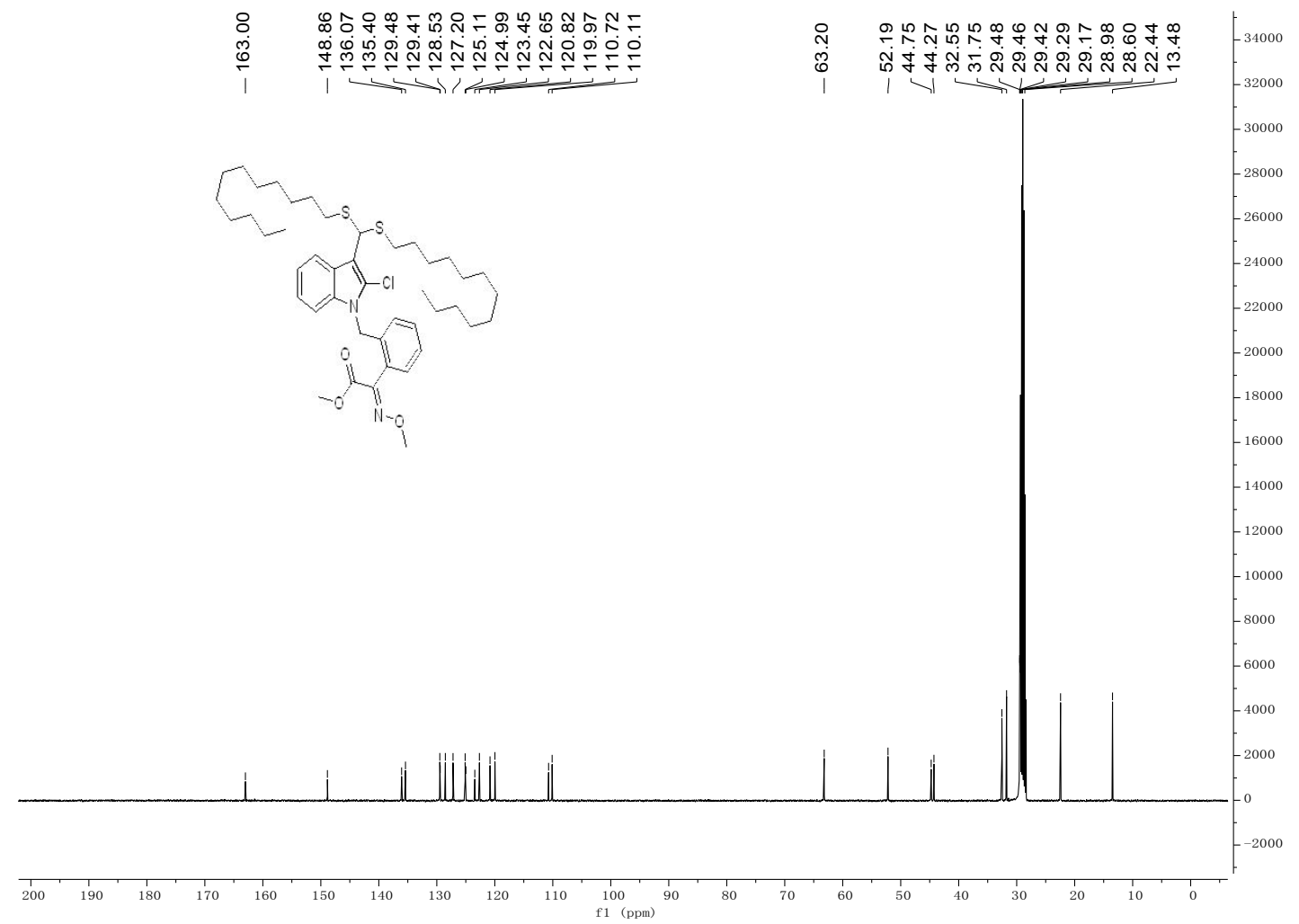

Figure 37. ${ }^{13} \mathrm{C}$ NMR $\left(100 \mathrm{MHz}, \mathrm{DMSO}-d_{6}\right)$ spectrum of compound D9. 


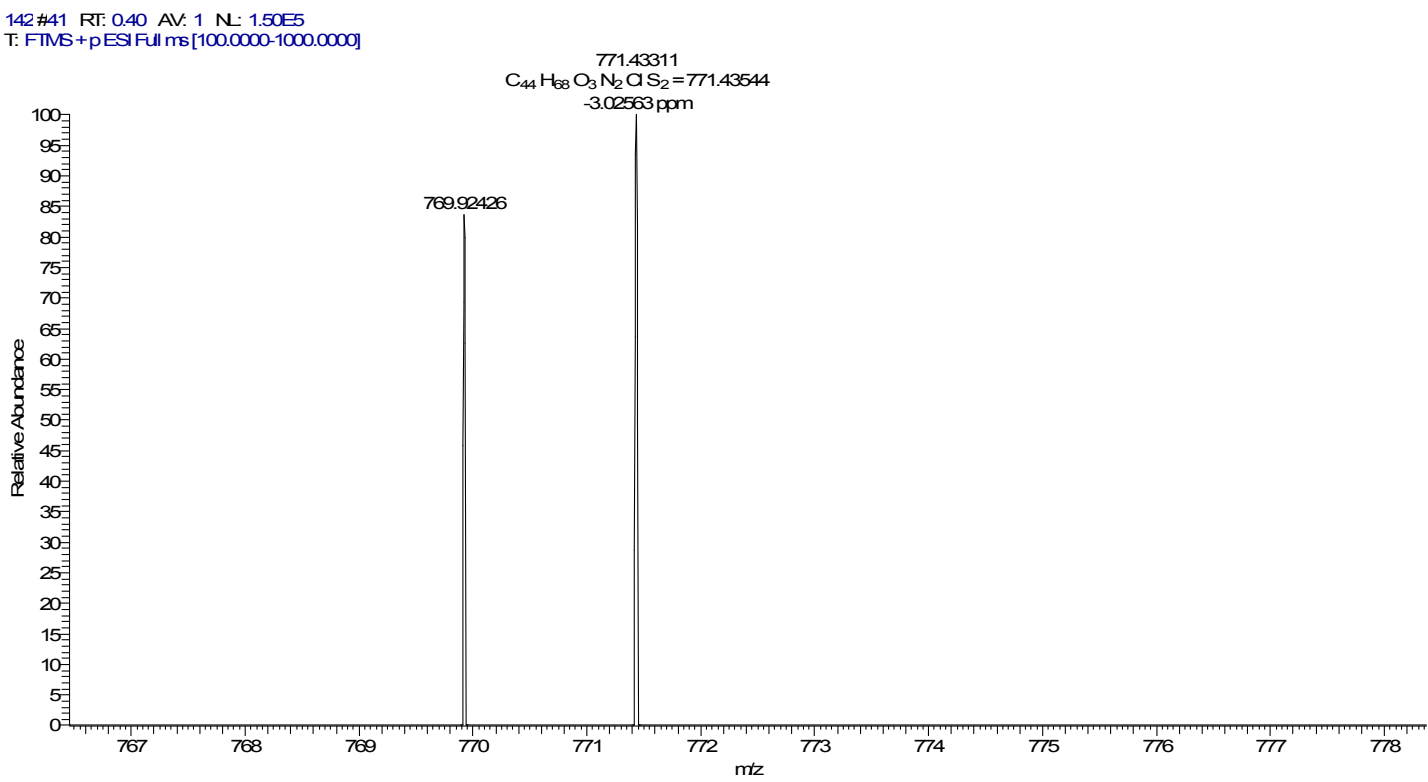

Figure 38. HRMS of compound D9.

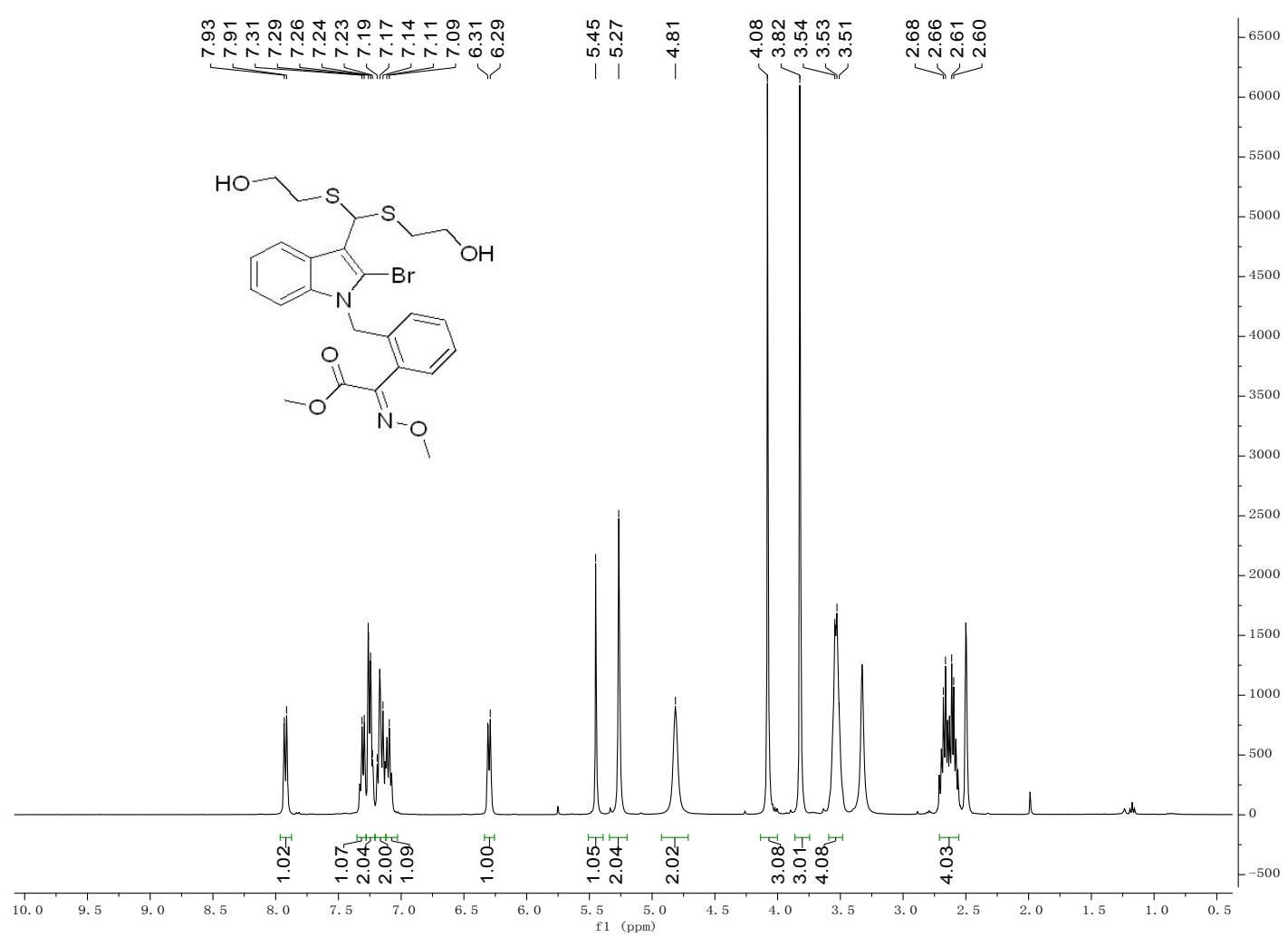

Figure 39. ${ }^{1} \mathrm{H}$ NMR $\left(\left(400 \mathrm{MHz}, \mathrm{DMSO}-d_{6}\right)\right.$ spectrum of compound D10. 


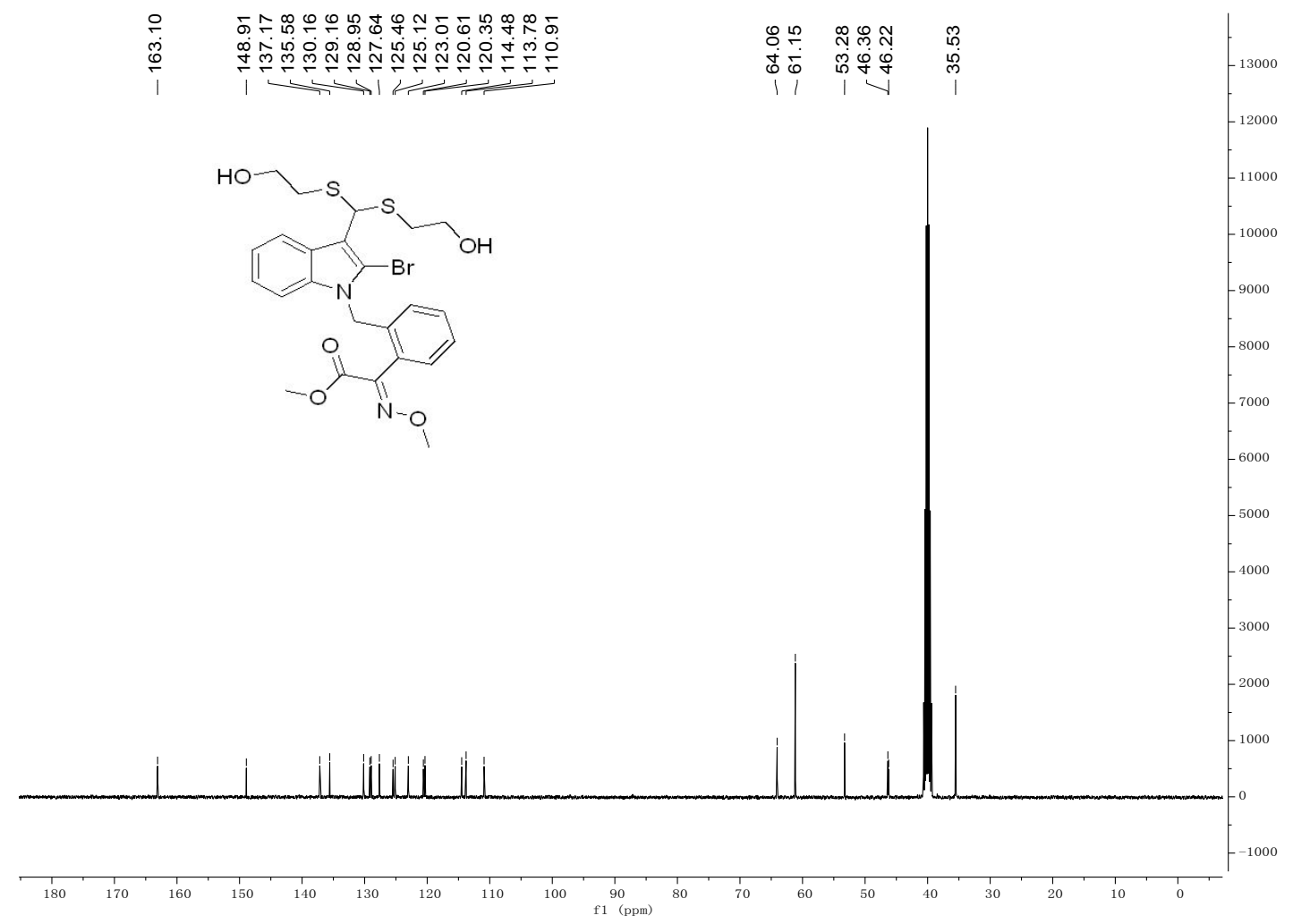

Figure 40. ${ }^{13} \mathrm{C}$ NMR (100 MHz, DMSO- $\left.d_{6}\right)$ spectrum of compound D10.

2018093003 \#183 RI: 0.80 AV: 1 N.-1.72E]
T: FTMS + pESIFul ms [100.0000-1000.0000]

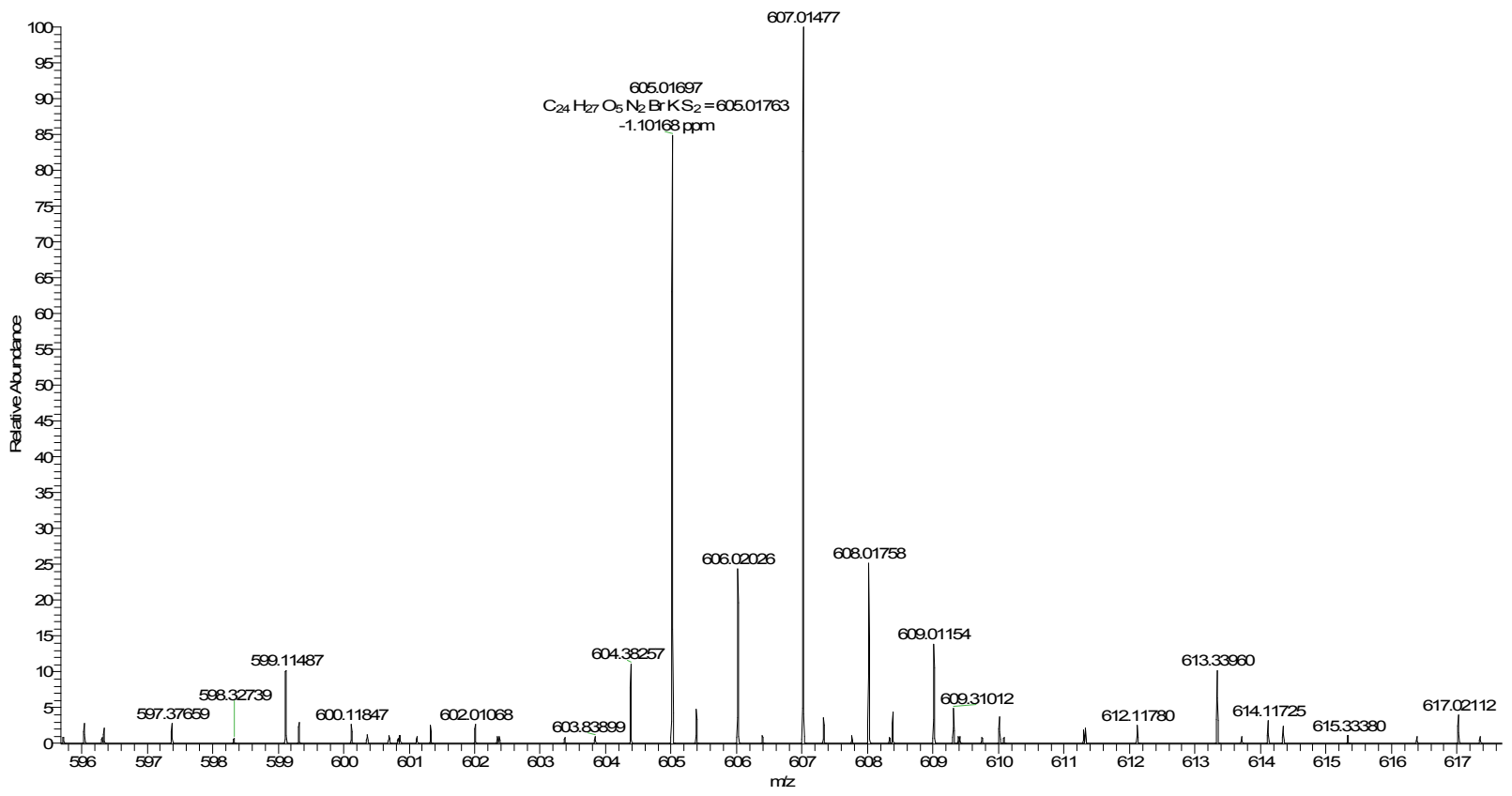

Figure 41. HRMS of compound D10. 


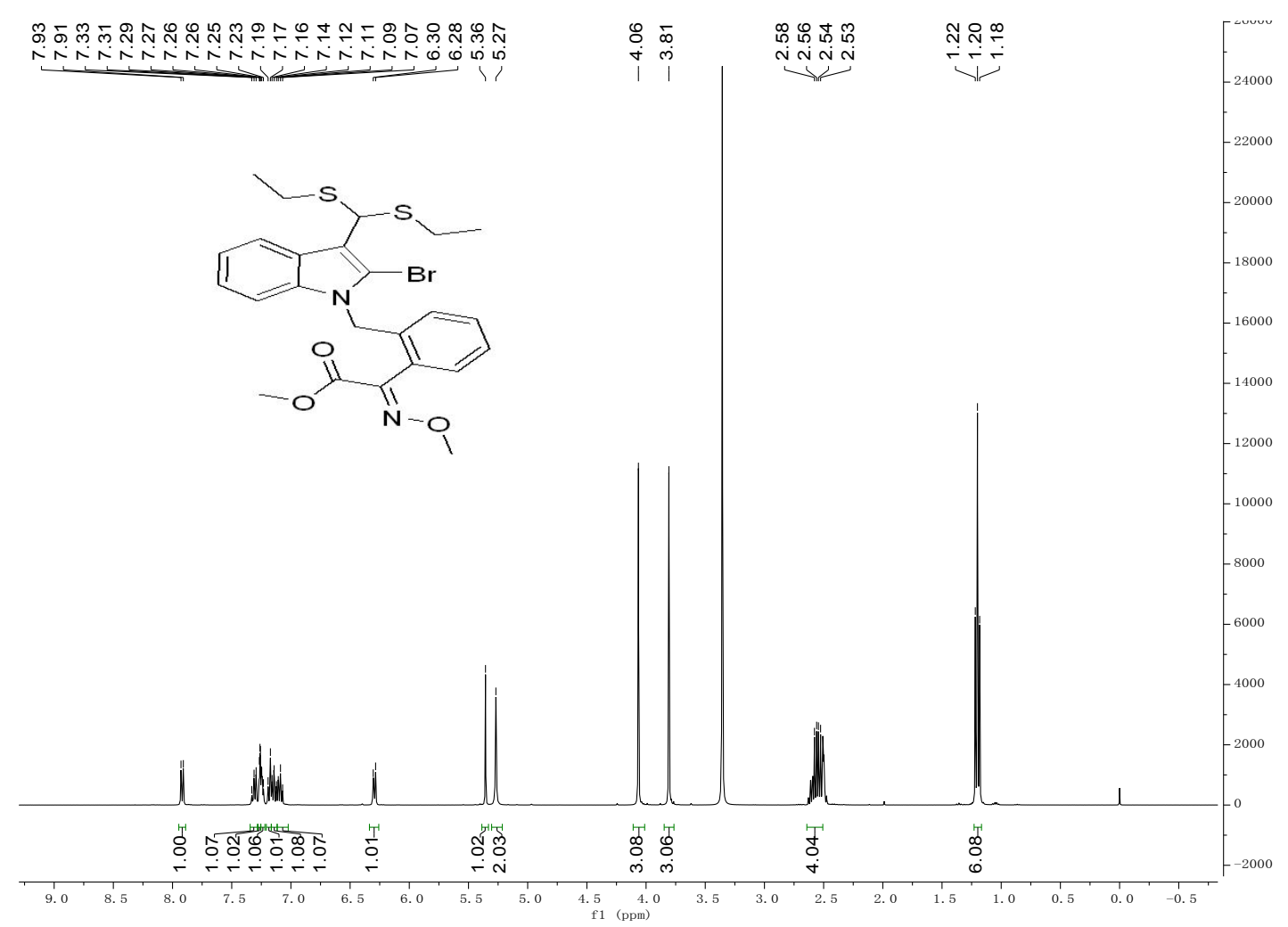

Figure 42. ${ }^{1} \mathrm{H}$ NMR ((400 MHz, DMSO- $\left.d_{6}\right)$ spectrum of compound D11.

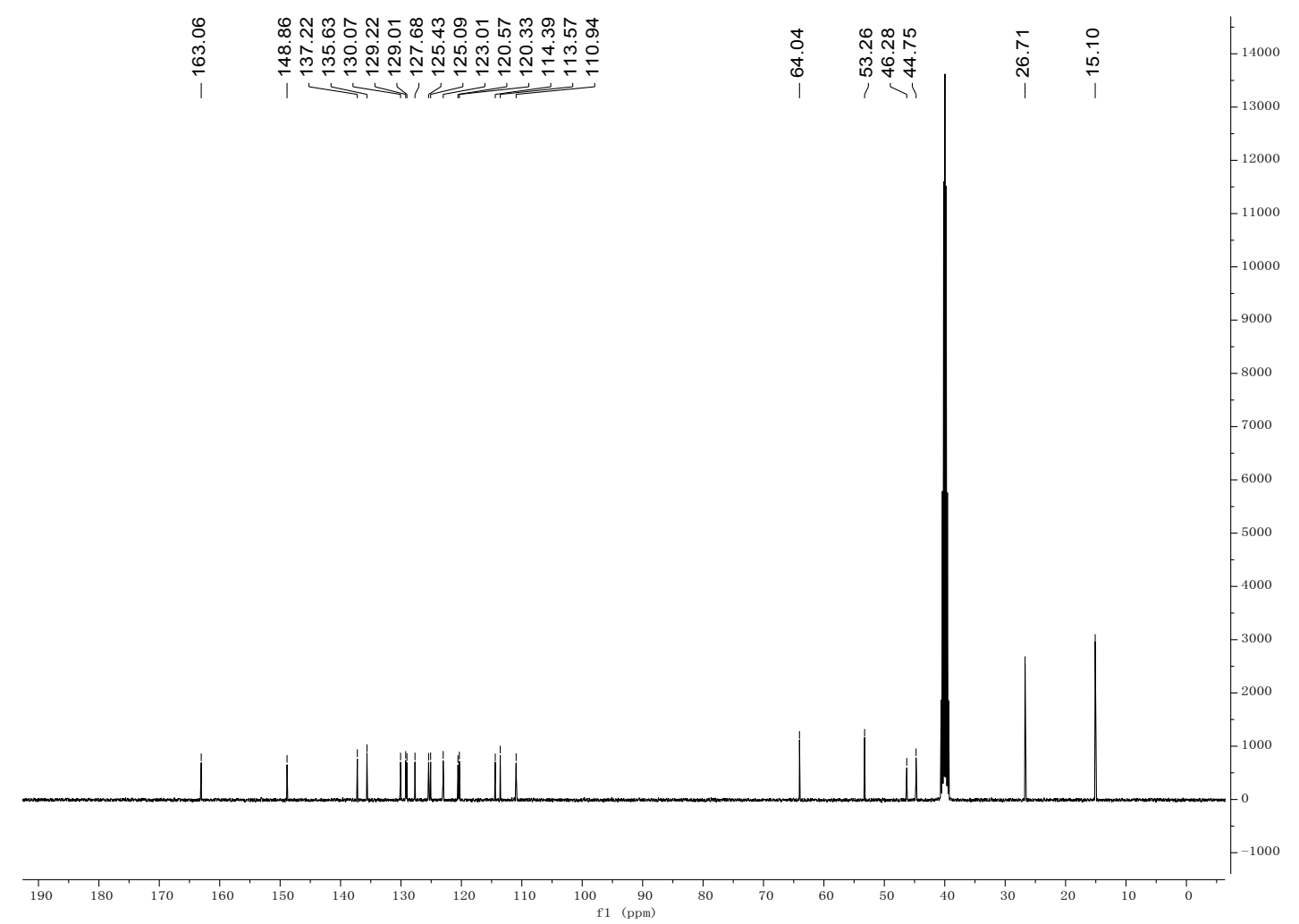

Figure 43. ${ }^{13} \mathrm{C}$ NMR (100 MHz, DMSO- $\left.d_{6}\right)$ spectrum of compound D11. 


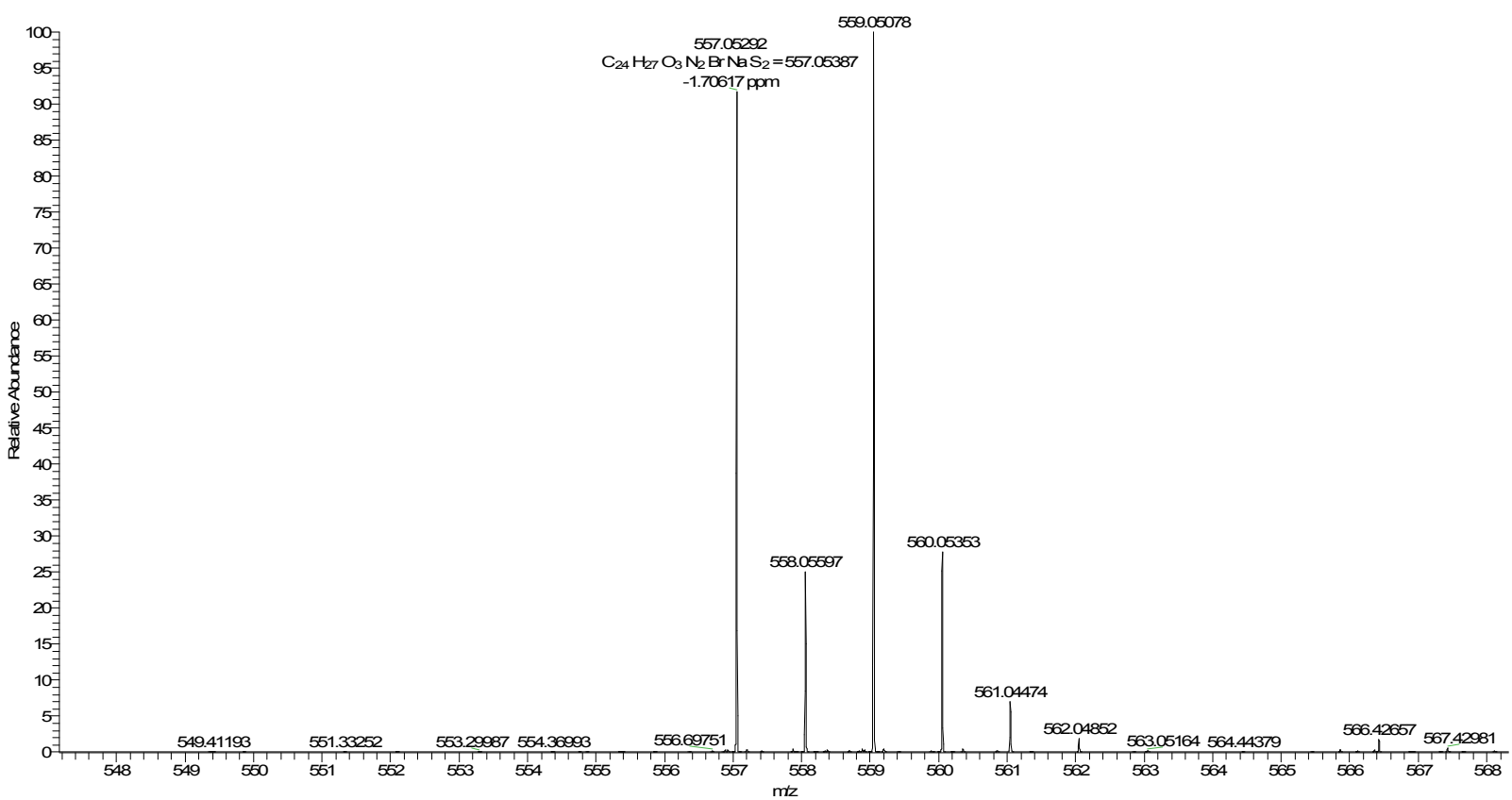

Figure 44. HRMS of compound D11.

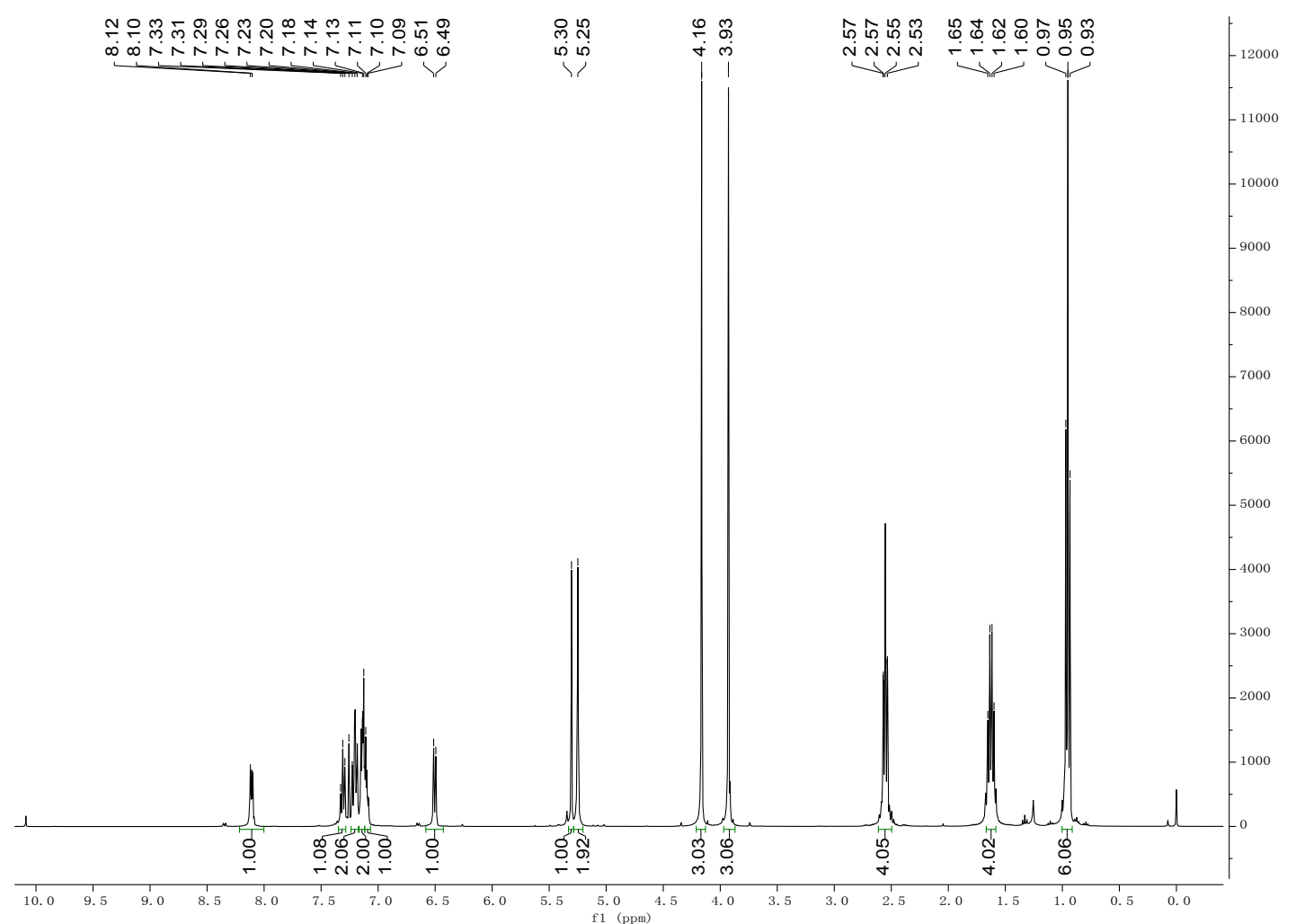

Figure 45. ${ }^{1} \mathrm{H}$ NMR ((400 MHz, DMSO- $\left.d_{6}\right)$ spectrum of compound D12. 


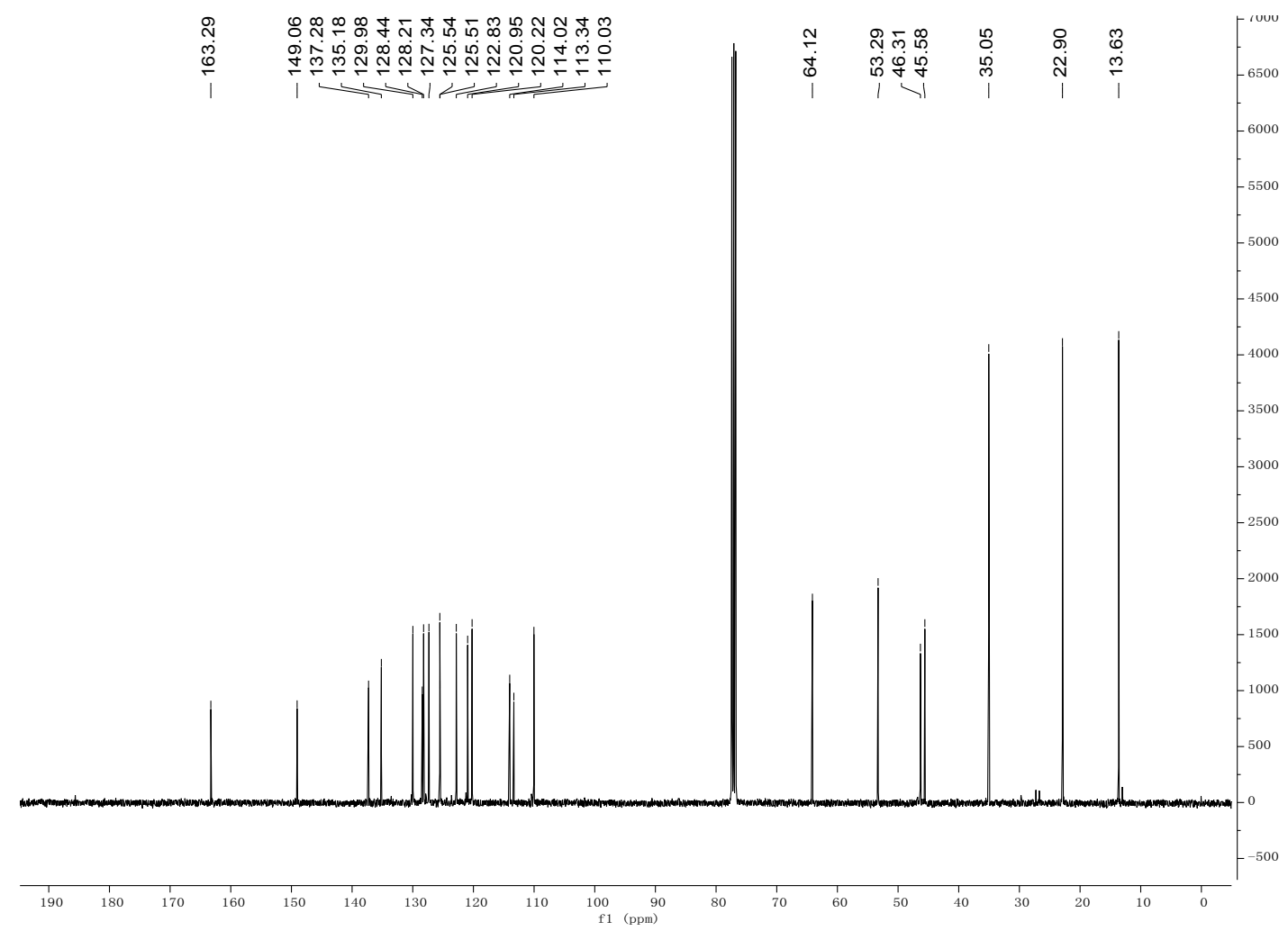

Figure 46. ${ }^{13} \mathrm{C}$ NMR (100 MHz, DMSO- $\left.d_{6}\right)$ spectrum of compound D12.

201904125/ \#83 Rा: 0.79 AV: 1 N: 9.86E T: FIMS + pESI Ful ms [100.0000-1000.0000]

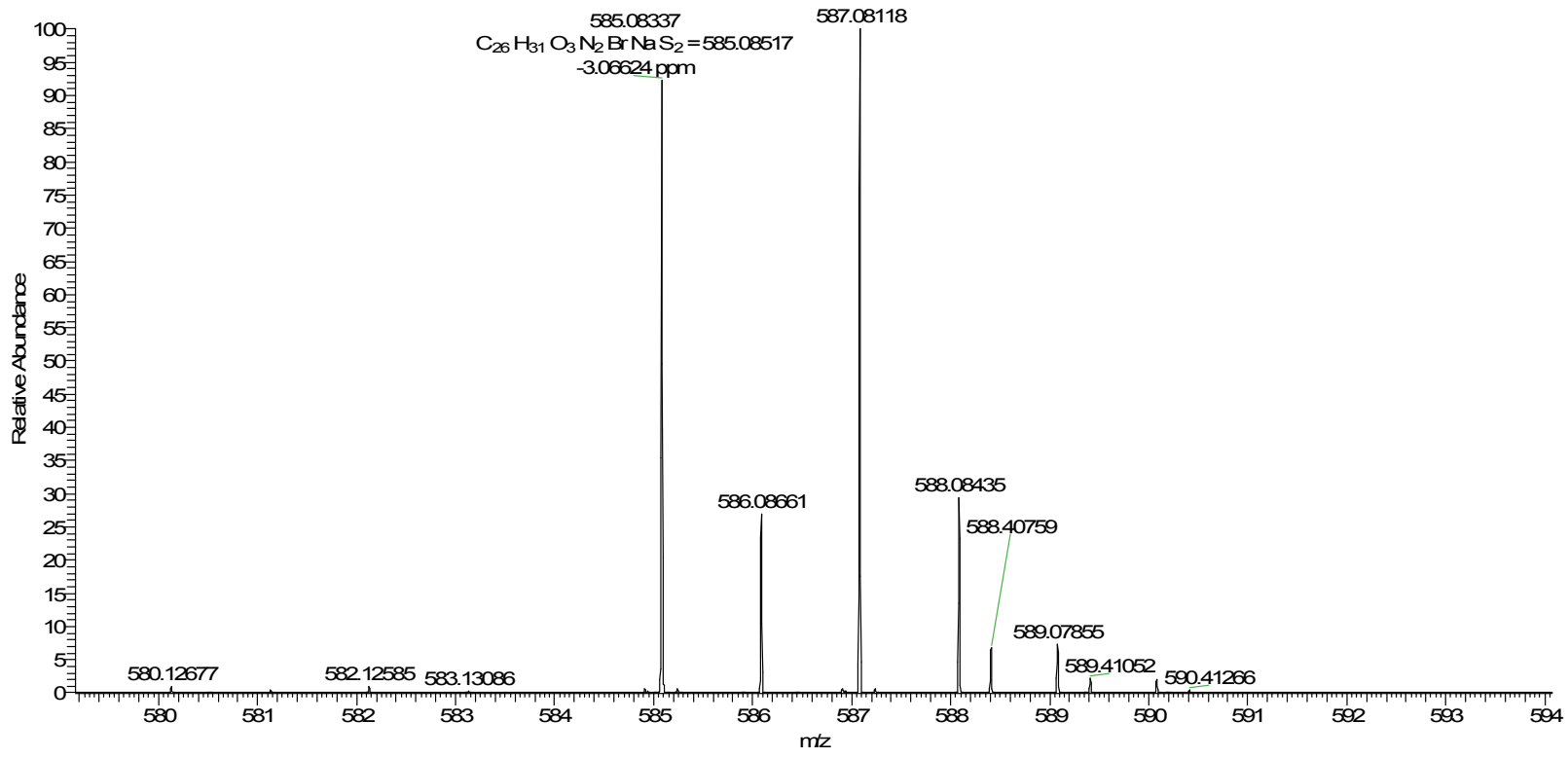

Figure 47. HRMS of compound D12. 


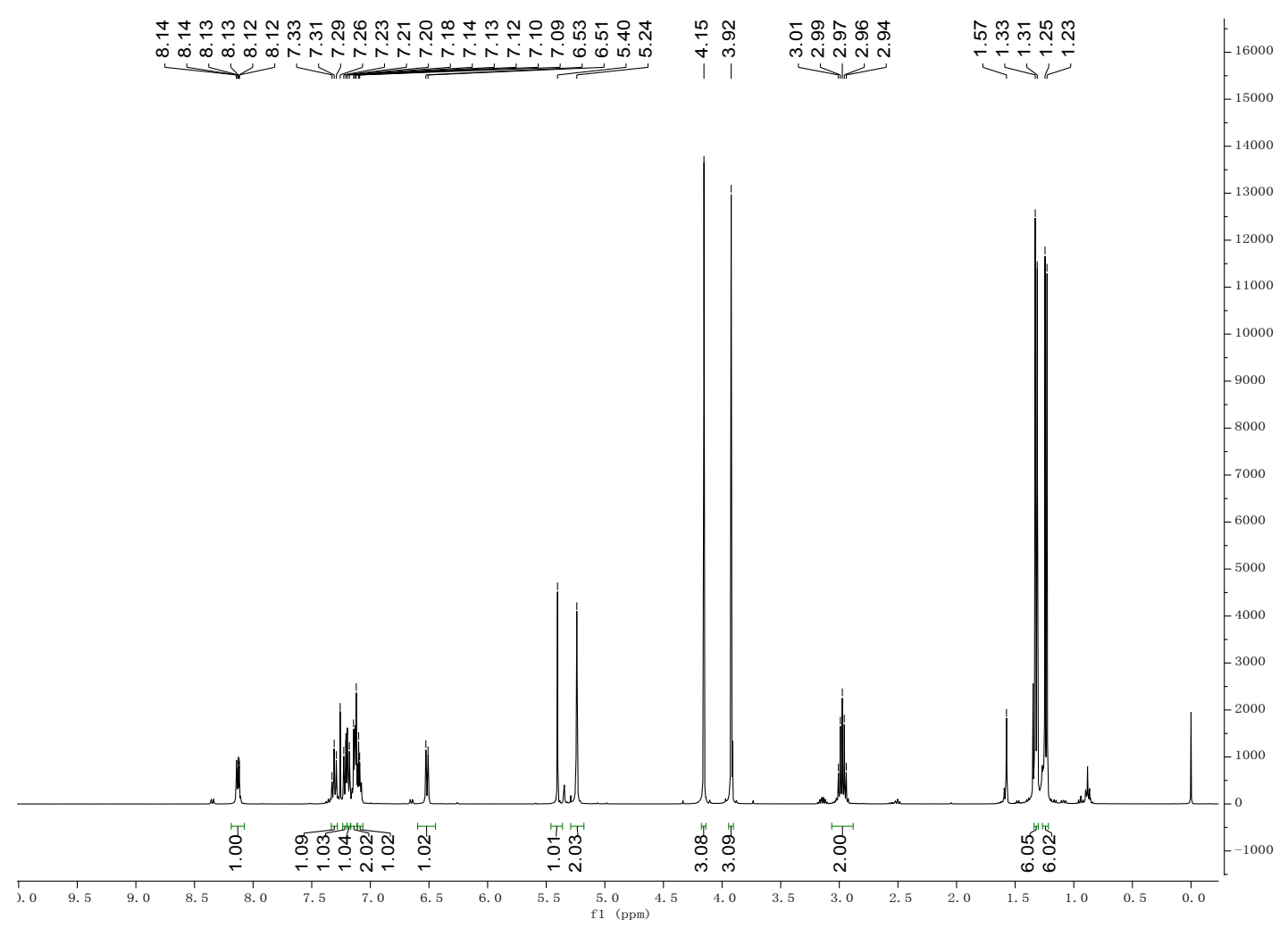

Figure 48. ${ }^{1} \mathrm{H}$ NMR ((400 MHz, DMSO- $\left.d_{6}\right)$ spectrum of compound D13.

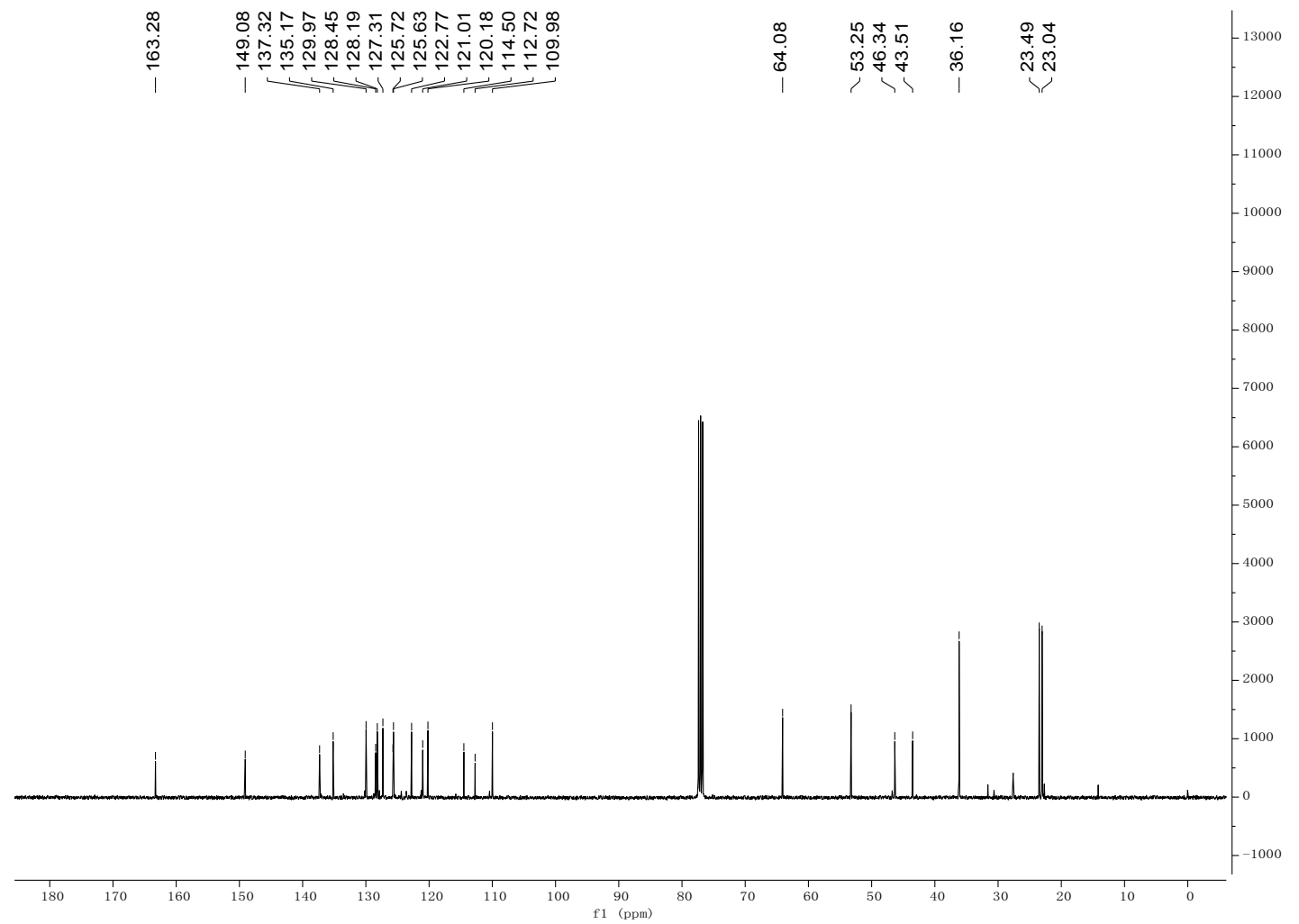

Figure 49. ${ }^{13} \mathrm{C}$ NMR (100 MHz, DMSO- $d_{6}$ ) spectrum of compound D13. 
2019041258 \#77 Rा: 0.74 AV: 1 N: 5.59E7 T: FIMS + pESI Full ms [100.0000-1000.0000]

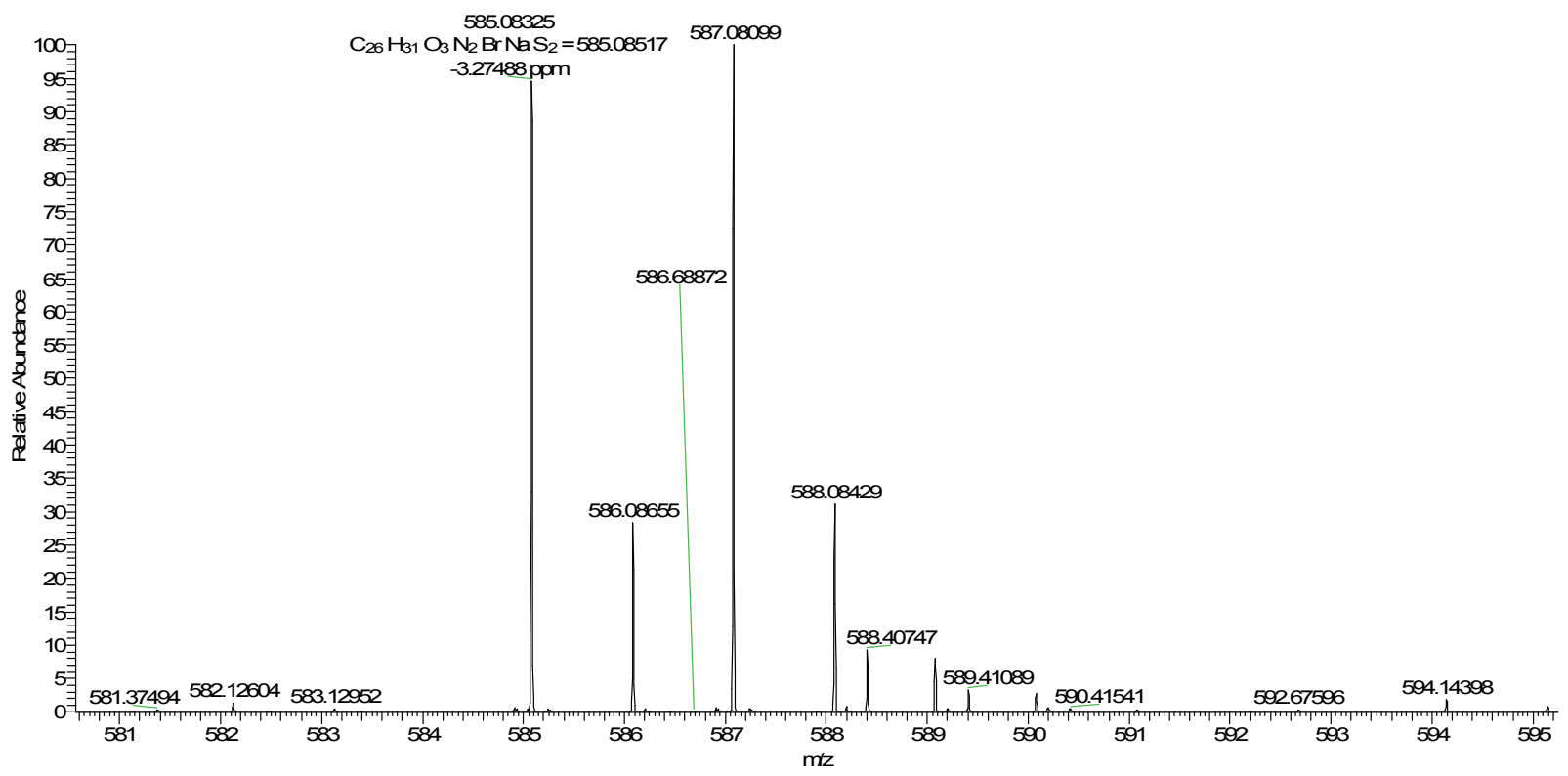

Figure 50. HRMS of compound D13.

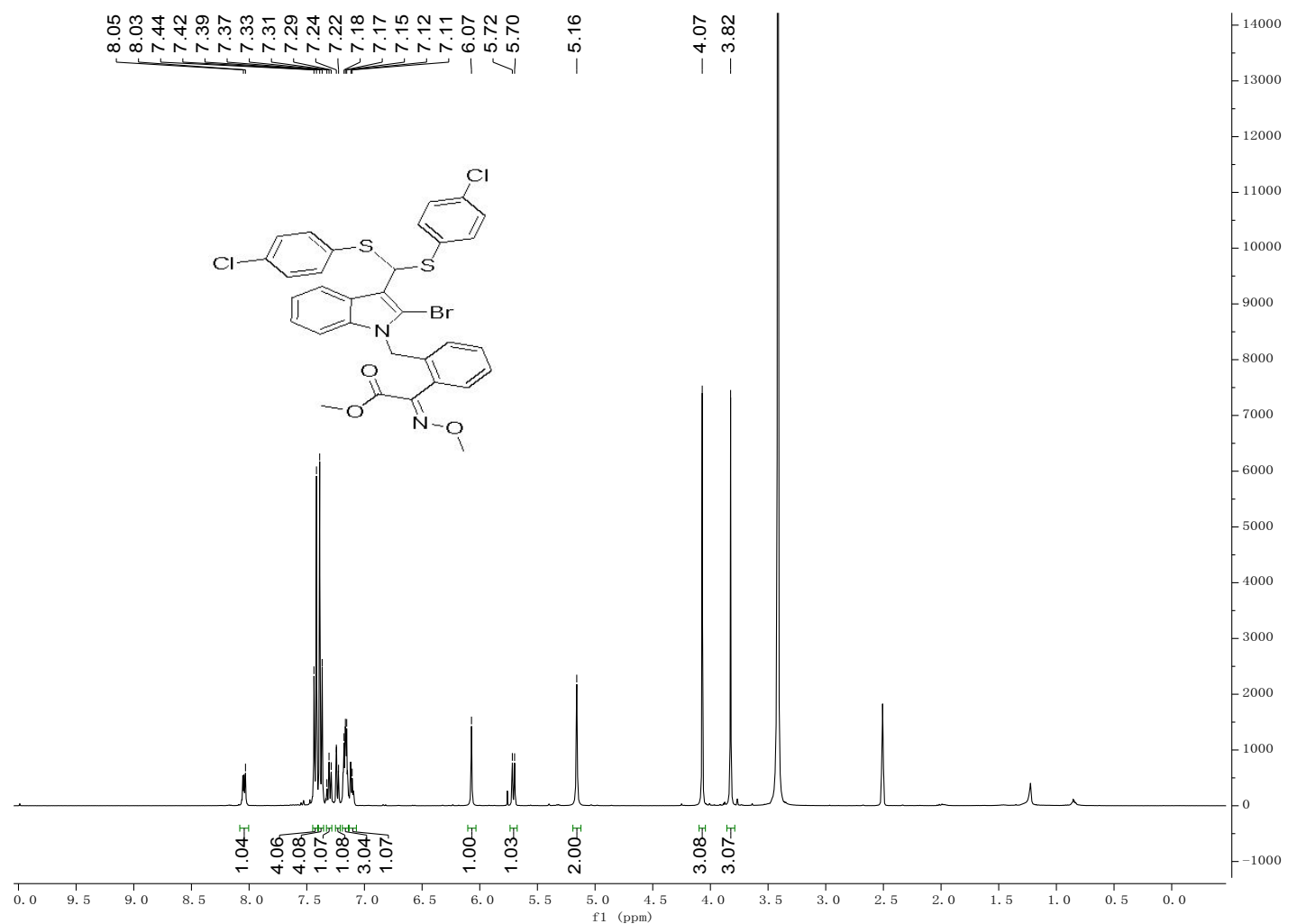

Figure 51. ${ }^{1} \mathrm{H}$ NMR ((400 MHz, DMSO- $\left.d_{6}\right)$ spectrum of compound D14. 


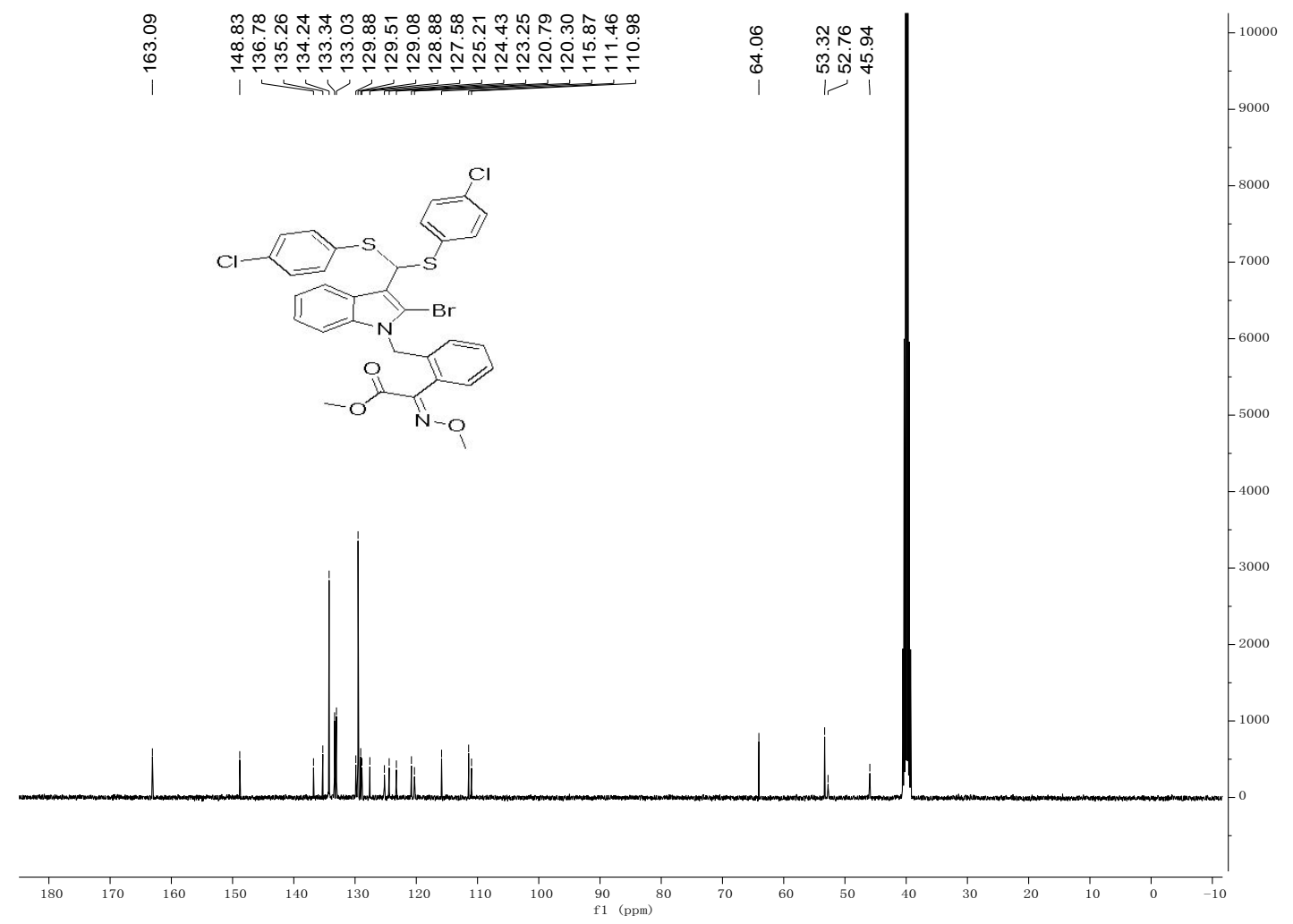

Figure 52. ${ }^{13} \mathrm{C}$ NMR $\left(100 \mathrm{MHz}\right.$, DMSO- $\left.d_{6}\right)$ spectrum of compound D14.

201904125e \#115 Rा: 1.11 AV: 1 N: 1.74E T: FIMS+ pESI Full ms [100.0000-1000.0000]

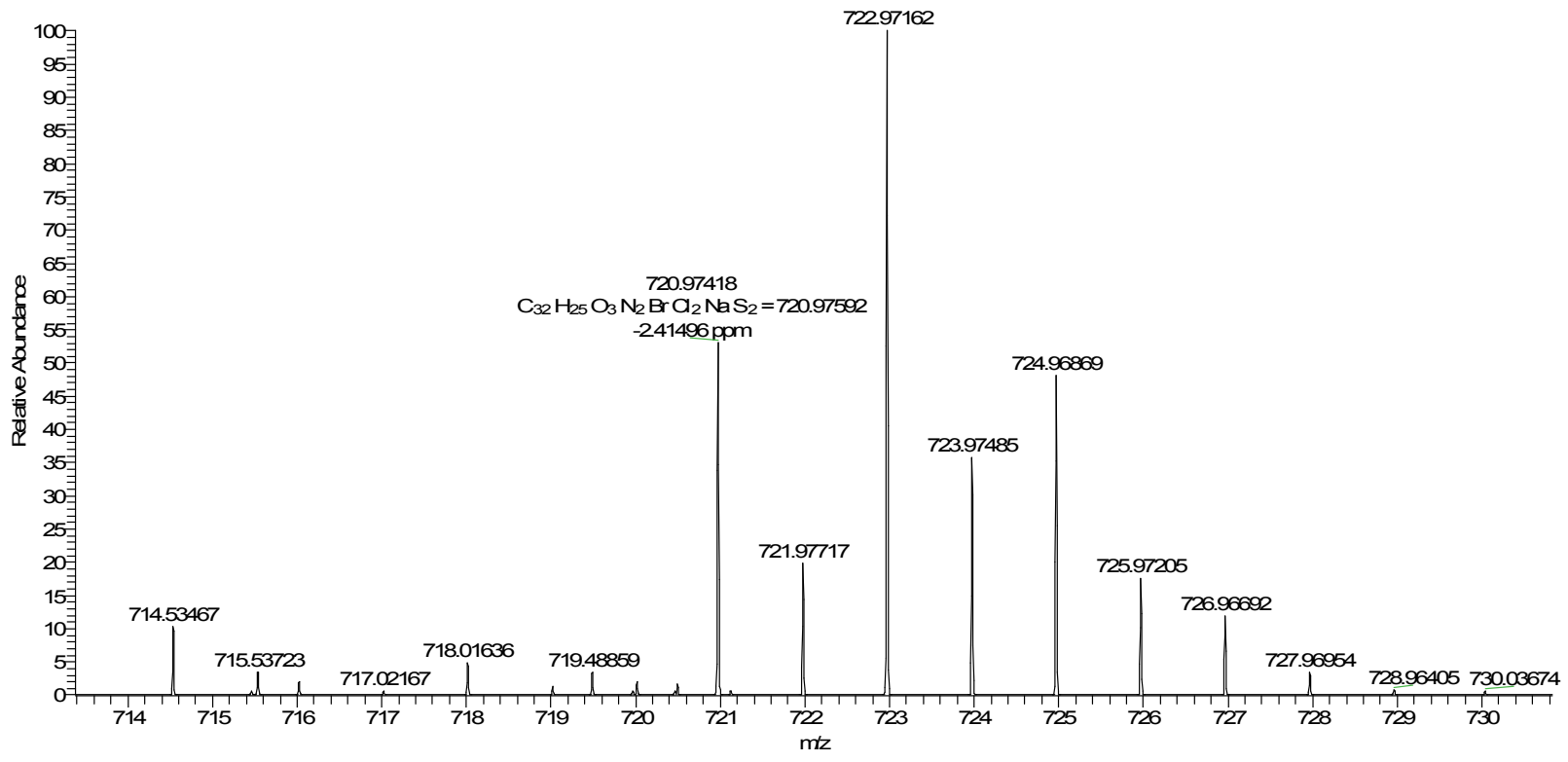

Figure 53. HRMS of compound D14. 

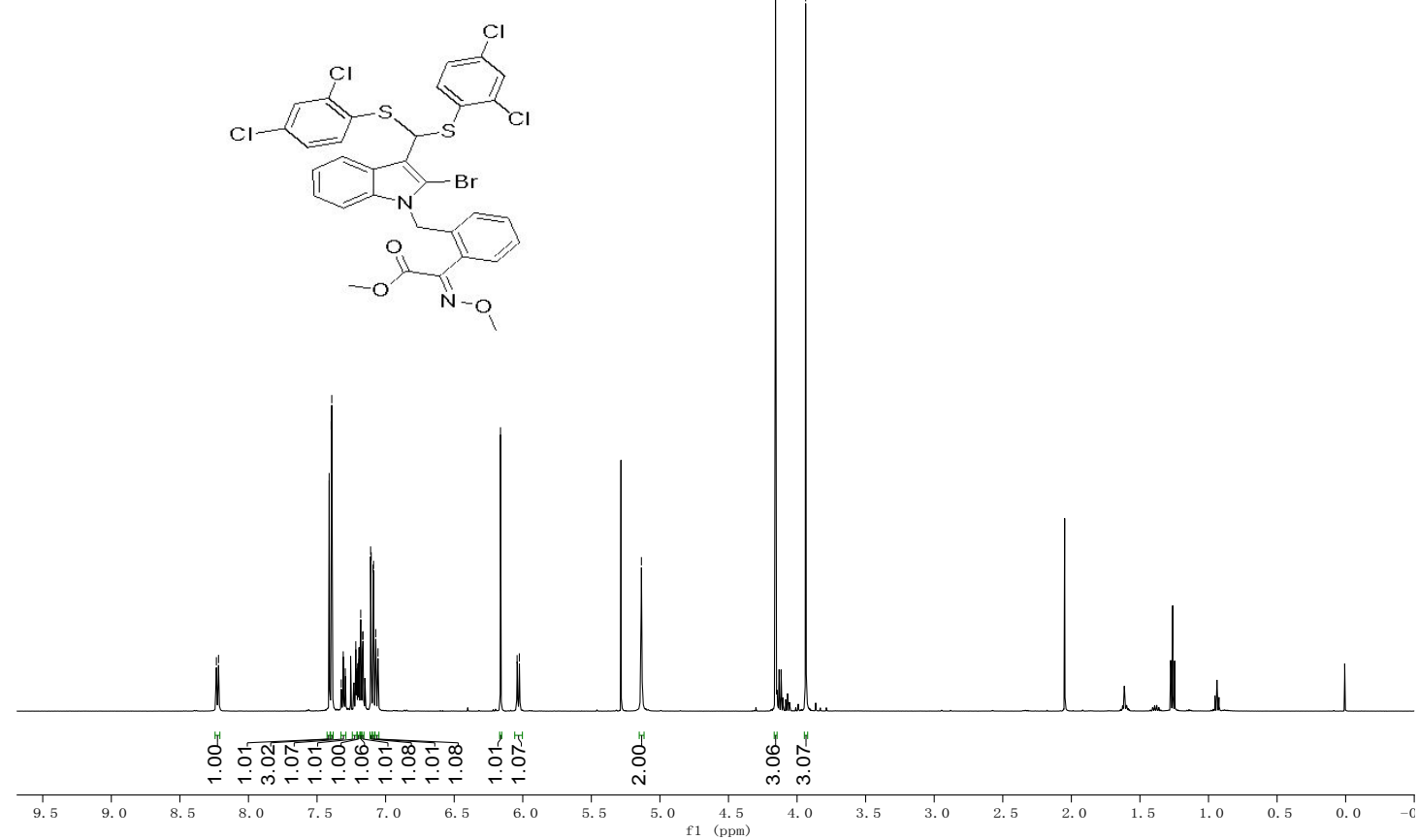

Figure 54. ${ }^{1} \mathrm{H}$ NMR ((400 MHz, DMSO- $\left.d_{6}\right)$ spectrum of compound D15.

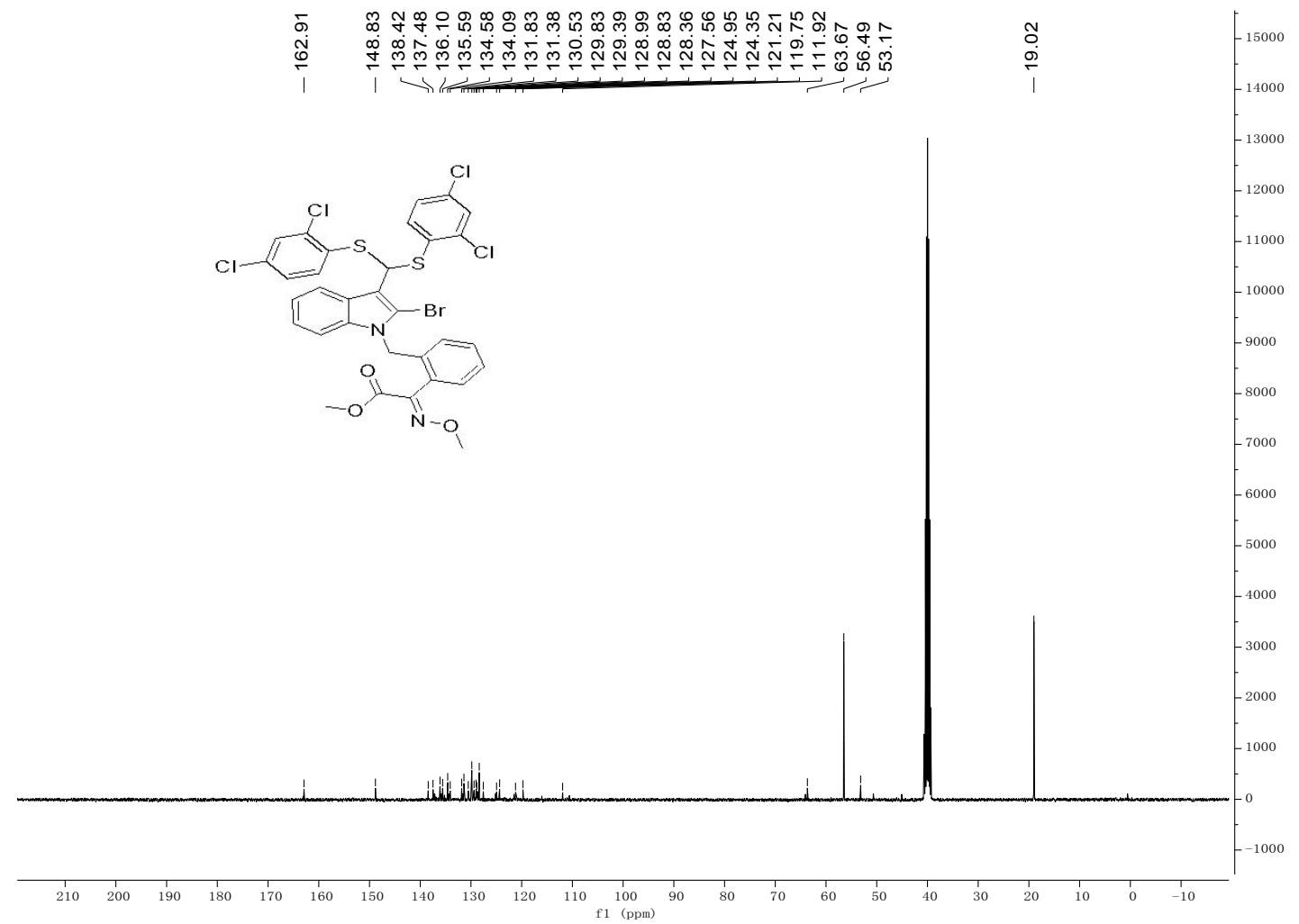

Figure 55. ${ }^{13} \mathrm{C}$ NMR (100 MHz, DMSO- $\left.d_{6}\right)$ spectrum of compound D15. 


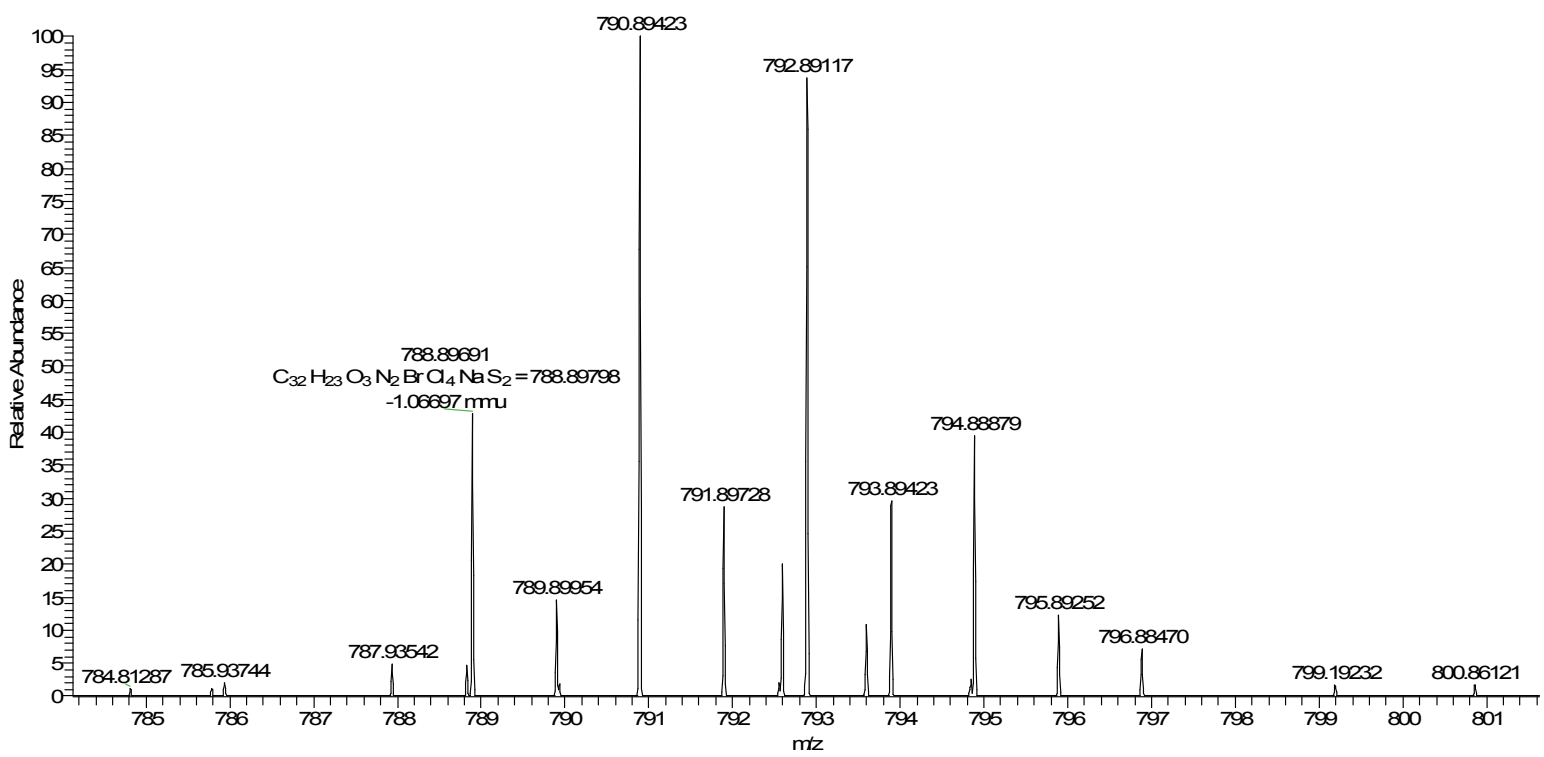

Figure 56. HRMS of compound D15.

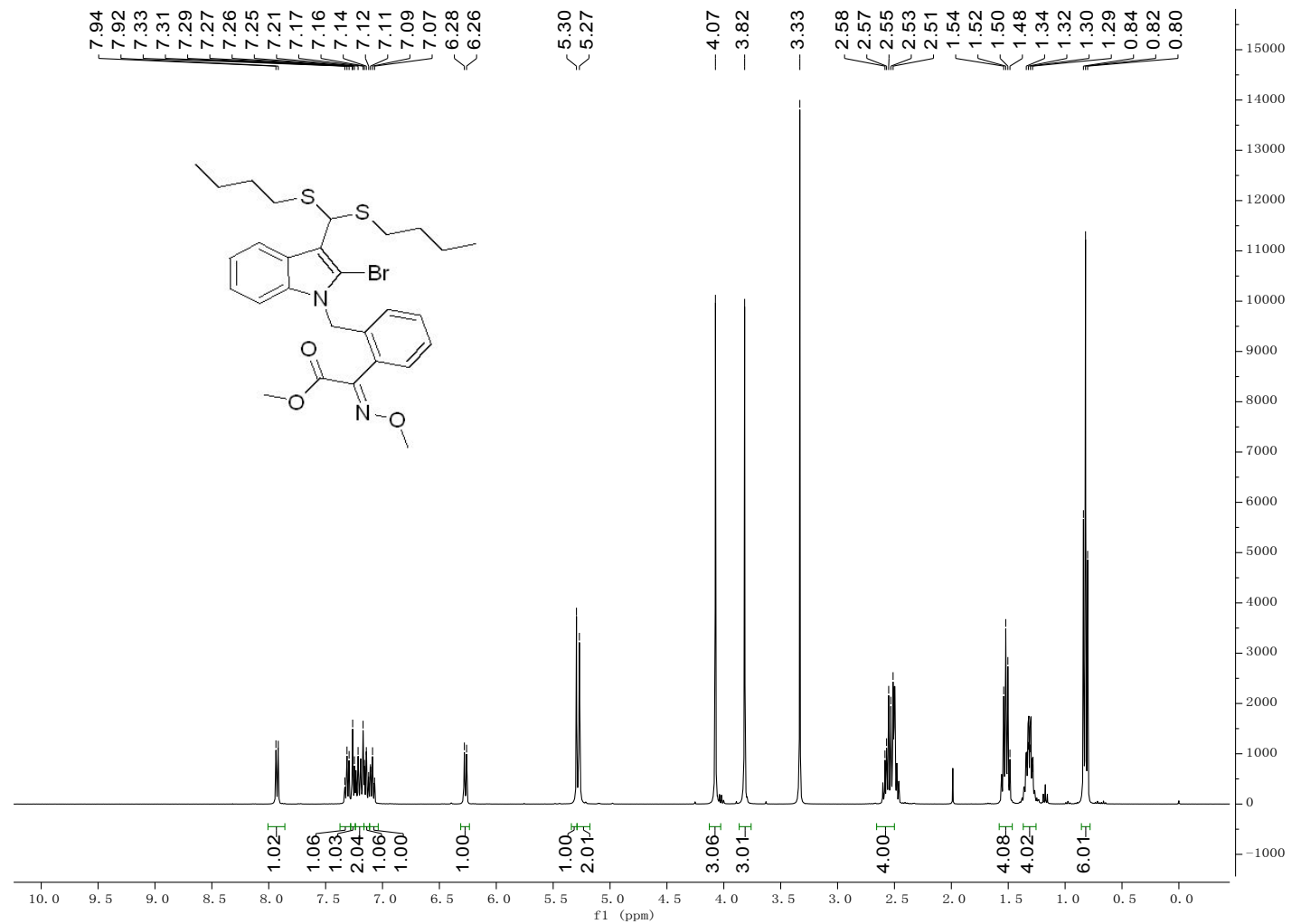

Figure 57. ${ }^{1} \mathrm{H}$ NMR ((400 MHz, DMSO- $\left.d_{6}\right)$ spectrum of compound D16. 


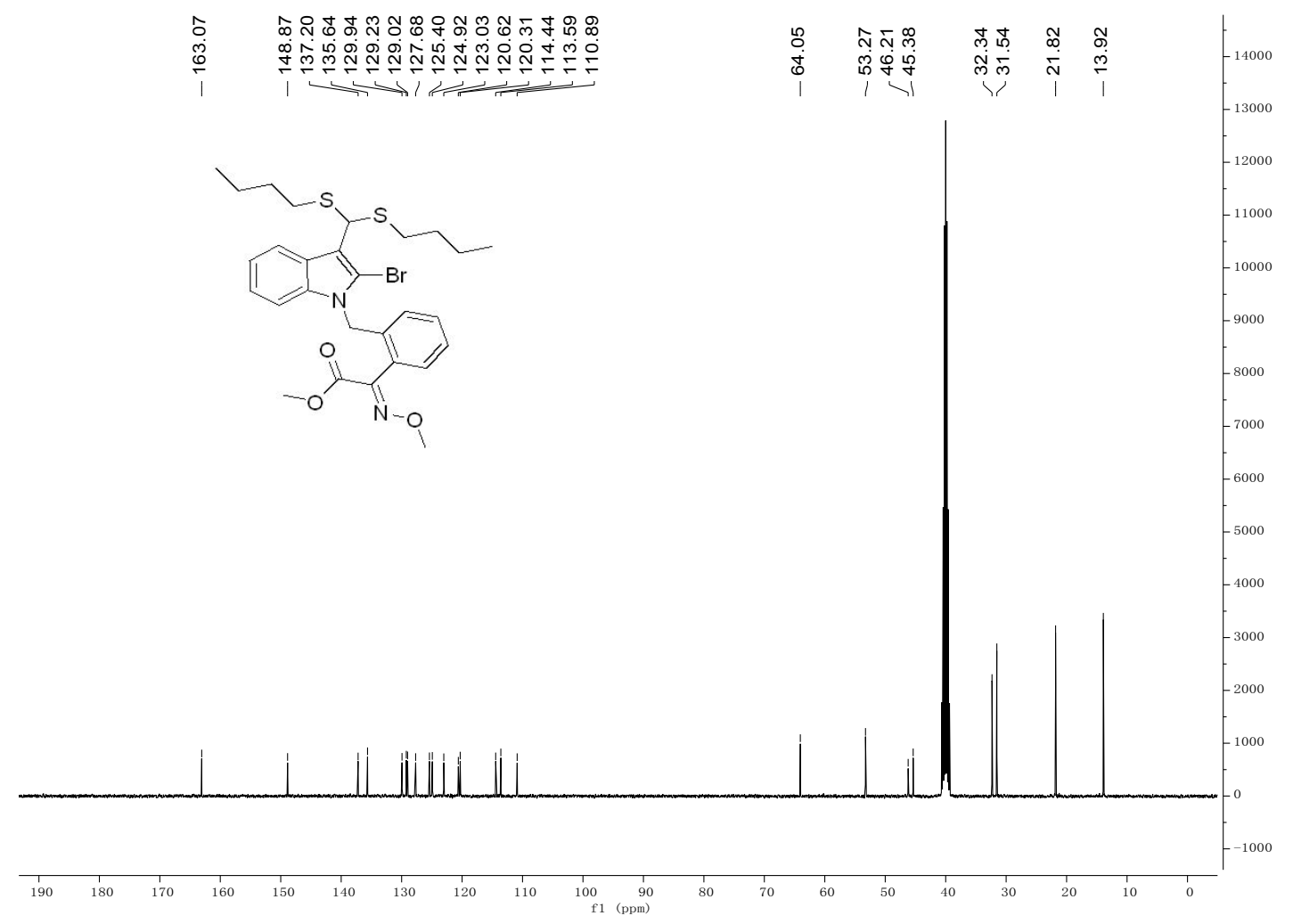

Figure 58. ${ }^{13} \mathrm{C}$ NMR $\left(100 \mathrm{MHz}\right.$, DMSO- $\left.d_{6}\right)$ spectrum of compound D16.

201904126C \#115 Rा: 1.10 AV: 1 N-238Et T: FIMS + pESI Full ms [100.0000-1000.0000]

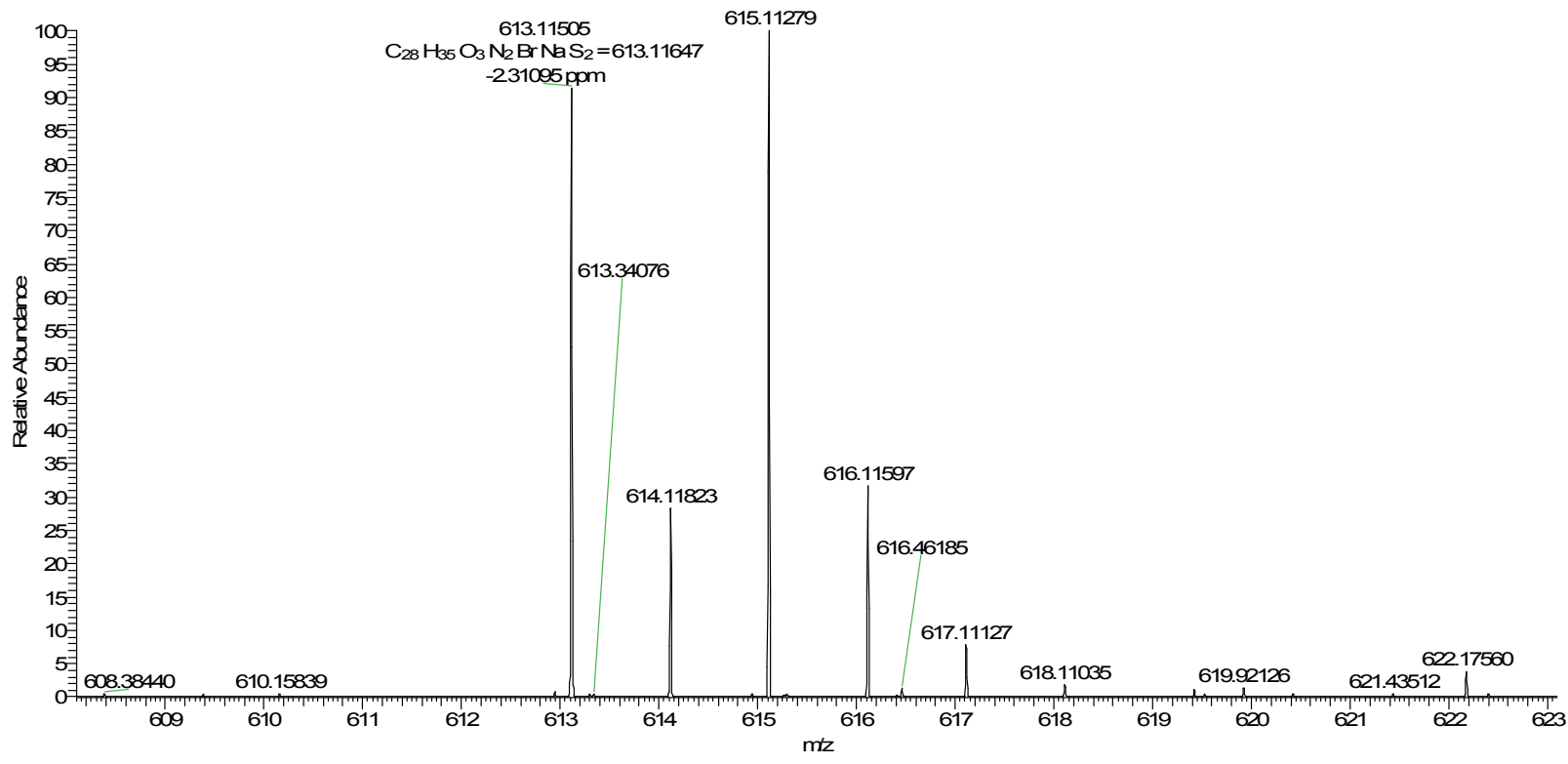

Figure 59. HRMS of compound D16. 

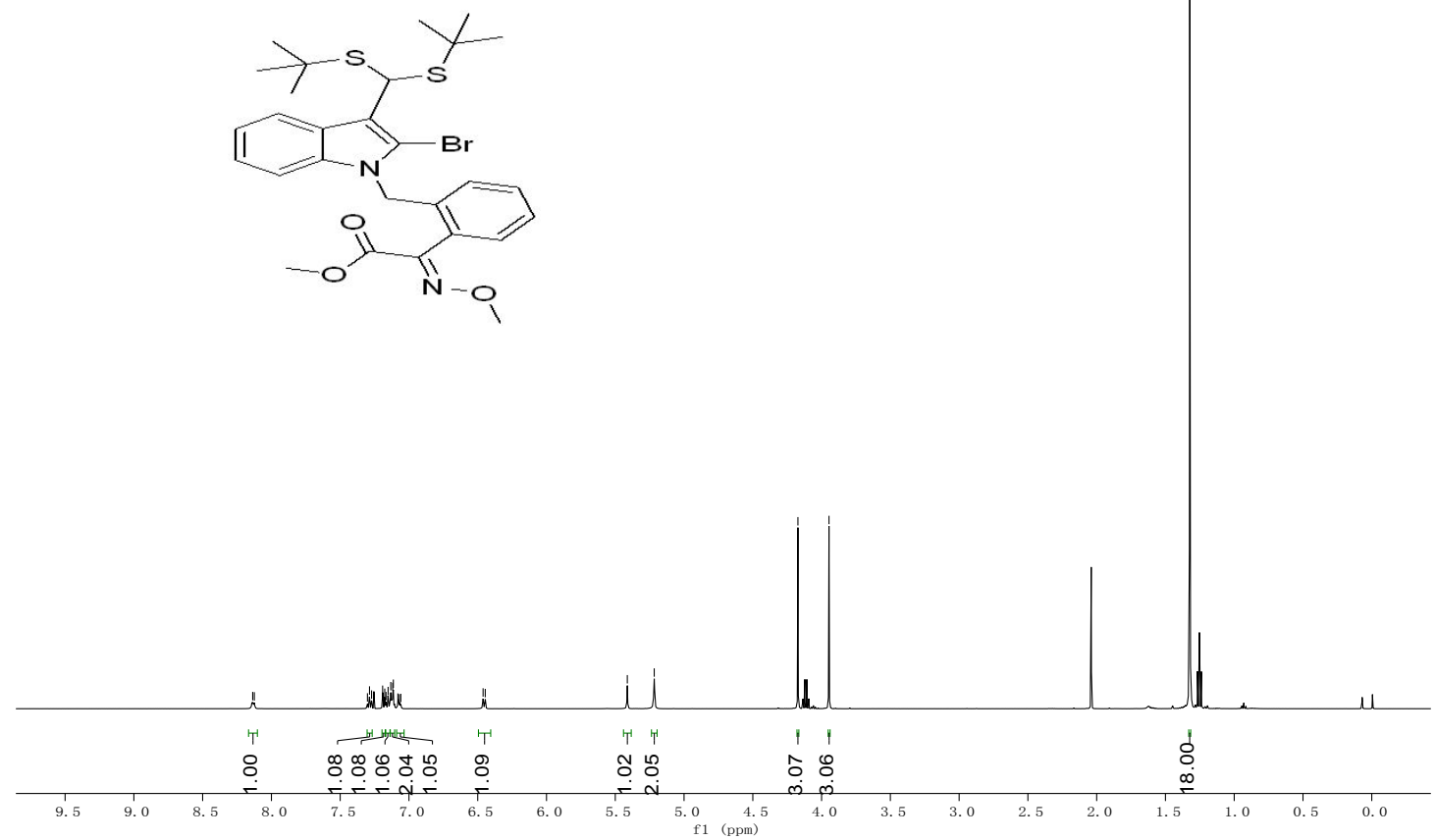

Figure 60. ${ }^{1} \mathrm{H}$ NMR ((400 MHz, DMSO- $\left.d_{6}\right)$ spectrum of compound D17.

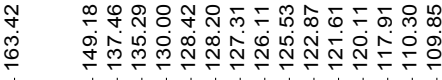

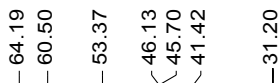<smiles>CO/N=C(/C(=O)OC)c1ccccc1Cn1c(Br)c(C(SC(C)(C)C)SC(C)(C)C)c2ccccc21</smiles>

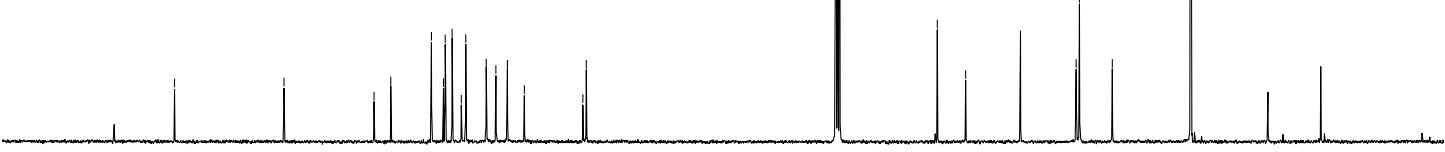

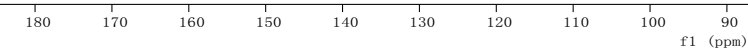

Figure 61. ${ }^{13} \mathrm{C}$ NMR $\left(100 \mathrm{MHz}, \mathrm{DMSO}-d_{6}\right)$ spectrum of compound D17. 
2018093011 \#53 Rा: 0.51 AV: 1 N. 1.44 ES
T: FIMS + pESIFull ms [100.0000-1000.0000]

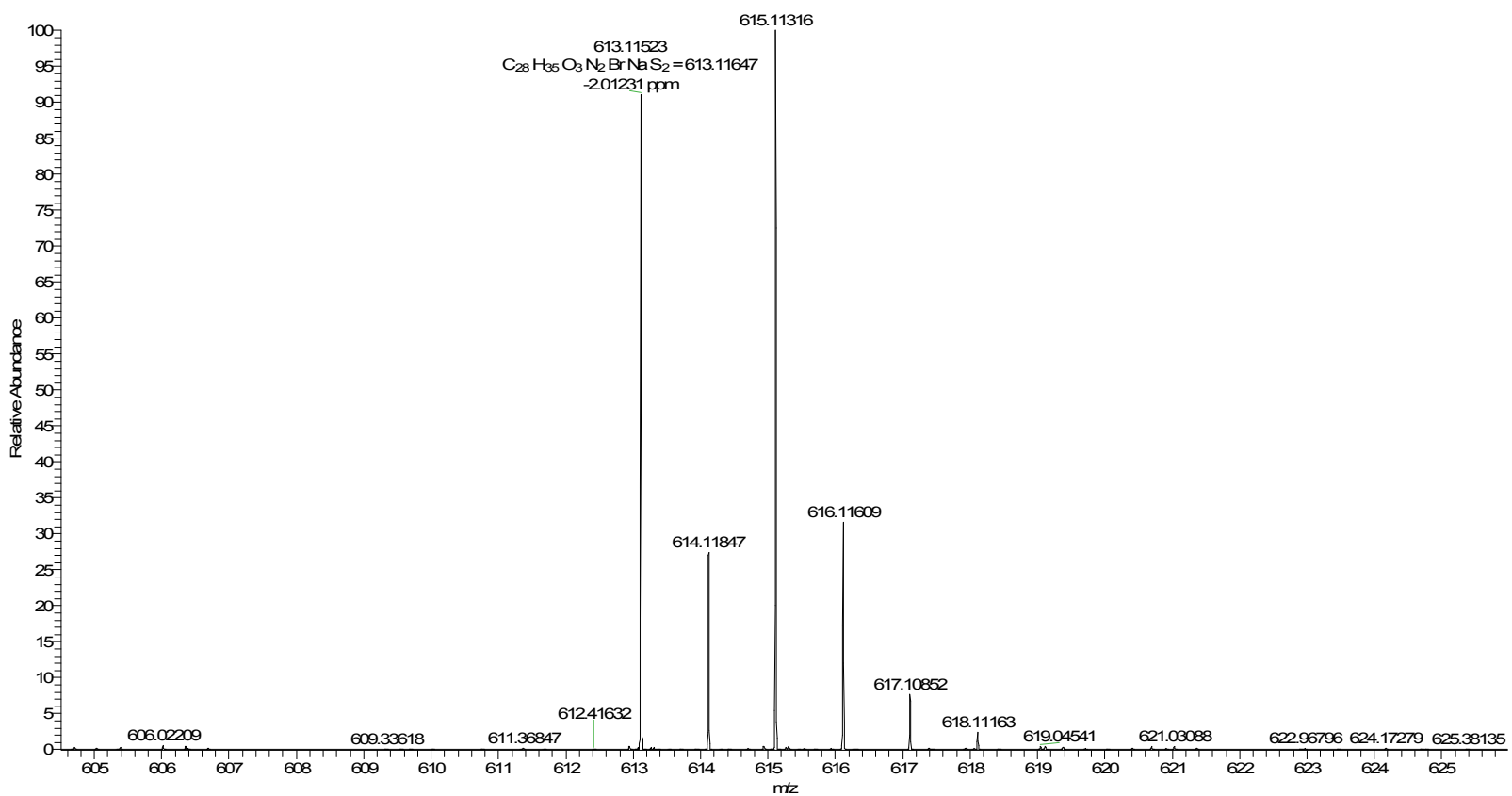

Figure 62. HRMS of compound D17.

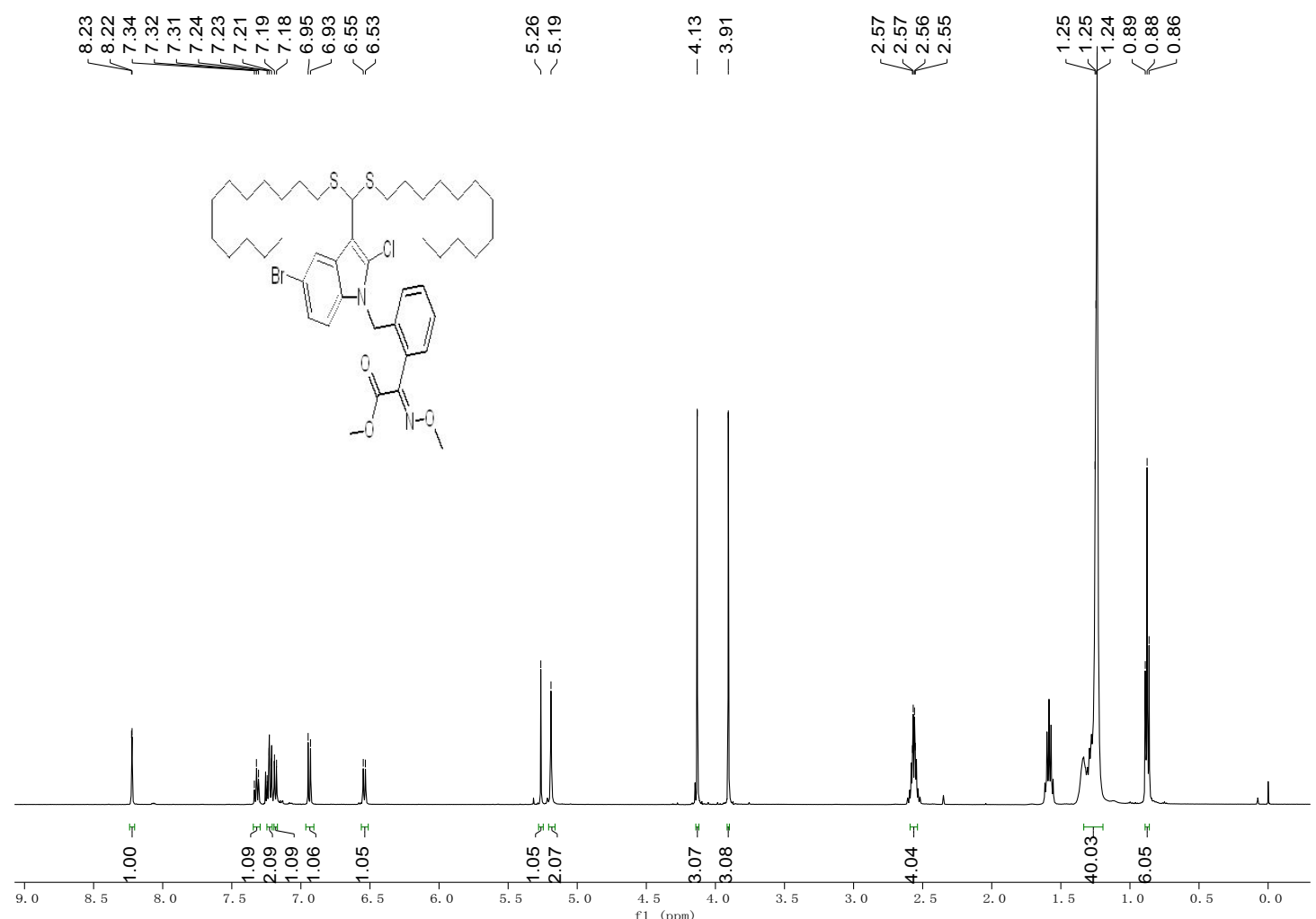

Figure 63. ${ }^{1} \mathrm{H}$ NMR $\left(\left(400 \mathrm{MHz}, \mathrm{DMSO}-d_{6}\right)\right.$ spectrum of compound D18. 

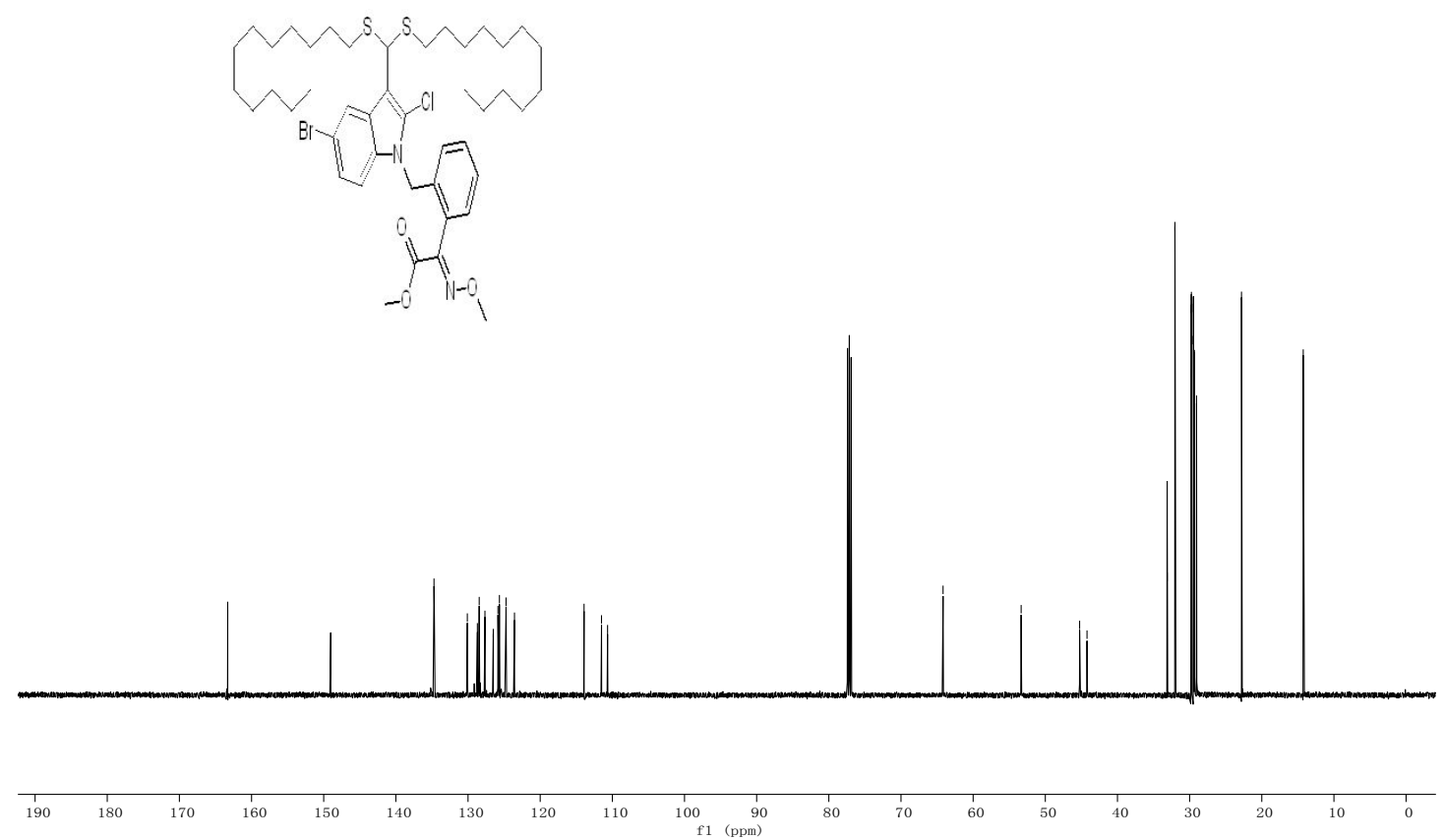

Figure 64. ${ }^{13} \mathrm{C}$ NMR (100 MHz, DMSO- $d_{6}$ ) spectrum of compound D18.

142 \#56 Rा: 0.54 AV: 1 N: 1.34E4 T: FIMS-pESI Full ms [100.0000-1000.0000]

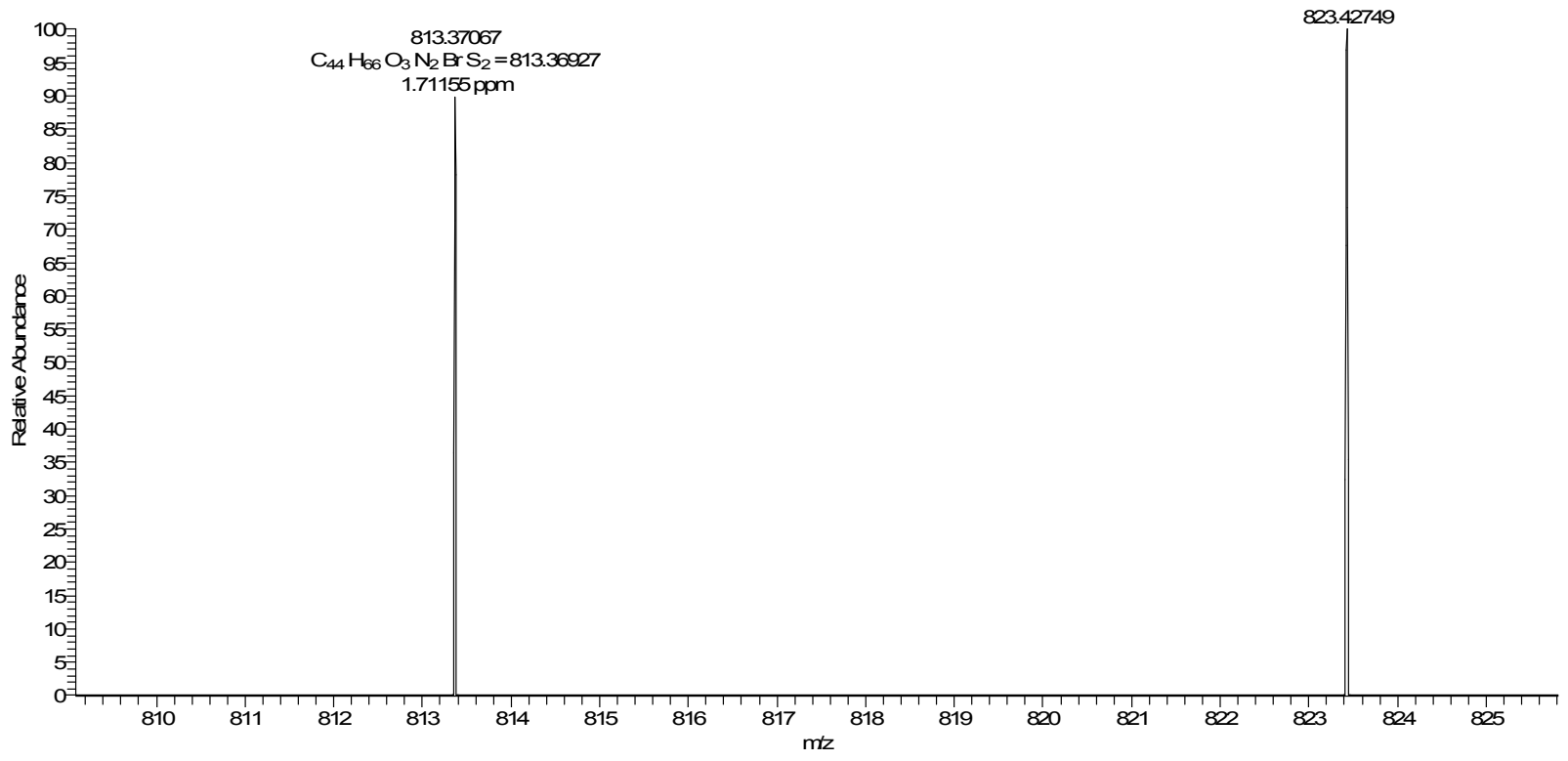

Figure 65. HRMS of compound D18. 


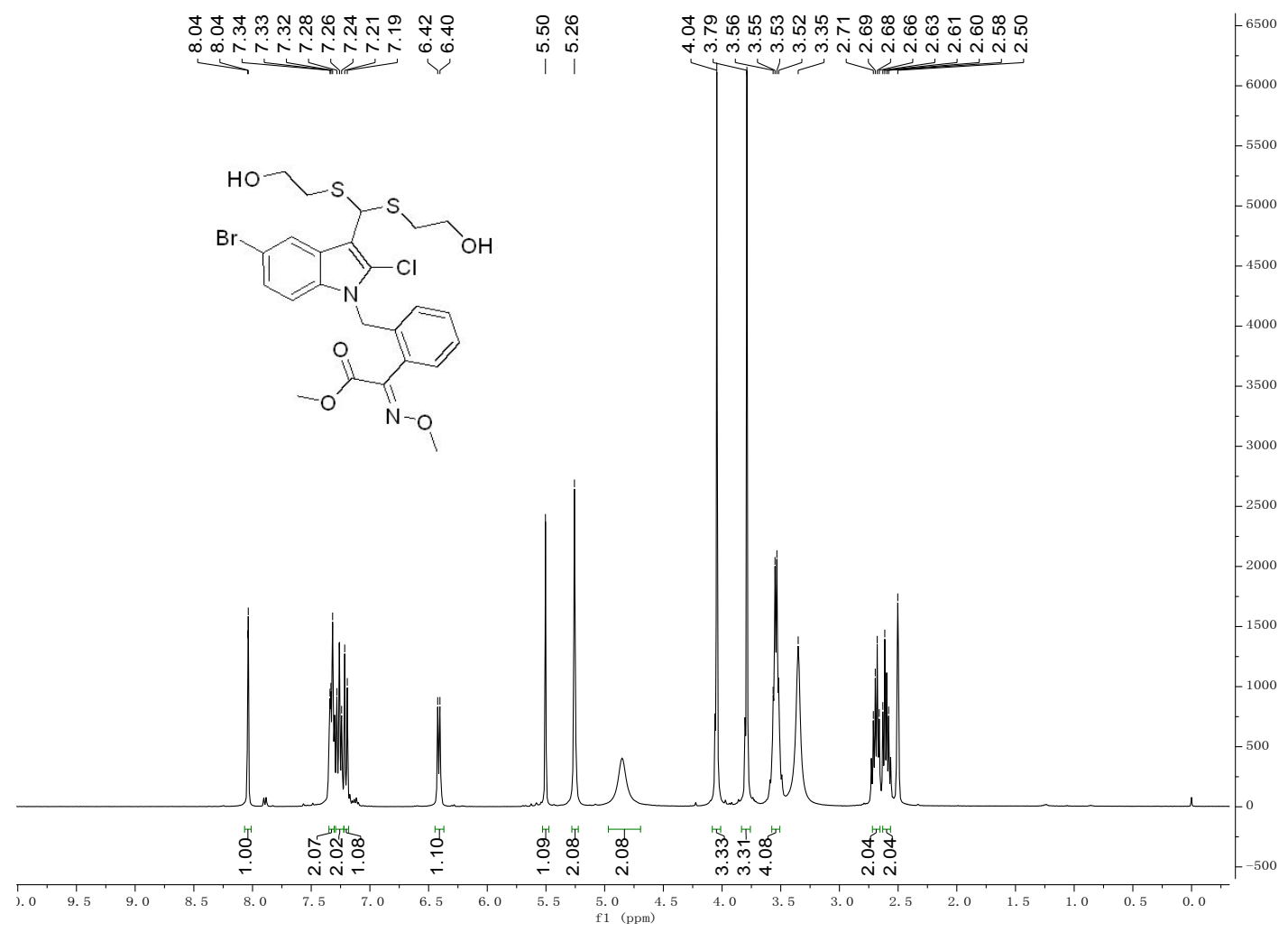

Figure 66. ${ }^{1} \mathrm{H}$ NMR ((400 MHz, DMSO- $\left.d_{6}\right)$ spectrum of compound D19.

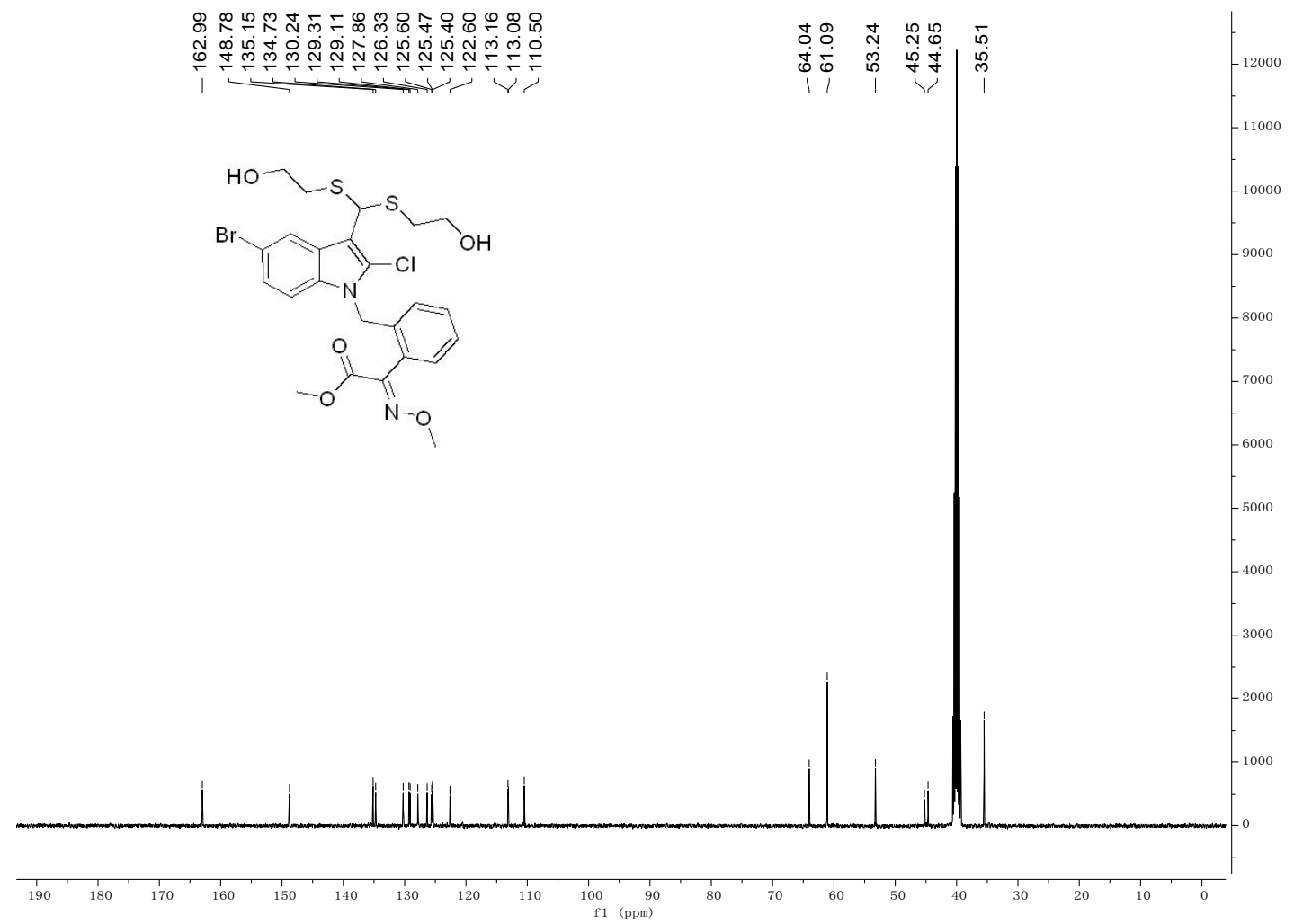

Figure 67. ${ }^{13} \mathrm{C}$ NMR (100 MHz, DMSO- $\left.d_{6}\right)$ spectrum of compound D19. 


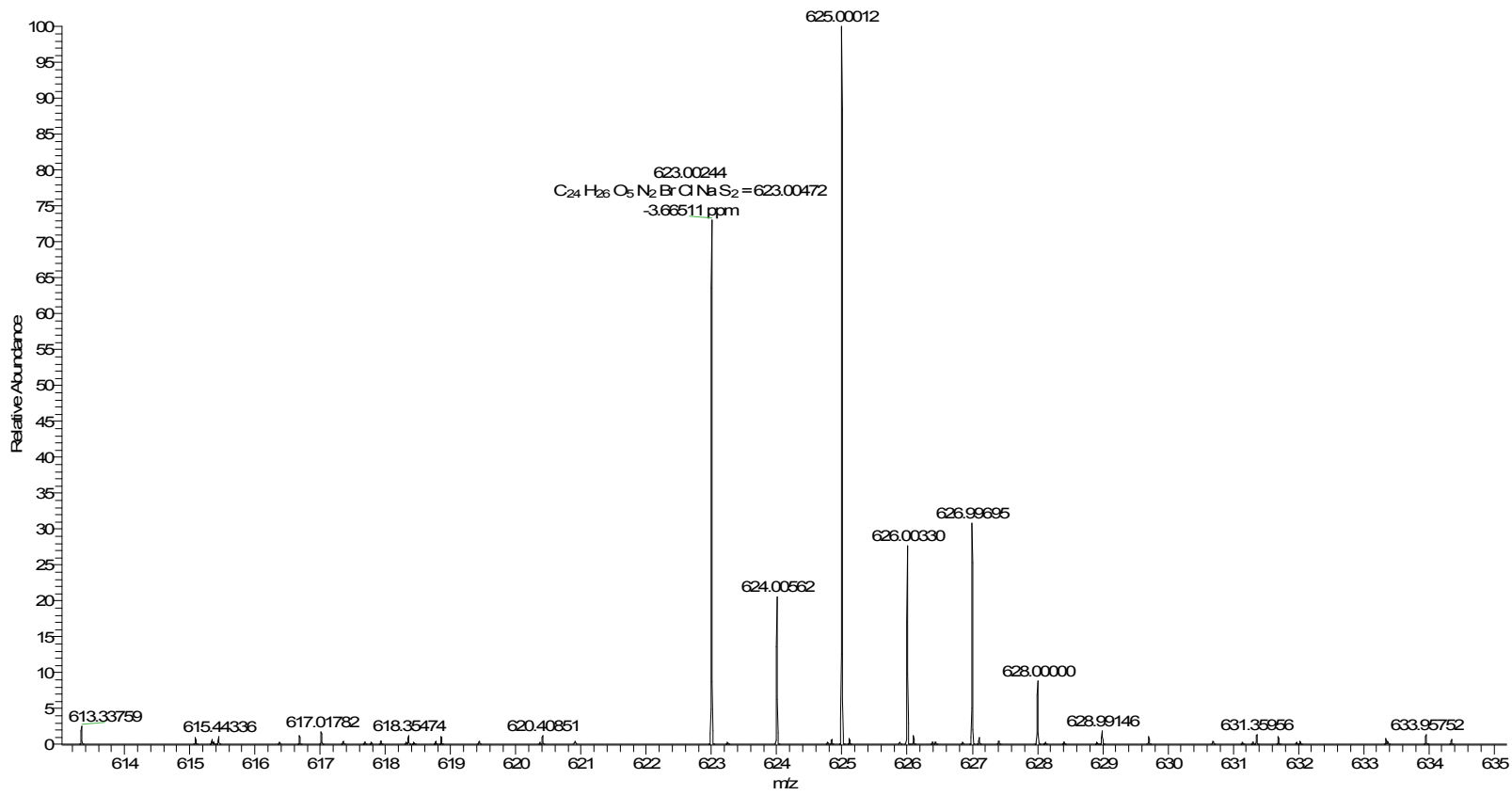

Figure 68. HRMS of compound D19.

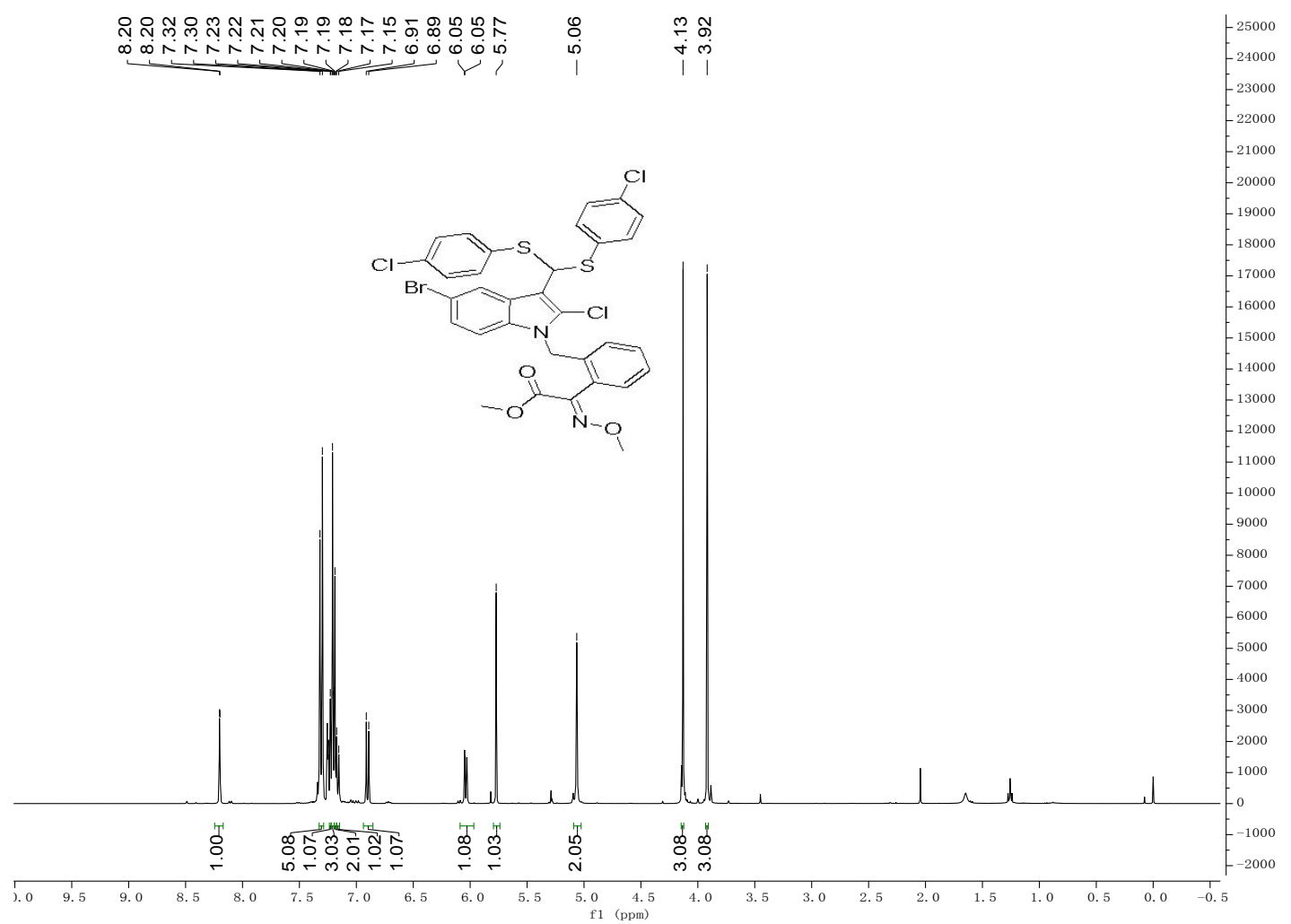

Figure 69. ${ }^{1} \mathrm{H}$ NMR $\left(\left(400 \mathrm{MHz}, \mathrm{DMSO}-d_{6}\right)\right.$ spectrum of compound D20. 


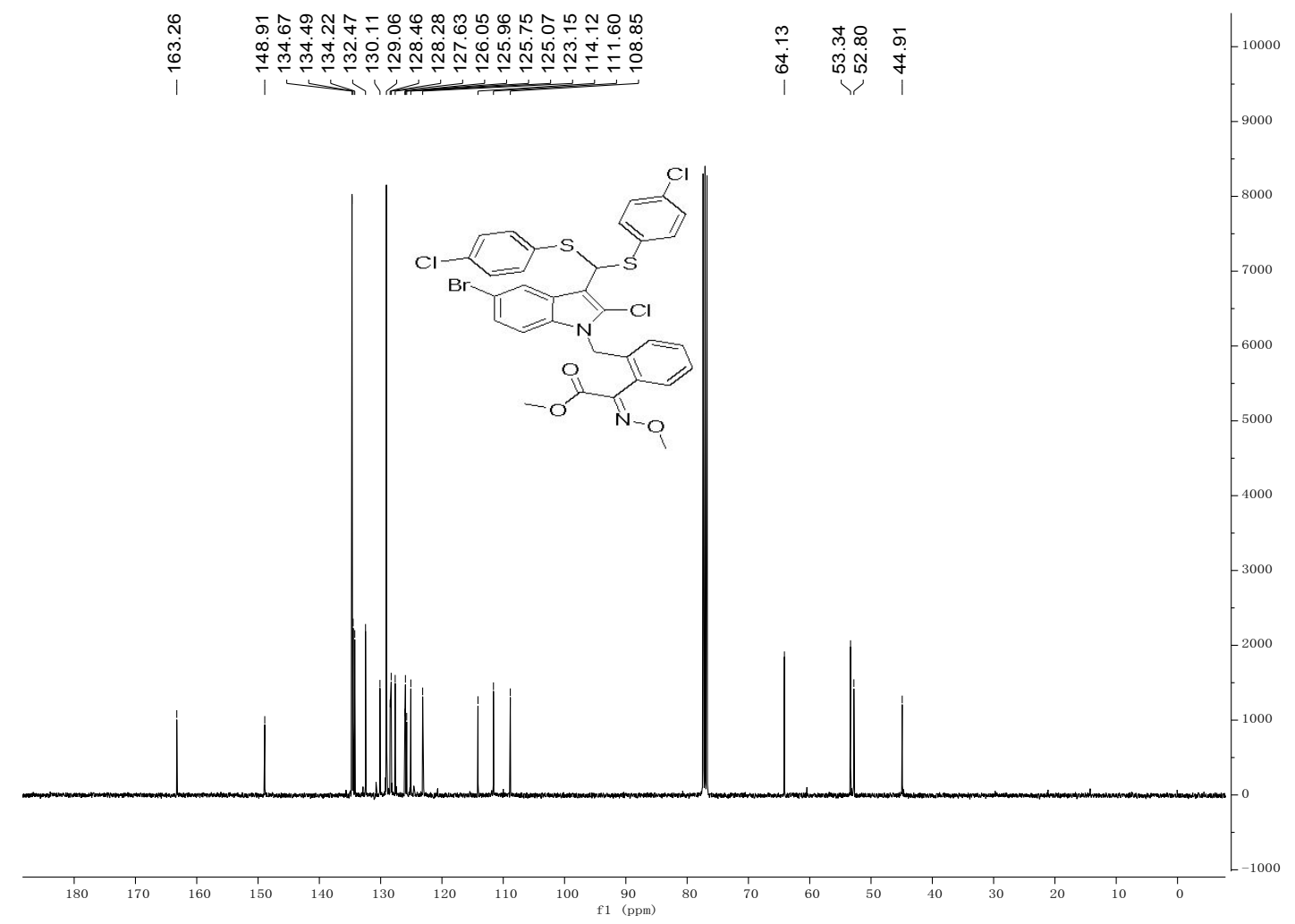

Figure 70. ${ }^{13} \mathrm{C}$ NMR $\left(100 \mathrm{MHz}, \mathrm{DMSO}-d_{6}\right)$ spectrum of compound D20.

201904171 \#161 Rा: 1.55 AV: $1 \quad N \cdot 4.13 E]$

T: FIMS + pESI Full ms [100.0000-1000.0000]

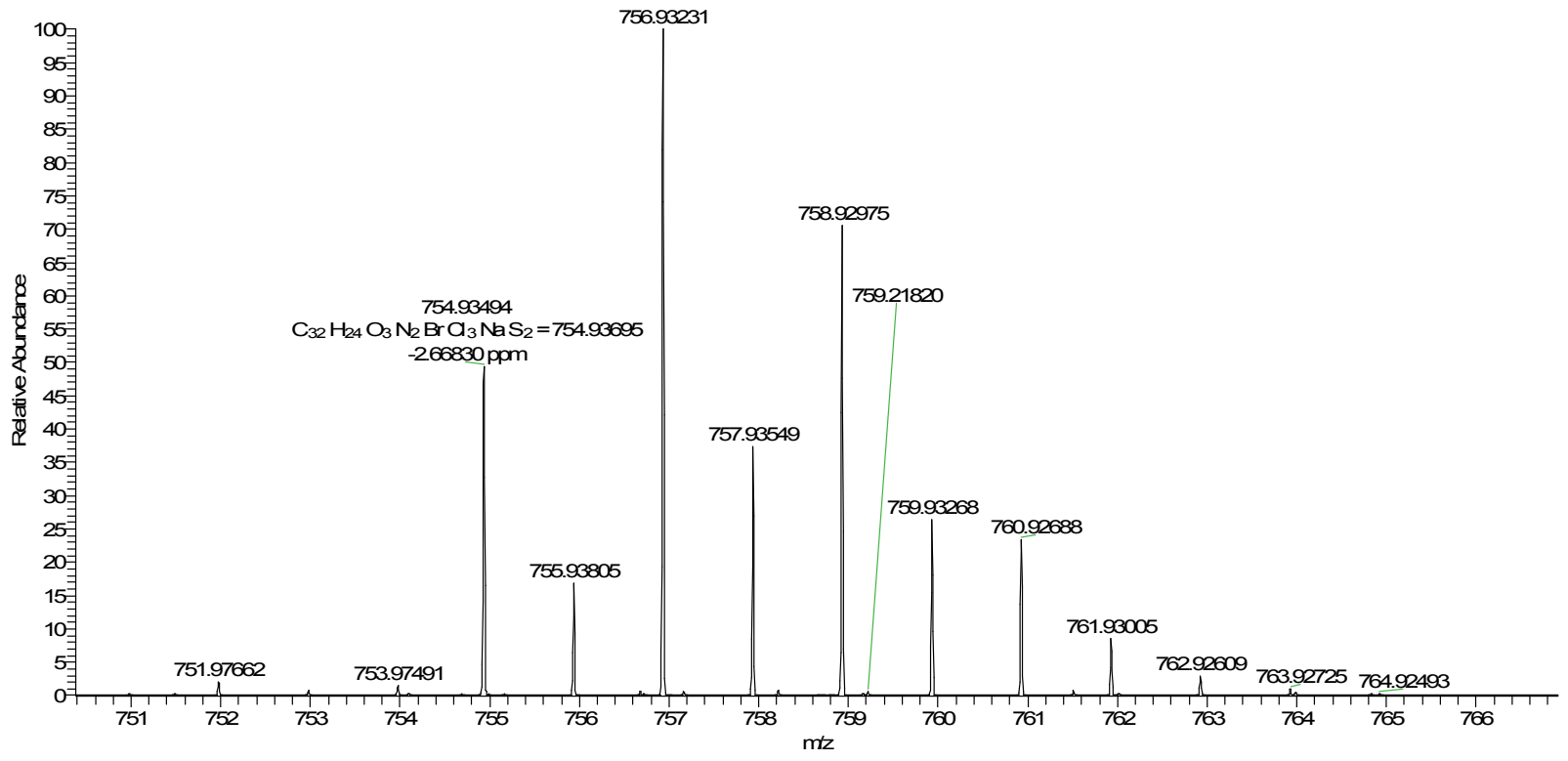

Figure 71 HRMS of compound D20. 


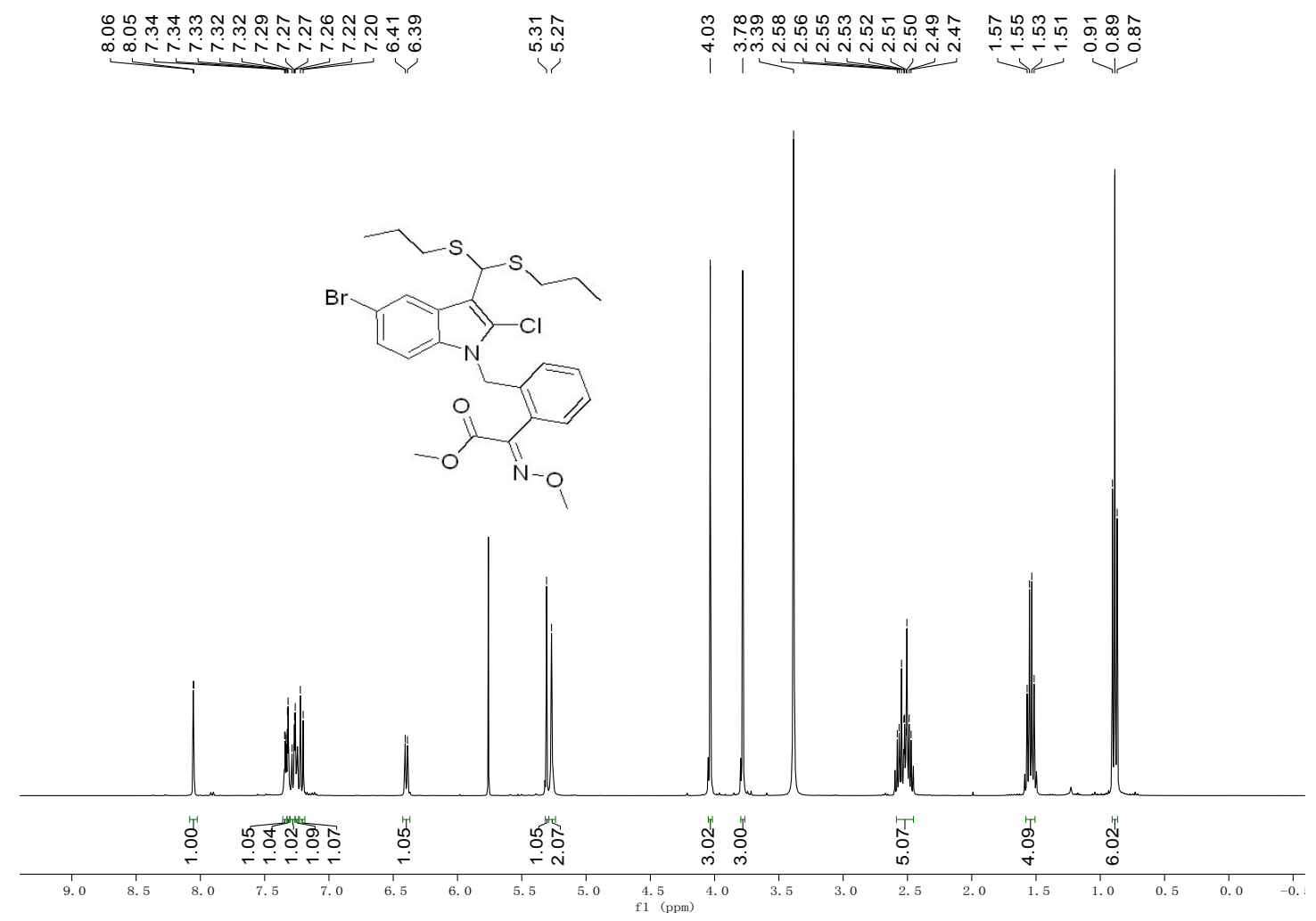

Figure 72. ${ }^{1} \mathrm{H}$ NMR ((400 MHz, DMSO- $\left.d_{6}\right)$ spectrum of compound D21.
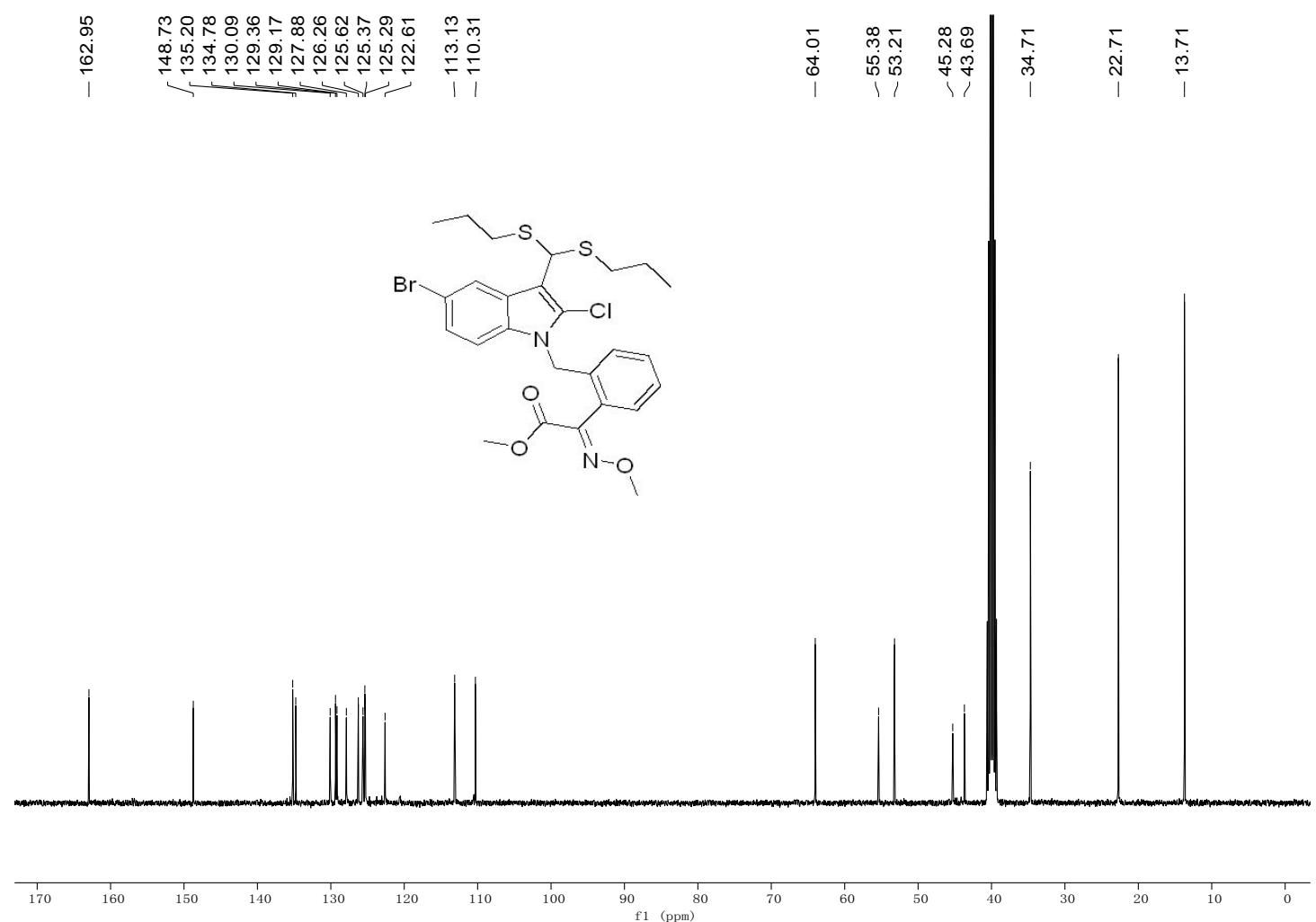

Figure 73. ${ }^{13} \mathrm{C}$ NMR (100 MHz, DMSO- $d_{6}$ ) spectrum of compound D21. 
2019041718\#105 RT: 1.01 AV: 1 N- 6.72E]
T: FIMS+p ESI Full ms [100.0000-1000.0000]

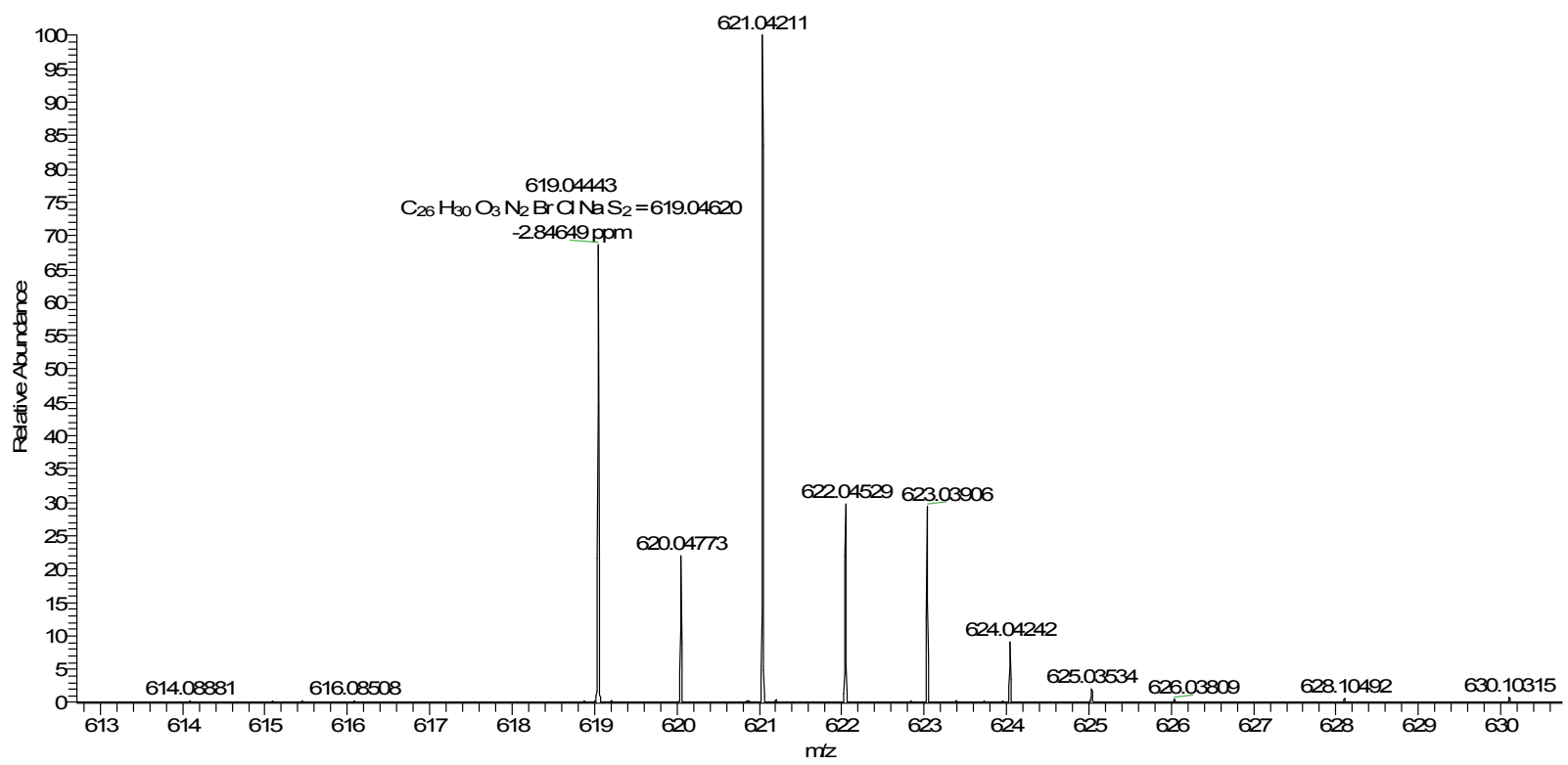

Figure 74. HRMS of compound D21.

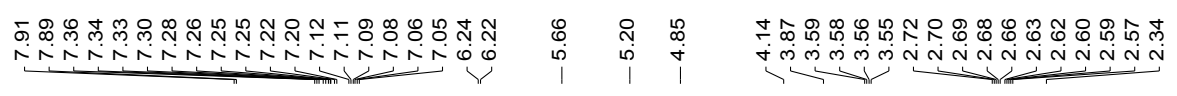

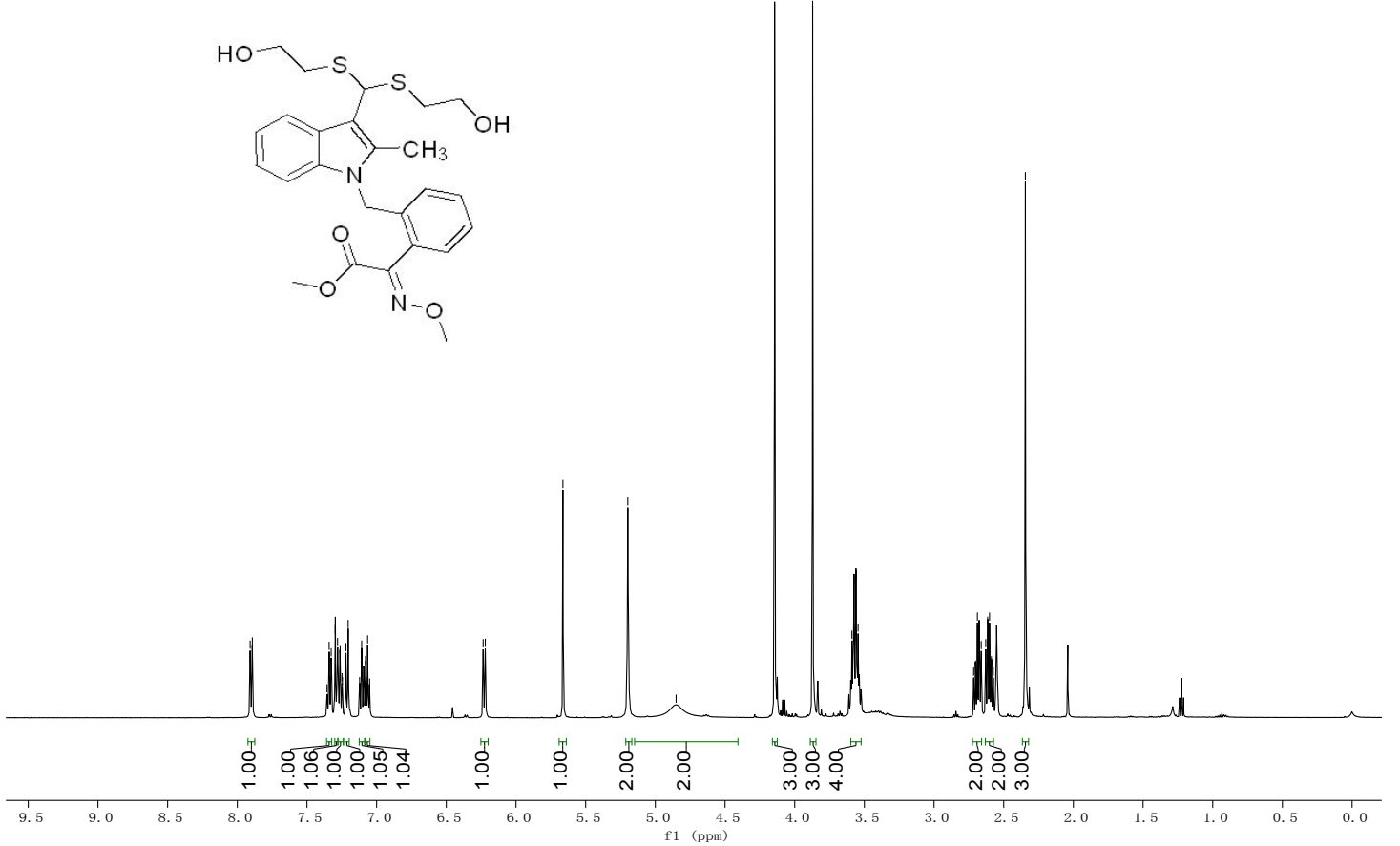

Figure 75. ${ }^{1} \mathrm{H}$ NMR ((400 MHz, DMSO- $\left.d_{6}\right)$ spectrum of compound $\mathbf{D 2 2}$.

56 


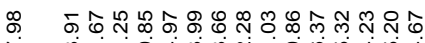

苞<smiles>CO/N=C(/C(=O)OC)c1ccccc1Cn1c(C)c(C(SCCO)SCCO)c2ccccc21</smiles>

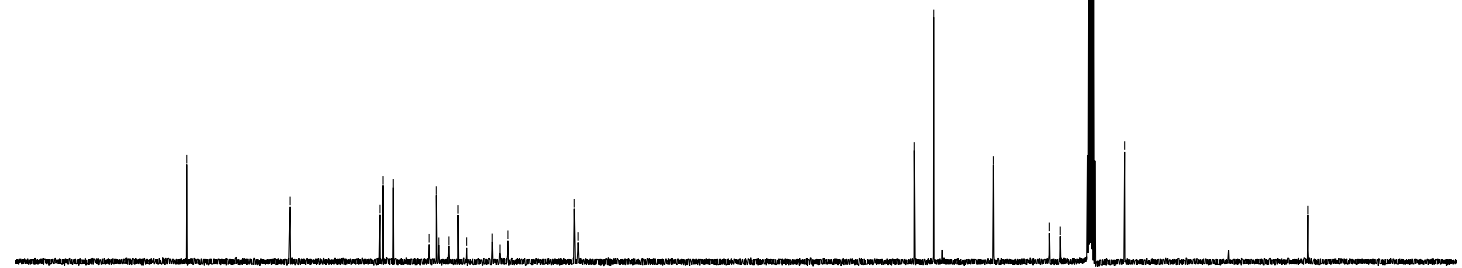

190

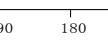

Figure 76. ${ }^{13} \mathrm{C}$ NMR ( $\left.100 \mathrm{MHz}, \mathrm{DMSO}-d_{6}\right)$ spectrum of compound D22.

201904172C \#45 Rा: 0.43 AV: 1 N: 9.51E T: FIMS+ pESI Ful ms [100.0000-1000.0000]

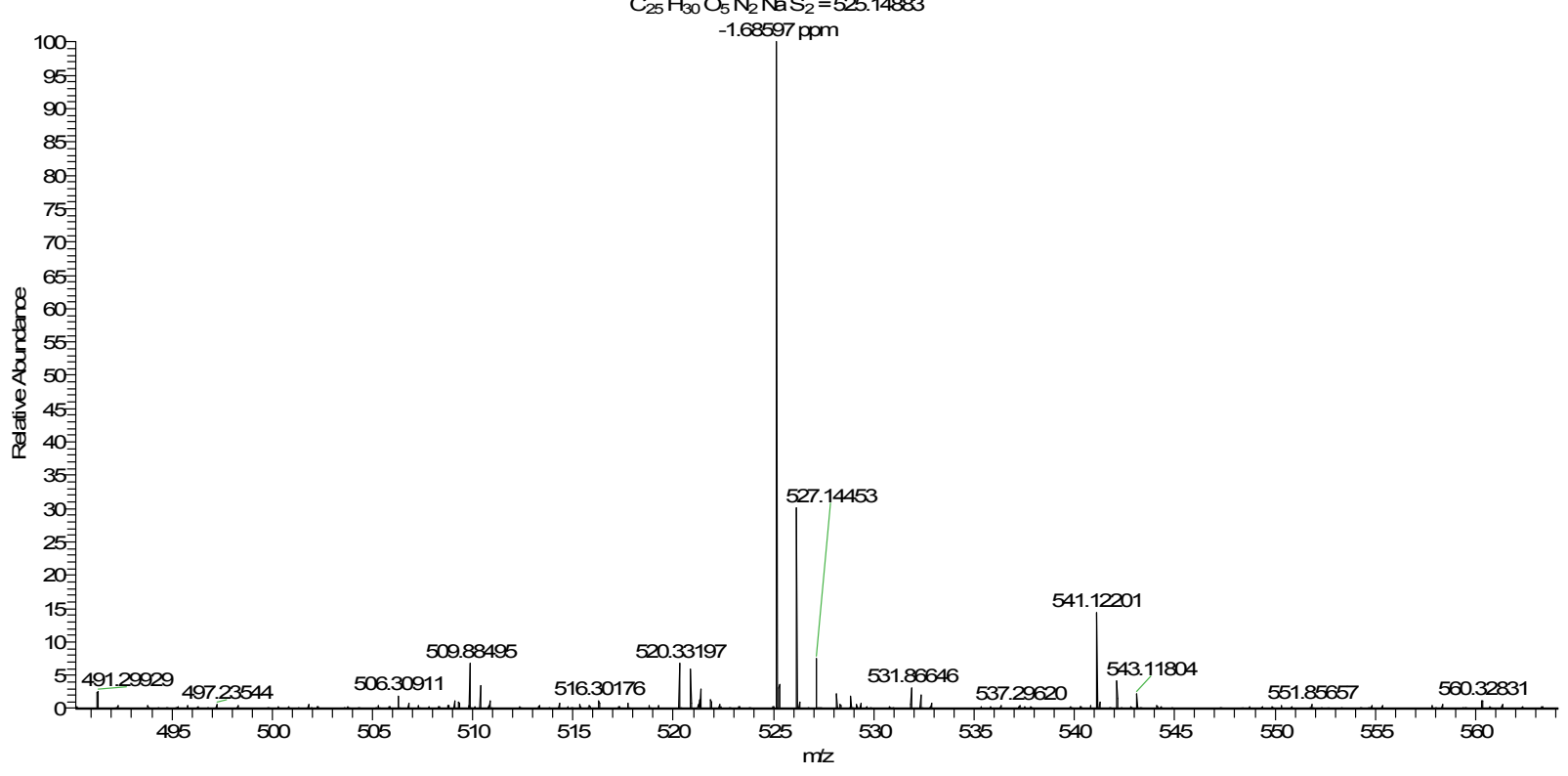

Figure 77. HRMS of compound D22. 
<smiles>CCSc1c(C)n(Cc2ccccc2/C(=N\OC)C(=O)OC)c2ccccc12</smiles>

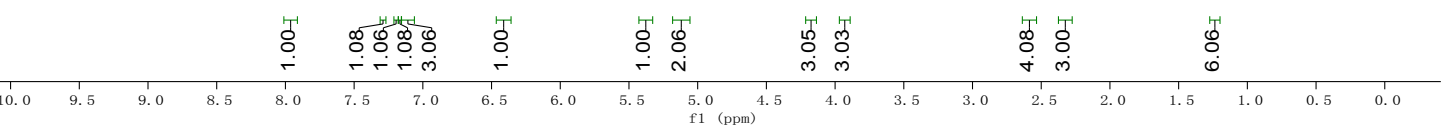

Figure 78. ${ }^{1} \mathrm{H}$ NMR ((400 MHz, DMSO- $\left.d_{6}\right)$ spectrum of compound D23.

లై

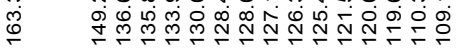

i

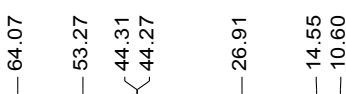<smiles>CCO/N=C(/C(=O)OC)c1ccccc1Cn1c(C)c(C(SCC)SCC)c2ccccc21</smiles>
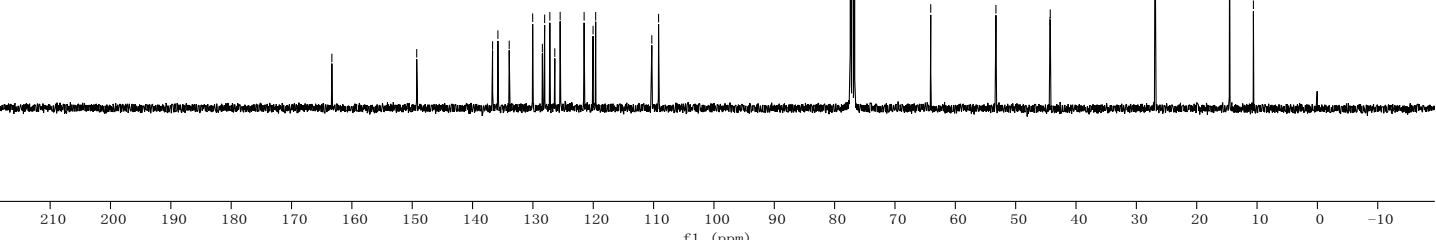

Figure 79. ${ }^{13} \mathrm{C}$ NMR (100 MHz, DMSO- $\left.d_{6}\right)$ spectrum of compound D23. 
2019041721 \#67 Rा: 0.64 AV: 1 N.225E8

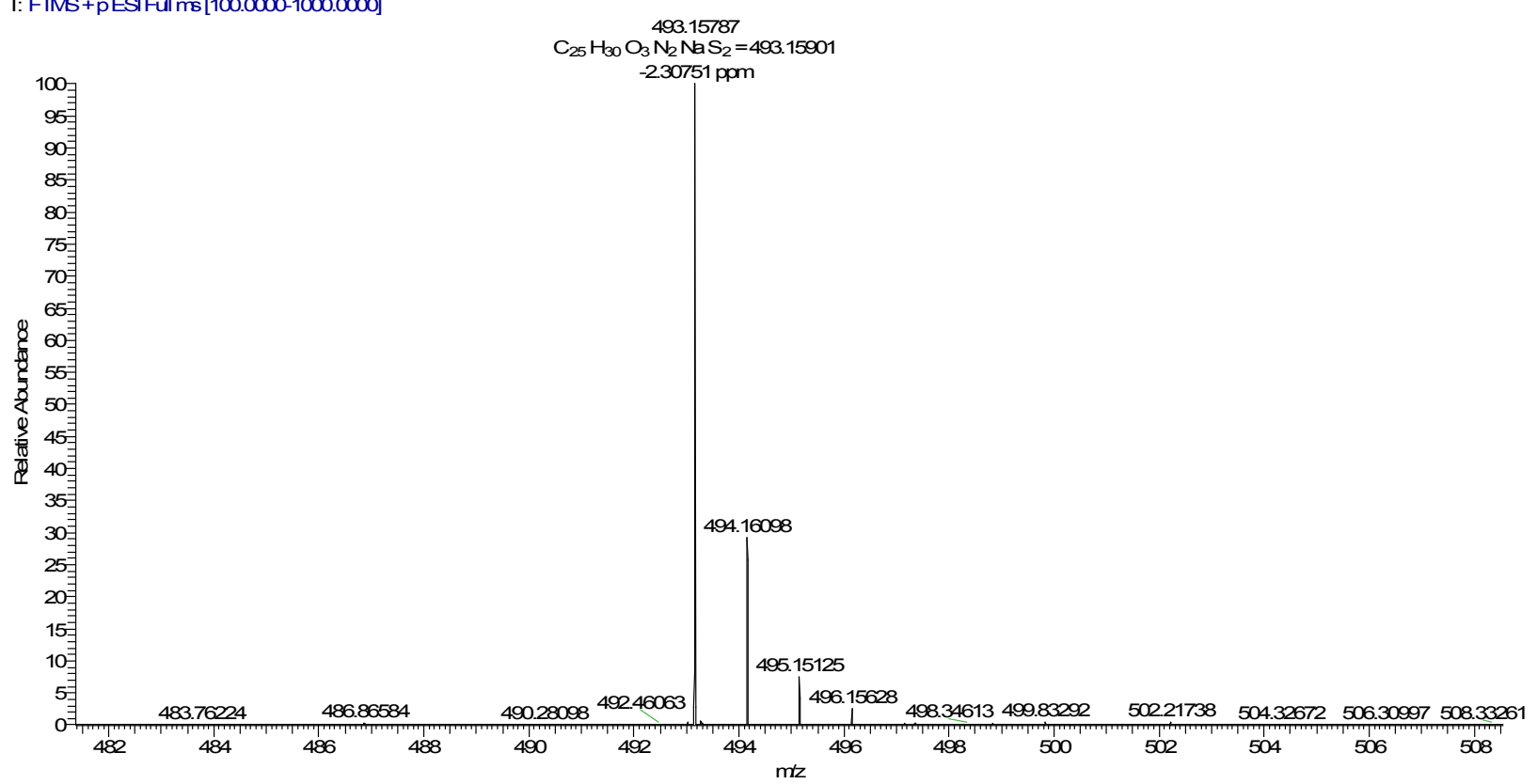

Figure 80. HRMS of compound D23.

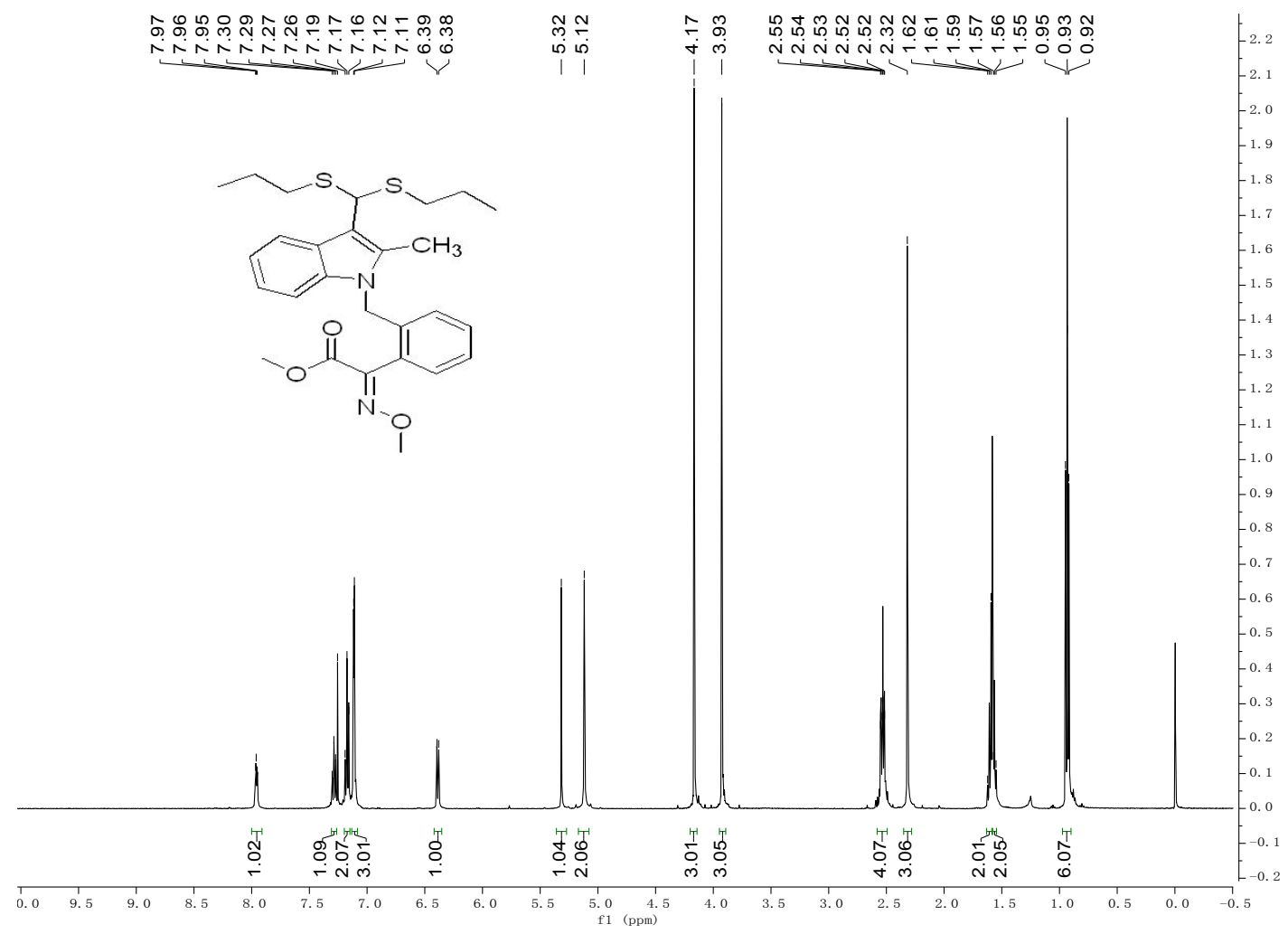

Figure 81. ${ }^{1} \mathrm{H}$ NMR ((400 MHz, DMSO- $\left.d_{6}\right)$ spectrum of compound D24. 


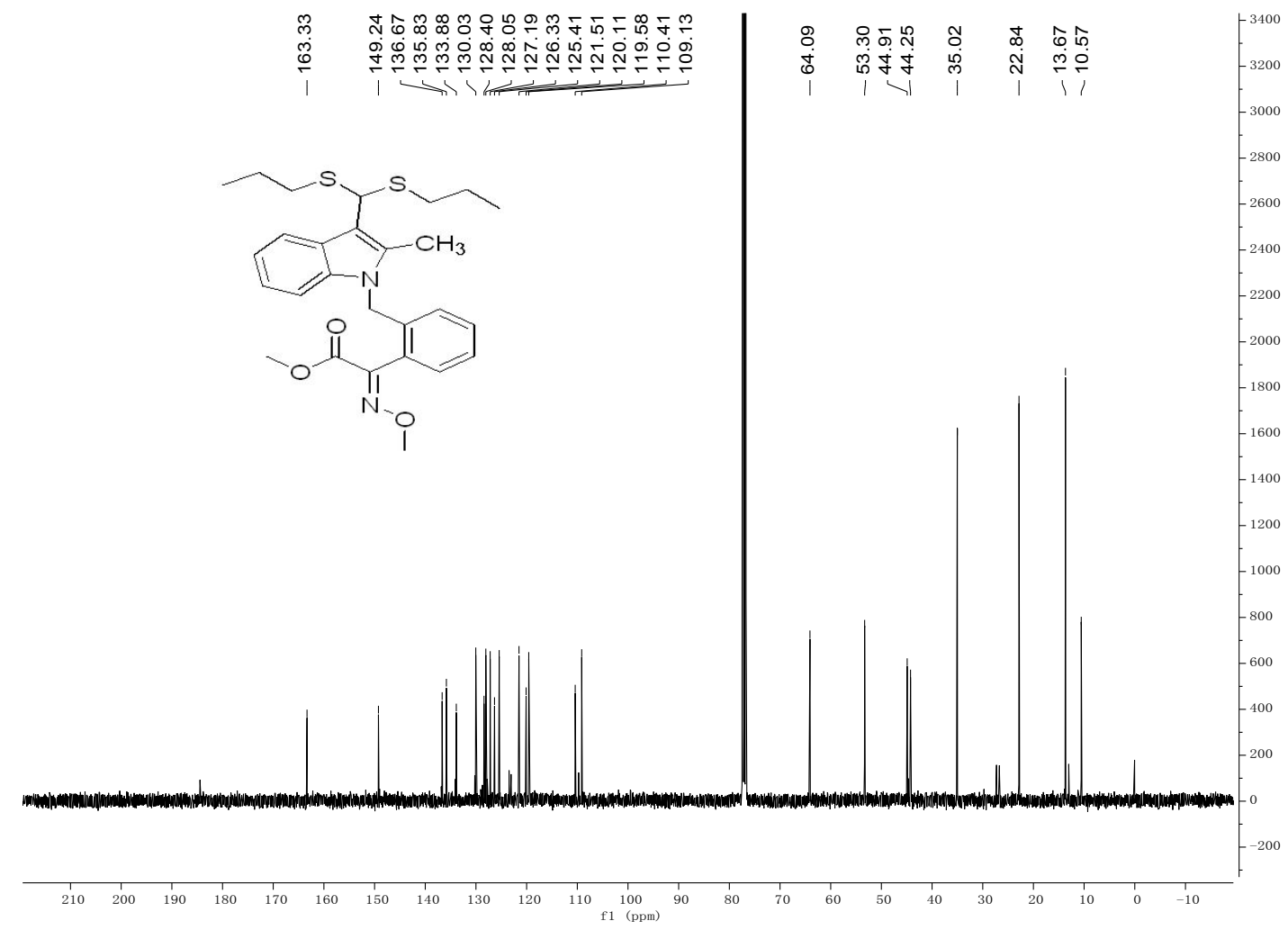

Figure 82. ${ }^{13} \mathrm{C}$ NMR $\left(100 \mathrm{MHz}, \mathrm{DMSO}-d_{6}\right)$ spectrum of compound D24.

2019041722 \#81 Rा: 0.78 AV: 1 N: 3.73E8 T: FIMS+ pESI Ful ms [100.0000-1000.0000]

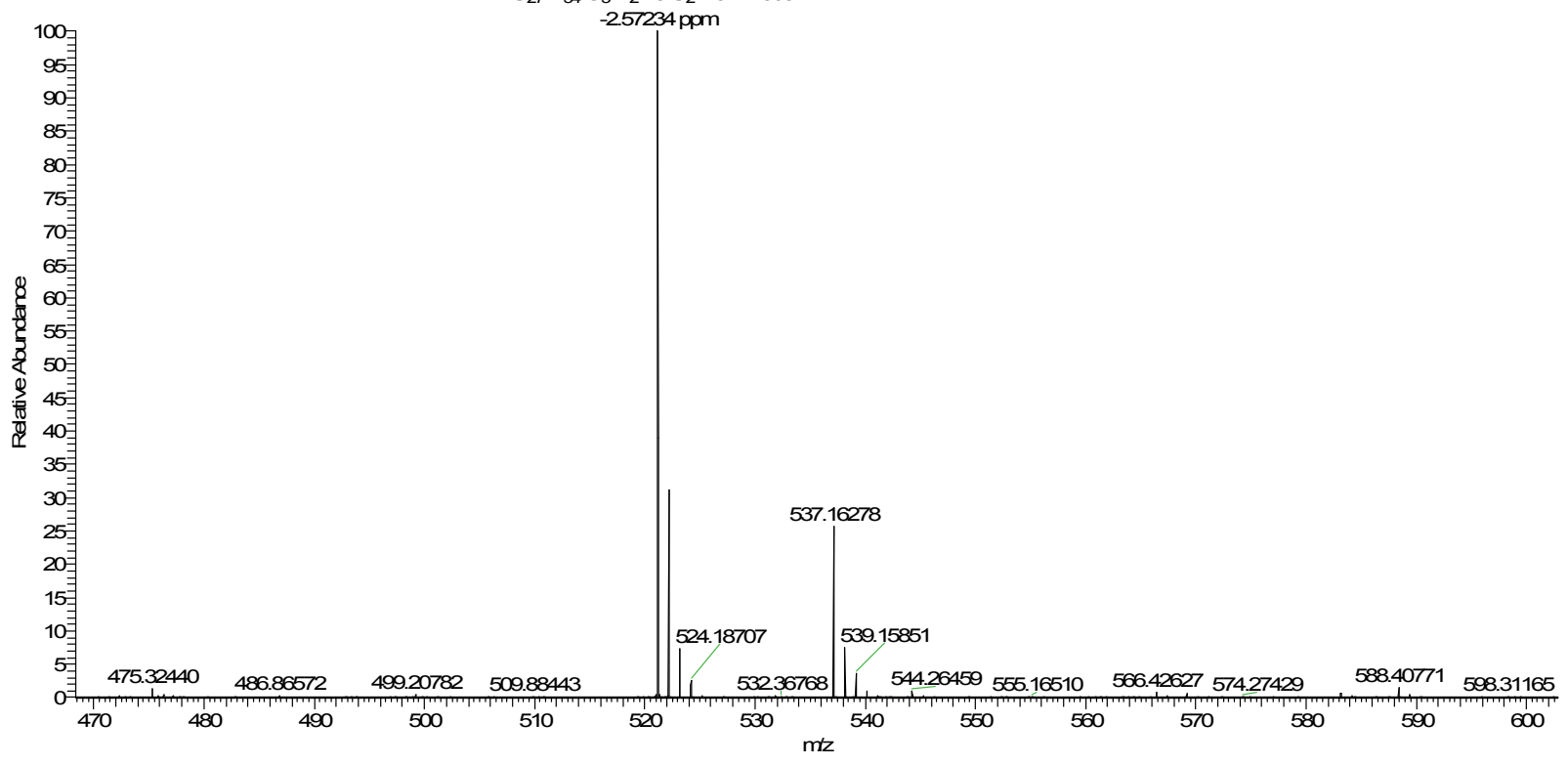

Figure 83. HRMS of compound D24. 


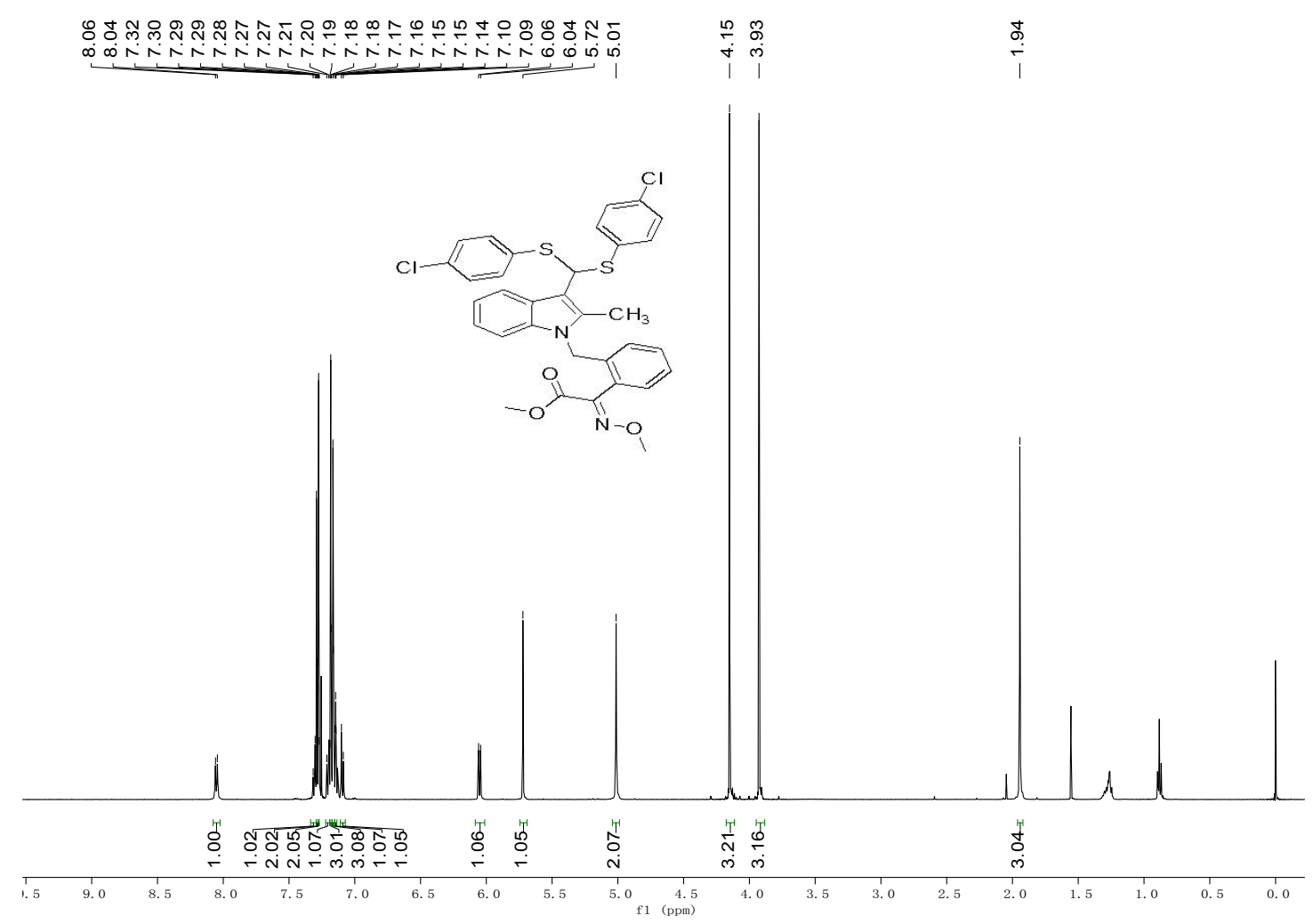

Figure 84. ${ }^{1} \mathrm{H}$ NMR $\left(\left(400 \mathrm{MHz}, \mathrm{DMSO}-d_{6}\right)\right.$ spectrum of compound D25.

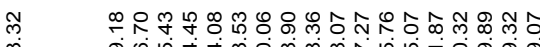

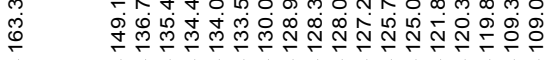

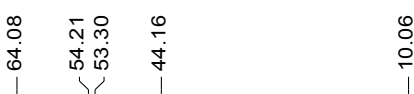

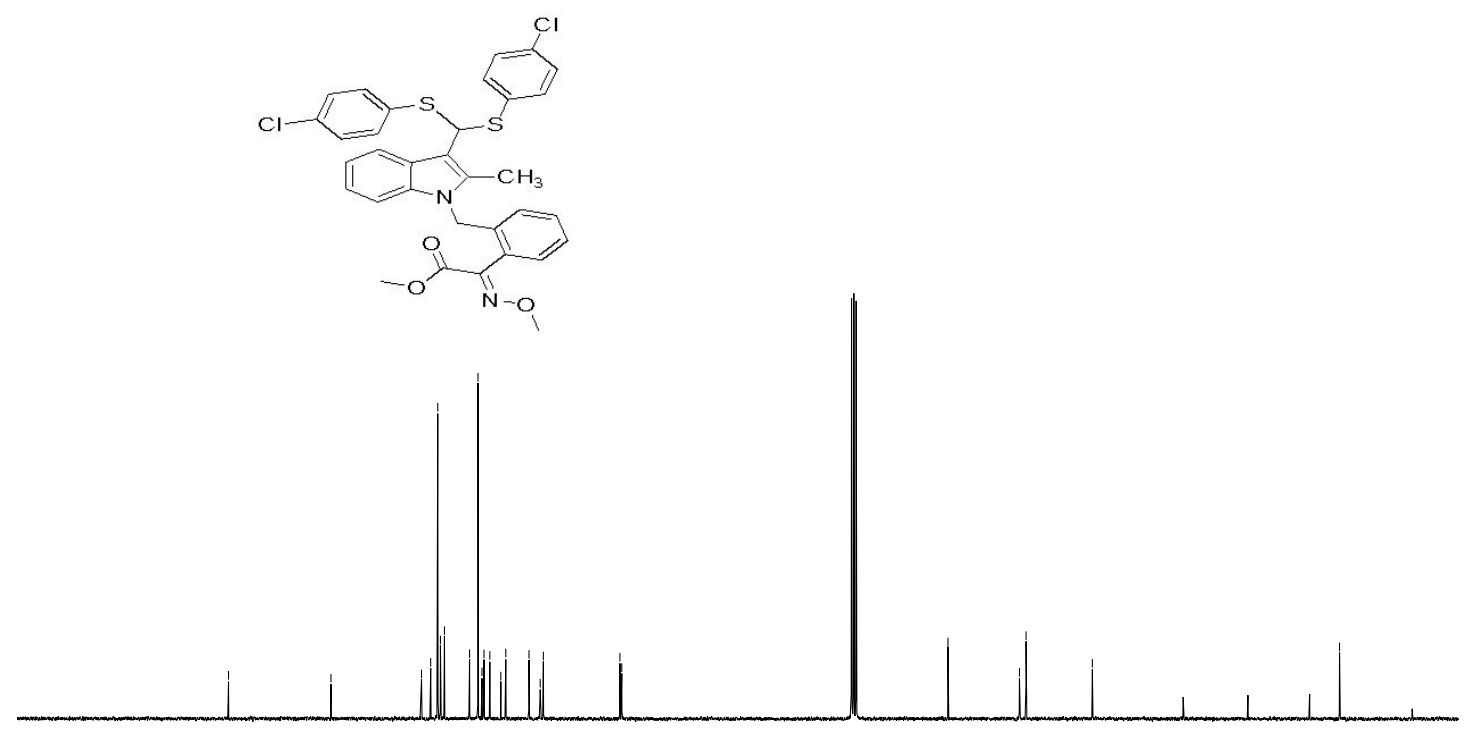

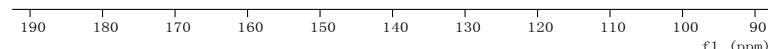

Figure 85. ${ }^{13} \mathrm{C}$ NMR $\left(100 \mathrm{MHz}, \mathrm{DMSO}-d_{6}\right)$ spectrum of compound D25. 
201904172ß\#115 Rा: 1.10 AV: 1 N: 4.70E7

T: FIMS + pESI Full ms [100.0000-1000.0000]

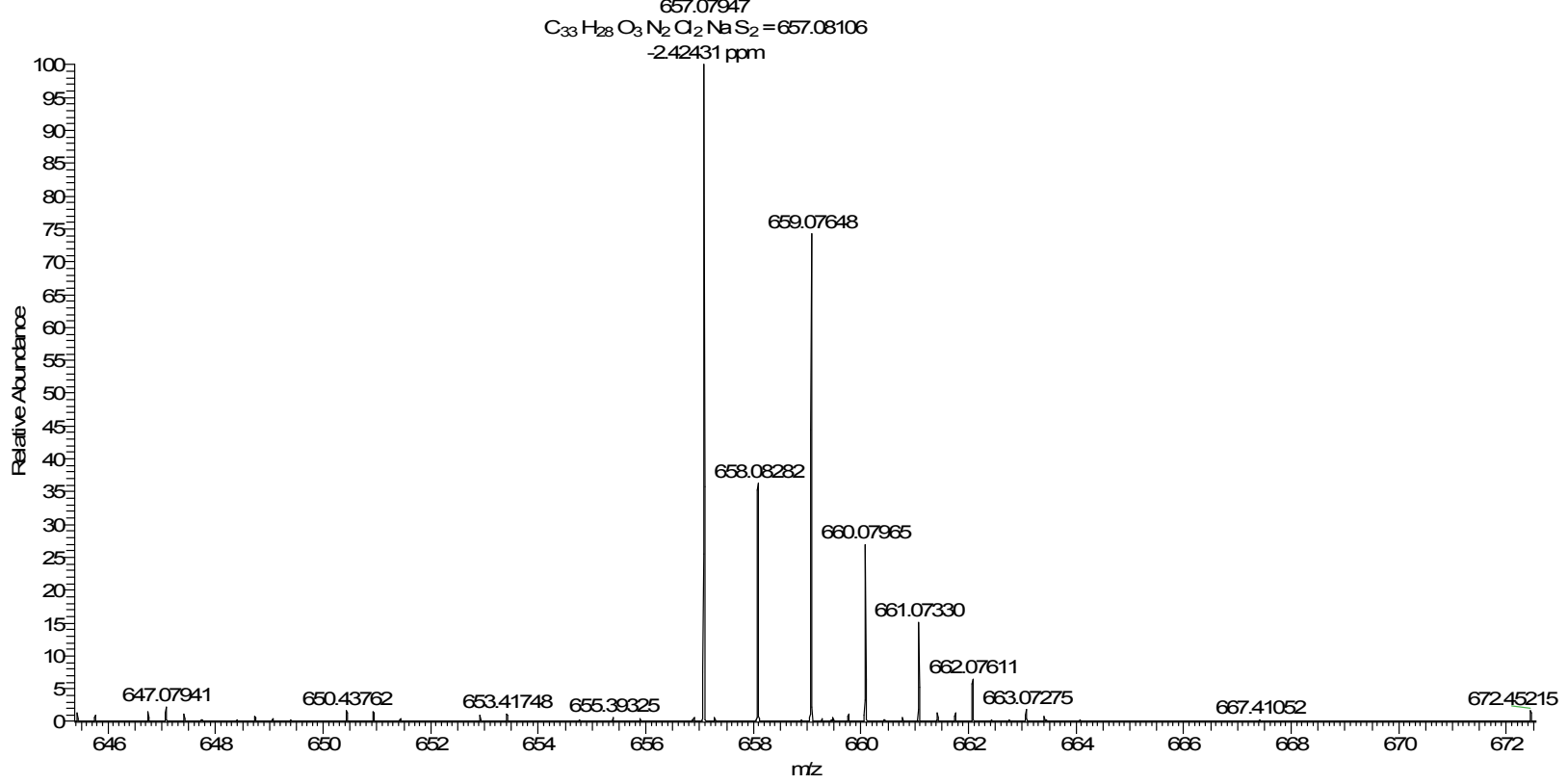

Figure 86. HRMS of compound D25.

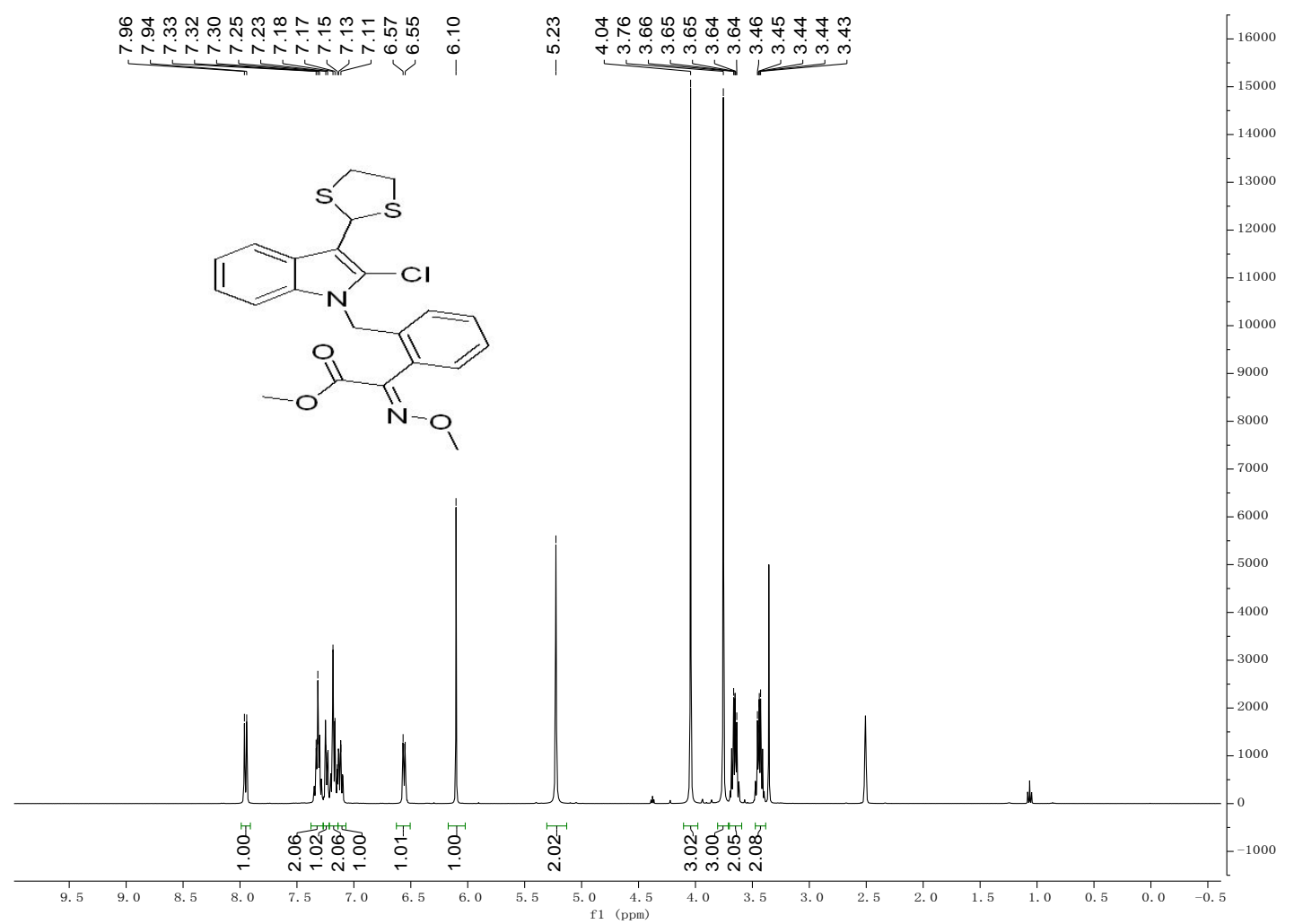

Figure 87. ${ }^{1} \mathrm{H}$ NMR ((400 MHz, DMSO- $\left.d_{6}\right)$ spectrum of compound D26. 


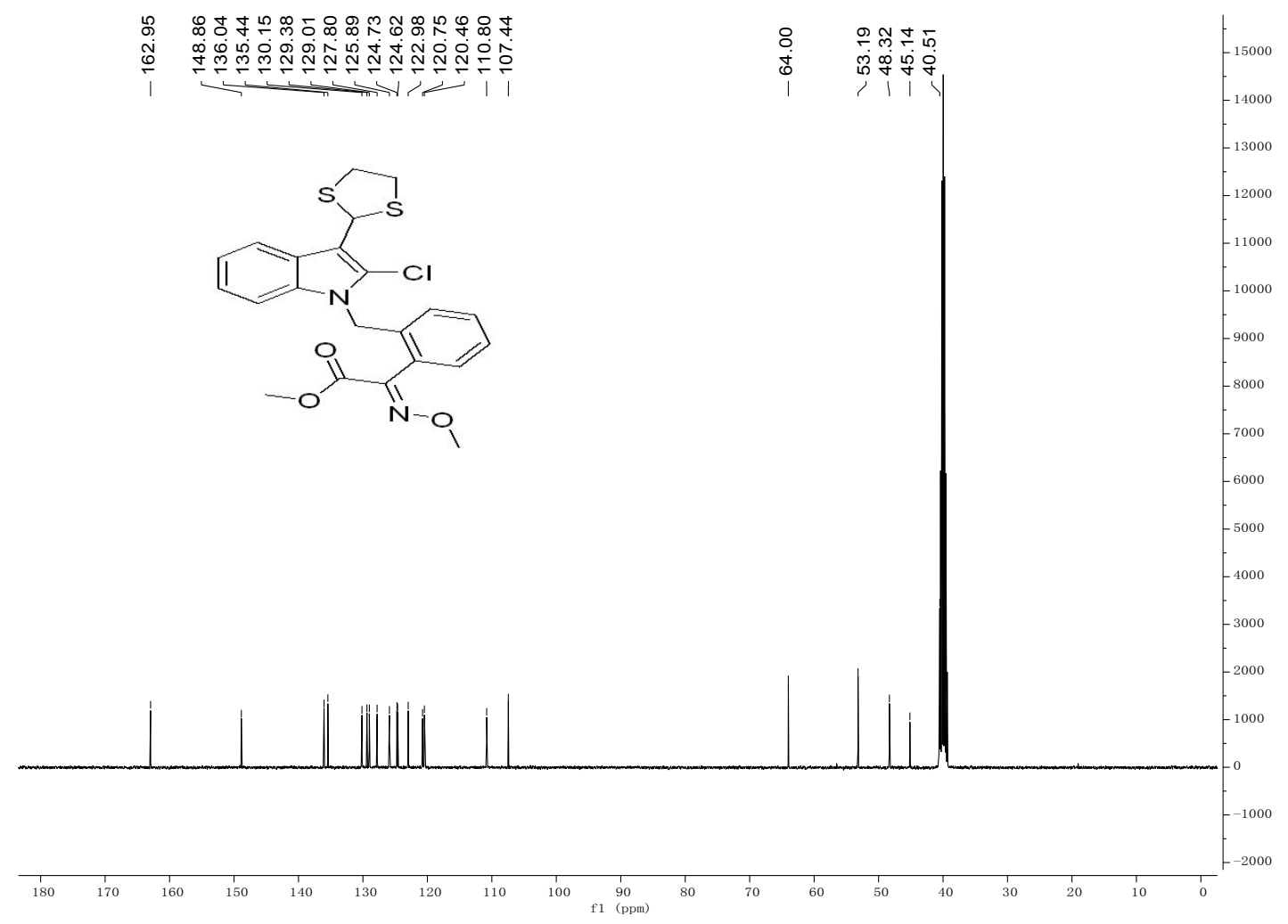

Figure 88. ${ }^{13} \mathrm{C}$ NMR $\left(100 \mathrm{MHz}\right.$, DMSO- $\left.d_{6}\right)$ spectrum of compound D26.

2019041252 \#59 Rा: 0.57 AV: 1 N. 5.18E T: FIMS + pESI Full ms [100.0000-1000.0000]

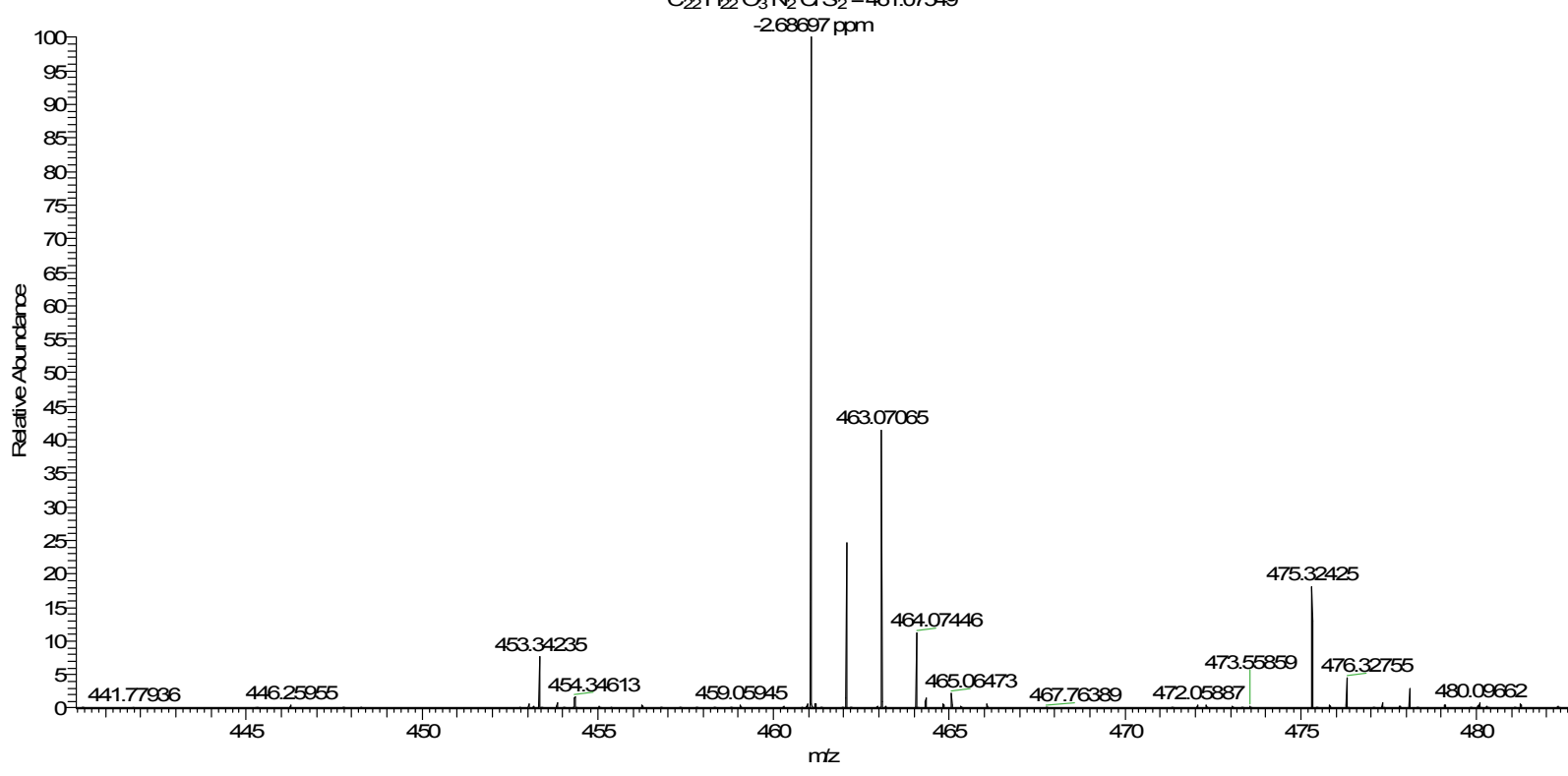

Figure 89. HRMS of compound D26. 


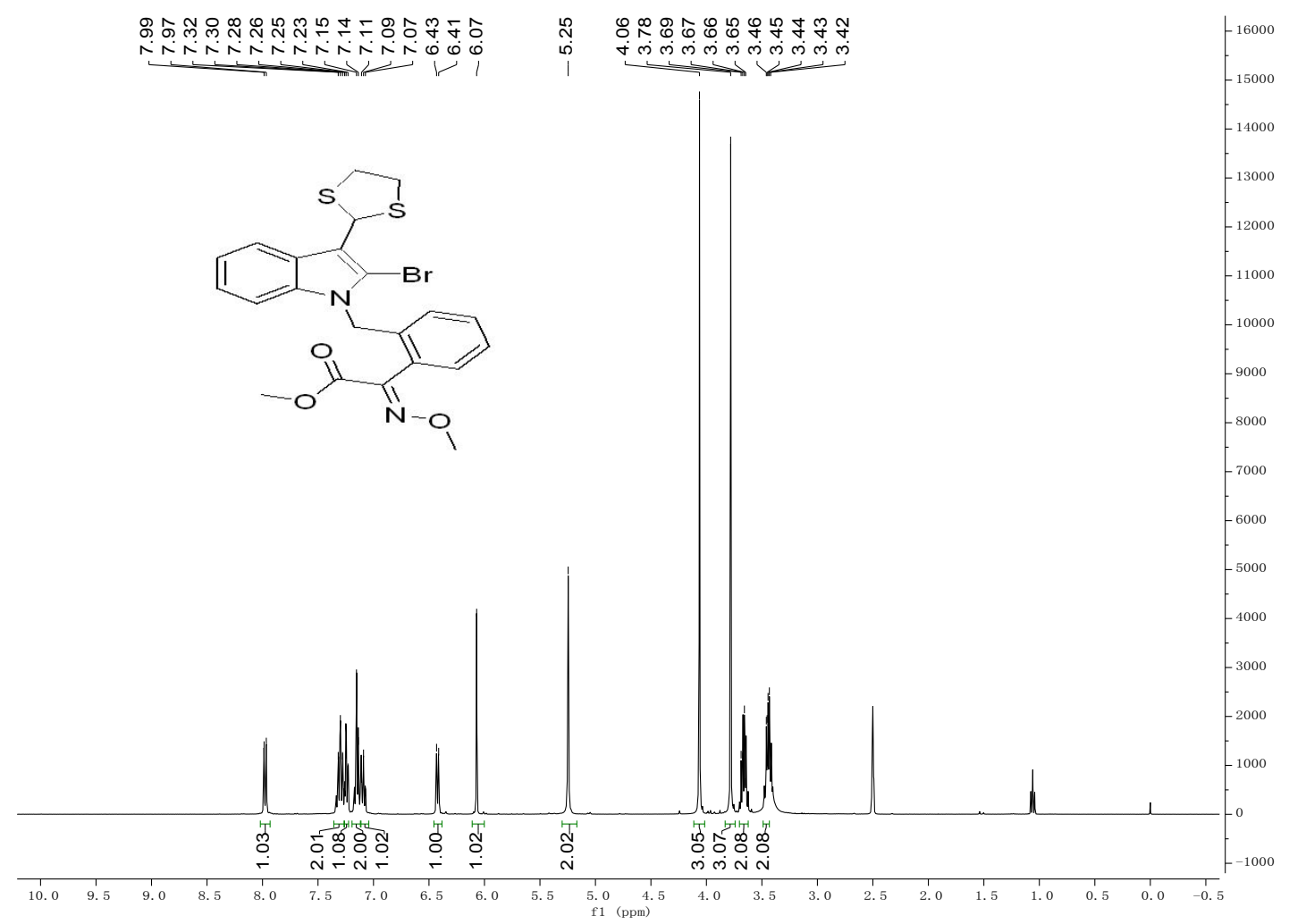

Figure 90. ${ }^{1} \mathrm{H}$ NMR ((400 MHz, DMSO- $\left.d_{6}\right)$ spectrum of compound D27.

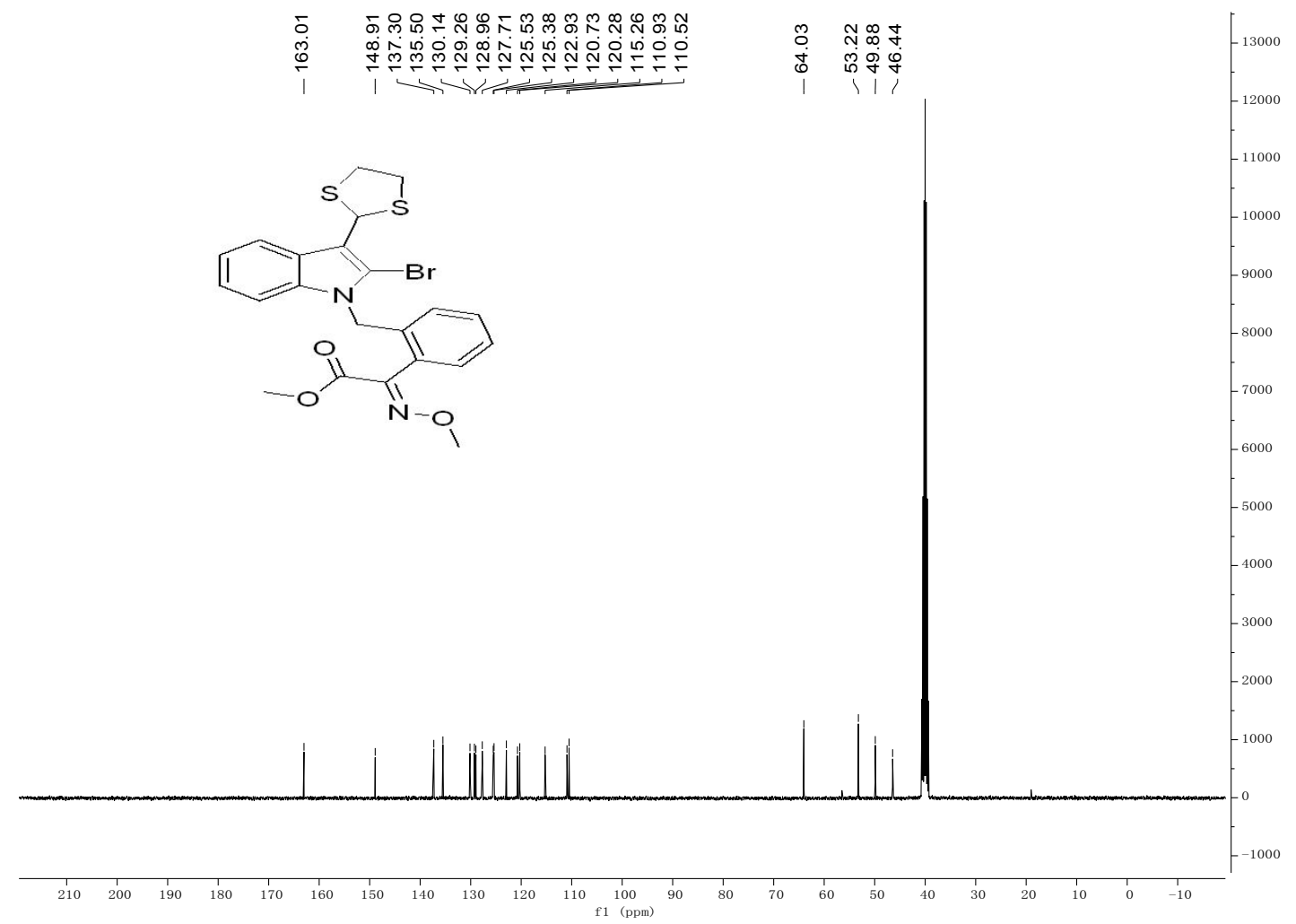

Figure 91. ${ }^{13} \mathrm{C}$ NMR (100 MHz, DMSO- $d_{6}$ ) spectrum of compound D27. 


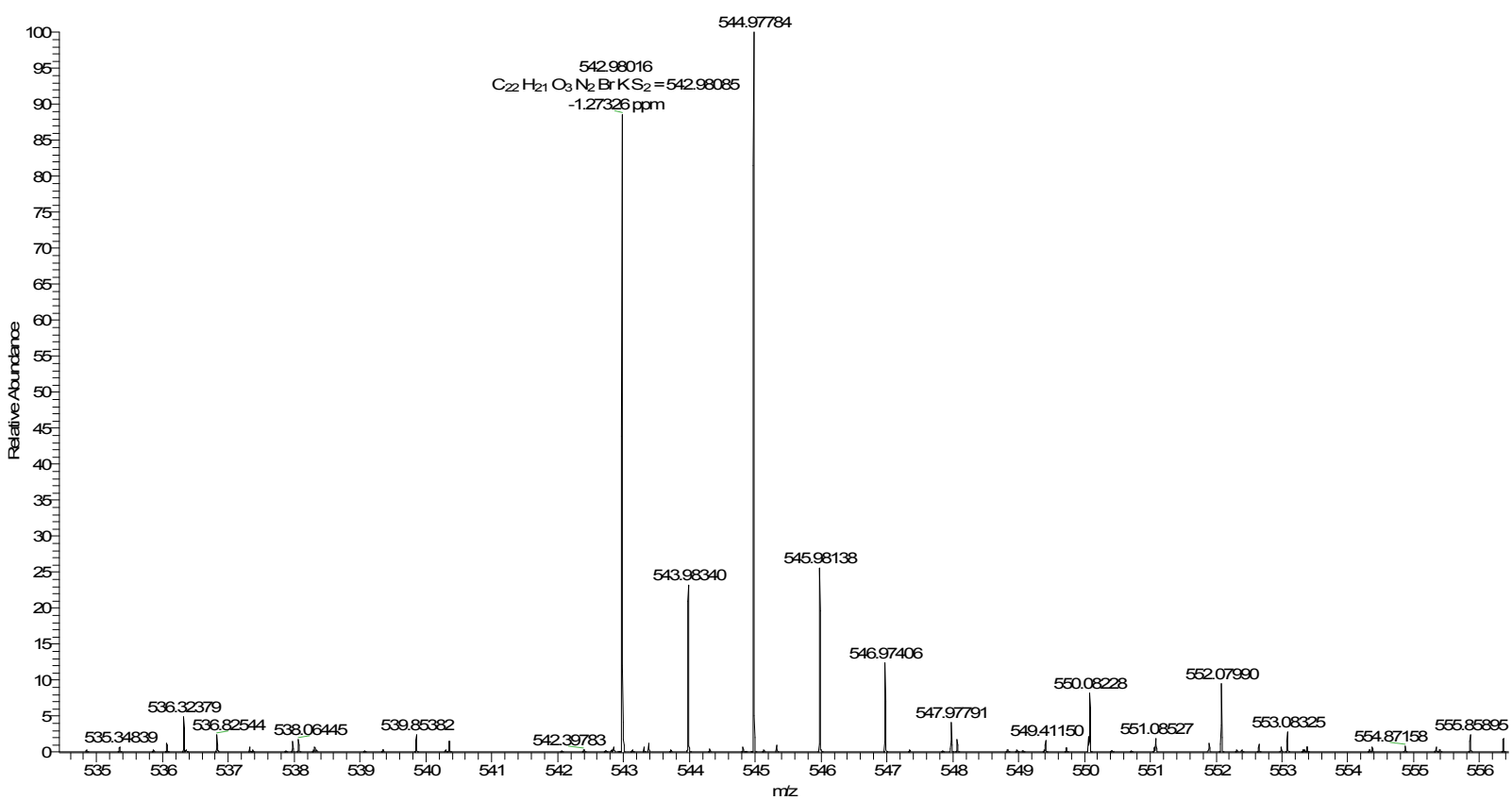

Figure 92. HRMS of compound D27.

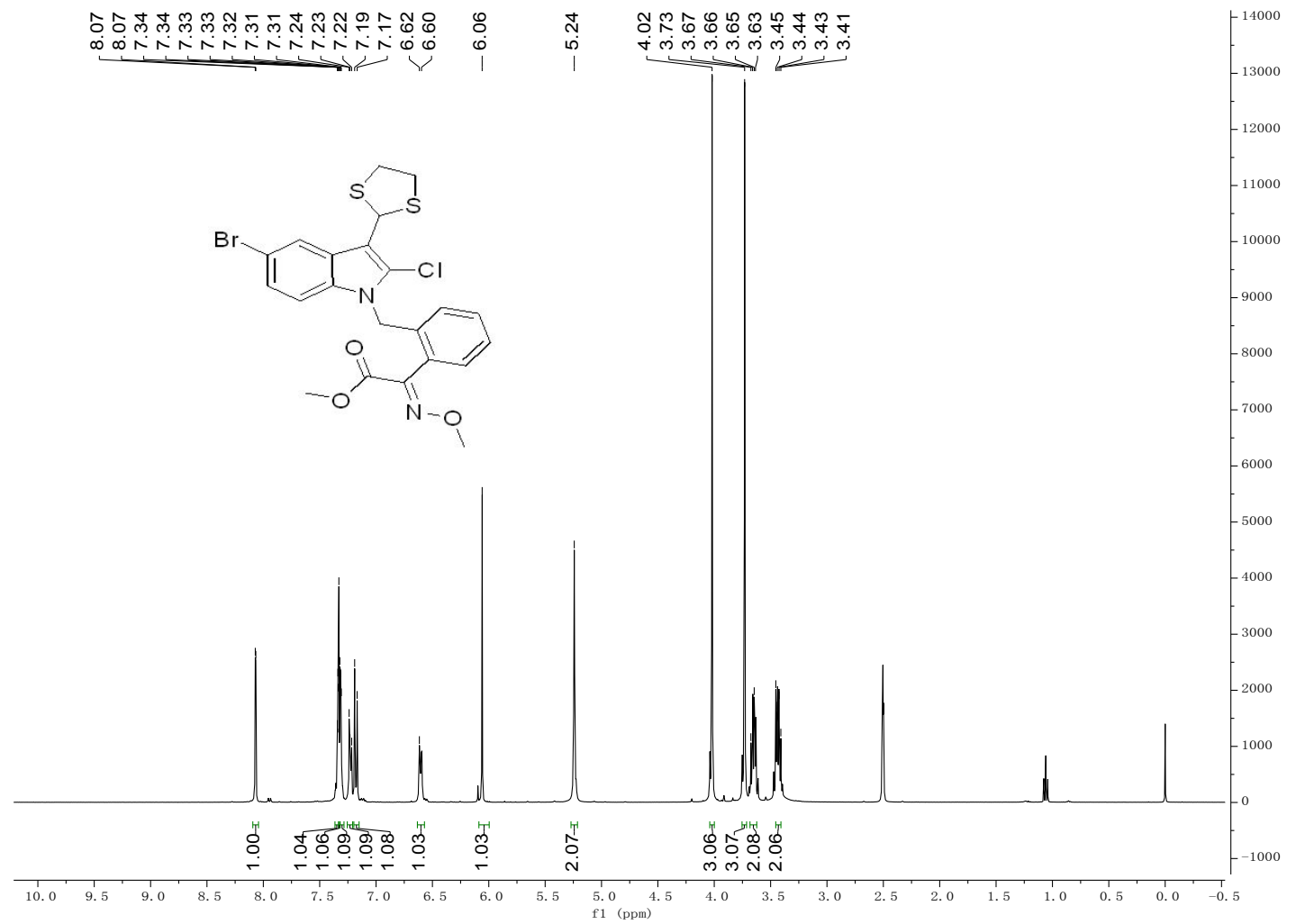

Figure 93. ${ }^{1} \mathrm{H}$ NMR ((400 MHz, DMSO- $\left.d_{6}\right)$ spectrum of compound D28. 


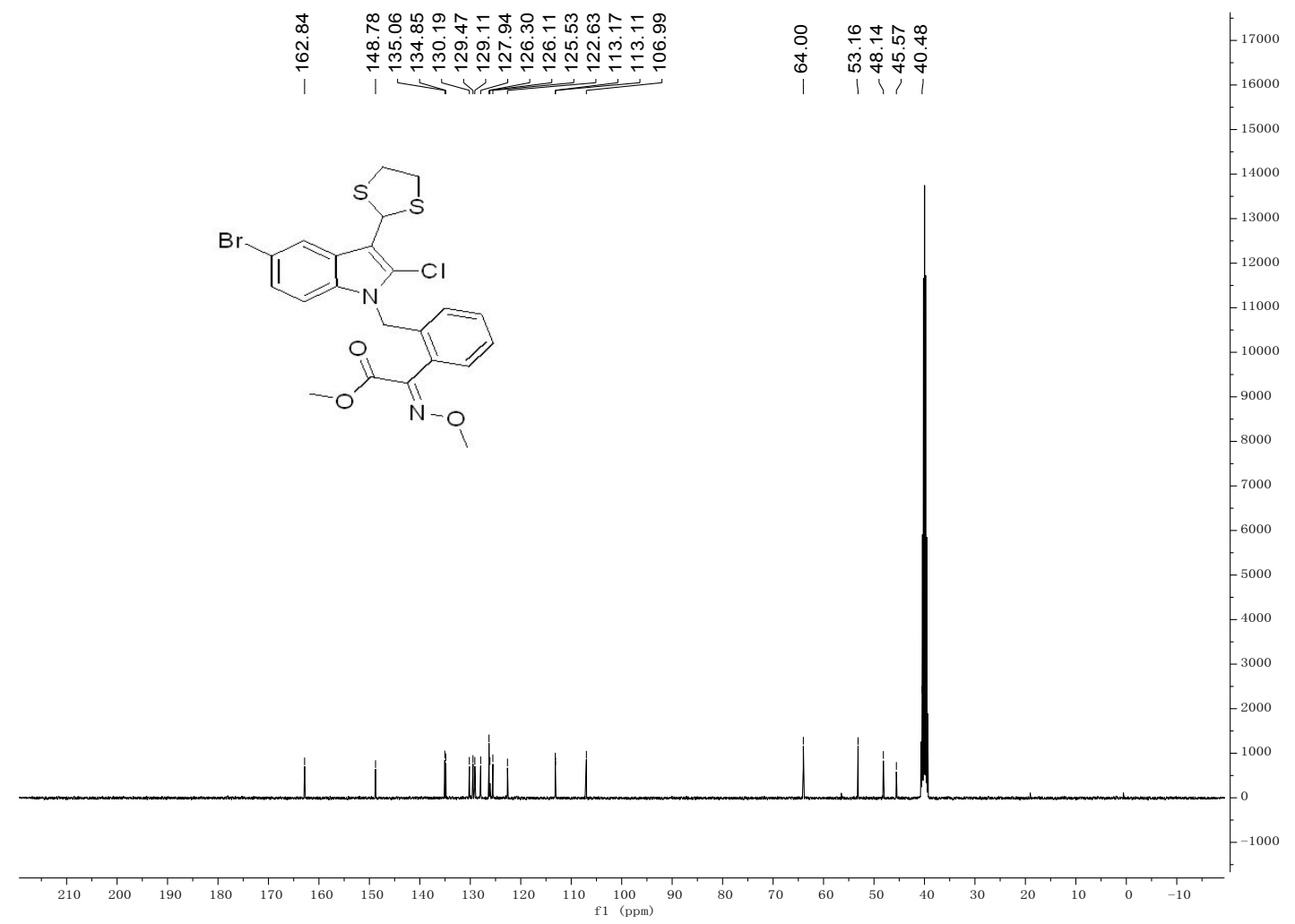

Figure 94. ${ }^{13} \mathrm{C}$ NMR (100 MHz, DMSO- $d_{6}$ ) spectrum of compound D28.

2019041724\#71 Rा: 0.68 AV: 1 N. 7.06El T: FIMS + pESI Full ms [100.0000-1000.0000]

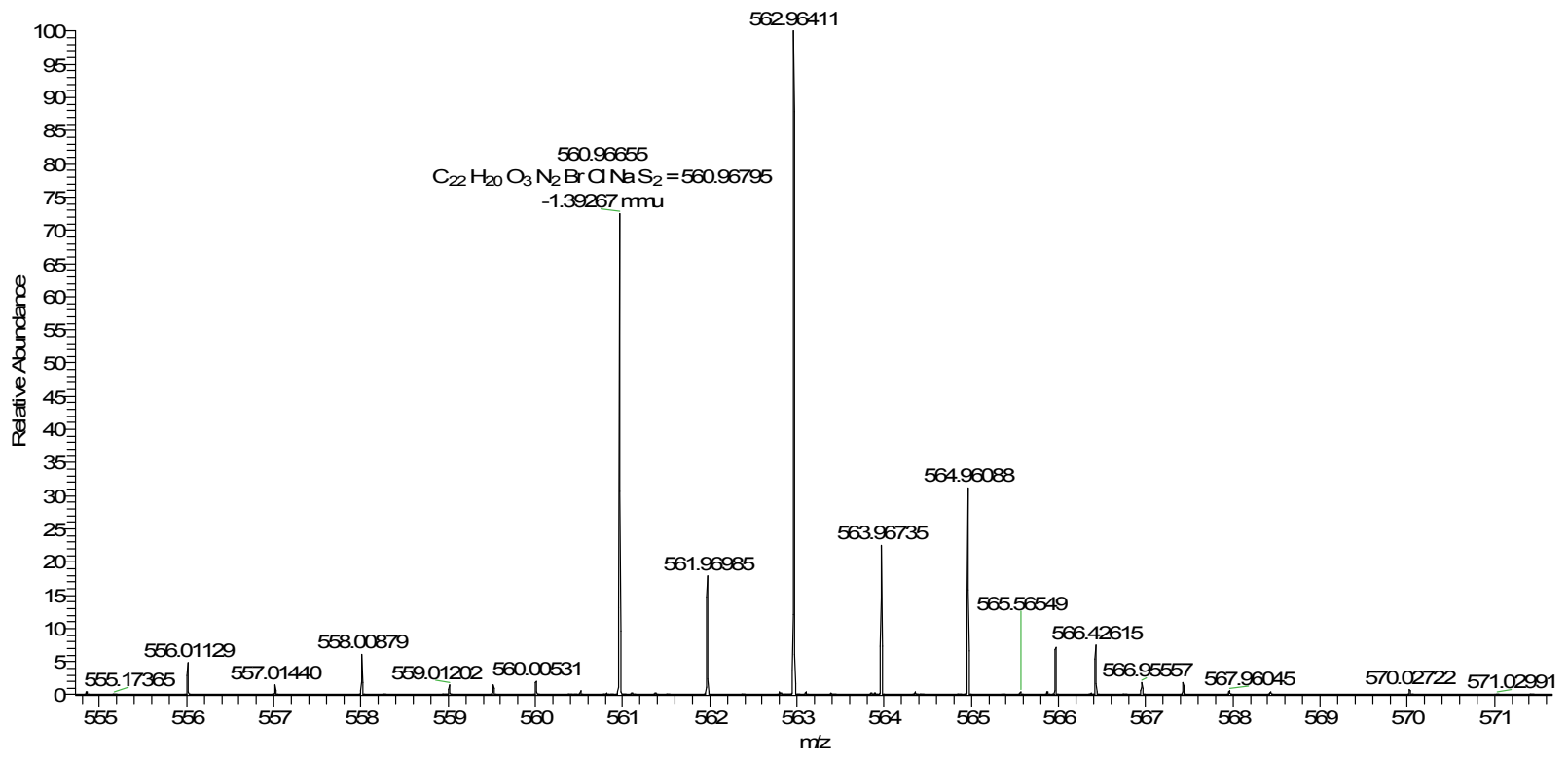

Figure 95. HRMS of compound D28. 